\title{
Classification of Flavonoid Metabolomes via Data Mining and Quantification of Hydroxyl NMR Signals
}

Yang Yu, ${ }^{\dagger}$ Guido F. Pauli, ${ }^{\dagger \dagger}$ Lingyi Huang, ${ }^{\dagger}$ Li-She Gan,${ }^{\S}$ Richard B. van Breemen, ${ }^{\dagger}$ Dianpeng Li, ${ }^{\|}$James B. McAlpine, ${ }^{\dagger}$ David C. Lankin, ${ }^{\dagger}$ and Shao-Nong Chen ${ }^{\dagger, \grave{\ddagger}, *}$

${ }^{\dagger}$ UIC Center for Botanical Dietary Supplements Research, PCRPS and Department of Pharmaceutical Sciences, and ${ }^{\ddagger}$ Institute for Tuberculosis Research, College of Pharmacy, University of Illinois at Chicago, Chicago, Illinois 60612, United States ${ }^{\S}$ College of Pharmaceutical Sciences, Zhejiang University, Hangzhou, 310058, China

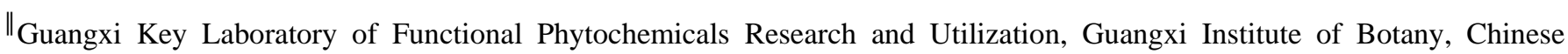
Academy of Sciences, Guilin 541006, China

- SUPPORTING INFORMATION 
Section S1. Details of Experimental Procedures \& Structure Elucidation 6

Section S1A. General Experimental Procedures $\quad 6$

Section S1B. Building NMR Metabolomic Tools $\quad 6$

Section S1C. Samples and Sample Preparation $\quad 6$

$\begin{array}{ll}\text { Section S1D. Fractionation by HPLC } & 7\end{array}$

$\begin{array}{ll}\text { Section S1E. LC-MS Conditions } & 7\end{array}$

Section S2. Structure Elucidation and Verification of 5-OH Flavonoids 9

Section S3. Structure Verification of 5-OH Flavonoids 15

Figure S1. Original structure of licoisoflavanone B reported in the literature (A) vs its revised 15 structure determined as relicoisoflavanone B (B)

Figure S2. Original structure of licoisoflavanone C reported in the literature (A) vs its revised 15 structure determined as relicoisoflavanone $\mathrm{C}(\mathrm{B})$

Figure S3. ${ }^{1} \mathrm{H}-{ }^{1} \mathrm{H}$ COSY $(-)$ and selected HMBC $(\mathrm{H} \rightarrow \mathrm{C})$ correlations of the original structure of licoisoflavanone $\mathrm{B}$ reported in the literature (A) vs its revised structure determined as relicoisoflavanone $\mathrm{B}(\mathrm{B})$

Figure S4. ${ }^{1} \mathrm{H}-{ }^{1} \mathrm{H}$ COSY $(-)$ and selected HMBC $(\mathrm{H} \rightarrow \mathrm{C})$ correlations of the original structure of licoisoflavanone $\mathrm{C}$ reported in the literature $(\mathrm{A})$ vs its revised structure determined as relicoisoflavanone $\mathrm{C}(\mathrm{B})$

Section S4. Structure Elucidation and Verification Related Tables

Table S1. Comparison of the ${ }^{1} \mathrm{H}$ and ${ }^{13} \mathrm{C}$ NMR spectroscopic data of licoisoflavanone B vs relicoisoflavanone $\mathrm{B}$, compound 1 , and licoisoflavanone $\mathrm{C}$ vs relicoisoflavanone $\mathrm{C}$

Table S2. The HMBC patterns of licoisoflavanone B vs relicoisoflavanone B, compound 1, and licoisoflavanone $\mathrm{C}$ vs relicoisoflavanone $\mathrm{C}$

Table S3. The HMBC patterns mined from literature data of compounds isolated from licorice species (GU, GG, and GI)

Table S4. The metabolomic HMBC patterns from experimental data of GU/GG/GI MeOH 22 fractions

Table S5. The HMBC patterns of 15 compounds identified in GU-MF-11, GU-MF-12, and 23 GU-MF-14-20

Section S5. References Related to Structure Elucidation and Verification 24

Section S6. Further Supporting Figures \& Tables 28

Figure S5. Comparison of the ${ }^{1} \mathrm{H}$ NMR spectra of WF, 50\% MF, MF, and AF for GU 28

Figure S6. Comparison of the ${ }^{1} \mathrm{H}$ NMR spectra of GU-MF, GG-MF, and GI-MF 29

Figure S7. The chemical shifts of the 5-/2'-OH hydrogens and the three HMBC correlated 30 carbons of the following flavonoid skeletons: flavonone, isoflavonone, flavononol, flavone, isoflavone, flavonol, its 3-O-methyl derivative, its 3-O-glycoside, dihydrochalcone, chalcone, and $\alpha$-hydroxyl dihydrochalcone

Figure S8. The preparative HPLC profile of GU-MF at $254 \mathrm{~nm}$, divided into 20 subfractions, 31 
GU-MF-1 to GU-MF-20

Figure S9. The 5-/2'-OH hydrogen chemical shifts and structures of the three model 32 compounds, Y1, Y2, and Y3

Table S6. Bond lengths, second-order stabilization energies $\Delta E^{(2)}$, and natural charges of the 33 hydrogen atoms of the $\mathrm{C}(4 / 1)=\mathrm{O} \ldots \mathrm{H}-\mathrm{O}-\mathrm{C}\left(5 / 2^{\prime}\right)$ hydrogen bonds in the model compounds $\mathrm{Y} 1$, $\mathrm{Y} 2$, and $\mathrm{Y} 3$

Figure S10. B3LYP/6-31+G(d,p) optimized lowest energy 3D conformers of the model 34 compounds, $\mathrm{Y} 1, \mathrm{Y} 2$, and $\mathrm{Y} 3$

Table S7. Energy analysis of the conformers of the model compounds, Y1, Y2, and Y3

Table S8. NBO analysis data for conformers of the model compounds, Y1, Y2, and Y3

Table S9. Second-order stabilization energies $\Delta E^{(2)}(\mathrm{kcal} / \mathrm{mol})$ of the hydrogen bonds of the 36 conformers of the model compounds, Y1, Y2, and Y3

Table S10. The relative percentage of the eight subtypes in GU, GG, and GI, respectively 36

Figure S11. The ${ }^{1} \mathrm{H}$ NMR spectrum of GU-MF

Figure S12. The HMBC spectrum of GU-MF

Figure S13. The ${ }^{1} \mathrm{H}$ NMR spectrum of GG-MF

Figure S14. The HMBC spectrum of GG-MF

Figure S15. The ${ }^{1} \mathrm{H}$ NMR spectrum of GI-MF

Figure S16. The HMBC spectrum of GI-MF

Figure S17. The ${ }^{1} \mathrm{H}$ NMR spectrum of GU-MF-11

Figure S18. The ${ }^{1} \mathrm{H}$ NMR spectrum of compound 13 in GU-MF-11 44

Figure S19. The ${ }^{1} \mathrm{H}$ NMR spectrum of compound 14 in GU-MF-11 45

Figure S20. The ${ }^{1} \mathrm{H}$ NMR spectrum of compound 11 in GU-MF-11 46

Figure S21. The ${ }^{1} \mathrm{H}$ NMR spectrum of compound 3 in GU-MF-11 47

Figure S22. The ${ }^{1} \mathrm{H}-{ }^{1} \mathrm{H}$ COSY spectrum of GU-MF-11 48

Figure S23. The HSQC spectrum of GU-MF-11 49

Figure S24. The HMBC spectrum of GU-MF-11 50

Figure S25. The IT-TOF TIC and mass chromatogram of GU-MF-11 51

Figure S26. The (+)-HRESIMS and (-)-HRESIMS spectra of one of compounds 3, 11, and 1352 in GU-MF-11

Figure S27. The (+)-HRESIMS and (-)-HRESIMS spectra of one of compounds 3, 11, and 1353 in GU-MF-11

Figure S28. The (+)-HRESIMS and (-)-HRESIMS spectra of one of compounds 3, 11, and 1354 in GU-MF-11

Figure S29. The (+)-HRESIMS and (-)-HRESIMS spectra of compound 14 in GU-MF-11 55

Figure S30. The ${ }^{1} \mathrm{H}$ NMR spectrum of GU-MF-12 56

Figure S31. The ${ }^{1} \mathrm{H}$ NMR spectrum of compound 8 in GU-MF-12 57

Figure S32. The ${ }^{1} \mathrm{H}$ NMR spectrum of compound 10 in GU-MF-12 58

Figure S33. The ${ }^{1} \mathrm{H}-{ }^{1} \mathrm{H}$ COSY spectrum of GU-MF-12

$\begin{array}{ll}\text { Figure S34. The HSQC spectrum of GU-MF-12 } & 60\end{array}$

Figure S35. The HMBC spectrum of GU-MF-12 61 
Figure S36. The IT-TOF TIC and mass chromatogram of GU-MF-12

Figure S37. The (+)-HRESIMS and (-)-HRESIMS spectra of one of compounds 8 and 10 in 63 GU-MF-12

Figure S38. The (+)-HRESIMS and (-)-HRESIMS spectra of one of the compounds 8 and 10 in 64 GU-MF-12

Figure S39. The ${ }^{1} \mathrm{H}$ NMR spectrum of GU-MF-14 65

Figure S40. The ${ }^{1} \mathrm{H}$ NMR spectrum of compound 12 in GU-MF-14 66

Figure $\mathrm{S} 41$. The ${ }^{1} \mathrm{H}-{ }^{1} \mathrm{H}$ COSY spectrum of GU-MF-14

Figure S42. The HSQC spectrum of GU-MF-14

Figure S43. The HMBC spectrum of GU-MF-14

Figure S44. The IT-TOF TIC and mass chromatogram of GU-MF-14

Figure S45. The (+)-HRESIMS and (-)-HRESIMS spectra of compound 12 in GU-MF-14 71

Figure S46. The ${ }^{1} \mathrm{H}$ NMR spectrum of GU-MF-15

Figure S47. The ${ }^{1} \mathrm{H}$ NMR spectrum of compound 15 in GU-MF-15

Figure S48. The ${ }^{1} \mathrm{H}$ NMR spectrum of compound 2 in GU-MF-15

Figure S49. The ${ }^{1} \mathrm{H}-{ }^{1} \mathrm{H}$ COSY spectrum of GU-MF-15

Figure S50. The HSQC spectrum of GU-MF-15

Figure S51. The HMBC spectrum of GU-MF-15

Figure S52. The IT-TOF TIC and mass chromatogram of GU-MF-15

Figure S53. The (+)-HRESIMS and (-)-HRESIMS spectra of compound 15 in GU-MF-15 79

Figure S54. The (+)-HRESIMS and (-)-HRESIMS spectra of compound 2 in GU-MF-15 80

Figure S55. The ${ }^{1} \mathrm{H}$ NMR spectrum of GU-MF-16

Figure S56. The ${ }^{1} \mathrm{H}$ NMR spectrum of compound 5 in GU-MF-16

Figure S57. The ${ }^{1} \mathrm{H}-{ }^{1} \mathrm{H}$ COSY spectrum of GU-MF-16

Figure S58. The HSQC spectrum of GU-MF-16

Figure S59. The HMBC spectrum of GU-MF-16

Figure S60. The IT-TOF TIC and mass chromatogram of GU-MF-16

Figure S61. The (+)-HRESIMS and (-)-HRESIMS spectra of compound 5 in GU-MF-16 87

Figure S62. The ${ }^{1} \mathrm{H}$ NMR spectrum of GU-MF-17 88

Figure S63. The ${ }^{1} \mathrm{H}$ NMR spectrum of compound 6 in GU-MF-17 89

Figure S64. The ${ }^{1} \mathrm{H}-{ }^{1} \mathrm{H}$ COSY spectrum of GU-MF-17 90

Figure S65. The HSQC spectrum of GU-MF-17 91

Figure S66. The HMBC spectrum of GU-MF-17 92

Figure S67. The IT-TOF TIC and mass chromatogram of GU-MF-17 93

Figure S68. The (+)-HRESIMS and (-)-HRESIMS spectra of compound 6 in GU-MF-17 94

Figure S69. The ${ }^{1} \mathrm{H}$ NMR spectrum of GU-MF-18 95

Figure S70. The ${ }^{1} \mathrm{H}$ NMR spectrum of compound 1 in GU-MF-18 96

Figure S71. The ${ }^{1} \mathrm{H}-{ }^{1} \mathrm{H}$ COSY spectrum of GU-MF-18 97

Figure S72. The HSQC spectrum of GU-MF-18 98

Figure S73. The HMBC spectrum of GU-MF-18 99

Figure S74. The IT-TOF TIC and mass chromatogram of GU-MF-18 100 
Figure S75. The (+)-HRESIMS and (-)-HRESIMS spectra of compound 1 in GU-MF-18

Figure S76. The ${ }^{1} \mathrm{H}$ NMR spectrum of GU-MF-18-1

Figure S77. The ${ }^{1} \mathrm{H}$ NMR spectrum of compound 1 in GU-MF-18-1

Figure S78. The ${ }^{1} \mathrm{H}-{ }^{1} \mathrm{H}$ COSY spectrum of GU-MF-18-1

Figure S79. The HSQC spectrum of GU-MF-18-1

Figure S80. The HMBC spectrum of GU-MF-18-1

Figure S81. Expansion of the HMBC spectrum of GU-MF-18-1

Figure S82. The IT-TOF TIC and mass chromatogram of GU-MF-18-1

Figure S83. The (+)-HRESIMS and (-)-HRESIMS spectra of compound 1 in GU-MF-18-1 109

Figure S84. The ${ }^{1} \mathrm{H}$ NMR spectrum of GU-MF-19

Figure S85. The ${ }^{1} \mathrm{H}$ NMR spectrum of compound 9 in GU-MF-19

Figure S86. The ${ }^{1} \mathrm{H}-{ }^{1} \mathrm{H}$ COSY spectrum of GU-MF-19

Figure S87. The HSQC spectrum of GU-MF-19

Figure S88. The HMBC spectrum of GU-MF-19

Figure S89. The IT-TOF TIC and mass chromatogram of GU-MF-19

Figure S90. The (+)-HRESIMS and (-)-HRESIMS spectra of compound 9 in GU-MF-19

Figure S91. The ${ }^{1} \mathrm{H}$ NMR spectrum of GU-MF-20

Figure S92. The ${ }^{1} \mathrm{H}$ NMR spectrum of compound 7 in GU-MF-20

Figure S93. The ${ }^{1} \mathrm{H}$ NMR spectrum of compound 4 in GU-MF-20

Figure S94. The ${ }^{1} \mathrm{H}-{ }^{1} \mathrm{H}$ COSY spectrum of GU-MF-20

Figure S95. The HSQC spectrum of GU-MF-20

Figure S96. The HMBC spectrum of GU-MF-20

Figure S97. The IT-TOF TIC and mass chromatogram of GU-MF-20

Figure S98. The (+)-HRESIMS and (-)-HRESIMS spectra of compound 7 in GU-MF-20

Figure S99. The (+)-HRESIMS and (-)-HRESIMS spectra of compound 4 in GU-MF-20

Section S7. Flowchart of Literature Mining 
The HPLC purification was using a Shimadzu Prominence HPLC system with LC-20AB pump and SPD-20A UV/VIS detector (Kyoto, Japan) on GROM-SIL 120 ODS-4 HE column $(300 \times 20$ mm, $5 \mu \mathrm{m})$ from Watrex International (San Francisco, CA, USA). HRESIMS data were obtained on a Shimadzu LC-IT-TOF mass spectrometer. All the three species of licorice were extracted with an Accelerated Solvent Extraction (ASE 350) from Dionex Corporation (Sunnyvale, CA, USA). All collected HPLC fractions were dried using a Genevac miVac centrifugal vacuum concentrator (Ipswich, England). Freeze-drying was performed on a Labconco Freezone 4.5 (Kansas City, MO, USA). ${ }^{1} \mathrm{H}$ NMR and HMBC spectra of GU-MF, GG-MF, and GI-MF, as well as ${ }^{1} \mathrm{H}$ NMR, ${ }^{1} \mathrm{H}-{ }^{1} \mathrm{H}$ COSY, HSQC, and HMBC (hmbcetgpnd) spectra of GU-MF-18-1 were performed on a Bruker (Rheinstetten, Germany) Avance $600 \mathrm{MHz}$ NMR spectrometer equipped with a $5 \mathrm{~mm}$ TXI cryoprobe, whereas ${ }^{1} \mathrm{H}$ NMR, ${ }^{1} \mathrm{H}-{ }^{1} \mathrm{H}$ COSY, HSQC, and HMBC (hmbcgplpndqf) spectra of GU-MF-1-GU-MF-20 were acquired on a Bruker Avance III DRX-500 spectrometer equipped with a $1.7 \mathrm{~mm}$ room temperature microprobe. ${ }^{1} \mathrm{H} /{ }^{13} \mathrm{C}$ NMR chemical shifts were referenced to the residual dimethyl sulfoxide- $d_{6}$ (DMSO- $d_{6}$ ) solvent signals $\delta 2.500 / 39.510$ in the corresponding ${ }^{1} \mathrm{H} /{ }^{13} \mathrm{C}$ NMR spectra, respectively. All the ${ }^{13} \mathrm{C}$ NMR data were retrieved from either HSQC or HMBC spectra. Offline 1D and 2D NMR data processing was performed with Mnova NMR software package (v.6.0.2, MestReLab Research S.L., A Coruña, Spain) using default parameters, or with NUTS Pro (v. 201004, Acorn NMR Inc., Livermore, CA, USA) using a Lorentz-Gaussian window function (line broadening $=-0.3 \mathrm{~Hz}$, Gaussian factor $=0.05$ ). Three times zero fillings were applied prior to Fourier transformation, and the resulting NMR spectra were subjected to manual phase adjustment and automatic baseline correction using polynomial functions.

\section{SECTION S1B. BUILDING NMR METABOLOMIC TOOLS.}

The NMR metabolomic tools were built on the basis of our previous studies and developed methodologies, as described in references ${ }^{1-9}$.

\section{SECTION SIC. SAMPLES AND SAMPLE PREPARATION.}

10 gram of each DNA verified licorice raw material was extracted with EtOH: $i$-PrOH: $\mathrm{H}_{2} \mathrm{O}(90: 5: 5)$, using the ASE 350 setup at $80{ }^{\circ} \mathrm{C}$, for $30 \mathrm{~min}$ of static time at $1500 \mathrm{psi}$, for a total of $45 \mathrm{~min}$ of extraction. 
The extractions were performed for three times to yield three licorice extracts (GU, GG, and GI). Each extract was fractionated on XAD-2 (Sigma-Aldrich, St Louis, MO, USA) column with $\mathrm{H}_{2} \mathrm{O}$ (WF), 50\% $\mathrm{MeOH}(50 \% \mathrm{MF}), \mathrm{MeOH}(\mathrm{MF})$, and Acetone (AF), respectively. The 5-OH flavonoids/flavanoids/2'-OH chalcones were enriched into methanol fractions, GU-MF, GG-MF, and GI-MF. To validate the method, GU-MF was further fractionated with HPLC on ODS semi-prep column to yield twenty subfractions, GU-MF-1-GU-MF-20. To make the NMR spectra of GU-MF-18 less complicated for elucidation and validation of structure 1, GU-MF-18 was further separated over a Sephadex LH-20 column to get two fractions, and compound 1 was concentrated in GU-MF-18-1.

NMR samples of GU-MF, GG-MF, and GI-MF were prepared at the following weights: GU-MF, 25.1 mg; GG-MF, $25.1 \mathrm{mg}$; GI-MF, $25.1 \mathrm{mg}$, followed by the addition of $600 \mu \mathrm{L}$ of $99.9 \%$ DMSO- $d_{6}$ (Cambridge Isotope Laboratory (CIL), Inc., Andover, MA, USA). Next, the solutions were transferred to 5 mm NMR tubes. NMR sample of GU-MF-18-1 was prepared at the following weight: $1.82 \mathrm{mg}$, followed by the addition of $175 \mu \mathrm{L}$ of $99.9 \%$ DMSO- $d_{6}$. Meanwhile, GU-MF-1-GU-MF-20 were prepared at the following weights: GU-MF-1, $1.27 \mathrm{mg}$; GU-MF-2, $1.13 \mathrm{mg}$; GU-MF-3, $3.20 \mathrm{mg}$; GU-MF-4, $0.46 \mathrm{mg}$; GU-MF-5, 1.31 mg; GU-MF-6, 0.66 mg; GU-MF-7, 1.37 mg; GU-MF-8, 0.85 mg; GU-MF-9, 1.42 mg; GU-MF-10, 2.34 mg; GU-MF-11, 2.35 mg; GU-MF-12, 1.71 mg; -13, 1.07 mg; -14, 2.36 mg; -15, 3.32 mg; $-16,2.48 \mathrm{mg} ;-17,1.05 \mathrm{mg} ;-18,2.43 \mathrm{mg} ;-19,1.70 \mathrm{mg} ;-20,1.53 \mathrm{mg}$, followed by the addition of $45 \mu \mathrm{L}$ of "100\%" DMSO- $d_{6}(\mathrm{CIL})$. Next, the $35 \mu \mathrm{L}$ solutions were transferred to $1.7 \mathrm{~mm}$ NMR tubes.

\section{SECTION SID. FRACTIONATION BY HPLC.}

The elution of GU-MF in HPLC was achieved with a gradient of acetonitrile (A) and aqueous $0.1 \%$ acetic acid (B) starting with 35\% A from 0 to 2.5 min, reaching 35-70\% A over 2.5-35 min, keeping 70-76\% A over 35-75 min, getting 76-80\% A over 75-90 min, achieving 80-90\% A over 90-100 min, reaching 90-100\% A over 100-105 min, and keeping 100\% A over 105-115 min, with a flow rate of $0.8 \mathrm{~mL} / \mathrm{min} .20$ subfractions were acquired from above HPLC fractionation (Figure S8, Supporting information).

\section{SECTION SIE. LC-MS CONDITIONS.}

Each sample of GU-MF-1-GU-MF-20 was analyzed using high resolution accurate mass measurement with data-dependent product ion MS/MS on a Shimadzu LCMS-ITTOF hybrid mass spectrometer equipped with a Shimadzu Prominence UFLC-XR HPLC system. Separations were obtained using a Waters (Milford, 
MA, USA) XTerra C18, $1.0 \times 150 \mathrm{~mm}, 3.5 \mu \mathrm{m}$ HPLC column. The mobile phase consisted of a $18 \mathrm{~min}$ gradient from water containing $0.1 \%$ formic acid to acetonitrile. The flow rate was $0.2 \mathrm{~mL} / \mathrm{min}$, and the column temperature was $35^{\circ} \mathrm{C}$.

In the electrospray source, the nitrogen nebulizing gas flow was $1.5 \mathrm{~L} / \mathrm{min}$, the heating gas flow was 10 $\mathrm{L} / \mathrm{min}$, the interface temperature $200{ }^{\circ} \mathrm{C}$, the $\mathrm{CDL}$ temperature $200{ }^{\circ} \mathrm{C}$, and the heat block $200{ }^{\circ} \mathrm{C}$. Positive and negative ion electrospray mass spectra were recorded from $\mathrm{m} / \mathrm{z} 100$ to 1000 . For positive and negative scans, product ion mass spectra were recorded in $0.375 \mathrm{~s}$ using unit resolution selection in the ion trap and a resolving power about 10,000 in the time-of-flight (TOF) sector. The detector voltage was $1.65 \mathrm{kV}$.

The predicted formulas were calculated based on the Software in Shimadzu IT-TOF instrument: Accurate Mass Calculator. The ppm values for each constituent were included.

The elution of GU-MF-11 in HPLC was achieved with a gradient of acetonitrile (A) and aqueous $0.1 \%$ formic acid (B) starting with 35\% A from 0 to $3.0 \mathrm{~min}$, reaching 35-55\% A over 3.0-14.0 min, getting 55-100\% A over 14.0-15.5 min, keeping 100\% A over 15.5-18.0 min, with a flow rate of $0.2 \mathrm{~mL} / \mathrm{min}$.

The elution of GU-MF-12 in HPLC was achieved with a gradient of acetonitrile (A) and aqueous $0.1 \%$ formic acid (B) starting with $20 \%$ A from 0 to $0.5 \mathrm{~min}$, reaching 20-30\% A over 0.5-3.0 min, getting 30-50\% A over 3.0-10.0 min, achieving 50-100\% A over 10.0-15.5 min, keeping 100\% A over 15.5-18.0 min, with a flow rate of $0.2 \mathrm{~mL} / \mathrm{min}$.

The elution of GU-MF-14-GU-MF-16 in HPLC were achieved with a gradient of acetonitrile (A) and aqueous $0.1 \%$ formic acid (B) starting with $25 \%$ A from 0 to $0.5 \mathrm{~min}$, reaching $25-35 \%$ A over 0.5-3.0 min, getting 35-55\% A over 3.0-10.0 min, achieving 55-100\% A over 10.0-15.5 min, keeping 100\% A over 15.5-18.0 min, with a flow rate of $0.2 \mathrm{~mL} / \mathrm{min}$.

The elution of GU-MF-17-GU-MF-20 in HPLC were achieved with a gradient of acetonitrile (A) and aqueous $0.1 \%$ formic acid (B) starting with $30 \%$ A from 0 to $0.5 \mathrm{~min}$, reaching $30-40 \%$ A over 0.5-3.0 min, getting 40-60\% A over 3.0-10.0 min, achieving 60-100\% A over 10.0-15.5 min, keeping 100\% A over 15.5-18.0 min, with a flow rate of $0.2 \mathrm{~mL} / \mathrm{min}$. 


\section{SECTION S2. STRUCTURE ELUCIDATION AND VERIFICATION OF 5-OH FLAVONOIDS.}

According to the developed methodology, two compounds (10 and 8) were identified in GU-MF-12, which belonged to two major 5-OH flavonoids. The NMR data $\left(\delta_{\mathrm{H}} 12.90,1 \mathrm{H}, \mathrm{s}, 5-\mathrm{OH} ; \delta_{\mathrm{C}} 159.6, \mathrm{C}-5\right.$; 98.5, C-6; 104.5, C-10; 13.22, 1H, s, 5-OH; $\delta_{\mathrm{C}} 158.8$, C-5; 111.1, C-6; 104.5, C-10) acquired from the ${ }^{1} \mathrm{H}$ NMR and HMBC spectra of GU-MF-12 (Figures S30-32 and S35, Supporting Information), established their structural types: one subtype II-1, 5,7-dihydroxy-8-prenyl (iso)flavone for 10, and one subtype II-3, 5,7-dihydroxy-6-prenyl (iso)flavone for $\mathbf{8}$. The structures of the two compounds (10 and $\mathbf{8}$ ) were deduced together. The HSQC spectrum (Figure S34, Supporting Information) of GU-MF-12 revealed the presence of two proton signals $\left(\delta_{\mathrm{H}} 8.38,1 \mathrm{H}, \mathrm{s}, \mathrm{H}-2 ; \delta_{\mathrm{C}} 153.9, \mathrm{C}-2 ; \delta_{\mathrm{H}} 8.28,1 \mathrm{H}, \mathrm{s}, \mathrm{H}-2 ; \delta_{\mathrm{C}} 153.9, \mathrm{C}-2\right)$ having the same peak areas as those of proton resonances $12.90 \mathrm{ppm}$ in $\mathbf{1 0}$ and $13.22 \mathrm{ppm}$ in $\mathbf{8}$, respectively, indicating that both of $\mathbf{1 0}$ and $\mathbf{8}$ are isoflavones. The integral values of H-2 in $\mathbf{1 0}$ and $\mathbf{8}$ indicated that the quantities of two compounds are almost equal. The NMR data $\left(\delta_{\mathrm{H} / \mathrm{C}} 7.38 / 130.2,2 \mathrm{H}, \mathrm{d}, J=8.2 \mathrm{~Hz}, \mathrm{CH}-2^{\prime}, 6^{\prime}\right.$; $6.81 / 115.1,2 \mathrm{H}, \mathrm{d}, J=8.2 \mathrm{~Hz}, \mathrm{CH}-3^{\prime}, 5^{\prime} ;$ and $\delta_{\mathrm{H} / \mathrm{C}} 7.36 / 130.2,2 \mathrm{H}, \mathrm{d}, J=8.2 \mathrm{~Hz}, \mathrm{CH}-2^{\prime}, 6^{\prime} ; 6.82 / 115.1,2 \mathrm{H}, \mathrm{d}$, $J=8.2 \mathrm{~Hz}, \mathrm{CH}-3^{\prime}, 5^{\prime}$ ) showed that both of compounds $\mathbf{1 0}$ and $\mathbf{8}$ had 4'-hydroxy substituted B-rings, which was proved by the COSY correlations (Figure S33, Supporting Information) of $\mathrm{H}-2^{\prime} / \mathrm{H}-3^{\prime}$ and H-5'/H-6' as well as the HMBC correlations (Figure S35, Supporting Information) from H-2', H-3', H-5', and H-6' to C-4' $\left(\delta_{\mathrm{C}} 157.4\right)$ for both of $\mathbf{1 0}$ and 8. Considering the (-)-HRESIMS (Figures S37 and S38, Supporting Information) at $m / z 337.1035[\mathrm{M}-\mathrm{H}]^{-}$(calcd for $\mathrm{C}_{20} \mathrm{H}_{17} \mathrm{O}_{5}{ }^{-}, 337.1081$ ) and $\mathrm{m} / \mathrm{z} 337.1044[\mathrm{M}-\mathrm{H}]^{-}(\mathrm{calcd}$

for $\mathrm{C}_{20} \mathrm{H}_{17} \mathrm{O}_{5}{ }^{-}, 337.1081$ ) for $\mathbf{1 0}$ and $\mathbf{8}$, or vice versa, their structures were determined as lupiwighteone ${ }^{10}$ and wighteone, ${ }^{10}$ respectively.

Compound 12 in GU-MF-14, a major 5-OH flavonoid, was identified as subtype II-2, 5,7-dihydroxy (iso)flavonone $\left(\delta_{\mathrm{H}} 12.85,1 \mathrm{H}, \mathrm{s}, 5-\mathrm{OH} ; \delta_{\mathrm{C}} 161.8, \mathrm{C}-5 ; 98.9, \mathrm{C}-6 ; 104.6, \mathrm{C}-10\right)$ on the basis of the ${ }^{1} \mathrm{H}$ NMR data and HMBC spectrum (Figures S39, S40, and S43, Supporting Information). The ${ }^{1} \mathrm{H}$ NMR data and HSQC spectrum (Figures S39, S40, and S42, Supporting Information) of GU-MF-14 revealed the presence of one proton signal $\left(\delta_{\mathrm{H}} 8.17,1 \mathrm{H}, \mathrm{s}, \mathrm{H}-2 ; \delta_{\mathrm{C}} 155.5, \mathrm{C}-2\right)$ having the same peak area as that of proton resonance $12.85 \mathrm{ppm}$ in 12, indicating 12 was an isoflavone. Based on the analysis of the ${ }^{1} \mathrm{H}-{ }^{1} \mathrm{H}$ COSY spectrum (Figure S41, Supporting Information), two AB-coupled aromatic hydrogens $\left(\delta_{\mathrm{H}} 6.33,1 \mathrm{H}, \mathrm{d}, J=\right.$ $\left.8.2 \mathrm{~Hz}, \mathrm{H}-5^{\prime} ; 6.88,1 \mathrm{H}, \mathrm{d}, J=8.2 \mathrm{~Hz}, \mathrm{H}-6^{\prime} ; \delta_{\mathrm{C}} 107.3, \mathrm{C}-5^{\prime} ; 131.2, \mathrm{C}-6^{\prime}\right)$ and a $1^{\prime}, 2^{\prime}, 3^{\prime}, 4^{\prime}-$ tetrasubstituted B-ring were determined in 12, which was proved by the HMBC correlations (Figure S43, Supporting 
Information) from $\mathrm{H}^{-5} 5^{\prime}$ to $\mathrm{C}-1^{\prime}\left(\delta_{\mathrm{C}} 111.2\right), \mathrm{C}-3^{\prime}\left(\delta_{\mathrm{C}} 109.8\right)$, and C-4' $\left(\delta_{\mathrm{C}} 153.7\right)$; from H-6' to C-3 $\left(\delta_{\mathrm{C}} 120.6\right)$, C-2' $\left(\delta_{\mathrm{C}} 151.0\right)$, and C-4'. The NMR data $\left(\delta_{\mathrm{H}} 6.67,1 \mathrm{H}, \mathrm{d}, J=10.0 \mathrm{~Hz}, \mathrm{H}-1^{\prime \prime} ; 5.68,1 \mathrm{H}, \mathrm{d}, J=10.0 \mathrm{~Hz}, \mathrm{H}-2^{\prime \prime}\right.$; $\left.\delta_{\mathrm{C}} 116.8, \mathrm{C}-1^{\prime \prime} ; 128.7, \mathrm{C}-2^{\prime \prime}\right)$ revealed that 12 possessed a 2,2-dimethylpyran ring with the same pattern as that of licoisoflavone $\mathrm{B},{ }^{10}$ which was proved by the HMBC correlations (Figure S43, Supporting Information) from $\mathrm{H}-1^{\prime \prime}$ to $\mathrm{C}-2^{\prime}, \mathrm{C}-3^{\prime}, \mathrm{C}-4^{\prime}$, and $\mathrm{C}-3^{\prime \prime}\left(\delta_{\mathrm{C}} 75.5\right)$; from $\mathrm{H}-2^{\prime \prime}$ to $\mathrm{C}-3^{\prime}, \mathrm{C}-3^{\prime \prime}, \mathrm{C}-4^{\prime \prime}\left(\delta_{\mathrm{C}} 27.3\right)$, and $\mathrm{C}-5^{\prime \prime}\left(\delta_{\mathrm{C}} 27.3\right)$; from $\mathrm{H}-4^{\prime \prime}\left(\delta_{\mathrm{H}} 1.37,3 \mathrm{H}, \mathrm{s}\right)$ to $\mathrm{C}-3^{\prime \prime}$ and $\mathrm{C}-5^{\prime \prime}$; and from H-5" $\left(\delta_{\mathrm{H}} 1.37,3 \mathrm{H}, \mathrm{s}\right)$ to C-3". Taking into account the (-)-HRESIMS (Figure S45, Supporting Information) at $m / z 351.0878[\mathrm{M}-\mathrm{H}]^{-}$ (calcd for $\left.\mathrm{C}_{20} \mathrm{H}_{15} \mathrm{O}_{6}{ }^{-}, 351.0874\right), 12$ was elucidated as licoisoflavone B. ${ }^{10}$

Compounds 2 and 15 in GU-MF-15 were identified. Analysis of ${ }^{1} \mathrm{H}$ NMR data and HMBC spectrum (Figures S46-48 and S51, Supporting Information) of GU-MF-15 revealed the presence of two major 5-OH flavonoids: one subype I-1, 5,7-dihydroxy-8-prenyl (iso)flavonone $\left(\delta_{\mathrm{H}} 12.27,1 \mathrm{H}, \mathrm{s}, 5-\mathrm{OH} ; \delta_{\mathrm{C}} 161.4\right.$, C-5; 95.4, C-6; 102.0, C-10), and one subtype II-2, 5,7-dihydroxy (iso)flavone $\left(\delta_{\mathrm{H}} 12.91,1 \mathrm{H}, \mathrm{s}, 5-\mathrm{OH} ; \delta_{\mathrm{C}}\right.$ 161.9, C-5; 99.1, C-6; 104.3, C-10).

Analysis of the ${ }^{1} \mathrm{H}$ NMR and HSQC spectra (Figures S46-48 and S50, Supporting Information) of GU-MF-15 revealed the presence of one methylene $\left(\delta_{\mathrm{H}} 4.48,1 \mathrm{H}, \mathrm{t}, J=11.0 \mathrm{~Hz}, \mathrm{H}-2 \mathrm{a} ; 4.44,1 \mathrm{H}, \mathrm{dd}, J=\right.$ $\left.11.0,5.5 \mathrm{~Hz}, \mathrm{H}-2 \mathrm{~b} ; \delta_{\mathrm{C}} 70.0, \mathrm{C}-2\right)$ and one methine $\left(\delta_{\mathrm{H}} 4.26,1 \mathrm{H}, \mathrm{dd}, J=11.0,5.5 \mathrm{~Hz}, \mathrm{H}-3 ; \delta_{\mathrm{C}} 45.6, \mathrm{C}-3\right)$, with the same peak area for each proton signal as that of proton resonance $12.27 \mathrm{ppm}$ in 2 , indicating compound 2 was an isoflavonone. The NMR data $\left(\delta_{\mathrm{H}} 6.31,1 \mathrm{H}, \mathrm{d}, J=8.3 \mathrm{~Hz}, \mathrm{H}-5^{\prime} ; 6.67,1 \mathrm{H}, \mathrm{d}, J=8.3 \mathrm{~Hz}\right.$, H-6'; $\delta_{\mathrm{C}} 106.8$, C-5'; 126.7, C-6') showed that there was a $1^{\prime}, 2^{\prime}, 3^{\prime}, 4^{\prime}$-tetrasubstituted B-ring in 2 , which was supported by the HMBC correlations (Figure S51, Supporting Information) from H-5' to C-1' $\left(\delta_{\mathrm{C}} 113.9\right)$, C-3' $\left(\delta_{\mathrm{C}} 116.1\right)$, and C-4' $\left(\delta_{\mathrm{C}} 154.9\right)$; and from H-6' to C-3 $\left(\delta_{\mathrm{C}} 45.6\right), \mathrm{C}-2^{\prime}\left(\delta_{\mathrm{C}} 153.8\right)$, and C-4'. Considering the (-)-HRESIMS (Figure S54, Supporting Information) at $m / z 423.1779[\mathrm{M}-\mathrm{H}]^{-}$(calcd for $\mathrm{C}_{25} \mathrm{H}_{27} \mathrm{O}_{6}{ }^{-}$, 423.1813), 2 was determined as 3'-( $\gamma, \gamma$-dimethylallyl)-kievitone. ${ }^{11}$

Analysis of the ${ }^{1} \mathrm{H}$ NMR and HSQC spectra (Figures S46-48 and S50, Supporting Information) of GU-MF-15 revealed the presence of one proton signal $\left(\delta_{\mathrm{H}} 8.35,1 \mathrm{H}, \mathrm{s}, \mathrm{H}-2 ; \delta_{\mathrm{C}} 154.1\right.$, C-2 $)$ with the same peak area as that of proton resonance $12.91 \mathrm{ppm}$ in 15, indicating that compound $\mathbf{1 5}$ was an isoflavone. The NMR data $\left(\delta_{\mathrm{H}} 7.27,1 \mathrm{H}, \mathrm{d}, J=2.0 \mathrm{~Hz}, \mathrm{H}-2^{\prime} ; 6.80,1 \mathrm{H}, \mathrm{d}, J=8.2 \mathrm{~Hz}, \mathrm{H}-5^{\prime} ; 7.29,1 \mathrm{H}, \mathrm{dd}, J=8.2,2.0 \mathrm{~Hz}, \mathrm{H}-6^{\prime}\right.$; $\left.\delta_{\mathrm{C}} 126.7, \mathrm{C}-2^{\prime} ; 115.5, \mathrm{C}-5^{\prime} ; 129.5, \mathrm{C}-6^{\prime}\right)$ showed that there was a $1^{\prime}, 3^{\prime}, 4^{\prime}$-trisubstituted B-ring in $\mathbf{1 5}$, which 
was proved by the HMBC correlations (Figure S51, Supporting Information) from H-2' to C-3 $\left(\delta_{\mathrm{C}} 121.8\right)$, $\mathrm{C}-4^{\prime}\left(\delta_{\mathrm{C}} 152.3\right), \mathrm{C}-6^{\prime}\left(\delta_{\mathrm{C}} 129.5\right)$, and C-1"' $\left(\delta_{\mathrm{C}} 121.4\right)$; from H-5' to C-1' $\left(\delta_{\mathrm{C}} 123.2\right), \mathrm{C}-3^{\prime}\left(\delta_{\mathrm{C}} 120.5\right)$, and C-4'; and from H-6' to C-3, C-2' and C-4'. The NMR data $\left(\delta_{\mathrm{H}} 6.43,1 \mathrm{H}, \mathrm{d}, J=9.8 \mathrm{~Hz}, \mathrm{H}-1^{\prime \prime} ; 5.77,1 \mathrm{H}, \mathrm{d}, J=9.8\right.$ $\left.\mathrm{Hz}, \mathrm{H}-2^{\prime \prime} ; \delta_{\mathrm{C}} 121.4, \mathrm{C}-1^{\prime \prime} ; 131.2, \mathrm{C}-2^{\prime \prime}\right)$ revealed the presence of a 2,2-dimethylpyran ring in 15 with the same pattern as that of isoderrone, which was proved by the HMBC correlations (Figure S51, Supporting Information) from $\mathrm{H}-1^{\prime \prime}$ to $\mathrm{C}-4^{\prime}$ and $\mathrm{C}-3^{\prime \prime}\left(\delta_{\mathrm{C}} 76.3\right)$; from $\mathrm{H}-2^{\prime \prime}$ to $\mathrm{C}-3^{\prime}$ and $\mathrm{C}-3^{\prime \prime}$; from $\mathrm{H}-4^{\prime \prime}\left(\delta_{\mathrm{H}} 1.39,3 \mathrm{H}, \mathrm{s}\right)$ to $\mathrm{C}-2^{\prime \prime}\left(\delta_{\mathrm{C}} 131.2\right), \mathrm{C}-3^{\prime \prime}$, and C-5" $\left(\delta_{\mathrm{C}} 27.5\right)$; and from $\mathrm{H}-5^{\prime \prime}\left(\delta_{\mathrm{H}} 1.39,3 \mathrm{H}, \mathrm{s}\right)$ to C-2", $\mathrm{C}-3^{\prime \prime}$, and C-4" $\left(\delta_{\mathrm{C}}\right.$ 27.5). Considering the (-)-HRESIMS (Figure S53, Supporting Information) at $\mathrm{m} / z 335.0893[\mathrm{M}-\mathrm{H}]^{-}$ (calcd for $\mathrm{C}_{20} \mathrm{H}_{15} \mathrm{O}_{5}{ }^{-}, 335.0925$ ), 15 was determined as isoderrone. ${ }^{10}$

Compound 5 was identified in GU-MF-16. The ${ }^{1} \mathrm{H}$ NMR data and HMBC spectrum (Figures S55, S56, and S59, Supporting Information) revealed the presence of a major 5-OH flavonoid: one subtype II-3, 5,7-dihydroxy-6-prenyl (iso)flavone ( $\delta_{\mathrm{H}} 13.26,1 \mathrm{H}, \mathrm{s}, 5-\mathrm{OH} ; \delta_{\mathrm{C}} 158.8, \mathrm{C}-5 ; 111.1, \mathrm{C}-6$; 104.1, C-10). Analysis of the ${ }^{1} \mathrm{H}$ NMR and HSQC spectra (Figures S55, S56, and S58, Supporting Information) of GU-MF-16 revealed the presence of one proton signal $\left(\delta_{\mathrm{H}} 8.20,1 \mathrm{H}, \mathrm{s}, \mathrm{H}-2 ; \delta_{\mathrm{C}} 153.4\right.$, C-2 $)$ with the same peak area as that of proton resonance $13.26 \mathrm{ppm}$ in $\mathbf{5}$, indicating that compound $\mathbf{5}$ was an isoflavone. Analysis of the NMR data $\left(\delta_{\mathrm{H}} 6.88,1 \mathrm{H}\right.$, brs, H-2'; 6.67, $\left.1 \mathrm{H}, \mathrm{brs}, \mathrm{H}-6^{\prime} ; \delta_{\mathrm{C}} 113.9, \mathrm{C}-2^{\prime} ; 120.3, \mathrm{C}-6^{\prime}\right)$ revealed that there was a $1^{\prime}, 3^{\prime}, 4^{\prime}, 5^{\prime}$-tetrasubstituted B-ring in 5, which was proved by the HMBC correlations (Figure S59, Supporting Information) from H-2' to C-3' $\left(\delta_{\mathrm{C}} 144.2\right), \mathrm{C}-4^{\prime}\left(\delta_{\mathrm{C}} 143.1\right)$, and C-6 $\left(\delta_{\mathrm{C}} 120.3\right)$; and from H-6' to C-2' $\left(\delta_{\mathrm{C}} 113.9\right), \mathrm{C}-4^{\prime}$, and C-1"' $\left(\delta_{\mathrm{C}} 28.2\right)$. Taking into account the (-)-HRESIMS (Figure S61, Supporting Information) at $m / z, 421.1639[\mathrm{M}-\mathrm{H}]^{-}$(calcd for $\mathrm{C}_{25} \mathrm{H}_{25} \mathrm{O}_{6}{ }^{-}, 421.1657$ ), 5 was determined as isoangustone A. ${ }^{10}$

Compound 6 was identified in GU-MF-17. The ${ }^{1} \mathrm{H}$ NMR data and HMBC spectrum (Figures S62, S63, and S66, Supporting Information) revealed the presence of a major 5-OH flavonoid: one subtype II-3, 5,7-dihydroxy-6-prenyl (iso)flavone $\left(\delta_{\mathrm{H}} 13.21,1 \mathrm{H}, \mathrm{s}, 5-\mathrm{OH} ; \delta_{\mathrm{C}} 158.9, \mathrm{C}-5 ; 111.2, \mathrm{C}-6 ; 104.2, \mathrm{C}-10\right)$.

Analysis of the ${ }^{1} \mathrm{H}$ NMR and HSQC spectra (Figures S62, S63, and S65, Supporting Information) of GU-MF-17 revealed the presence of one proton signal $\left(\delta_{\mathrm{H}} 8.30,1 \mathrm{H}, \mathrm{s}, \mathrm{H}-2 ; \delta_{\mathrm{C}} 153.7, \mathrm{C}-2\right)$ with the same peak area as that of proton resonance $13.21 \mathrm{ppm}$ in $\mathbf{6}$, indicating that compound $\mathbf{6}$ was an isoflavone. The NMR data $\left(\delta_{\mathrm{H}} 6.91\right.$ and 6.72 , each $1 \mathrm{H}$, brs, H-2' and $\mathrm{H}-6^{\prime} ; \delta_{\mathrm{C}} 116.8$ and $117.3, \mathrm{C}-2^{\prime}$ and $\left.\mathrm{C}-6^{\prime}\right)$ revealed that 
there was a 1',3',4',5'-tetrasubstituted B-ring in 6, which was proved by the HMBC correlations (Figure S66, Supporting Information) from H-2' to C-3' $\left(\delta_{\mathrm{C}} 145.2\right), \mathrm{C}-4^{\prime}\left(\delta_{\mathrm{C}} 140.0\right)$, and C-6' $\left(\delta_{\mathrm{C}} 117.3\right)$; and from H-6' to C-2' $\left(\delta_{\mathrm{C}} 116.8\right), \mathrm{C}-4^{\prime}$, and C-1"' $\left(\delta_{\mathrm{C}} 121.9\right)$. The NMR data $\left(\delta_{\mathrm{H}} 6.38,1 \mathrm{H}, \mathrm{d}, J=10.0 \mathrm{~Hz}, \mathrm{H}-1^{\prime \prime} ; 5.75,1 \mathrm{H}, \mathrm{d}\right.$, $J=10.0 \mathrm{~Hz}, \mathrm{H}-2^{\prime \prime} ; \delta_{\mathrm{C}} 121.9$ and 131.1$)$ revealed that 6 possessed a 2,2-dimethylpyran ring with the same pattern as that of 14. Considering the (-)-HRESIMS (Figure S68, Supporting Information) at $m / z 419.1490$ $[\mathrm{M}-\mathrm{H}]^{-}$(calcd for $\mathrm{C}_{25} \mathrm{H}_{23} \mathrm{O}_{6}{ }^{-}, 419.1500$ ), compound 6 was determined as gancaonin $\mathrm{H}^{12}$

A major component of GU-MF-18 was identified as $1\left(\delta_{\mathrm{H}} 12.56,1 \mathrm{H}, \mathrm{s}, 5-\mathrm{OH} ; \delta_{\mathrm{C}} 161.0, \mathrm{C}-5 ; 107.6, \mathrm{C}-6\right.$; 101.7, C-10), one new 5,7-dihydroxy-6-prenyl (iso)flavonone (subtype I-3), based on the analysis of the ${ }^{1} \mathrm{H}$ NMR and HMBC spectra (Figures S69, S70, and S73, Supporting Information). Analysis of the ${ }^{1} \mathrm{H}$ NMR and HSQC spectra of GU-MF-18 (Figures S69, S70, and S72, Supporting Information) revealed the presence of one methylene $\left(\delta_{\mathrm{H}} 4.47,1 \mathrm{H}, \mathrm{t}, J=11.0 \mathrm{~Hz}, \mathrm{H}-2 \mathrm{a} ; 4.38,1 \mathrm{H}, \mathrm{dd}, J=11.0,5.5 \mathrm{~Hz}, \mathrm{H}-2 \mathrm{~b} ; \delta_{\mathrm{C}} 69.7\right.$, C-2), one $\mathrm{sp}^{3}$ methine $\left(\delta_{\mathrm{H}} 4.26,1 \mathrm{H}, \mathrm{dd}, J=11.0,5.5 \mathrm{~Hz}, \mathrm{H}-3 ; \delta_{\mathrm{C}} 46.2, \mathrm{C}-3\right)$, one $\mathrm{sp}^{2}$ methine $\left(\delta_{\mathrm{H}} 5.98,1 \mathrm{H}, \mathrm{s}\right.$, $\left.\mathrm{H}-8 ; \delta_{\mathrm{C}} 94.2, \mathrm{C}-8\right)$, two AB-coupled aromatic hydrogens $\left(\delta_{\mathrm{H}} 6.27,1 \mathrm{H}, \mathrm{d}, J=8.2 \mathrm{~Hz}, \mathrm{H}-5^{\prime} ; 6.82,1 \mathrm{H}, \mathrm{d}, J=\right.$ $\left.8.2 \mathrm{~Hz}, \mathrm{H}-6^{\prime} ; \delta_{\mathrm{C}} 107.7, \mathrm{C}-5^{\prime} ; 130.1, \mathrm{C}-6^{\prime}\right)$, and two olefinic hydrogens $\left(\delta_{\mathrm{H}} 6.68,1 \mathrm{H}, \mathrm{d}, J=10.0 \mathrm{~Hz}, \mathrm{H}-1^{\prime \prime \prime}\right.$; $\left.5.67,1 \mathrm{H}, \mathrm{d}, J=10.0 \mathrm{~Hz}, \mathrm{H}-2^{\prime \prime \prime} ; \delta_{\mathrm{C}} 116.9, \mathrm{C}-1^{\prime \prime \prime} ; 129.0, \mathrm{C}-2^{\prime \prime \prime}\right)$, with the same resonance area for each proton signal as that of the $\delta_{\mathrm{H}} 12.56 \mathrm{LFR}$ singlet in $\mathbf{1}$, indicating compound $\mathbf{1}$ was an isoflavonone. The COSY spectrum confirmed the presence of four spin systems (bold bonds in Figure S71, Supporting Information). The HMBC correlations (Figure S73, Supporting Information) from $\mathrm{H}-5^{\prime}$ to $\mathrm{C}-1^{\prime}\left(\delta_{\mathrm{C}} 115.6\right)$ and C-3' $\left(\delta_{\mathrm{C}} 110.1\right)$; from H-6' to C-3 $\left(\delta_{\mathrm{C}} 46.2\right), \mathrm{C}-2^{\prime}\left(\delta_{\mathrm{C}} 150.6\right)$ and C-4' $\left(\delta_{\mathrm{C}} 152.5\right)$; from H-1"' to C-4' and C-3"' $\left(\delta_{\mathrm{C}} 75.1\right)$; from H-2'" to C-3' and C-3"'; from H-4"' to C-2"' $\left(\delta_{\mathrm{C}} 129.0\right), \mathrm{C}-3^{\prime \prime \prime}$, and C-5"' $\left(\delta_{\mathrm{C}} 27.4\right)$; and from $\mathrm{H}-5^{\prime \prime \prime}$ to $\mathrm{C}-2^{\prime \prime \prime}$ and $\mathrm{C}-3^{\prime \prime \prime}$ revealed that 1 was closely related to 3 having the same B-ring. Their difference was the presence vs lack of a C-6 prenyl substituent in $\mathbf{1}$ vs 3. Elucidation of $\mathbf{1}$ as the new 6-prenyl licoisoflavanone was ultimately supported by the molecular formula $\left(\mathrm{C}_{25} \mathrm{H}_{26} \mathrm{O}_{6}\right)$ of $\mathbf{1}$ assigned via (-)-HRESIMS (Figure S75, Supporting Information) at $m / z 421.1654[\mathrm{M}-\mathrm{H}]^{-}$(calcd for $\mathrm{C}_{25} \mathrm{H}_{25} \mathrm{O}_{6}{ }^{-}$, 421.1657), reflecting an increase of one prenyl group. This was corroborated by concentrating 1 from GU-MF-18 via Sephadex LH-20 gel chromatography and subsequent NMR and MS data analysis of GU-MF-18-1 (Figures S76-83, Supporting Information). 
Compound 9 was identified in GU-MF-19. The ${ }^{1} \mathrm{H}$ NMR data and HMBC spectrum (Figures S84, S85, and S88, Supporting Information) revealed the presence of a major 5-OH flavonoid $\left(\delta_{\mathrm{H}} 13.21,1 \mathrm{H}, \mathrm{s}, 5-\mathrm{OH}\right.$; $\delta_{\mathrm{C}} \mathrm{C}-5,156.7$; C-6, 111.7; C-10, 104.6), which showed high similarity to those of subtype II-3, 5,7-dihydroxy-6-prenyl (iso)flavone. Comparison of their NMR data indicated that the C-5 was shielded by $\Delta \delta_{\mathrm{C}}-2.1$ in 9 , suggesting that there is likely an additional 8-prenyl group in 9 . The ${ }^{1} \mathrm{H}$ NMR data $\left(\delta_{\mathrm{H}}\right.$ $8.38,1 \mathrm{H}, \mathrm{s}, \mathrm{H}-2)$ with the same peak area as that of proton resonance $13.21 \mathrm{ppm}$ in 9 revealed that compound 9 was an isoflavone. Analysis of the ${ }^{1} \mathrm{H}$ NMR data and HSQC spectrum $\left(\delta_{\mathrm{H}} 7.38,2 \mathrm{H}, \mathrm{d}, J=8.2\right.$ $\mathrm{Hz}, \mathrm{H}-2^{\prime}, 6^{\prime} ; 6.82,2 \mathrm{H}$, brd, $J=8.2 \mathrm{~Hz}, \mathrm{H}-3^{\prime}, 5^{\prime} ; \delta_{\mathrm{C}} 130.2$ and $115.1, \mathrm{C}-2^{\prime}, 6^{\prime}$ and $\left.\mathrm{C}-3^{\prime}, 5^{\prime}\right)$ revealed that compound 9 had a 4'-hydroxy substituted B-ring (Figures S84, S85, and S87, Supporting Information), which was proved by the COSY correlations (Figure S86, Supporting Information) of H-2'/H-3' and H-5'/H-6', and HMBC correlations (Figure S88, Supporting Information) from H-2' and H-6' to C-4' ( $\delta_{\mathrm{C}}$ 157.4). Considering the (-)-HRESIMS (Figure S90, Supporting Information) at $\mathrm{m} / z 405.1704[\mathrm{M}-\mathrm{H}]^{-}$ (calcd for $\mathrm{C}_{25} \mathrm{H}_{25} \mathrm{O}_{5}{ }^{-}, 405.1707$ ), 9 was determined as 6,8-diprenylgenistein. ${ }^{13}$

Compounds 4 and 7 were identified in GU-MF-20. The ${ }^{1} \mathrm{H}$ NMR data and HMBC spectrum (Figures S91-93 and S96, Supporting Information) revealed the presence of two major 5-OH flavonoids: two subtype II-3, 5,7-dihydroxy-6-prenyl (iso)flavone $\left(\delta_{\mathrm{H}} 13.10,1 \mathrm{H}, \mathrm{s}, 5-\mathrm{OH} ; \delta_{\mathrm{C}} 158.6\right.$, C-5; 111.0, C-6; 104.2, $\left.\mathrm{C}-10 ; \delta_{\mathrm{H}} 13.17,1 \mathrm{H}, \mathrm{s}, 5-\mathrm{OH} ; \delta_{\mathrm{C}} 159.0, \mathrm{C}-5 ; 111.2, \mathrm{C}-6 ; 104.1, \mathrm{C}-10\right)$. Analysis of the ${ }^{1} \mathrm{H}$ NMR data and HSQC spectrum (Figures S91-93 and S95, Supporting Information) of GU-MF-20 revealed the presence of two proton signals $\left(\delta_{\mathrm{H}} 8.16,8.33\right.$, each $\left.1 \mathrm{H}, \mathrm{s}, \mathrm{H}-2\right)$ possessing the same peak areas as those of proton resonances $13.10 \mathrm{ppm}$ in $\mathbf{4}$ and $13.17 \mathrm{ppm}$ in $\mathbf{7}$, respectively, indicating that both of $\mathbf{4}$ and $\mathbf{7}$ are isoflavones.

Based on the analysis of the ${ }^{1} \mathrm{H}-{ }^{1} \mathrm{H}$ COSY spectrum (Figure S94, Supporting Information), two AB-coupled aromatic hydrogens $\left(\delta_{\mathrm{H}} 6.32,1 \mathrm{H}, \mathrm{d}, J=8.3 \mathrm{~Hz}, \mathrm{H}-5^{\prime}\right.$ and $6.88,1 \mathrm{H}$, brd, $J=8.3 \mathrm{~Hz}, \mathrm{H}-6^{\prime} ; \delta_{\mathrm{C}}$ 107.4 and 131.2, C-5' and C-6') in B-ring for 4, and three ABX-coupled aromatic hydrogens $\left(\delta_{\mathrm{H}} 7.26,1 \mathrm{H}, \mathrm{d}\right.$, $J=2.3 \mathrm{~Hz}, \mathrm{H}-2^{\prime} ; 6.79,1 \mathrm{H}, \mathrm{d}, J=8.3 \mathrm{~Hz}, \mathrm{H}-5^{\prime} ; 7.29,1 \mathrm{H}, \mathrm{dd}, J=8.3,2.3 \mathrm{~Hz}, \mathrm{H}-6^{\prime} ; \delta_{\mathrm{C}} 126.8, \mathrm{C}-2^{\prime} ; 115.5, \mathrm{C}^{\prime} 5^{\prime}$; 129.6, C-6') in B-ring for 7 were decided. Thus, the $1^{\prime}, 2^{\prime}, 3^{\prime}, 4^{\prime}$-tetrasubstituted B-ring in 4 and $1^{\prime}, 3^{\prime}, 4^{\prime}$-trisubstituted B-ring in 7 were determined, which was proved by the HMBC correlations (Figure S96, Supporting Information) from $\mathrm{H}-5^{\prime}$ to $\mathrm{C}-1^{\prime}\left(\delta_{\mathrm{C}} 111.3\right)$ and $\mathrm{C}-3^{\prime}\left(\delta_{\mathrm{C}} 110.0\right)$; and from $\mathrm{H}-6^{\prime}$ to $\mathrm{C}-2^{\prime}\left(\delta_{\mathrm{C}}\right.$ $151.1)$ in 4 ; from $\mathrm{H}-2^{\prime}$ to $\mathrm{C}-6^{\prime}\left(\delta_{\mathrm{C}} 129.6\right)$; and from $\mathrm{H}-6^{\prime}$ to $\mathrm{C}-2^{\prime}\left(\delta_{\mathrm{C}} 126.8\right)$ in 7 . There were also double 
bonds in $4\left(\delta_{\mathrm{H}} 6.67,1 \mathrm{H}, \mathrm{d}, J=9.8 \mathrm{~Hz}, \mathrm{H}-1^{\prime \prime \prime} ; 5.68,1 \mathrm{H}, \mathrm{d}, J=9.8 \mathrm{~Hz}, \mathrm{H}-2^{\prime \prime \prime} ; \delta_{\mathrm{C}} 116.9\right.$ and $128.8, \mathrm{C}-1^{\prime \prime \prime}$ and $\left.\mathrm{C}-2^{\prime \prime \prime}\right)$ and $7\left(\delta_{\mathrm{H}} 6.43,1 \mathrm{H}, \mathrm{d}, J=9.8 \mathrm{~Hz}, \mathrm{H}-1^{\prime \prime \prime} ; 5.78,1 \mathrm{H}, \mathrm{d}, J=9.8 \mathrm{~Hz}, \mathrm{H}-2^{\prime \prime \prime} ; \delta_{\mathrm{C}} 121.5\right.$ and $131.2, \mathrm{C}-1^{\prime \prime \prime}$ and C-2'"'), indicating both of 4 and 7 possessed 2,2-dimethylpyran rings with the same patterns as those of 12 and 15, respectively, which was proved by the HMBC correlations (Figure S96, Supporting Information) from $\mathrm{H}-1^{\prime \prime \prime}$ to $\mathrm{C}-3^{\prime \prime \prime}\left(\delta_{\mathrm{C}} 75.3\right)$; from $\mathrm{H}-2^{\prime \prime \prime}$ to $\mathrm{C}-3^{\prime}\left(\delta_{\mathrm{C}} 110.0\right)$ and $\mathrm{C}-3^{\prime \prime \prime}$; from $\mathrm{H}-4^{\prime \prime \prime}$ to $\mathrm{C}-2^{\prime \prime \prime}\left(\delta_{\mathrm{C}} 128.8\right)$, C-3"', and C-5"' $\left(\delta_{\mathrm{C}} 27.5\right)$; and from H-5"' to C-2"' and C-3"' in 4; from H-1"' to C-3"' $\left(\delta_{\mathrm{C}} 76.4\right)$; from $\mathrm{H}-2^{\prime \prime \prime}$ to $\mathrm{C}-3^{\prime}\left(\delta_{\mathrm{C}} 120.5\right)$ and C-3"'”; from H-4"' to C-2"' $\left(\delta_{\mathrm{C}} 131.2\right), \mathrm{C}-3^{\prime \prime \prime}$, and C-5"' $\left(\delta_{\mathrm{C}} 27.6\right)$; and from H-5"' to C-2"' and C-3"' in 7. Considering the (-)-HRESIMS (Figures S98 and S99, Supporting Information) at $m / z 419.1476[\mathrm{M}-\mathrm{H}]^{-}$(calcd for $\left.\mathrm{C}_{25} \mathrm{H}_{23} \mathrm{O}_{6}{ }^{-}, 419.1500\right)$ and $m / z 403.1537[\mathrm{M}-\mathrm{H}]^{-}$(calcd for $\mathrm{C}_{25} \mathrm{H}_{23} \mathrm{O}_{5}{ }^{-}, 403.1551$ ) for $\mathbf{4}$ and 7 , respectively, their structures were determined as angustone $\mathrm{B}^{14}$ for 4 and isochandalone ${ }^{15}$ for 7. 


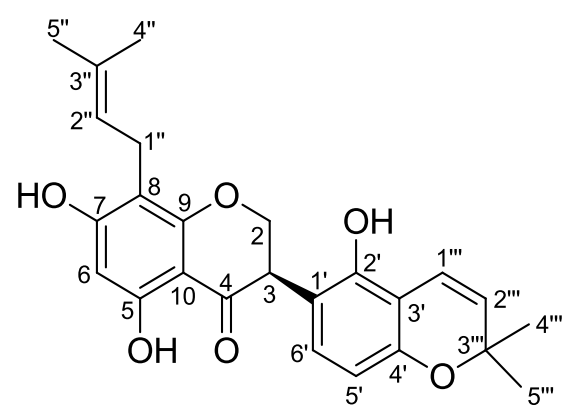

A

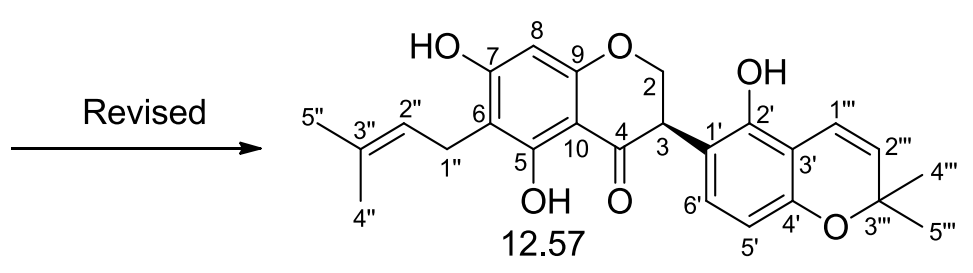

B

Figure S1. Original structure of licoisoflavanone B reported in the literature (A) vs its revised structure determined as relicoisoflavanone B (B).<smiles>[B-]C1(C)C=Cc2c(ccc(C3COc4cc(O)c(CC=C(C)C)c(O)c4C3=O)c2O)O1</smiles>

A

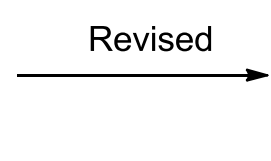

Figure S2. Original structure of licoiso
determined as relicoisoflavanone C (B).

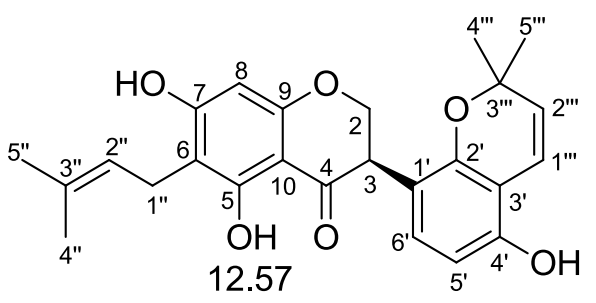

B 


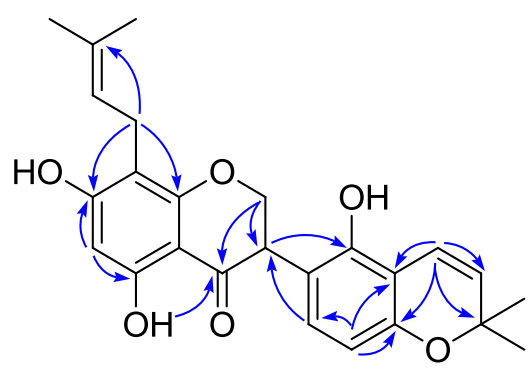

A

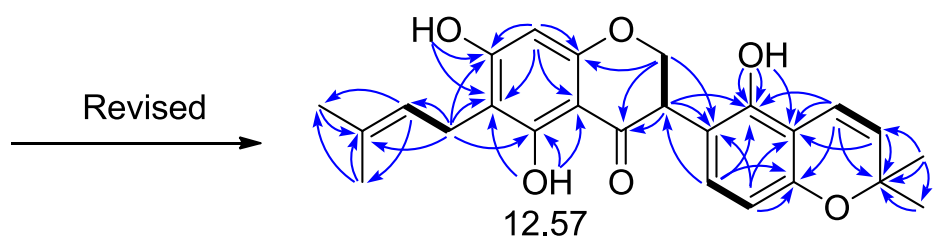

B

Figure S3. ${ }^{1} \mathrm{H}-{ }^{1} \mathrm{H}$ COSY $(-)$ and selected $\operatorname{HMBC}(\mathrm{H} \rightarrow \mathrm{C})$ correlations of the original structure of licoisoflavanone B reported in the literature (A) vs its revised structure determined as relicoisoflavanone B (B).

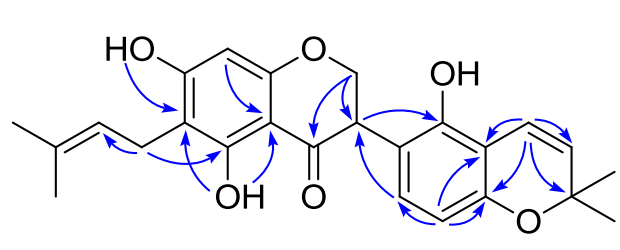

A

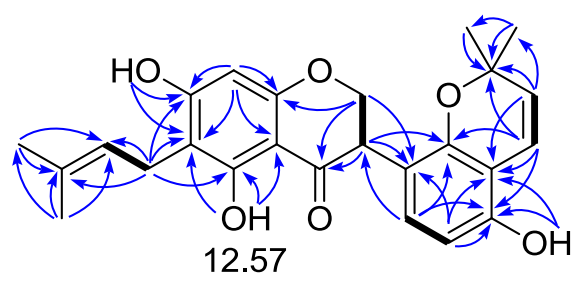

B

Figure S4. ${ }^{1} \mathrm{H}-{ }^{1} \mathrm{H}$ COSY $(-)$ and selected $\mathrm{HMBC}(\mathrm{H} \rightarrow \mathrm{C})$ correlations of the original structure of licoisoflavanone $\mathrm{C}$ reported in the literature (A) vs its revised structure determined as relicoisoflavanone $\mathrm{C}$ (B). 
SECTION S4. STRUCTURE ELUCIDATION AND VERIFICATION RELATED TABLES.

Table S1. Comparison of the ${ }^{1} \mathrm{H}$ and ${ }^{13} \mathrm{C}$ NMR spectroscopic data of licoisoflavanone B vs relicoisoflavanone B, compound 1, and licoisoflavanone $\mathrm{C}$ vs relicoisoflavanone $\mathrm{C}$ (all in DMSO- $d_{6}$ ).

\begin{tabular}{|c|c|c|c|c|c|c|c|c|c|c|}
\hline & \multicolumn{2}{|c|}{ licoisoflavanone $\mathrm{B}^{a}$} & \multicolumn{2}{|c|}{ relicoisoflavanone $\mathrm{B}^{b}$} & \multicolumn{2}{|l|}{$\mathbf{1}$} & \multicolumn{2}{|c|}{ licoisoflavanone $\mathrm{C}^{a}$} & \multicolumn{2}{|c|}{ relicoisoflavanone $\mathrm{C}^{b}$} \\
\hline 2 & $\begin{array}{l}\delta_{\mathrm{H}} \\
4.36 \mathrm{~m} \\
4.46 \mathrm{~m}\end{array}$ & $\begin{array}{l}\delta_{\mathrm{C}} \\
70.2\end{array}$ & $\begin{array}{l}\delta_{\mathrm{H}} \\
4.36 \mathrm{~m} \\
4.46 \mathrm{~m}\end{array}$ & $\begin{array}{l}\delta_{\mathrm{C}} \\
70.2\end{array}$ & $\begin{array}{l}\delta_{\mathrm{H}^{c}} \\
4.38 \mathrm{dd}(11.0,5.5) \\
4.47 \mathrm{t}(11.0)\end{array}$ & $\begin{array}{l}\delta_{\mathrm{C}^{d}} \\
69.7\end{array}$ & $\begin{array}{l}\delta_{\mathrm{H}} \\
4.33 \mathrm{~m}\end{array}$ & $\begin{array}{l}\delta_{\mathrm{C}} \\
70.2\end{array}$ & $\begin{array}{l}\delta_{\mathrm{H}} \\
4.33 \mathrm{~m}\end{array}$ & $\begin{array}{l}\delta_{\mathrm{C}} \\
70.3\end{array}$ \\
\hline 3 & $4.25 \mathrm{q}$ & 46.8 & $4.25 \mathrm{q}$ & 46.8 & $4.26 \mathrm{dd}(11.0,5.5)$ & 46.2 & $4.13 \mathrm{q}$ & 46.5 & $4.13 \mathrm{q}$ & 46.5 \\
\hline 4 & & 197.9 & & 197.9 & & 197.3 & & 197.8 & & 197.8 \\
\hline 5 & & 164.4 & & 161.4 & & 161.0 & & 164.2 & & 161.3 \\
\hline 6 & $5.98 \mathrm{~s}$ & 94.6 & & 108.1 & & 107.6 & & 108.0 & & 108.0 \\
\hline 7 & & 161.4 & & 164.4 & & 163.9 & & 161.3 & & 164.2 \\
\hline 8 & & 108.1 & $5.98 \mathrm{~s}$ & 94.6 & $5.96 \mathrm{~s}$ & 94.2 & $5.97 \mathrm{~s}$ & 94.4 & $5.97 \mathrm{~s}$ & 94.6 \\
\hline 9 & & 161.1 & & 161.1 & & 160.5 & & 161.0 & & 161.0 \\
\hline 10 & & 102.1 & & 102.1 & & 101.7 & & 102.5 & & 102.5 \\
\hline $1^{\prime}$ & & 116.1 & & 116.1 & & 115.6 & & 109.4 & & 113.8 \\
\hline $2^{\prime}$ & & 151.2 & & 151.2 & & 150.6 & & 151.1 & & 151.3 \\
\hline $3^{\prime}$ & & 110.6 & & 110.6 & & 110.1 & & 113.8 & & 109.4 \\
\hline $4^{\prime}$ & & 153.1 & & 153.1 & & 152.5 & & 153.1 & & 153.1 \\
\hline $5^{\prime}$ & $6.26 \mathrm{~d}(8.4)$ & 108.3 & $6.26 \mathrm{~d}(8.4)$ & 108.3 & $6.27 \mathrm{~d}(8.4)$ & 107.7 & $6.32 \mathrm{~d}(8.0)$ & 108.0 & $6.32 \mathrm{~d}(8.0)$ & 108.0 \\
\hline $6^{\prime}$ & $6.81 \mathrm{~d}(8.4)$ & 130.5 & $6.81 \mathrm{~d}(8.4)$ & 130.5 & $6.82 \mathrm{~d}(8.4)$ & 130.1 & $6.78 \mathrm{~d}(8.0)$ & 130.6 & $6.78 \mathrm{~d}(8.0)$ & 130.6 \\
\hline $1^{\prime \prime}$ & $3.12 \mathrm{~d}(6.8)$ & 21.1 & $3.12 \mathrm{~d}(6.8)$ & 21.1 & $3.11 \mathrm{~d}(7.0)$ & 20.7 & $3.11 \mathrm{~d}(6.8)$ & 21.1 & $3.11 \mathrm{~d}(6.8)$ & 21.1 \\
\hline $2^{\prime \prime}$ & $5.13 \mathrm{t}(6.4)$ & 123.1 & $5.13 \mathrm{t}(6.4)$ & 123.1 & $5.13 \mathrm{tt}(7.0,1.2)$ & 122.6 & $5.12 \mathrm{t}(6.4)$ & 123.1 & $5.12 \mathrm{t}(6.4)$ & 123.1 \\
\hline $3^{\prime \prime}$ & & 130.7 & & 130.7 & & 130.1 & & 125.8 & & 130.6 \\
\hline $4^{\prime \prime}$ & $1.69 \mathrm{~s}$ & 18.1 & $1.69 \mathrm{~s}$ & 18.1 & $1.69 \mathrm{~s}$ & 17.6 & $1.69 \mathrm{~s}$ & 25.9 & $1.61 \mathrm{~s}$ & 25.9 \\
\hline $5^{\prime \prime}$ & $1.62 \mathrm{~s}$ & 25.9 & $1.62 \mathrm{~s}$ & 25.9 & $1.61 \mathrm{~s}$ & 25.4 & $1.61 \mathrm{~s}$ & 18.1 & $1.69 \mathrm{~s}$ & 18.1 \\
\hline $1^{\prime \prime \prime}$ & $6.67 \mathrm{~d}(10.0)$ & 117.4 & $6.67 \mathrm{~d}(10.0)$ & 117.4 & $6.67 \mathrm{~d}(10.0)$ & 116.9 & $6.54 \mathrm{~d}(10.0)$ & 117.2 & $6.54 \mathrm{~d}(10.0)$ & 117.2 \\
\hline $2^{\prime \prime \prime}$ & $5.66 \mathrm{~d}(10.0)$ & 129.5 & $5.66 \mathrm{~d}(10.0)$ & 129.5 & $5.68 \mathrm{~d}(10.0)$ & 129.0 & $5.59 \mathrm{~d}(10.0)$ & 128.8 & $5.59 \mathrm{~d}(10.0)$ & 128.8 \\
\hline $3^{\prime \prime \prime}$ & & 75.5 & & 75.5 & & 75.1 & & 76.3 & & 76.3 \\
\hline $4^{\prime \prime \prime}$ & $1.34 \mathrm{~s}$ & 27.8 & $1.34 \mathrm{~s}$ & 27.8 & $1.34 \mathrm{~s}$ & 27.4 & $1.23 \mathrm{~s}$ & 27.5 & $1.23 \mathrm{~s}$ & 27.5 \\
\hline $5^{\prime \prime \prime}$ & $1.34 \mathrm{~s}$ & 27.8 & $1.34 \mathrm{~s}$ & 27.8 & $1.34 \mathrm{~s}$ & 27.4 & $1.23 \mathrm{~s}$ & 27.9 & $1.23 \mathrm{~s}$ & 27.9 \\
\hline $\mathrm{OH}$ & $12.57 \mathrm{~s}$ & & $12.57 \mathrm{~s}$ & & $12.56 \mathrm{~s}$ & & $12.57 \mathrm{~s}$ & & $12.57 \mathrm{~s}$ & \\
\hline $\mathrm{OH}$ & $10.74 \mathrm{~s}$ & & $10.74 \mathrm{~s}$ & & & & $10.73 \mathrm{~s}$ & & $10.73 \mathrm{~s}$ & \\
\hline $\mathrm{OH}$ & $9.17 \mathrm{~s}$ & & $9.17 \mathrm{~s}$ & & & & $9.64 \mathrm{~s}$ & & $9.64 \mathrm{~s}$ & \\
\hline
\end{tabular}

${ }^{a}$ Data were measured in $400 \mathrm{MHz}$ for ${ }^{1} \mathrm{H}$ and $100 \mathrm{MHz}$ for ${ }^{13} \mathrm{C}$

${ }^{b}$ Relicoisoflavanone $\mathrm{B}$ is the revised structure of licoisoflavanone $\mathrm{B}$; relicoisoflavanone $\mathrm{C}$ is the revised structure of licoisoflavanone $\mathrm{C}$.

${ }^{c}$ The resonance data were obtained from the ${ }^{1} \mathrm{H}$ NMR spectrum of GU-MF-18-1 using the residual solvent signals $\left(\delta_{\mathrm{H}} 2.50\right)$ as a reference.

${ }^{d}$ The resonance data were extracted from the HSQC and HMBC spectra of GU-MF-18-1, and chemical shifts were referenced to the solvent peaks $\left(\delta_{\mathrm{C}} 39.51\right)$. 
Table S2. The HMBC patterns of licoisoflavanone B vs relicoisoflavanone B, compound 1, and licoisoflavanone $\mathrm{C}$ vs relicoisoflavanone $\mathrm{C}$ (all in DMSO- $d_{6}$ ).

\begin{tabular}{lllllll}
\hline Compound Name & Structural Type & $\mathrm{MHz}\left({ }^{1} \mathrm{H}\right)$ & \multicolumn{3}{c}{ Chemical shift } \\
& & & $5-\mathrm{OH}$ & $\mathrm{C}-5$ & $\mathrm{C}-6$ & $\mathrm{C}-10$ \\
\hline licoisoflavanone B $^{*}$ & Type I-1 & 400 & 12.57 & 164.4 & 94.6 & 102.1 \\
relicoisoflavanone B & Type I-3 & 400 & 12.57 & 161.4 & 108.1 & 102.1 \\
& Type I-3 & 600 & 12.56 & 161.0 & 107.6 & 101.7 \\
licoisoflavanone C & Type I-3 & 400 & 12.57 & 164.2 & 108.0 & 102.5 \\
relicoisoflavanone C & Type I-3 & 400 & 12.57 & 161.3 & 108.0 & 102.5 \\
\hline
\end{tabular}

*Chemical shifts were referenced to 40.00 ppm of DMSO- $d_{6}$ as reported in reference 26.<smiles>CC(C)=CCc1c(O)cc2c(c1O)C(=O)C(c1ccc3c(c1O)C=CC(C)(C)O3)CO2</smiles>

relicoisoflavanone $\mathrm{B}$

(1)<smiles>CC(C)=CCc1c(O)cc2c(c1O)C(=O)C(c1ccc(O)c3c1OC(C)(C)C=C3)CO2</smiles>

relicoisoflavanone $\mathrm{C}$ 
Table S3. The HMBC patterns mined from literature data of compounds isolated from licorice species (GU, GG, and GI).

\begin{tabular}{|c|c|c|c|c|c|c|c|c|c|c|c|}
\hline \multirow[t]{2}{*}{ No. } & \multirow[t]{2}{*}{ Name Given in the Literature } & \multirow{2}{*}{$\begin{array}{l}\text { Structural } \\
\text { Type }\end{array}$} & \multirow[t]{2}{*}{ Solvent } & \multirow{2}{*}{$\begin{array}{l}\mathrm{MHz} \\
\left({ }^{1} \mathrm{H}\right)\end{array}$} & \multicolumn{4}{|c|}{ Chemical shift } & \multicolumn{3}{|c|}{ References } \\
\hline & & & & & $\begin{array}{l}5-\mathrm{OH} / \\
2^{\prime}-\mathrm{OH}\end{array}$ & $\begin{array}{l}\text { C-5/ } \\
\text { C-2' }\end{array}$ & $\begin{array}{l}C-6 / \\
C-3 '\end{array}$ & $\begin{array}{l}\text { C-10/ } \\
\text { C-1' }\end{array}$ & $\begin{array}{l}\text { Plant } \\
\text { source(s) }\end{array}$ & Isolation & $\begin{array}{l}\text { NMR } \\
\text { data }\end{array}$ \\
\hline 1 & sophoraflavanone B & Type I-1 & DMSO- $d_{6}$ & $400 / 600$ & 12.08 & 161.1 & 95.2 & 101.7 & GU,GI & 16,17 & 18 \\
\hline 2 & pinocembrin & Type I-2 & DMSO- $d_{6}$ & 500 & 12.10 & 163.5 & 95.9 & 101.8 & GU,GG & 19,20 & 21 \\
\hline 3 & sigmoidin $\mathrm{B}$ & Type I-2 & DMSO- $d_{6}$ & 300 & 12.12 & 163.5 & 95.7 & 101.8 & GU & 22 & 23 \\
\hline 4 & naringenin & Type I-2 & DMSO- $d_{6}$ & 200 & 12.20 & 163.8 & 95.3 & 101.7 & GU,GG,GI & $24,25,26$ & 27 \\
\hline 5 & licoflavanone & Type I-2 & DMSO- $d_{6}$ & 300 & 12.21 & 163.5 & 95.9 & 101.7 & GU,GG & 28,29 & 30 \\
\hline 6 & licoisoflavanone & Type I-2 & DMSO- $d_{6}$ & 400 & 12.29 & 163.9 & 96.0 & 102.0 & GU,GG & 31,32 & 10 \\
\hline 7 & 6-prenylnaringenin & Type I-3 & DMSO- $d_{6}$ & $400 / 600$ & 12.41 & 160.5 & 107.5 & 101.6 & GU,GG,GI & $28,33,17$ & 18 \\
\hline 8 & $2^{\prime}$-hydroxyisolupalbigenin & Type II-1 & DMSO- $d_{6}$ & 400 & 12.79 & 159.3 & 98.4 & 104.6 & GU,GI & 10,26 & 10 \\
\hline 9 & licoflavone $\mathrm{C}$ & Type II-1 & DMSO- $d_{6}$ & 400 & 12.90 & 159.5 & 98.8 & 104.2 & GU,GG,GI & $34,35,36$ & 26 \\
\hline 9 & licoflavone $\mathrm{C}$ & Type II-1 & Acetone- $d_{6}$ & 400 & 13.00 & 161.0 & 99.3 & 107.4 & GU,GG,GI & $34,35,36$ & 36 \\
\hline 10 & lupiwighteone & Type II-1 & DMSO- $d_{6}$ & 400 & 12.90 & 159.6 & 98.8 & 104.0 & GU,GG,GI & $22,25,37$ & 10 \\
\hline 11 & isolupalbigenin & Type II-1 & DMSO- $d_{6}$ & 400 & 12.90 & 159.6 & 98.8 & 104.0 & GU & 10 & 10 \\
\hline 12 & 2,3-dehydrokievitone & Type II-1 & DMSO- $d_{6}$ & 400 & 12.92 & 159.9 & 98.8 & 104.9 & GG,GI & 38,26 & 26 \\
\hline 13 & licoisoflavone $\mathrm{C}$ & Type II-1 & DMSO- $d_{6}$ & 400 & 12.93 & 159.9 & 98.8 & 104.8 & GI & 26 & 26 \\
\hline 14 & gancaonin L & Type II-1 & DMSO- $d_{6}$ & 400 & 12.94 & 159.6 & 98.4 & 104.4 & GU,GG & 39,40 & 10 \\
\hline 15 & licoisoflavone B & Type II-2 & DMSO- $d_{6}$ & 400 & 12.85 & 161.8 & 98.9 & 104.6 & GU,GG,GI & $41,42,26$ & 10 \\
\hline 16 & licoisoflavone A & Type II-2 & DMSO- $d_{6}$ & 400 & 12.86 & 161.8 & 98.9 & 104.6 & GU,GG,GI & $41,35,26$ & 10 \\
\hline 17 & abiochanin A & Type II-2 & DMSO- $d_{6}$ & 400 & 12.91 & 161.9 & 99.3 & 104.0 & GU & 10 & 10 \\
\hline 18 & isoderrone & Type II-2 & DMSO- $d_{6}$ & 400 & 12.91 & 162.0 & 99.0 & 104.4 & GU,GI & 10,43 & 10 \\
\hline 19 & semilicoisoflavone B & Type II-2 & DMSO- $d_{6}$ & 400 & 12.94 & 162.4 & 99.4 & 104.8 & GU,GG,GI & $44,35,26$ & 26 \\
\hline 20 & apigenin & Type II-2 & DMSO- $d_{6}$ & 400 & 12.95 & 161.6 & 99.2 & 103.5 & GU & 45 & 46 \\
\hline 21 & genistein & Type II-2 & DMSO- $d_{6}$ & 400 & 12.95 & 162.0 & 99.0 & 104.5 & GU,GG,GI & $10,33,26$ & 10 \\
\hline 22 & 3'-isoprenylgenistein & Type II-2 & DMSO- $d_{6}$ & 400 & 12.95 & 162.4 & 99.3 & 104.9 & GU & 24 & 24 \\
\hline 23 & chrysoeriol & Type II-2 & DMSO- $d_{6}$ & 400 & 12.96 & 161.5 & 98.9 & 103.7 & GU & 47 & 48 \\
\hline 24 & pratensein & Type II-2 & DMSO- $d_{6}$ & 400 & 12.96 & 162.0 & 99.0 & 104.5 & GU & 10 & 10 \\
\hline 25 & luteolin & Type II-2 & DMSO- $d_{6}$ & 400 & 12.97 & 161.5 & 98.9 & 103.8 & GG & 49 & 50 \\
\hline 26 & epimedokoreanin D & Type II-2 & DMSO- $d_{6}$ & 400 & 12.98 & 161.5 & 98.8 & 103.7 & GU & 51 & 52 \\
\hline 27 & allolicoisoflavone B & Type II-2 & DMSO- $d_{6}$ & 400 & 12.98 & 161.9 & 99.2 & 103.9 & GU,GI & 53,26 & 10 \\
\hline 28 & glycyrrhisoflavone & Type II-2 & DMSO- $d_{6}$ & 400 & 13.00 & 162.5 & 99.4 & 104.9 & GU,GI & 45,26 & 26 \\
\hline 28 & glycyrrhisoflavone & Type II-2 & Acetone- $d_{6}$ & $\begin{array}{l}90 / 300 / \\
500\end{array}$ & 13.06 & 163.9 & 99.8 & 106.2 & GU,GI & 45,26 & 74 \\
\hline 29 & angustone $\mathrm{B}$ & Type II-3 & DMSO- $d_{6}$ & 80 & 13.07 & 158.8 & 111.3 & 104.6 & GG,GI & 42,41 & 14 \\
\hline 30 & angustone $\mathrm{A}$ & Type II-3 & DMSO- $d_{6}$ & 400 & 13.11 & 158.6 & 111.0 & 104.4 & GU,GI & 10,26 & 10 \\
\hline
\end{tabular}




\begin{tabular}{|c|c|c|c|c|c|c|c|c|c|c|c|}
\hline 31 & gancaonin A & Type II-3 & $\mathrm{DMSO}-d_{6}$ & 400 & 13.15 & 159.0 & 111.3 & 104.5 & GU & 22 & 22 \\
\hline 32 & gancaonin B & Type II-3 & DMSO- $d_{6}$ & 400 & 13.17 & 158.9 & 111.3 & 104.4 & GU & 22 & 22 \\
\hline 33 & gancaonin $\mathrm{O}$ & Type II-3 & DMSO- $d_{6}$ & 300 & 13.20 & 158.3 & 110.8 & 103.5 & GU & 39 & 54 \\
\hline 34 & gancaonin $\mathrm{H}$ & Type II-3 & $\mathrm{DMSO}-d_{6}$ & 400 & 13.22 & 158.9 & 111.2 & 104.3 & GU,GI & 24,41 & 12 \\
\hline 35 & wighteone & Type II-3 & $\mathrm{DMSO}-d_{6}$ & 400 & 13.23 & 158.8 & 111.0 & 104.2 & GU,GG,GI & $55,33,26$ & 10 \\
\hline 36 & 6- $C$-prenylorobol & Type II-3 & $\mathrm{DMSO}-d_{6}$ & 400 & 13.26 & 158.8 & 111.0 & 104.2 & GU & 10 & 10 \\
\hline 37 & $2^{\prime}, 4^{\prime}$-dihydroxy-chalcone & Type III-1 & $\mathrm{DMSO}-d_{6}$ & 500 & 13.40 & 165.8 & 102.6 & 113.0 & None & None & 56 \\
\hline 37 & $2^{\prime}, 4^{\prime}$-dihydroxy-chalcone & Type III-1 & $\mathrm{CDCl}_{3}$ & $\begin{array}{l}400 / 500 / \\
600\end{array}$ & 13.33 & 166.9 & 103.9 & 114.8 & None & None & 75 \\
\hline 38 & isoliquiritigenin & Type III-1 & $\mathrm{DMSO}-d_{6}$ & 400 & 13.58 & 165.8 & 102.6 & 113.0 & GU,GG,GI & $10,57,58$ & 10 \\
\hline 39 & licoagrochalcone A & Type III-1 & $\mathrm{DMSO}-d_{6}$ & 400 & 13.60 & 166.2 & 103.1 & 113.5 & GG,GI & 59,58 & 26 \\
\hline 39 & licoagrochalcone A & Type III-1 & Acetone- $d_{6}$ & 400 & 13.66 & 167.6 & 103.8 & 114.5 & GG,GI & 59,58 & 61 \\
\hline 40 & homobutein & Type III-1 & $\mathrm{DMSO}-d_{6}$ & 400 & 13.63 & 165.8 & 102.5 & 113.0 & GU & 10 & 10 \\
\hline 41 & isobavachalcone & Type III-2 & $\mathrm{DMSO}-d_{6}$ & 400 & 14.02 & 164.0 & 114.9 & 113.2 & GU,GG,GI & $60,61,26$ & 62 \\
\hline 41 & isobavachalcone & Type III-2 & $\mathrm{MeOH}-d_{4}$ & 200 & & 165.0 & 116.6 & 114.5 & GU,GG,GI & $60,61,26$ & 76 \\
\hline 42 & glabranin & Type I-1 & $\mathrm{CDCl}_{3}$ & 400 & 12.12 & 162.3 & 96.9 & 103.3 & GU,GG,GI & $63,20,17$ & 77 \\
\hline 43 & 8-prenyleriodictyol & Type I-1 & Acetone- $d_{6}$ & $400 / 500$ & 12.14 & 163.0 & 96.3 & 103.3 & GU,GI & 64,17 & 78 \\
\hline 44 & gancaonin $\mathrm{E}$ & Type I-1 & Acetone- $d_{6}$ & 400 & 12.14 & 163.0 & 96.4 & 103.4 & GU & 22 & 22 \\
\hline 45 & euchestraflavanone A & Type I-1 & $\mathrm{DMSO}-d_{6}$ & None & None & None & None & None & GI & 26 & None \\
\hline 46 & $\begin{array}{l}3^{\prime}-(\gamma, \gamma \text {-dimethylallyl }) \\
\text {-kievitone }\end{array}$ & Type I-1 & $\mathrm{DMSO}-d_{6}$ & None & None & None & None & None & GU & 65 & None \\
\hline 47 & dihydrolicoisoflavone & Type I-2 & Acetone- $d_{6}$ & 300 & & 165.4 & 96.6 & 102.8 & GU & 44 & 79 \\
\hline 48 & $\begin{array}{l}(2 S)-3^{\prime}-(2-\text { hydroxy-3-methylb } \\
\text { ut-3-enyl)-4',5,7-trihydroxy } \\
\text {-dihydroflavanone }\end{array}$ & Type I-2 & $\mathrm{DMSO}-d_{6}$ & None & None & None & None & None & GU & 28 & None \\
\hline 49 & glyasperin $\mathrm{F}$ & Type I-2 & $\mathrm{MeOH}-d_{4}$ & 500 & & 165.7 & 97.2 & 103.6 & GU & 44 & 80 \\
\hline 50 & isoglabranin & Type I-3 & $\mathrm{CDCl}_{3}$ & 400 & 12.36 & 161.2 & 107.0 & 102.9 & GG,GI & 25,17 & 81 \\
\hline 51 & 6-prenyleriodictyol & Type I-3 & $\mathrm{DMSO}-d_{6}$ & None & None & None & None & None & GU,GI & 66,17 & None \\
\hline 52 & paratocarpin L & Type I-3 & $\mathrm{DMSO}-d_{6}$ & None & None & None & None & None & GI & 41 & None \\
\hline 53 & glisoflavanone & Type I-3 & Acetone- $d_{6}$ & 500 & 12.31 & 162.7 & 109.2 & 102.6 & GU,GI & 53,26 & 53 \\
\hline 54 & gancaonin $\mathrm{M}$ & Type II-1 & Acetone- $d_{6}$ & 400 & 12.95 & 161.5 & 99.5 & 106.3 & GU & 39 & 39 \\
\hline 55 & vogelin $\mathrm{C}$ & Type II-1 & $\mathrm{DMSO}-d_{6}$ & None & None & None & None & None & GU & 55 & None \\
\hline 56 & glyurallin B & Type II-1 & $\mathrm{DMSO}-d_{6}$ & None & None & None & None & None & GU,GI & 31,41 & None \\
\hline 57 & orobol & Type II-2 & $\mathrm{DMSO}-d_{6}$ & None & None & None & None & None & GU & 24 & None \\
\hline 58 & allolicoisoflavone A & Type II-2 & $\mathrm{DMSO}-d_{6}$ & None & None & None & None & None & GU & 44 & None \\
\hline 59 & gancaonin Q & Type II-3 & Acetone- $d_{6}$ & 400 & 13.31 & 160.2 & 112.3 & 105.2 & GU,GI & 67,26 & 67 \\
\hline 60 & $6,5^{\prime}$-diprenylluteolin & Type II-3 & Acetone- $d_{6}$ & 500 & 13.31 & 160.2 & 112.3 & 105.3 & $\mathrm{GU}$ & 47 & 47 \\
\hline 61 & luteone & Type II-3 & $\mathrm{DMSO}-d_{6}$ & None & None & None & None & None & GU & 10 & None \\
\hline 62 & gancaonin $\mathrm{N}$ & Type II-3 & Acetone- $d_{6}$ & 400 & 13.03 & 160.4 & 112.3 & 105.9 & GU & 39 & 39 \\
\hline
\end{tabular}




\begin{tabular}{|c|c|c|c|c|c|c|c|c|c|c|c|}
\hline 63 & isoangustone $\mathrm{A}$ & Type II-3 & DMSO- $d_{6}$ & None & None & None & None & None & GU & 65 & None \\
\hline 64 & isochandalone & Type II-3 & DMSO- $d_{6}$ & None & None & None & None & None & GI & 26 & None \\
\hline 65 & 5-prenylbutein & Type III-1 & Acetone- $d_{6}$ & 500 & 13.68 & 167.4 & 103.6 & 114.6 & GI & 58 & 82 \\
\hline 66 & kanzonol B & Type III-1 & $\mathrm{DMSO}-d_{6}$ & None & None & None & None & None & GG,GI & 59,26 & None \\
\hline 67 & licochalcone $\mathrm{G}$ & Type III-1 & DMSO- $d_{6}$ & None & None & None & None & None & GI & 58 & None \\
\hline 68 & isoliquiritin & Type III-1 & $\mathrm{DMSO}-d_{6}$ & None & None & None & None & None & GU,GG & 68,69 & None \\
\hline 69 & isoliquiritin apioside & Type III-1 & DMSO- $d_{6}$ & None & None & None & None & None & GU,GG,GI & $68,69,70$ & None \\
\hline 70 & $6^{\prime \prime}-O$-acetylisoliquiritin & Type III-1 & DMSO- $d_{6}$ & None & None & None & None & None & GU & 71 & None \\
\hline 71 & $\begin{array}{l}\text { 1-(2,4-dihydroxyphenyl)-3-[4- } \\
\text { methoxy-3-(3-methylbut-2 } \\
\text {-enyl)-phenyl]prop-2-en-1-one }\end{array}$ & Type III-1 & DMSO- $d_{6}$ & None & None & None & None & None & GU & 60 & None \\
\hline 72 & morachalcone A & Type III-2 & Acetone- $d_{6}$ & 300 & & 164.5 & 115.9 & 114.1 & GG & 40 & 83 \\
\hline 73 & corylifol B & Type III-2 & DMSO- $d_{6}$ & None & None & None & None & None & GI & 41 & None \\
\hline 74 & kanzonol C & Type III-2 & $\begin{array}{l}\text { Acetone- } d_{6} \\
\mathrm{CDCl}_{3}\end{array}$ & $\begin{array}{l}400 \\
400\end{array}$ & $\begin{array}{l}14.02 \\
13.88\end{array}$ & None & None & None & GG,GI & $\begin{array}{l}72 \\
58\end{array}$ & $\begin{array}{l}84 \\
72\end{array}$ \\
\hline 75 & $\begin{array}{l}2,3^{\prime}, 4,4^{\prime} \text {-tetrahydroxy-3,5' } \\
\text {-diprenylchalcone }\end{array}$ & Type III-2 & DMSO- $d_{6}$ & None & None & None & None & None & GG & 40 & None \\
\hline 76 & $\begin{array}{l}6^{\prime \prime}, 6^{\prime \prime} \text {-dimethylpyrano[2' } 2^{\prime \prime}, 3^{\prime \prime}: 4, \\
5]-3^{\prime}-\gamma, \gamma \text {-dimethylally- } 2^{\prime}, 3,4^{\prime} \\
\text {-trihydroxychalcone }\end{array}$ & Type III-2 & DMSO- $d_{6}$ & None & None & None & None & None & GG & 38 & None \\
\hline
\end{tabular}


Table S4. The metabolomic HMBC patterns from experimental data of GU/GG/GI MeOH fractions (all in DMSO- $\left.d_{6}\right)^{a}{ }^{a}$

\begin{tabular}{|c|c|c|c|c|c|c|}
\hline $\begin{array}{l}\text { Structural } \\
\text { Type }\end{array}$ & $\begin{array}{l}\mathrm{MHz} \\
\left({ }^{1} \mathrm{H}\right)\end{array}$ & $\begin{array}{l}5-/ 2^{\prime}- \\
\mathrm{OH}\end{array}$ & $\begin{array}{l}\mathrm{C}-5^{b} / \\
\mathrm{C}-2^{\prime b}\end{array}$ & $\begin{array}{l}\mathrm{C}-6^{b} / \\
\mathrm{C}-3^{\prime b}\end{array}$ & $\begin{array}{l}\text { C- }-10^{b} / \\
\text { C- } 1^{b}\end{array}$ & $\begin{array}{l}\text { Plant/Fraction } \\
\text { Source }\end{array}$ \\
\hline Type I-1 & 600 & 12.11 & 160.8 & 95.4 & 101.7 & GI-MF \\
\hline Type I-1 & 600 & 12.27 & 161.5 & 95.5 & 102.1 & GU-MF \\
\hline Type I-2 & 600 & 12.12 & 163.6 & 95.9 & 101.8 & GI-MF \\
\hline Type I-2 & 600 & 12.13 & 163.6 & 95.9 & 101.8 & GI-MF \\
\hline Type I-2 & 600 & 12.14 & 163.6 & 95.9 & 101.9 & GU-MF \\
\hline Type I-2 & 600 & 12.14 & 163.6 & 95.9 & 101.8 & GI-MF \\
\hline Type I-2 & 600 & 12.15 & 163.6 & 95.9 & 101.8 & GI-MF \\
\hline Type I-2 & 600 & 12.15 & 163.7 & 95.9 & 101.9 & GG-MF \\
\hline Type I-2 & 600 & 12.15 & 163.6 & 95.9 & 101.9 & GU-MF \\
\hline Type I-2 & 600 & 12.15 & 163.6 & 95.9 & 101.8 & GI-MF \\
\hline Type I-2 & 600 & 12.16 & 163.8 & 95.9 & 101.9 & GG-MF \\
\hline Type I-2 & 600 & 12.29 & 163.9 & 95.9 & 102.1 & GU-MF \\
\hline Type I-2 & 600 & 12.31 & 163.9 & 95.9 & 102.2 & GU-MF \\
\hline Type I-2 & 600 & 12.32 & 163.9 & 95.9 & 102.2 & GU-MF \\
\hline Type I-3 & 600 & 12.41 & 160.7 & 107.5 & 101.6 & GU-MF \\
\hline Type I-3 & 600 & 12.42 & 160.5 & 107.6 & 101.5 & GU-MF \\
\hline Type I-3 & 600 & 12.57 & 161.0 & 107.7 & 102.0 & GU-MF \\
\hline Type I-3 & 600 & 12.59 & 161.0 & 107.6 & 102.1 & GU-MF \\
\hline Type II-1 & 600 & 12.80 & 159.4 & 98.4 & 104.5 & GU-MF \\
\hline Type II-1 & 600 & 12.90 & 159.1 & 98.4 & 103.7 & GI-MF \\
\hline Type II-1 & 600 & 12.91 & 159.5 & 98.5 & 104.5 & GU-MF \\
\hline Type II-1 & 600 & 12.91 & 159.1 & 98.4 & 103.7 & GI-MF \\
\hline Type II-1 & 600 & 12.92 & 159.6 & 98.6 & 104.6 & GU-MF \\
\hline Type II-2 & 600 & 12.86 & 161.9 & 99.0 & 104.7 & GU-MF \\
\hline Type II-2 & 600 & 12.92 & 162.0 & 99.1 & 104.5 & GU-MF \\
\hline Type II-2 & 600 & 12.96 & 162.1 & 99.1 & 104.5 & GU-MF \\
\hline Type II-2 & 600 & 12.96 & 162.0 & 99.0 & 104.4 & GU-MF \\
\hline Type II-2 & 600 & 12.97 & 161.4 & 98.9 & 103.6 & GI-MF \\
\hline Type II-2 & 600 & 12.97 & 162.0 & 99.0 & 104.4 & GU-MF \\
\hline Type II-2 & 600 & 12.97 & 161.4 & 98.9 & 103.6 & GI-MF \\
\hline Type II-2 & 600 & 12.98 & 161.4 & 99.0 & 103.5 & GI-MF \\
\hline Type II-2 & 600 & 13.01 & 162.0 & 99.0 & 104.5 & GU-MF \\
\hline Type II-3 & 600 & 13.12 & 158.8 & 111.0 & 104.5 & GU-MF \\
\hline Type II-3 & 600 & 13.12 & 158.8 & 111.0 & 104.5 & GU-MF \\
\hline Type II-3 & 600 & 13.22 & 158.8 & 111.1 & 104.4 & GU-MF \\
\hline Type II-3 & 600 & 13.22 & 158.3 & 111.1 & 103.6 & GI-MF \\
\hline Type II-3 & 600 & 13.22 & 158.8 & 111.1 & 104.4 & GU-MF \\
\hline Type II-3 & 600 & 13.23 & 158.8 & 111.1 & 104.3 & GU-MF \\
\hline Type II-3 & 600 & 13.24 & 158.8 & 111.1 & 104.3 & GU-MF \\
\hline Type II-3 & 600 & 13.25 & 158.8 & 111.1 & 104.3 & GU-MF \\
\hline Type II-3 & 600 & 13.28 & 158.8 & 111.0 & 104.3 & GU-MF \\
\hline Type III-1 & 600 & 13.54 & 165.8 & 102.6 & 113.0 & GU-MF \\
\hline Type III-1 & 600 & 13.54 & 165.8 & 102.6 & 113.0 & GI-MF \\
\hline Type III-1 & 600 & 13.54 & 165.8 & 102.6 & 113.0 & GG-MF \\
\hline Type III-1 & 600 & 13.62 & 165.8 & 102.6 & 113.0 & GU-MF \\
\hline Type III-1 & 600 & 13.62 & 165.8 & 102.6 & 113.0 & GG-MF \\
\hline Type III-1 & 600 & 13.62 & 165.8 & 102.6 & 113.0 & GI-MF \\
\hline Type III-1 & 600 & 13.64 & 165.8 & 102.6 & 112.9 & GI-MF \\
\hline Type III-2 & 600 & 13.96 & 163.7 & 115.0 & 112.4 & GG-MF \\
\hline Type III-2 & 600 & 14.01 & 163.6 & 114.9 & 112.3 & GG-MF \\
\hline Type III-2 & 600 & 14.02 & 163.7 & 114.9 & 112.3 & GG-MF \\
\hline Type III-2 & 600 & 14.04 & 163.7 & 115.0 & 112.3 & GG-MF \\
\hline
\end{tabular}


Table S5. The HMBC patterns of 15 compounds identified in GU-MF-11, GU-MF-12, and GU-MF-14-20 (all in DMSO- $d_{6}$ ).

\begin{tabular}{|c|c|c|c|c|c|c|c|c|}
\hline \multirow[t]{2}{*}{ No. } & \multirow{2}{*}{$\begin{array}{l}\text { Name Given } \\
\text { in the Literature }\end{array}$} & \multirow{2}{*}{$\begin{array}{l}\text { Structural } \\
\text { Type }\end{array}$} & \multirow{2}{*}{$\begin{array}{l}\mathrm{MHz} \\
\left({ }^{1} \mathrm{H}\right)\end{array}$} & \multicolumn{3}{|c|}{ Chemical Shift $^{a}$} & \multirow[b]{2}{*}{$\mathrm{C}-10^{b}$} & \multirow{2}{*}{$\begin{array}{l}\text { Fraction } \\
\text { Source }\end{array}$} \\
\hline & & & & $5-\mathrm{OH}$ & $\mathrm{C}-5^{b}$ & $\mathrm{C}-6^{b}$ & & \\
\hline 2 & 3'-( $\gamma, \gamma$-dimethylallyl)-kievitone & Type I-1 & 500 & 12.27 & 161.4 & 95.4 & 102.0 & GU-MF-15 \\
\hline 3 & licoisoflavanone & Type I-2 & 500 & 12.28 & 163.9 & 96.0 & 102.1 & GU-MF-11 \\
\hline 1 & 6-prenyl-licoisoflavanone & Type I-3 & 500 & 12.56 & 161.0 & 107.6 & 101.7 & GU-MF-18 \\
\hline 10 & lupiwighteone & Type II-1 & 500 & 12.90 & 159.6 & 98.5 & 104.5 & GU-MF-12 \\
\hline 11 & licoisoflavone A & Type II-2 & 500 & 12.84 & 162.0 & 98.9 & 104.7 & GU-MF-11 \\
\hline 12 & licoisoflavone B & Type II-2 & 500 & 12.85 & 161.8 & 98.9 & 104.6 & GU-MF-14 \\
\hline 15 & isoderrone & Type II-2 & 500 & 12.91 & 161.9 & 99.1 & 104.3 & GU-MF-15 \\
\hline 14 & semilicoisoflavone B & Type II-2 & 500 & 12.94 & 162.0 & 99.1 & 104.5 & GU-MF-11 \\
\hline 13 & glycyrrhisoflavone & Type II-2 & 500 & 12.99 & 162.0 & 99.0 & 104.6 & GU-MF-11 \\
\hline 4 & angustone $\mathrm{B}$ & Type II-3 & 500 & 13.10 & 158.6 & 111.0 & 104.2 & GU-MF-20 \\
\hline 7 & isochandalone & Type II-3 & 500 & 13.17 & 159.0 & 111.2 & 104.1 & GU-MF-20 \\
\hline 6 & gancaonin $\mathrm{H}$ & Type II-3 & 500 & 13.21 & 158.9 & 111.2 & 104.2 & GU-MF-17 \\
\hline 8 & wighteone & Type II-3 & 500 & 13.22 & 158.8 & 111.1 & 104.5 & GU-MF-12 \\
\hline 5 & isoangustone $\mathrm{A}$ & Type II-3 & 500 & 13.26 & 158.8 & 111.1 & 104.1 & GU-MF-16 \\
\hline 9 & 6,8-diprenylgenistein & none & 500 & 13.21 & 156.7 & 111.7 & 104.6 & GU-MF-19 \\
\hline
\end{tabular}


SECTION S5. REFERENCES RELATED TO STRUCTURE ELUCIDATION AND VERIFICATION

(1) Nam, J. W.; Phansalkar, R. S.; Lankin, D. C.; Bisson, J.; McAlpine, J. B.; Leme, A. A.; Vidal, C. M. P.; Ramirez, B.; Niemitz, M.; Bedran-Russo, A.; Chen, S. N.; Pauli, G. F. J. Org. Chem. 2015, 80, 7495-7507.

(2) Gao, W.; Napolitano, J. G.; Lankin, D. C.; Kim, J. Y.; Jin, Y. Y.; Lee, H.; Suh, J. W.; Chen, S. N.; Pauli, G. F. Magn. Reson. Chem. 2017, 55, 239-244.

(3) Napolitano, J. G.; Simmler, C.; McAlpine, J. B.; Lankin, D. C.; Chen, S. N.; Pauli, G. F. J. Nat. Prod. 2015, 78, 658-665.

(4) Napolitano, J. G.; Lankin, D. C.; McAlpine, J. B.; Niemitz, M.; Korhonen, S. P.; Chen, S.-N.; Pauli, G. F. J. Org. Chem. 2013, 78, 9963-9968.

(5) Napolitano, J. G.; Lankin, D. C.; Graf, T. N.; Friesen, J. B.; Chen, S. N.; McAlpine, J. B.; Oberlies, N. H.; Pauli, G. F. J. Org. Chem. 2013, 78, 2827-2839.

(6) Napolitano, J. G.; Lankin, D. C.; Chen, S. N.; Pauli, G. F. Magn. Reson. Chem. 2012, 50, 569-575.

(7) Napolitano, J. G.; Gödecke, T.; Rodríguez-Brasco, M. F.; Jaki, B. U.; Chen, S. N.; Lankin, D. C.; Pauli, G. F. J. Nat. Prod. 2012, 75, 238-248.

(8) Qiu, F.; Imai, A.; McAlpine, J. B.; Lankin, D. C.; Burton, I.; Karakach, T.; Farnsworth, N. R.; Chen, S. N.; Pauli, G. F. J. Nat. Prod. 2012, 75, 432-443.

(9) Dong, S. H.; Nikolić, D.; Simmler, C.; Qiu, F.; van Breemen, R. B.; Soejarto, D. D.; Pauli, G. F.; Chen, S. N. J. Nat. Prod. 2012, 75, 2168-2177.

(10) Ji, S.; Li, Z. W.; Song, W.; Wang, Y. R.; Liang, W. F.; Li, K.; Tang, S. N.; Wang, Q.; Qiao, X.; Zhou, D. M.; Yu, S. W.; Ye, M. J. Nat. Prod. 2016, 79, 281-292.

(11) Bojase, G.; Wanjala, C. C. W.; Majinda, R. R. T. Phytochemistry 2001, 56, 837-841.

(12) Fukai, T.; Wang, Q. H.; Kitagawa, T.; Kusano, K.; Nomura, T.; Litaka, Y. Heterocycles 1989, 29, 1761-1772.

(13) Sekine, T.; Inagaki, M.; Ikegami, F.; Fujii, Y.; Ruangrungsi, N. Phytochemistry 1999, 52, 87-94.

(14) Lane, G. A.; Newman, R. H. Phytochemistry 1987, 26, 295-300.

(15) Tahara, S.; Orihara, S.; Ingham, J. L.; Mizutani, J. Phytochemistry 1989, 28, 901-911.

(16) Hayashi, H.; Zhang, S. L.; Nakaizumi, T.; Shimura, K.; Yamaguchi, M.; Inoue, K.; Sarsenbaev, K.; Ito, M.; Honda, G. Chem. Pharm. Bull. 2003, 51, 1147-1152.

(17) Zhou, B.; Wan, C. X. J. Asian Nat. Prod. Res. 2015, 17, 256-261.

(18) Stevens, J. F.; Ivancic, M.; Hsu, V. L.; Deinzer, M. L. Phytochemistry 1997, 44, 15751585.

(19) Zhu, X. M.; Di, Y. T.; Peng, S. L.; Wang, M. K.; Ding, L. S. Chin. Tradit. Herb. Drugs 2003, 34, 198-201.

(20) Biondi, D. M.; Rocco, C.; Ruberto, G. J. Nat. Prod. 2003, 66, 477-480.

(21) An, N.; Yang, S. L.; Zou, Z. M.; Xu, L. Z. Chin. Tradit. Herb. Drugs 2006, 37, 663-664.

(22) Fukai, T.; Wang, Q. H.; Nomura, T. Heterocycles 1989, 29, 1369-1378.

(23) Ndom, J. C.; Mbafor, J. T.; Fomum, Z. T.; Martin, M. T.; Bodo, B. Magn. Reson. Chem. 1993, 31, 210-211.

(24) Wang, Q.; Miao, W. J.; Xiang, C.; Guo, D. A.; Ye, M. Chin. Tradit. Herb. Drugs 2014, 45, 31-36.

(25) Biondi, D. M.; Rocco, C.; Ruberto, G. J. Nat. Prod. 2005, 68, 1099-1102.

(26) Lin, Y.; Kuang, Y.; Li, K.; Wang, S.; Ji, S.; Chen, K.; Song, W.; Qiao, X.; Ye, M. Bioorg. Med. Chem. 2017, 25, 5522-5530. 
(27) Jeon, S. H.; Chun, W.; Choi, Y. J.; Kwon, Y. S. Arch. Pharm. Res. 2008, 31, 978-982.

(28) Zhou, B.; Wan, C. X. Chin. Tradit. Herb. Drugs 2016, 47, 21-25.

(29) Fukui, H.; Goto, K.; Tabata, M. Chem. Pharm. Bull. 1988, 36, 4174-4176.

(30) Nkengfack, A. E.; Sanson, D. R.; Tempesta, M. S.; Fomum, Z. T. J. Nat. Prod. 1989, 52, 320-324.

(31) Shibano, M.; Henmi, A.; Matsumoto, Y.; Kusano, G.; Miyase, T.; Hatakeyama, Y. Heterocycles 1997, 45, 2053-2060.

(32) Montoro, P.; Maldini, M.; Russo, M.; Postorino, S.; Piacente, S.; Pizza, C. J. Pharm. Biomed. Anal. 2011, 54, 535-544.

(33) Hayashi, H.; Yasuma, M.; Hiraoka, N.; Ikeshiro, Y.; Yamamoto, H.; Yesilada, E.; Sezik, E.; Honda, G.; Tabata, M. Phytochemistry 1996, 42, 701-704.

(34) Guo, Z. H.; Niu, X. L.; Xiao, T.; Lu, J. J.; Li, W.; Zhao, Y. Q. J. Funct. Foods 2015, 14, 324-336.

(35) Fang, S. Q.; Leng, K.; Duan, J. A.; Li, C. Y.; Wei, J. H.; Zheng, Y. F.; Peng, G. P. Chin. Tradit. Pat. Med. 2015, 37, 2443-2448.

(36) Kajiyama, K.; Demizu, S.; Hiraga, Y.; Kinoshita, K.; Koyama, K.; Takahashi, K.; Tamura, Y.; Okada, K.; Kinoshita, T. J. Nat. Prod. 1992, 55, 1197-1203.

(37) Lin, Y.; Kuang, Y.; Li, K.; Wang, S.; Song, W.; Qiao, X.; Sabir, G.; Ye, M. Bioorg. Med. Chem. 2017, 25, 3706-3713.

(38) Li, K.; Ji, S.; Song, W.; Kuang, Y.; Lin, Y.; Tang, S. N.; Cui, Z. X.; Qiao, X.; Yu, S. W.; Ye, M. J. Nat. Prod. 2017, 80, 334-346.

(39) Fukai, T.; Wang, Q. H.; Takayama, M.; Nomura, T. Heterocycles 1990, 31, 373-382.

(40) Kuroda, M.; Mimaki, Y.; Honda, S.; Tanaka, H.; Yokota, S.; Mae, T. Bioorg. Med. Chem. 2010, 18, 962-970.

(41) Song, W.; Qiao, X.; Chen, K.; Wang, Y.; Ji, S.; Feng, J.; Li, K.; Lin, Y.; Ye, M. Anal. Chem. 2017, 89, 3146-3153.

(42) Li, Y. J.; Chen, J.; Li, Y.; Li, Q.; Zheng, Y. F.; Fu, Y.; Li, P. J. Chromatogr. A 2011, 1218, 8181-8191.

(43) Fukai, T.; Nomura, T. Phytochemistry 1995, 38, 759-765.

(44) Gafner, S.; Bergeron, C.; Villinski, J. R.; Godejohann, M.; Kessler, P.; Cardellina, J. H.; Ferreira, D.; Feghali, K.; Grenier, D. J. Nat. Prod. 2011, 74, 2514-2519.

(45) Li, S. P.; Li, W.; Wang, Y. H.; Asada, Y.; Koike, K. Bioorg. Med. Chem. Lett. 2010, 20, 5398-5401.

(46) Li, W.; Dai, R. J.; Yu, Y. H.; Li, L.; Wu, C. M.; Luan, W. W.; Meng, W. W.; Zhang, X. S.; Deng, Y. L. Biol. Pharm. Bull. 2007, 30, 1123-1129.

(47) Bai, H.; Li, W.; Koike, K.; Pei, Y. P.; Dou, D. Q.; Chen, Y. J.; Wang, Y. H.; Nikaido, T. Heterocycles 2004, 63, 2091-2095.

(48) Park, Y.; Moon, B. H.; Yang, H.; Lee, Y.; Lee, E.; Lim, Y. Magn. Reson. Chem. 2007, 45, 1072-1075.

(49) Khalaf, I.; Vlase, L.; Lazar, D.; Corciova, A.; Ivanescu, B.; Lazar, M. I. Farmacia 2010, 58, 416-421.

(50) Park, Y.; Moon, B. H.; Lee, E.; Lee, Y.; Yoon, Y.; Ahn, J. H.; Lim, Y. Magn. Reson. Chem. 2007, 45, 674-679.

(51) Fang, S. Q.; Qu, Q. Y.; Zheng, Y. F.; Zhong, H. H.; Shan, C. X.; Wang, F.; Li, C. Y.; Peng, G. P. J. Sep. Sci. 2016, 39, 2068-2078.

(52) Li, W. K.; Pan, J. Q.; Lü, M. J.; Zhang, R. Y.; Liao M. C.; Xiao, P. G. Acta Pharmaceutica 
Sinica 1996, 31, 29-32.

(53) Hatano, T.; Aga, Y.; Shintani, Y.; Ito, H.; Okuda, T.; Yoshida, T. Phytochemistry 2000, 55, 959-963.

(54) Dias, A. C. P.; Tomás-Barberán, F. A.; Fernandes-Ferreira, M.; Ferreres, F. Phytochemistry 1998, 48, 1165-1168.

(55) Luo, L. P.; Shen, L. M.; Sun, F.; Ma, Z. J. Food Chem. 2013, 138, 315-320.

(56) Zhang, Y. L.; Mei, R. Q.; Liu, X.; Liu, G. M.; Wu, B. Chin. Tradit. Herb. Drugs 2014, 45, 2293-2298.

(57) Chin, Y. W.; Jung, H. A.; Liu, Y.; Su, B. N.; Castoro, J. A.; Keller, W. J.; Pereira, M. A.; Kinghorn, A. D. J. Agric. Food Chem. 2007, 55, 4691-4697.

(58) Dao, T. T.; Nguyen, P. H.; Lee, H. S.; Kim, E.; Park, J.; Lim, S. I.; Oh, W. K. Bioorg. Med. Chem. Lett. 2011, 21, 294-298.

(59) Asada, Y.; Li, W.; Yoshikawa, T. Phytochemistry 1998, 47, 389-392.

(60) Feng, K. P.; Chen, R. D.; Li, J. H.; Tao, X. Y.; Liu, J. M.; Zhang, M.; Dai, J. G. J. Asian Nat. Prod. Res. 2016, 18, 253-259.

(61) Asada, Y.; Li, W.; Yoshikawa, T. Phytochemistry 1998, 47, 389-392.

(62) Wang, H. M.; Yan, Z. H.; Lei, Y. N.; Sheng, K.; Yao, Q. W.; Lu, K.; Yu, P. Tetrahedron Lett. 2014, 55, 897-899.

(63) Yuldashev, M. P. Chem. Nat. Compd. 1998, 34, 508-509.

(64) Jia, S. S.; Liu, D.; Wang, H. Q.; Suo, Z. X. Acta Pharmaceutica Sinica 1993, 28, 623-625.

(65) Eerdunbayaer; Orabi, M. A. A.; Aoyama, H.; Kuroda, T.; Hatano, T. Molecules 2014, 19, 13027-13041.

(66) Ye, R. G.; Fan, Y. H.; Ma, C. M. J. Agric. Food Chem. 2017, 65, 510-515.

(67) Fukai, T.; Wang, Q. H.; Nomura, T. Phytochemistry 1991, 30, 1245-1250.

(68) Kitagawa, I.; Hori, K.; Uchida, E.; Chen, W. Z.; Yoshikawa, M.; Ren, J. L. Chem. Pharm. Bull. 1993, 41, 1567-1572.

(69) Kitagawa, I.; Chen, W. Z.; Hori, K.; Harada, E.; Yasuda, N.; Yoshikawa, M.; Ren, J. L. Chem. Pharm. Bull. 1994, 42, 1056-1062.

(70) Li, G. N.; Simmler, C.; Chen, L. Y.; Nikolic, D.; Chen, S. N.; Pauli, G. F.; van Breemen, R. B. Eur. J. Pharm. Sci. 2017, 109, 182-190.

(71) Lee, J. E.; Lee, J. Y.; Kim, J.; Lee, K.; Choi, S. U.; Ryu, S. Y. Arch. Pharm. Res. 2015, 38, 1299-1303.

(72) Kinoshita, T.; Kajiyama, K.; Hiraga, Y.; Takahashi, K.; Tamura, Y.; Mizutani, K. Chem. Pharm. Bull. 1996, 44, 1218-1221.

(73) Shirataki, Y.; Noguchi, M.; Yokoe, I.; Tomimori, T.; Komatsu, M. Chem. Pharm. Bull. 1991, 39, 1568-1572.

(74) Hatano, T.; Kagawa, H.; Yasuhara, T.; Okuda, T. Chem. Pharm. Bull. 1988, 36, 2090-2097.

(75) Zhang, X. J.; Li, L. Y.; Wang, S. S.; Que, S.; Yang, W. Z.; Zhang, F. Y.; Gong, N. B.; Cheng, W.; Liang, H.; Ye, M.; Jia, Y. X.; Zhang, Q. Y. Tetrahedron 2013, 69, 11074-11079.

(76) Pistelli, L.; Spera, K.; Flamini, G.; Mele, S.; Morelli, I. Phytochemistry 1996, 42, 14551458.

(77) Peralta, M. A.; Santi, M. D.; Agnese, A. M.; Cabrera, J. L.; Ortega, M. G. Phytochemistry lett. 2014, 10, 260-267.

(78) Fukai, T.; Zeng, L.; Nomura, T.; Zhang, R. Y.; Lou, Z. C. Nat. Medicines 1996, 50, 247251.

(79) Dubois, J. L.; Sneden, A. T. J. Nat. Prod. 1995, 58, 629-632. 
(80) McKee, T. C.; Bokesch, H. R.; McCormick, J. L.; Rashid, M. A.; Spielvogel, D.; Gustafson, K. R.; Alavanja, M. M.; Cardellina, J. H.; Boyd, M. R. J. Nat. Prod. 1997, 60, 431-438.

(81) Popoola, O. K.; Marnewick, J. L.; Rautenbach, F.; Ameer, F.; Iwuoha, E. I.; Hussein, A. A. Molecules 2015, 20, 7143-7155.

(82) Yenesew, A.; Induli, M.; Derese, S.; Midiwo, J. O.; Heydenreich, M.; Peter, M. G.; Akala, H.; Wangui, J.; Liyala, P.; Waters, N. C. Phytochemistry 2004, 65, 3029-3032.

(83) Monache, G. D.; Rosa, M. C. D.; Scurria, R.; Vitali, A.; Cuteri, A.; Monacelli, B.; Pasqua, G.; Botta, B. Phytochemistry 1995, 39, 575-580.

(84) Fukai, T.; Nishizawa, J.; Nomura, T. Phytochemistry 1994, 35, 515-519. 
SECTION S6. FURTHER SUPPORTING FIGURES \& TABLES

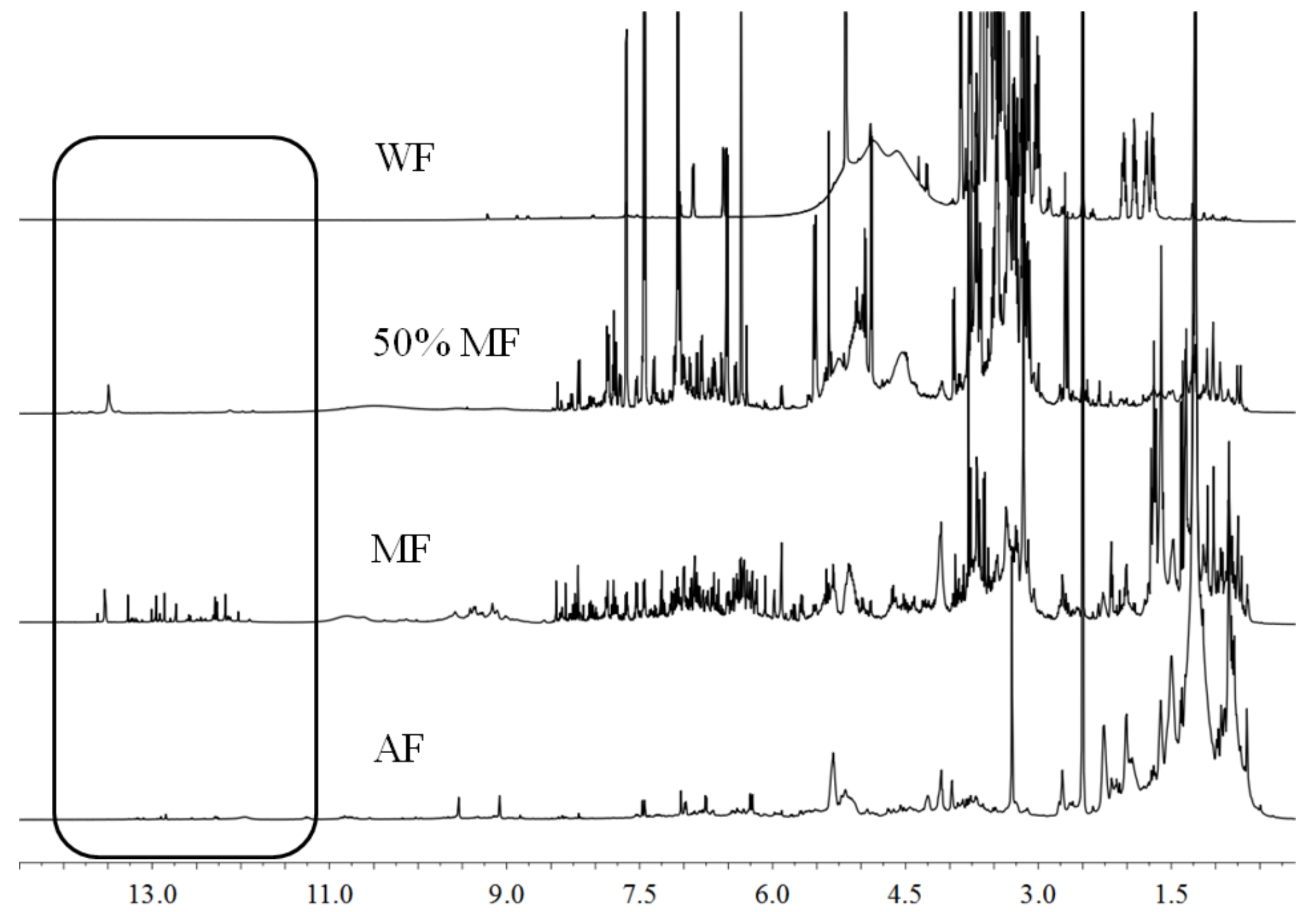

Figure S5. Comparison of the ${ }^{1} \mathrm{H}$ NMR spectra of WF, $50 \% \mathrm{MF}, \mathrm{MF}$, and AF for GU in DMSO- $d_{6}$. 


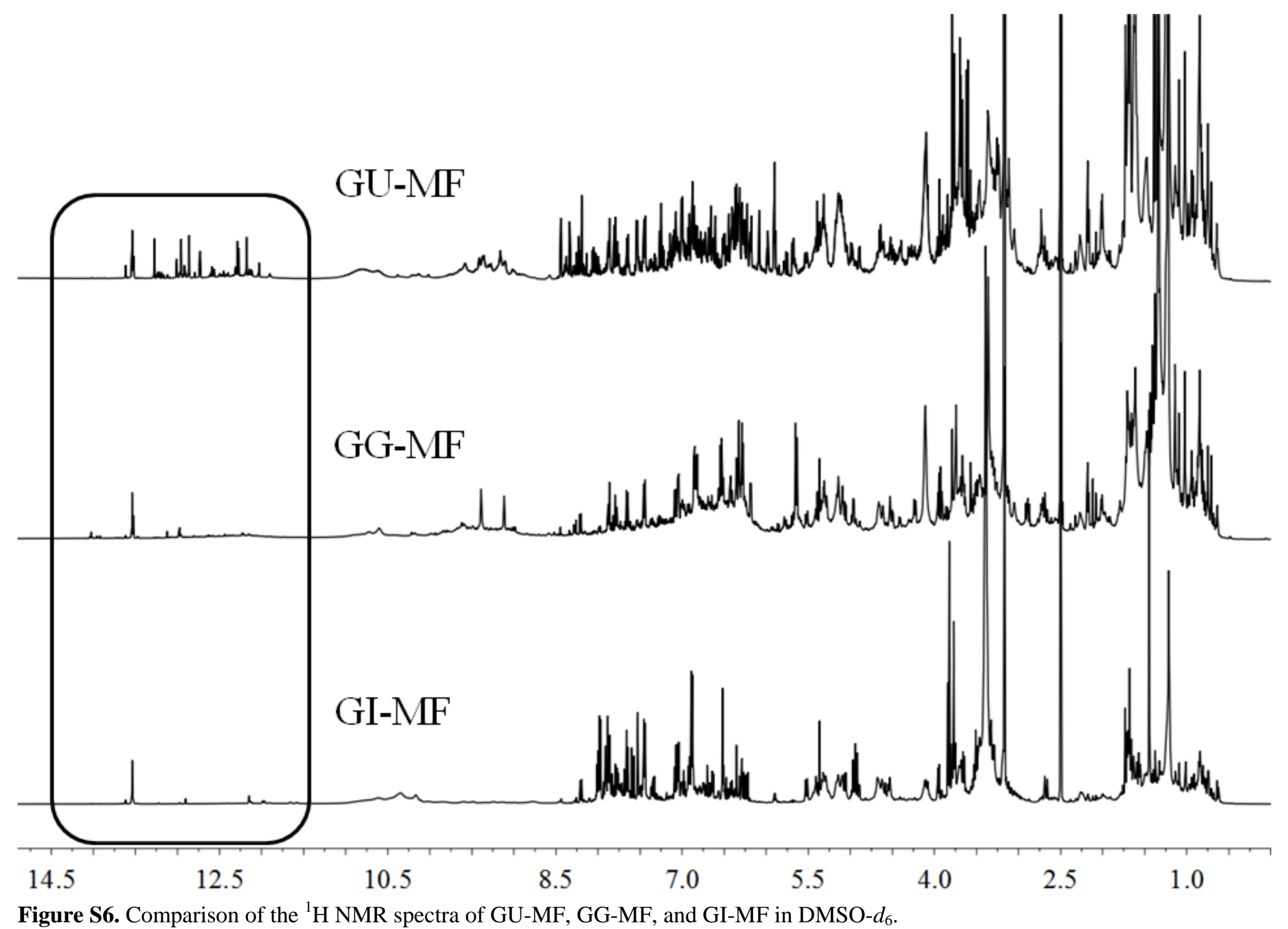


<smiles>O=C1CC(c2ccc(O)cc2)Oc2cc(O)cc(O)c21</smiles><smiles>O=c1cc(-c2ccc(O)cc2)oc2cc(O)cc(O)c12</smiles>

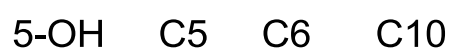
$\begin{array}{lll}12.950 & 161.6 \quad 99.2 & 103.5\end{array}$

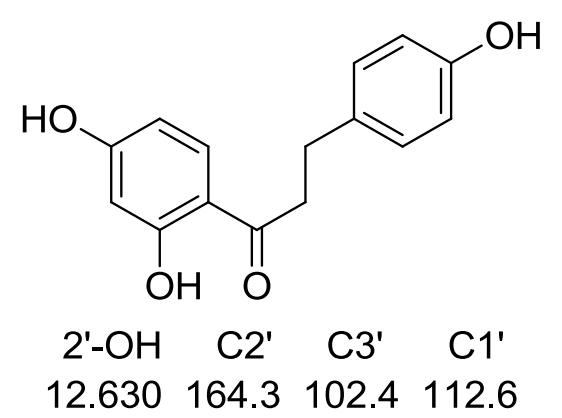

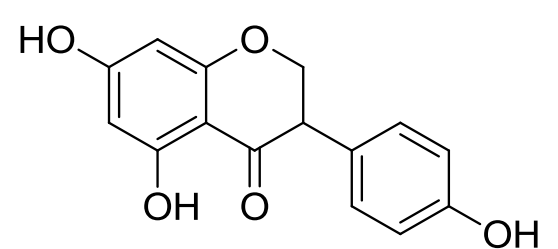

$5-\mathrm{OH} \quad \mathrm{C} 5 \quad \mathrm{C} 6 \quad \mathrm{C} 10$ $12.180163 .8 \quad 95.8 \quad 101.6$<smiles>O=c1c(-c2ccc(O)cc2)coc2cc(O)cc(O)c12</smiles>

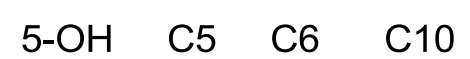
$12.950 \quad 162.0 \quad 99.0 \quad 104.5$<smiles>O=C(/C=C/c1ccc(O)cc1)c1ccc(O)cc1O</smiles>

$$
\text { 2'-OH C2' C3' C1' }
$$$$
13.580165 .0102 .6113 .0
$$<smiles>O=C1c2c(O)cc(O)cc2OC(c2ccc(O)cc2)C1O</smiles>

5-OH C5 C6 C10

11.920163 .596 .2100 .6<smiles>[R]c1c(-c2ccc(O)cc2)oc2cc(O)cc(O)c2c1=O</smiles>

5-OH C5 C6 C10

$\mathrm{R}=\mathrm{H} \quad \begin{array}{lllll}12.480 & 160.7 & 98.2 & 103.0\end{array}$

$\mathrm{R}=\mathrm{CH}_{3} \quad 12.700 \quad 161.298 .5 \quad 104.1$

$\mathrm{R}=\mathrm{Glc} \quad 12.600 \quad 161.2 \quad 98.7 \quad 104.0$

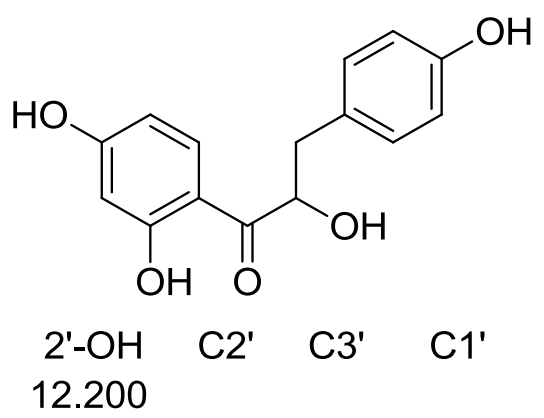

Figure S7. The chemical shifts of the 5-/2'-OH hydrogens and the three HMBC correlated carbons of the following flavonoid skeletons: flavonone, isoflavonone, flavononol, flavone, isoflavone, flavonol, its 3-O-methyl derivative, its 3-O-glycoside, dihydrochalcone, chalcone, and $\alpha$-hydroxyl dihydrochalcone (all in DMSO- $d_{6}$ ). 


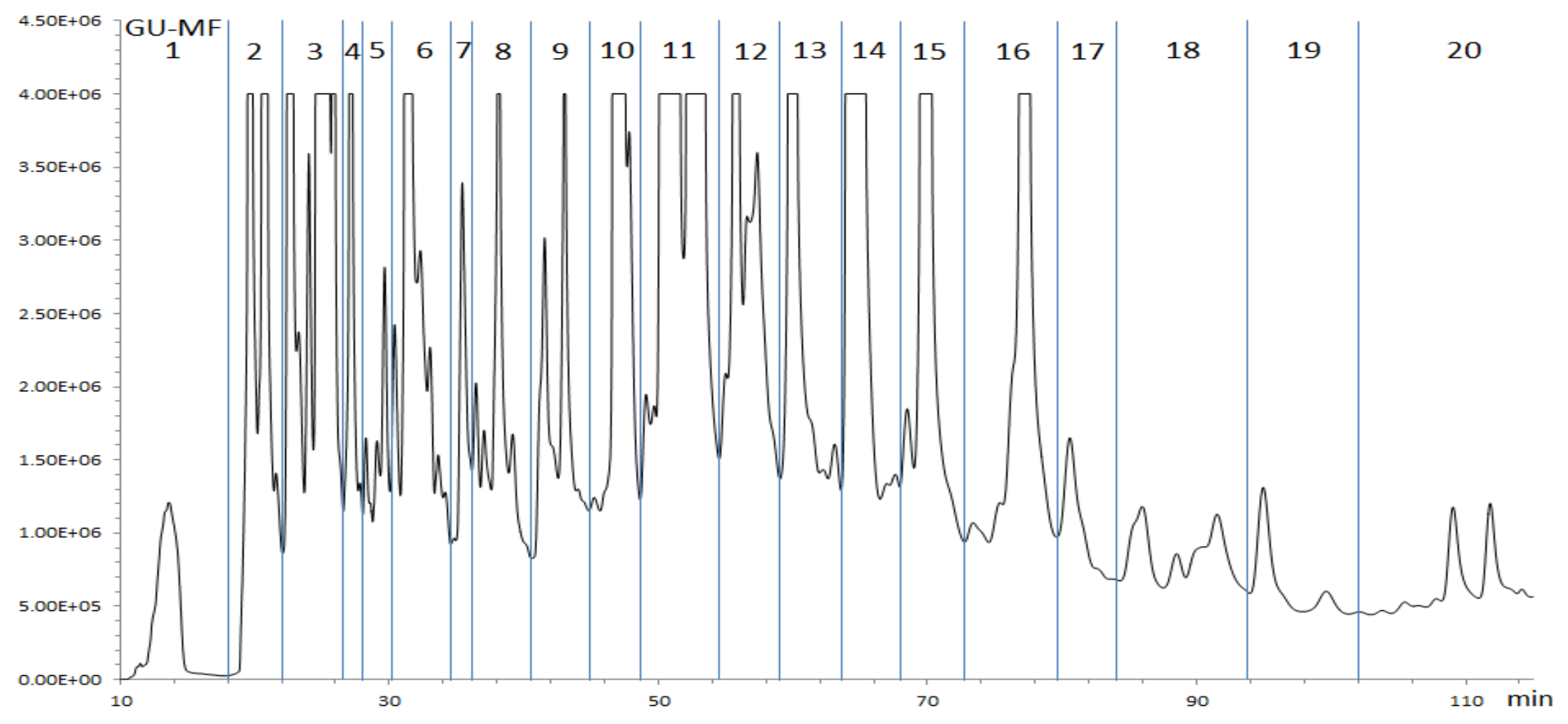

Figure S8. The preparative HPLC profile of GU-MF at 254 nm, divided into 20 subfractions, GU-MF-1 to GU-MF-20. 

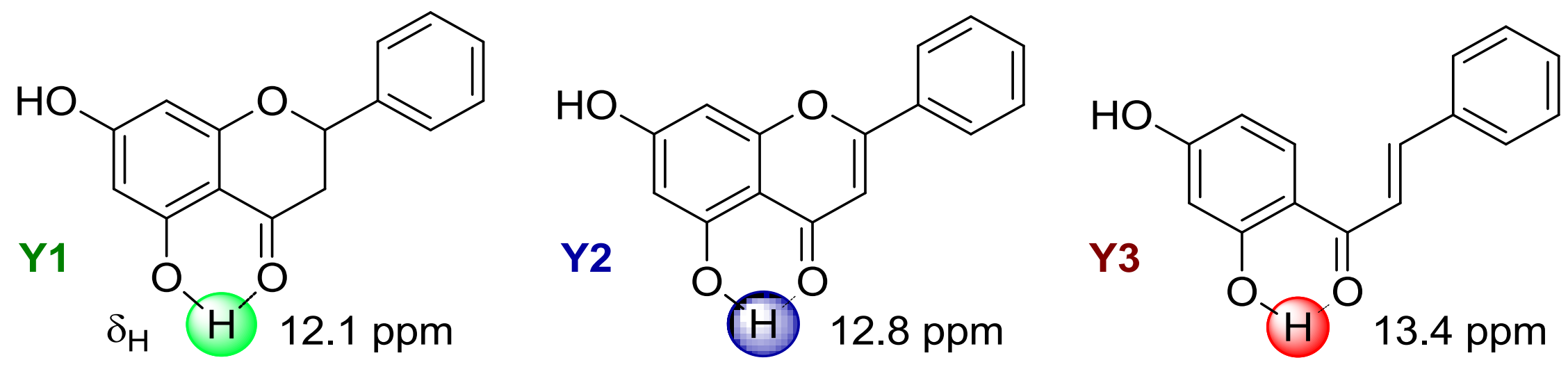

Figure S9. The 5-/2'-OH hydrogen chemical shifts and structures of the three model compounds, Y1, Y2, and Y3 in DMSO- $d_{6}$. In order to theoretically explain the above rules of 5-OH hydrogen chemical shifts for flavanones, flavonoids, and chalcones, three model compounds, Y1, Y2, and Y3, were chosen and further studied by using Natural Bond Orbital (NBO) analysis. Firstly, conformational searching were carried out via Monte Carlo searching using molecular mechanism with MMFF94 force field in the Spartan 08 program. ${ }^{1}$ The results showed two lowest energy conformers for each compound (Fig. S10). Subsequently, the conformers were re-optimized using DFT at the B3LYP/6-31+G(d,p) level in vacuum with the Gaussian 09 program. ${ }^{2}$ The B3LYP/6-31+G(d,p) harmonic vibrational frequencies were further calculated to confirm their stability. The second-order perturbation stabilization energies $\Delta E^{2}$ (an indicator of intensity of the $4=\mathrm{O} \ldots \mathrm{H}-\mathrm{O}-5$ or $1=\mathrm{O} \ldots \mathrm{H}-\mathrm{O}-2{ }^{\prime}$ hydrogen bond $)^{3}$ and natural charges on the three related atoms were calculated on each conformer via Natural Bond Orbital (NBO) analysis at the WB97XD/6-311++G(2d,2p) level. The final data for the compounds were obtained by averaging those of the conformers according to their relative Gibbs free energy $(\Delta \mathrm{G})$ and Boltzmann distribution theory. 
Table S6. Bond lengths, second-order stabilization energies $\Delta E^{(2)}$, and natural charges of the hydrogen atoms of the $\mathrm{C}(4 / 1)=\mathrm{O} \ldots \mathrm{H}-\mathrm{O}-\mathrm{C}\left(5 / 2^{\prime}\right)$ hydrogen bonds in the model compounds $\mathrm{Y} 1$, Y2, and $\mathrm{Y} 3$.

\begin{tabular}{lllll}
\hline Compound & Donor $\rightarrow$ Acceptor & $\mathrm{R}(\mathrm{O} \ldots \mathrm{H})(\mathrm{nm})$ & $\Delta E^{(2)}(\mathrm{Kcal} / \mathrm{mol})$ & Natural Charge \\
\hline $\mathrm{Y} 1$ & $\mathrm{E}(\mathrm{LP}(\mathrm{O}=\mathrm{C}(4)) \rightarrow \mathrm{RY} *(\mathrm{H}-\mathrm{O}-\mathrm{C}(5))$ & 0.170428 & 0.56 & 0.51274 \\
$\mathrm{Y} 2$ & $\mathrm{E}\left(\mathrm{LP}(\mathrm{O}=\mathrm{C}(4)) \rightarrow \mathrm{RY}{ }^{*}(\mathrm{H}-\mathrm{O}-\mathrm{C}(5))\right.$ & 0.168642 & 0.58 & 0.51489 \\
$\mathrm{Y} 3$ & $\mathrm{E}\left(\mathrm{LP}(\mathrm{O}=\mathrm{C}(4 / 1)) \rightarrow \mathrm{RY}^{*}\left(\mathrm{H}-\mathrm{O}-\mathrm{C}\left(5 / 2^{\prime}\right)\right)\right.$ & 0.159827 & 0.73 & 0.51162 \\
\hline
\end{tabular}

The results showed a decrease of hydrogen bond lengths and increase of second-order stabilization energies from Y1 to Y3, which is consistent with the increase of 5-/2'-OH proton chemical shifts of these compounds and indicating the hydrogen bonds play important roles in the NMR behavior of the 5-/2'-OH protons. However, the electron density on the hydrogen atom of the 5-/2'-OH plays less important role in the determination of the chemical shifts because the order of natural charges on these hydrogen atoms are inconsistent with the NMR observation.

\section{Methods \& References}

1) Spartan 08; Wavefunction Inc.:Irvine, CA.

2) Gaussian 09, Revision A.1, Frisch, M. J.; Trucks, G. W.; Schlegel, H. B.; Scuseria, G. E.; Robb, M. A.; Cheeseman, J. R.; Scalmani, G.; Barone, V.; Mennucci, B.; Petersson, G. A.; Nakatsuji, H.; Caricato, M.; Li, X.; Hratchian, H. P.; Izmaylov, A. F.; Bloino, J.; Zheng, G.; Sonnenberg, J. L.; Hada, M.; Ehara, M.; Toyota, K.; Fukuda, R.; Hasegawa, J.; Ishida, M.; Nakajima, T.; Honda, Y.; Kitao, O.; Nakai, H.; Vreven, T.; Montgomery, Jr., J. A.; Peralta, J. E.; Ogliaro, F.; Bearpark, M.; Heyd, J. J.; Brothers, E.; Kudin, K. N.; Staroverov, V. N.; Kobayashi, R.; Normand, J.; Raghavachari, K.; Rendell, A.; Burant, J. C.; Iyengar, S. S.; Tomasi, J.; Cossi, M.; Rega, N.; Millam, J. M.; Klene, M.; Knox, J. E.; Cross, J. B.; Bakken, V.; Adamo, C.; Jaramillo, J.; Gomperts, R.; Stratmann, R. E.; Yazyev, O.; Austin, A. J.; Cammi, R.; Pomelli, C.; Ochterski, J. W.; Martin, R. L.; Morokuma, K.; Zakrzewski, V. G.; Voth, G. A.; Salvador, P.; Dannenberg, J. J.; Dapprich, S.; Daniels, A. D.; Farkas, Ö.; Foresman, J. B.; Ortiz, J. V.; Cioslowski, J.; Fox, D. J. Gaussian, Inc., Wallingford CT, 2009.

3) J. Phys. Chem. B 2008, 112, 5088-5097. 


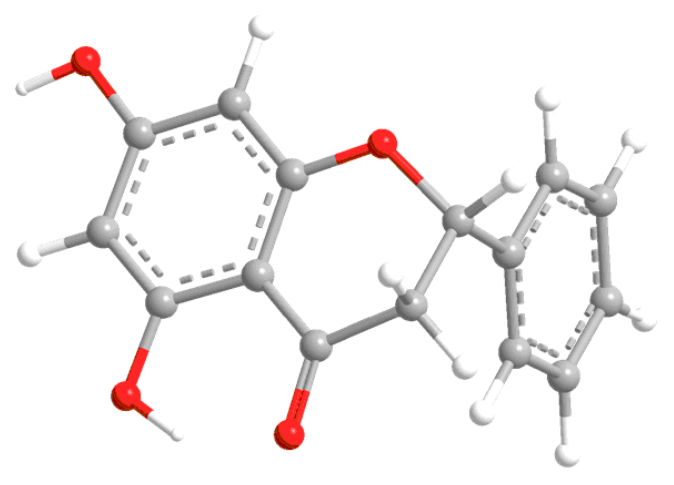

Y1C1

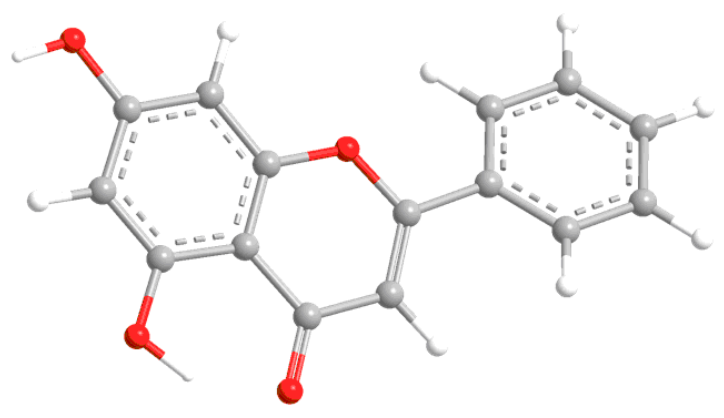

Y2C1

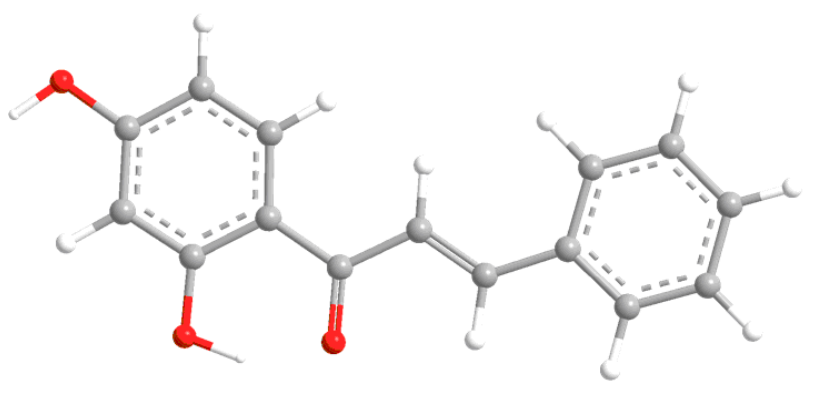

Y 3C1

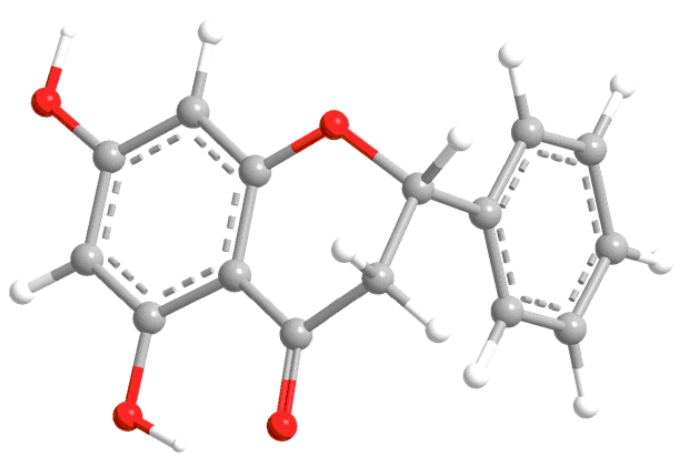

Y1C2

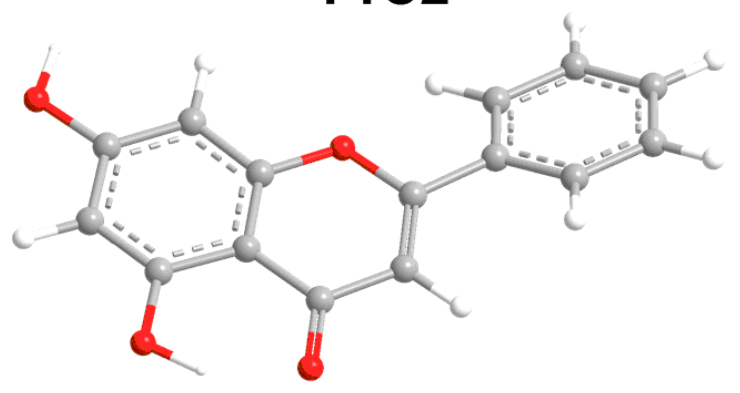

Y2C2

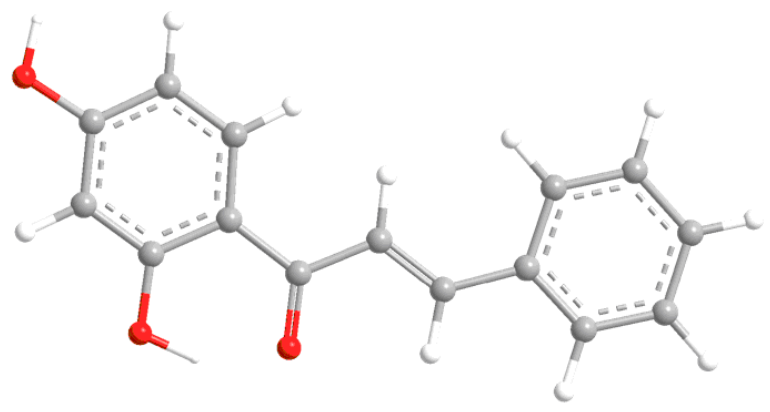

Y3C2

Figure S10. B3LYP/6-31+G(d,p) optimized lowest energy 3D conformers of the model compounds, Y1, Y2, and Y3. In order to obtain the final spectra, all simulated spectra of the conformers of each compound were averaged according to their energies and the Boltzmann distribution theory expressed as:

$$
\frac{N_{i}^{*}}{N}=\frac{g_{i} e^{-\varepsilon_{i} / k_{B} T}}{\sum g_{i} e^{-\varepsilon_{i} / k_{B} T}}
$$


Table S7. Energy analysis of the conformers of the model compounds, Y1, Y2, and Y3.

\begin{tabular}{llll}
\hline conf. & \multicolumn{4}{l}{ Gibbs free energy $(298.15 \mathrm{~K})$} \\
\hline & $\mathrm{G}($ Hartree $)$ & $\Delta \mathrm{G}(\mathrm{Kcal} / \mathrm{mol})$ & Boltzmann Distribution \\
$\mathrm{Y} 1 \mathrm{C} 1$ & -879.586629 & 0 & 0.618004 \\
$\mathrm{Y} 1 \mathrm{C} 2$ & -879.586175 & 0.284890 & 0.381996 \\
$\mathrm{Y} 2 \mathrm{C} 1$ & -878.406073 & 0 & 0.730286 \\
$\mathrm{Y} 2 \mathrm{C} 2$ & -878.405133 & 0.589859 & 0.269714 \\
$\mathrm{Y} 3 \mathrm{C} 1$ & -804.361161 & 0 & 0.677843 \\
$\mathrm{Y} 3 \mathrm{C} 2$ & -804.360459 & 0.440512 & 0.322157 \\
\hline
\end{tabular}

Table S8. NBO analysis data for conformers of the model compounds, Y1, Y2, and Y3: natural charges on hydrogen bond related atoms.

\begin{tabular}{llll}
\hline Y1C1 & Natural Charge & Y1C2 & Natural Charge \\
\hline $4=\mathrm{O}$ & -0.6452 & $4=\mathrm{O}$ & -0.64405 \\
$5-\mathrm{O}$ & -0.68451 & $5-\mathrm{O}$ & -0.68231 \\
$5-\mathrm{O}-\mathrm{H}$ & 0.51312 & $5-\mathrm{O}-\mathrm{H}$ & 0.51212 \\
\hline \multicolumn{5}{r}{} \\
\hline Y2C1 & Natural Charge & Y2C2 & Natural Charge \\
\hline 4=O & -0.66364 & $4=\mathrm{O}$ & -0.66269 \\
$5-\mathrm{O}$ & -0.68754 & $5-\mathrm{O}$ & -0.68577 \\
$5-\mathrm{O}-\mathrm{H}$ & 0.51521 & $5-\mathrm{O}-\mathrm{H}$ & 0.51402 \\
\hline \multicolumn{5}{r}{} & & \\
\hline Y3C1 & Natural Charge & Y3C2 & Natural Charge \\
\hline 1=O & -0.65874 & 1=O & -0.65739 \\
2'-O & -0.68947 & 2'-O & -0.68688 \\
2'-O-H & 0.51182 & 2'-O-H & 0.51121 \\
\hline
\end{tabular}


Table S9. Second-order stabilization energies $\Delta E^{(2)}(\mathrm{kcal} / \mathrm{mol})$ of the hydrogen bonds of the conformers of the model compounds, Y1, Y2, and Y3.

\begin{tabular}{ll}
\hline & $\Delta E^{(2)}(\mathrm{kcal} / \mathrm{mol})$ \\
\hline $\mathrm{Y} 1 \mathrm{C} 1$ & 0.56 \\
$\mathrm{Y} 1 \mathrm{C} 2$ & 0.55 \\
$\mathrm{Y} 2 \mathrm{C} 1$ & 0.58 \\
$\mathrm{Y} 2 \mathrm{C} 2$ & 0.57 \\
$\mathrm{Y} 3 \mathrm{C} 1$ & 0.75 \\
$\mathrm{Y} 3 \mathrm{C} 2$ & 0.68 \\
\hline
\end{tabular}

Table S10. The relative percentage of the eight subtypes in GU, GG, and GI, respectively.

\begin{tabular}{lllll}
\hline $\begin{array}{l}\text { Structural } \\
\text { Type }\end{array}$ & & GU $(\%)$ & GG $(\%)$ & GI $(\%)$ \\
\hline Type I-1 & A & 7.92 & 0 & 1.02 \\
Type I-2 & B & 17.82 & 4.55 & 20.40 \\
Type I-3 & C & 6.41 & 0 & 0 \\
Type II-1 & D & 3.74 & 0 & 8.49 \\
Type II-2 & E & 32.90 & 0 & 2.96 \\
Type II-3 & F & 17.04 & 0 & 1.48 \\
Type III-1 & G & 14.17 & 75.70 & 65.65 \\
Type III-2 & H & 0 & 19.75 & 0 \\
\hline
\end{tabular}



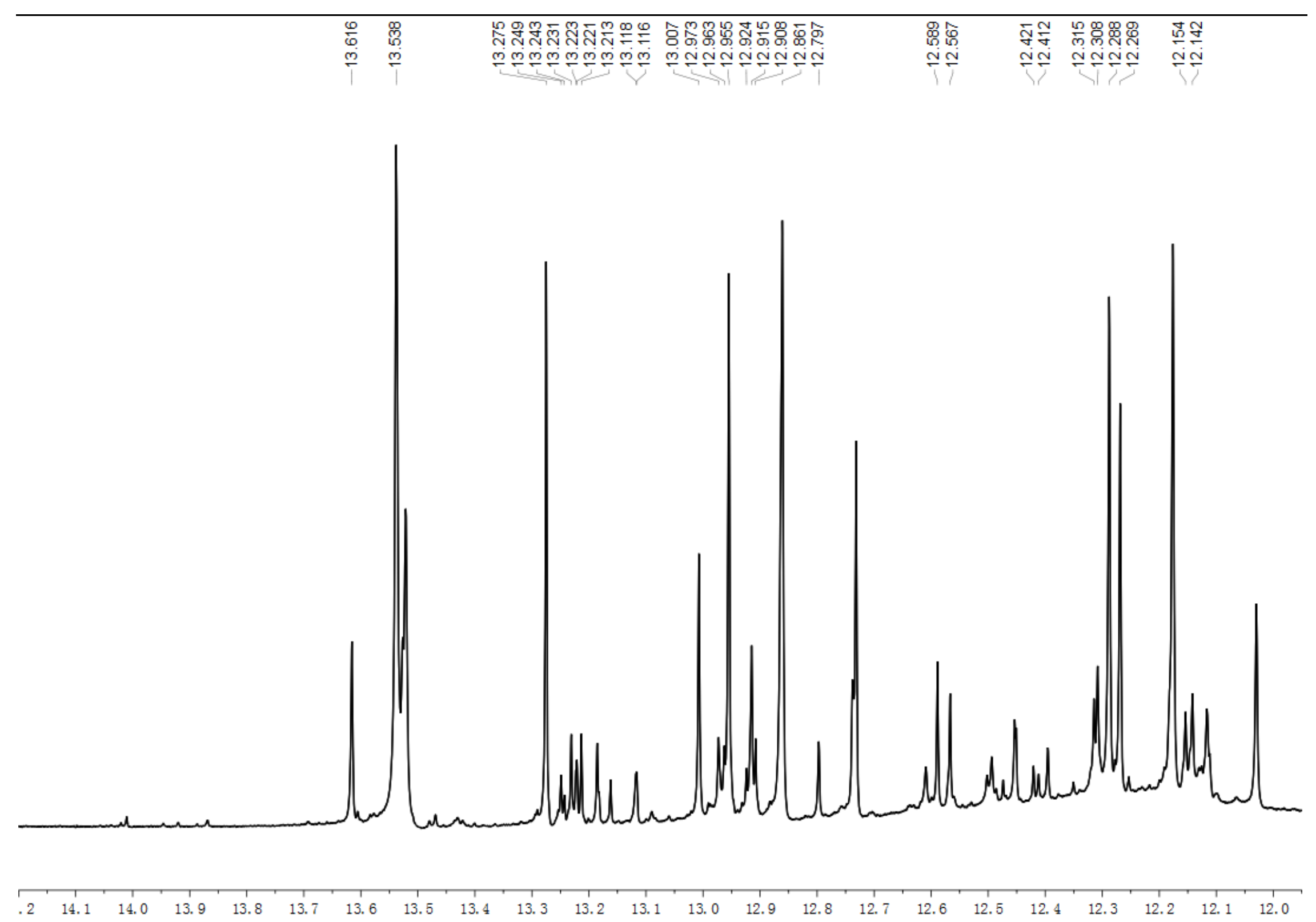

Figure S11. The ${ }^{1} \mathrm{H}$ NMR spectrum of GU-MF in DMSO- $d_{6}$. 


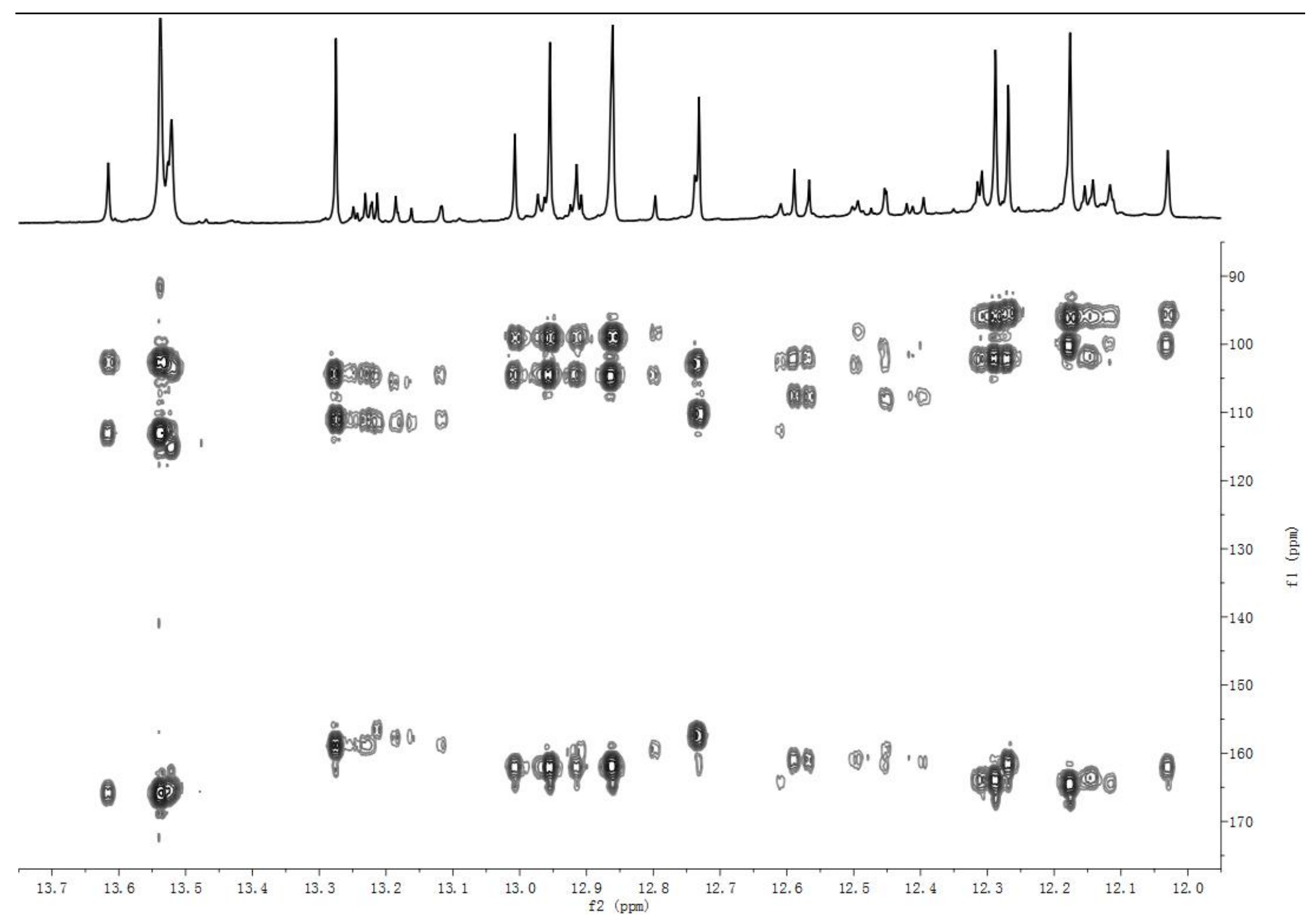

Figure S12. The HMBC spectrum of GU-MF in DMSO- $d_{6}$. 


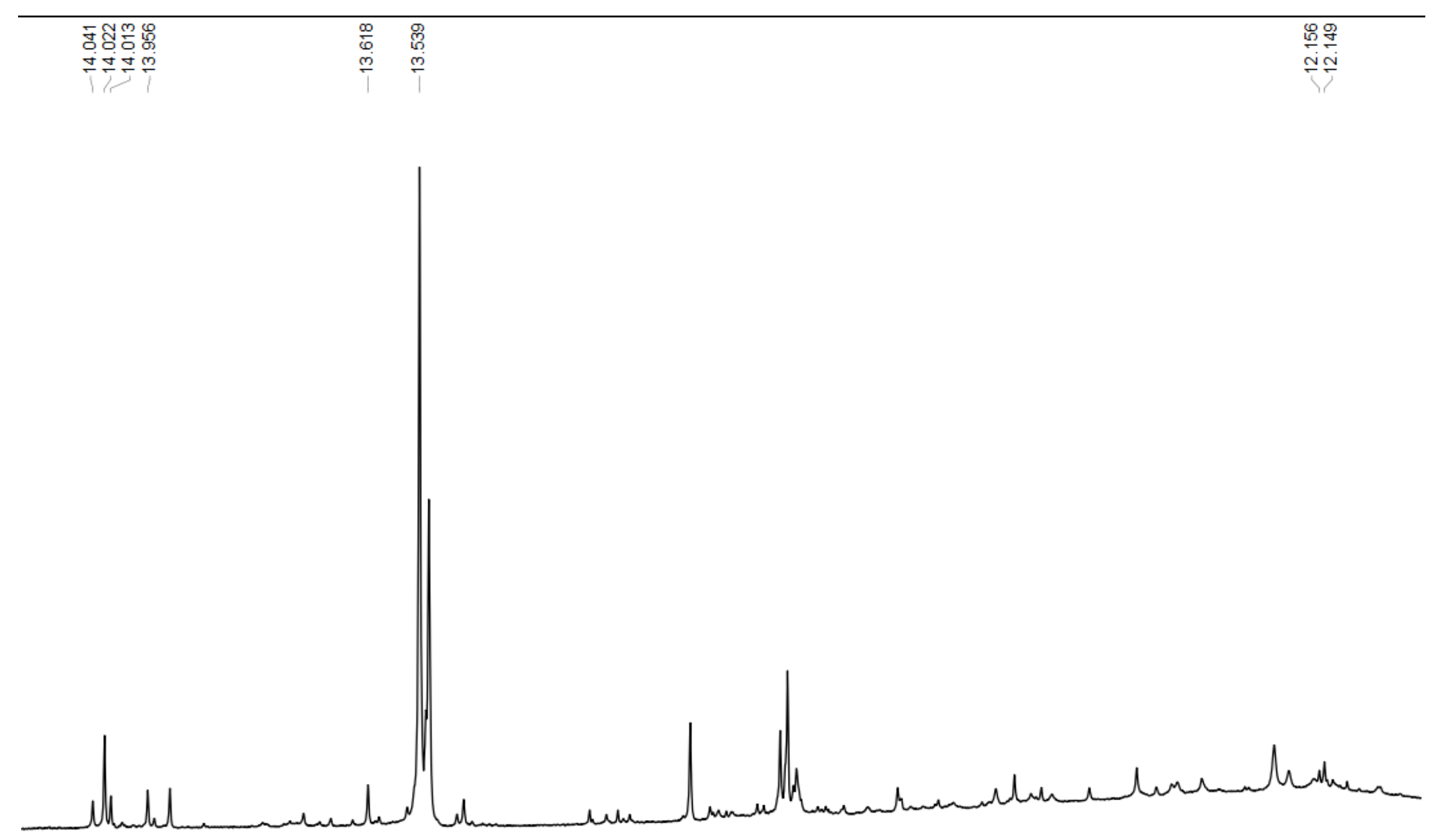

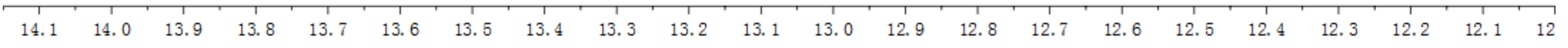

Figure S13. The ${ }^{1} \mathrm{H}$ NMR spectrum of GG-MF in DMSO- $d_{6}$. 


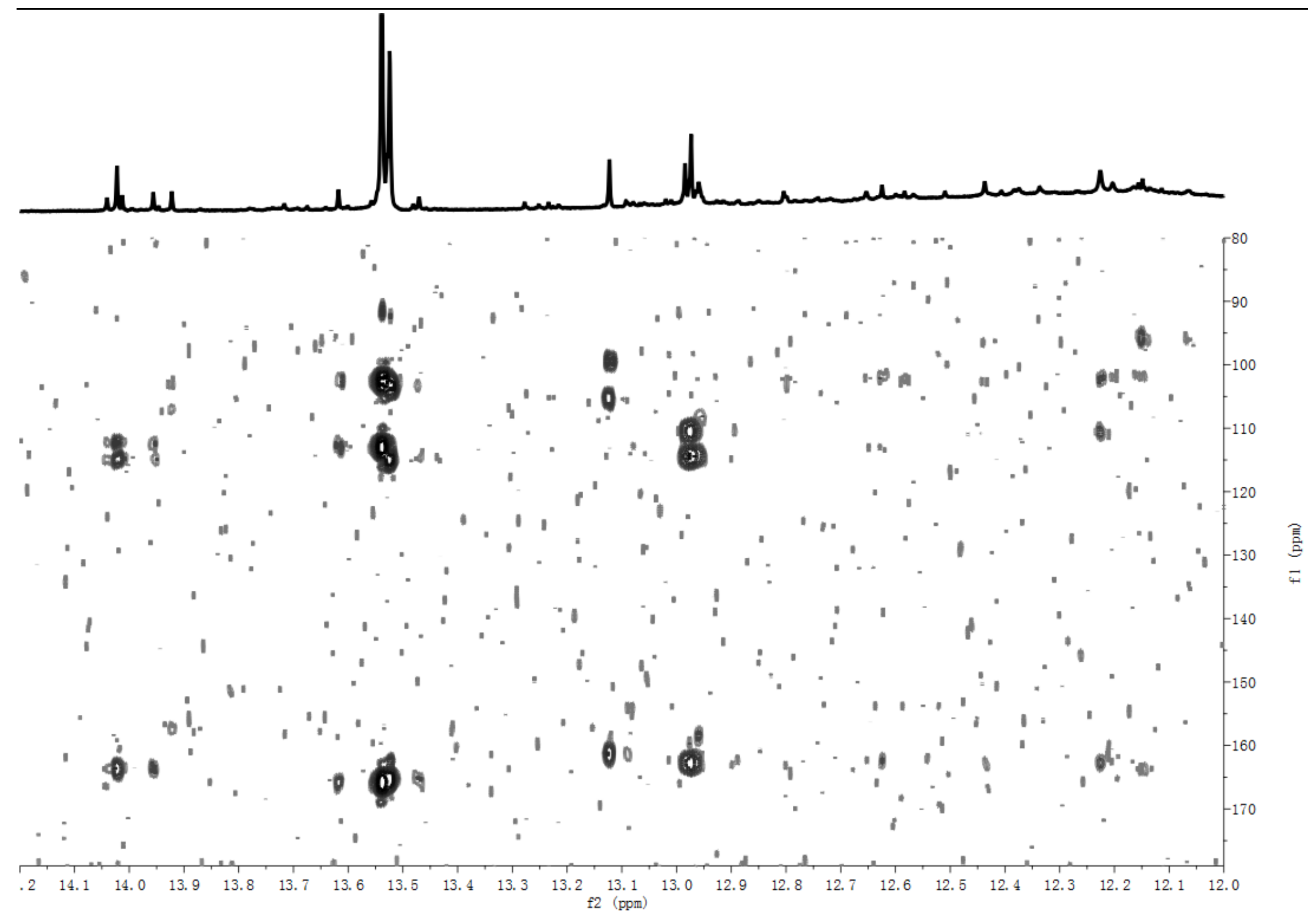

Figure S14. The HMBC spectrum of GG-MF in DMSO- $d_{6}$. 


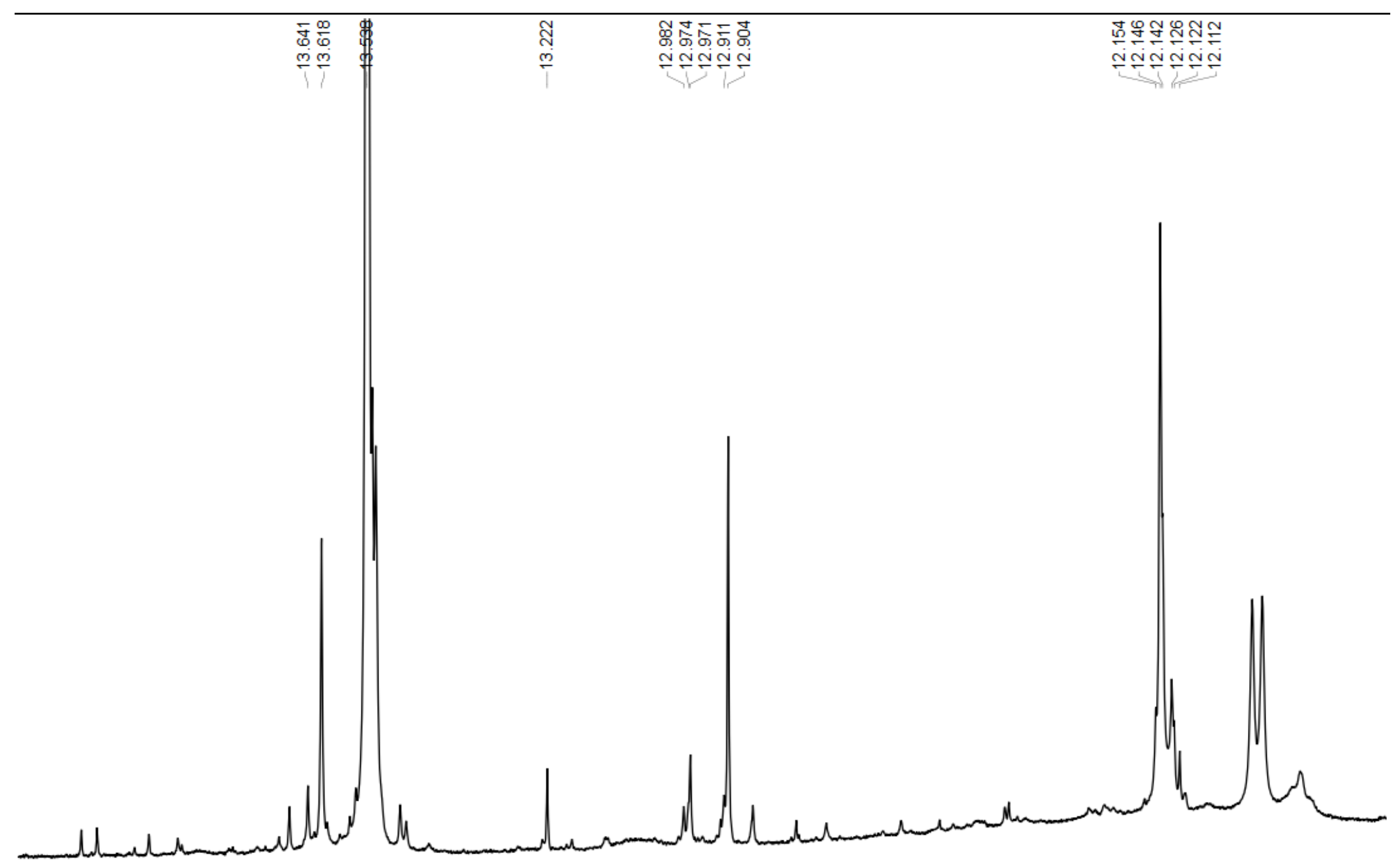

$\begin{array}{llllllllllllllllllllllll}14.1 & 14.0 & 13.9 & 13.8 & 13.7 & 13.6 & 13.5 & 13.4 & 13.3 & 13.2 & 13.1 & 13.0 & 12.9 & 12.8 & 12.7 & 12.6 & 12.5 & 12.4 & 12.3 & 12.2 & 12.1 & 12.0 & 11.9 & 11.8\end{array}$

Figure S15. The ${ }^{1} \mathrm{H}$ NMR spectrum of GI-MF in DMSO- $d_{6}$. 


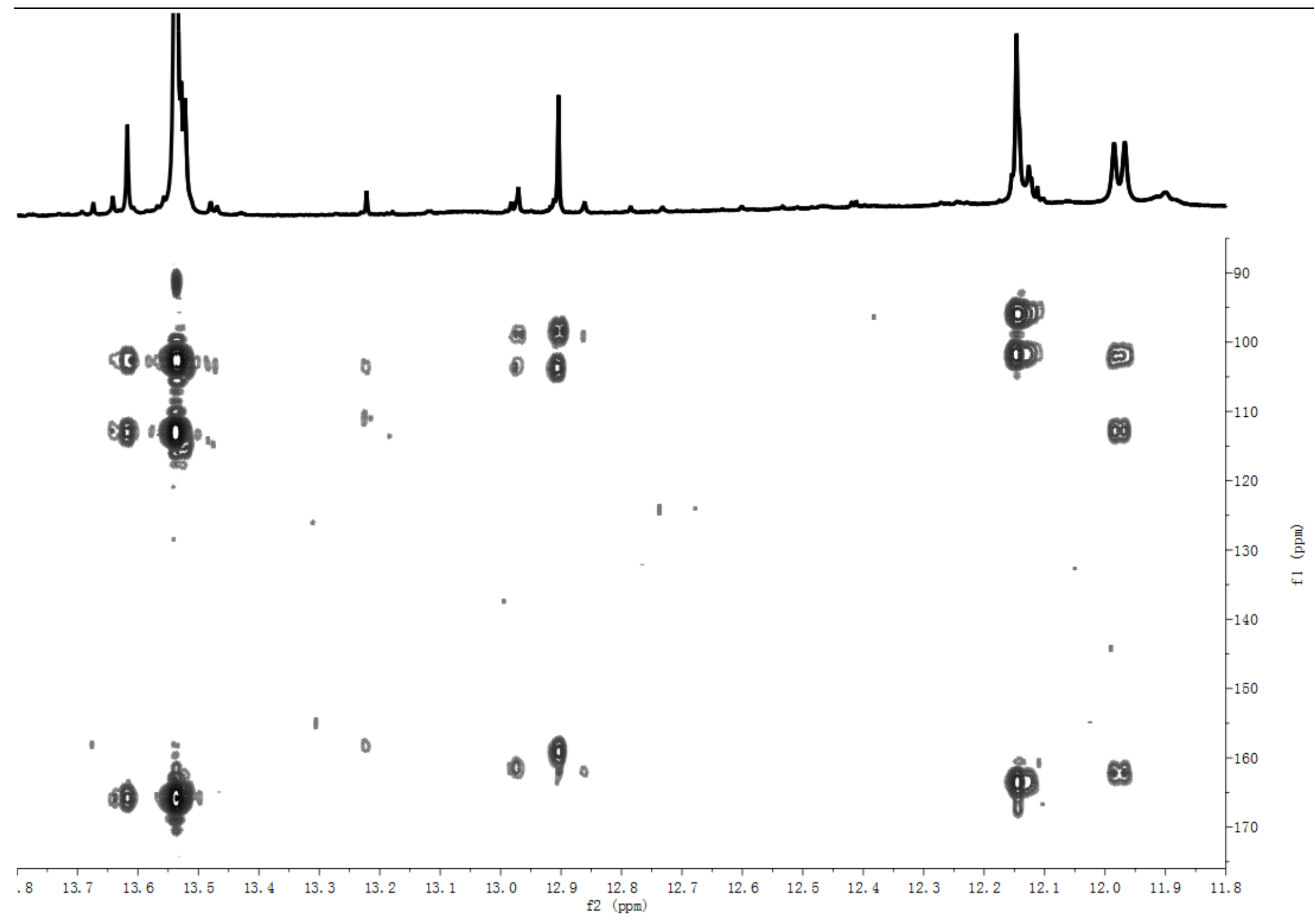

Figure S16. The HMBC spectrum of GI-MF in DMSO- $d_{6}$. 

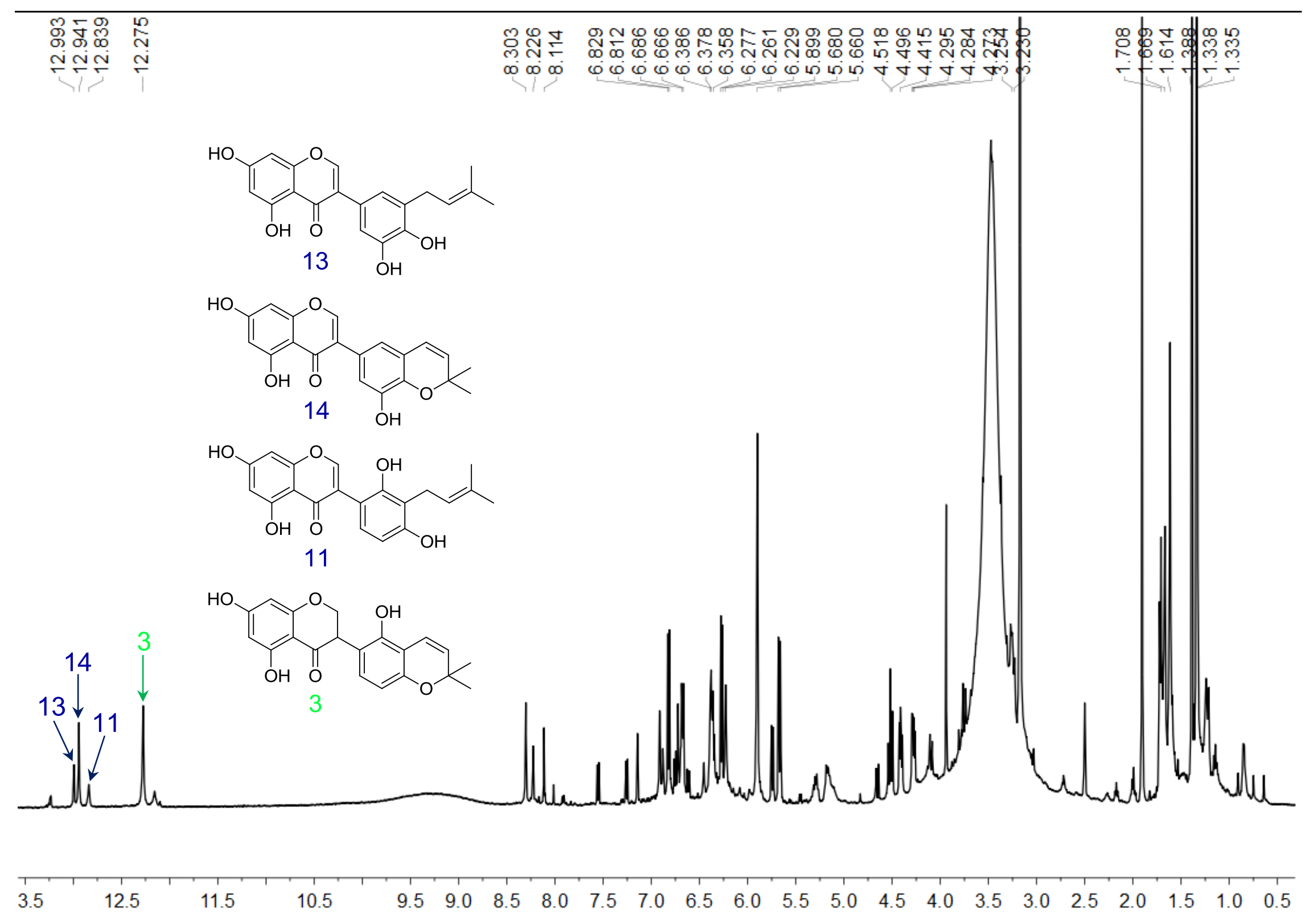

Figure S17. The ${ }^{1}$ H NMR spectrum of GU-MF-11 in DMSO- $d_{6}$. 


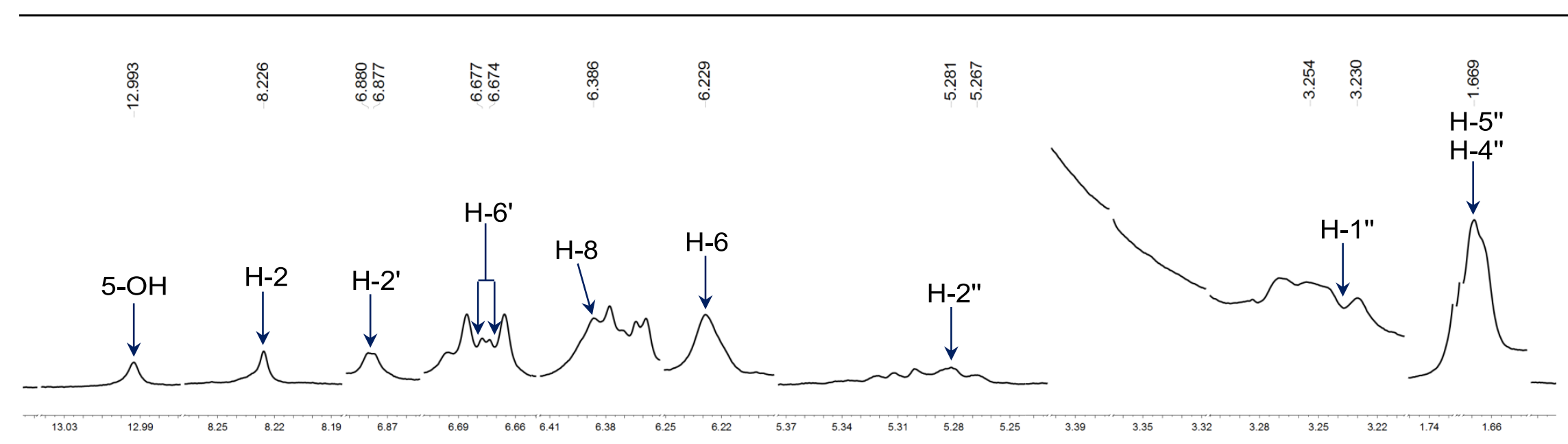

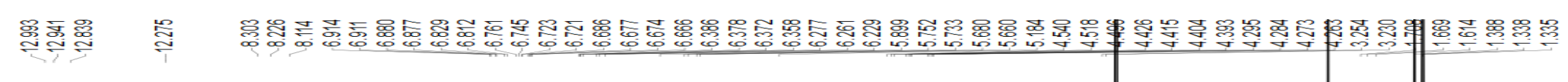
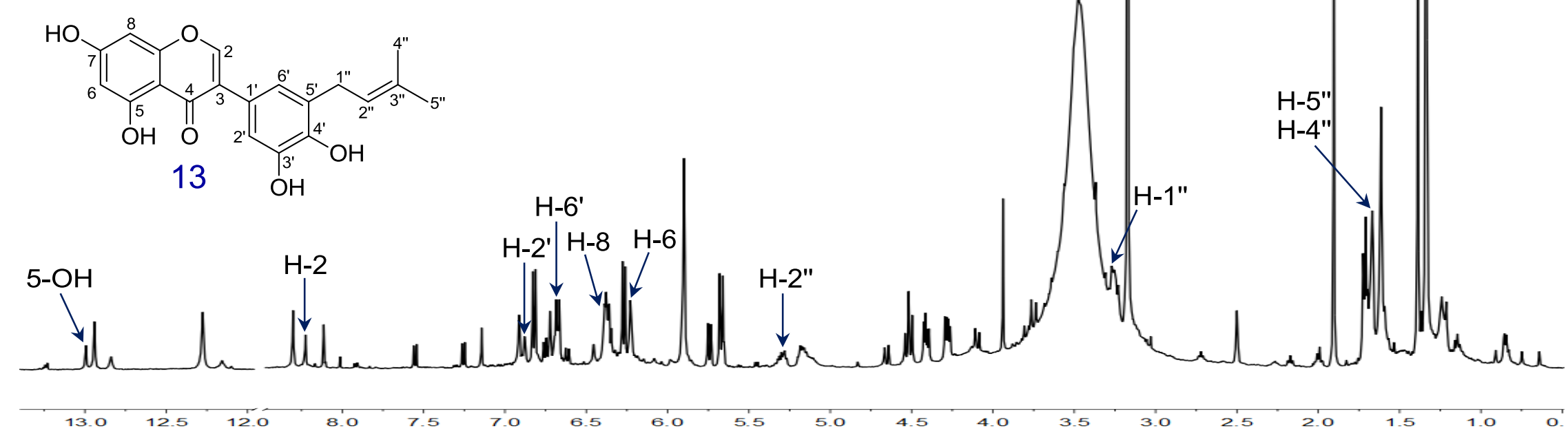

Figure S18. The ${ }^{1} \mathrm{H}$ NMR spectrum of compound 13 in GU-MF-11 in DMSO- $d_{6}$. 


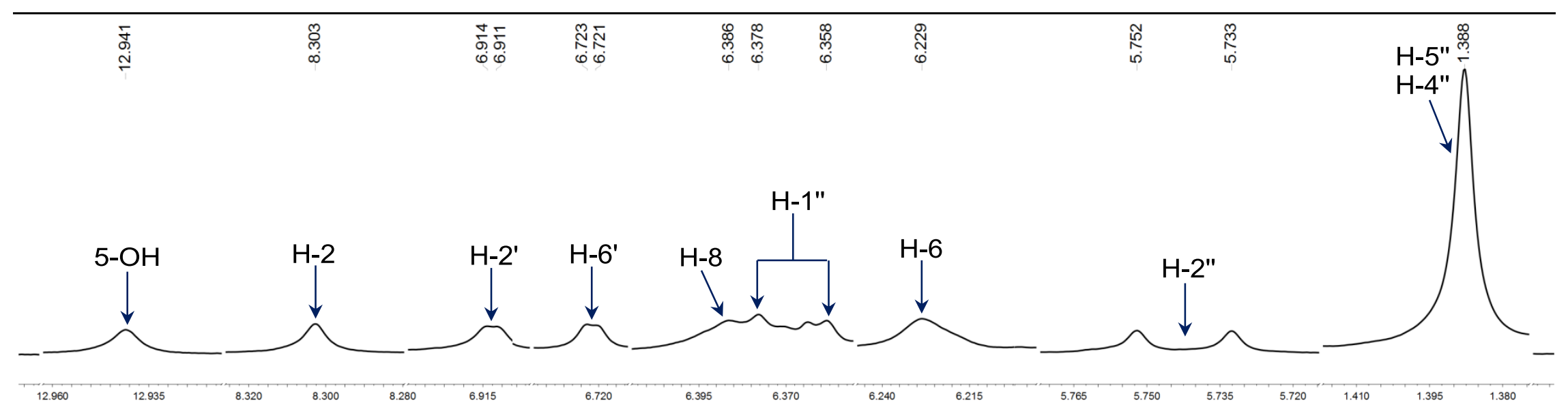

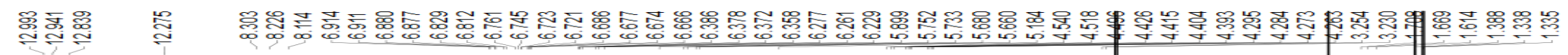
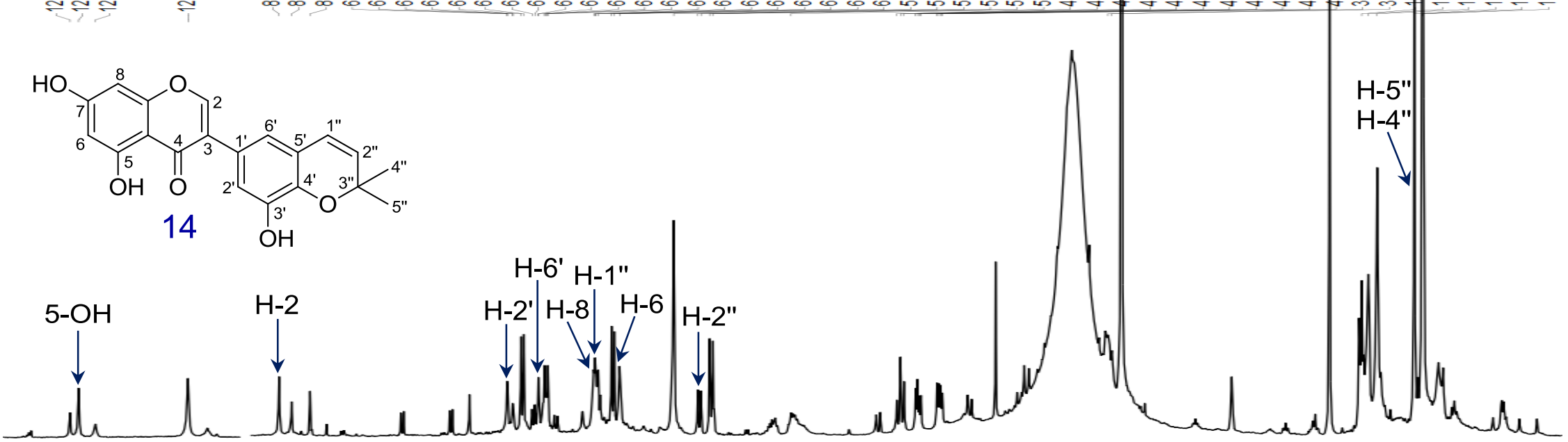

Figure S19. The ${ }^{1} \mathrm{H}$ NMR spectrum of compound $\mathbf{1 4}$ in GU-MF-11 in DMSO- $d_{6}$. 


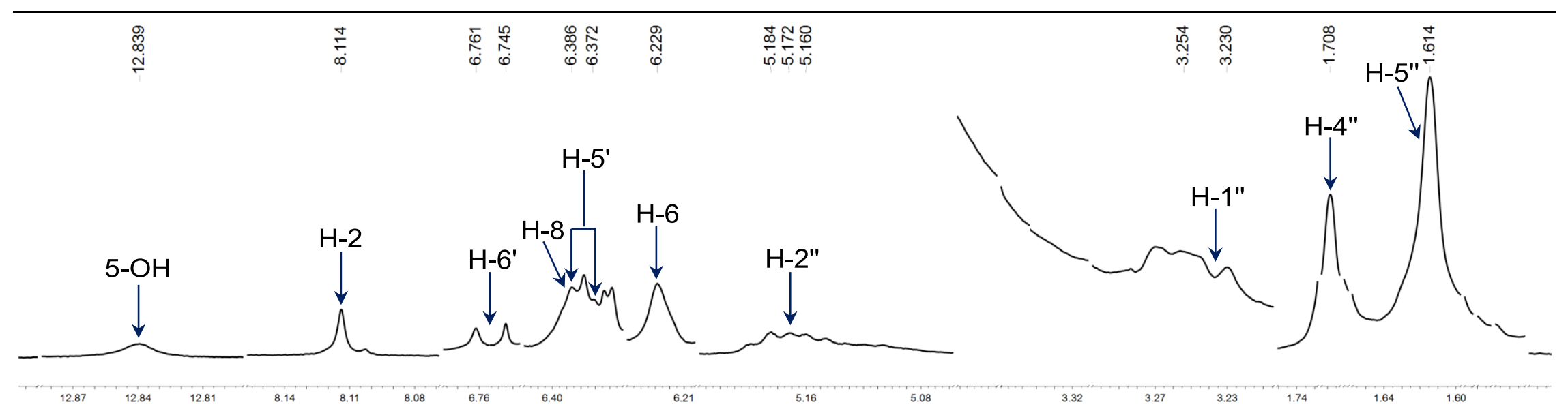

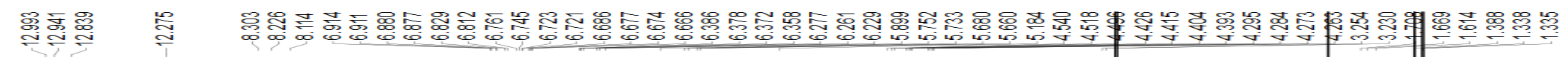

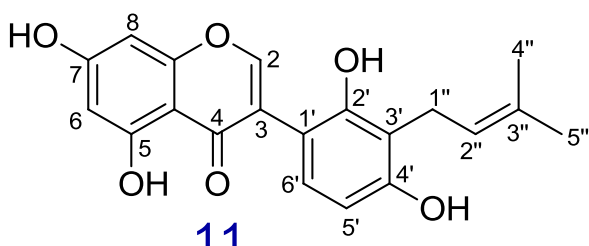

11
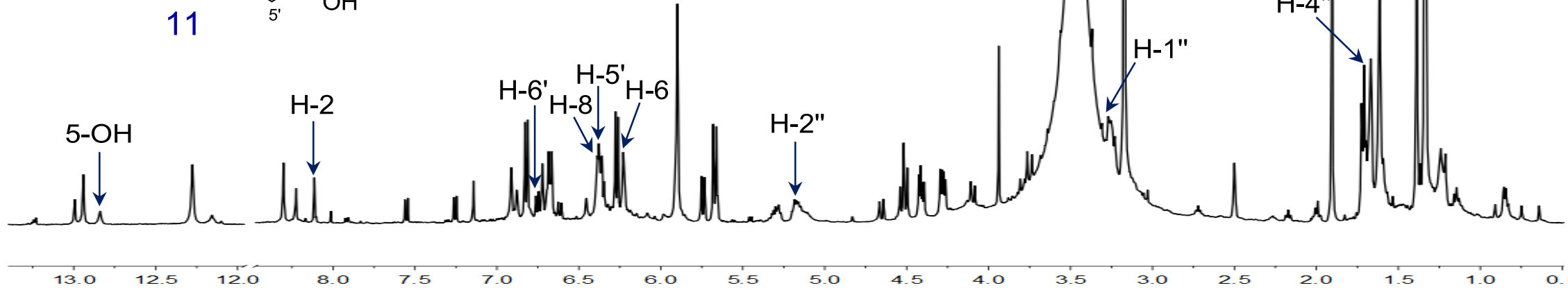

Figure S20. The ${ }^{1} \mathrm{H}$ NMR spectrum of compound $\mathbf{1 1}$ in GU-MF-11 in DMSO- $d_{6}$. 


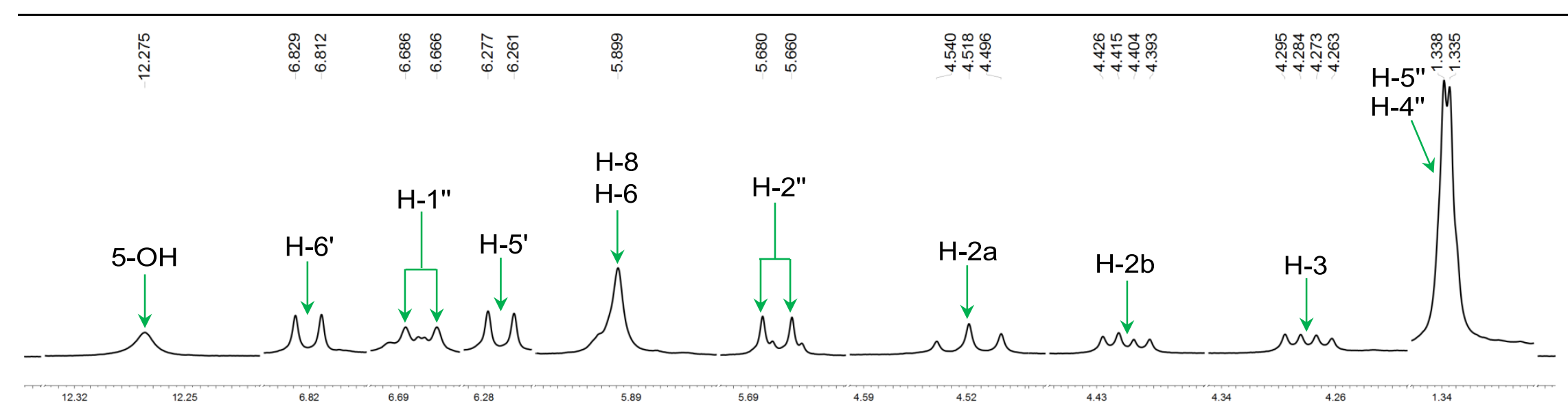

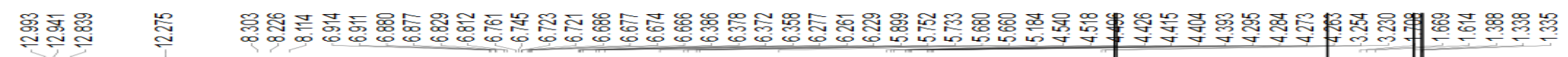
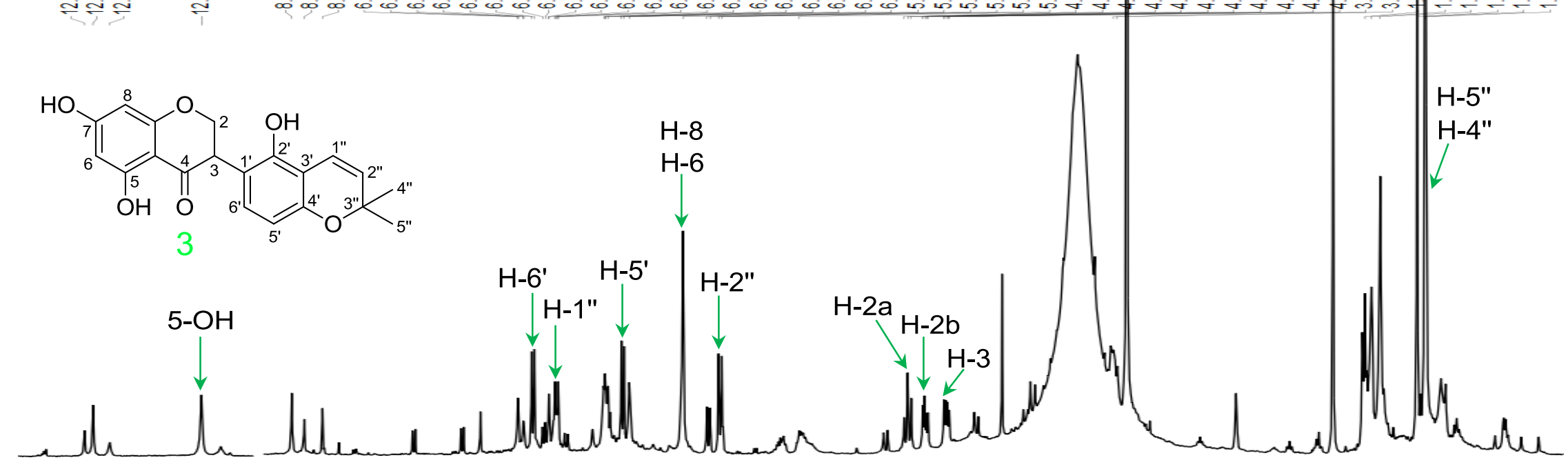

$13.0 \cdot 12.5120$

Figure S21. The ${ }^{1} \mathrm{H}$ NMR spectrum of compound 3 in GU-MF-11 in DMSO- $d_{6}$. 


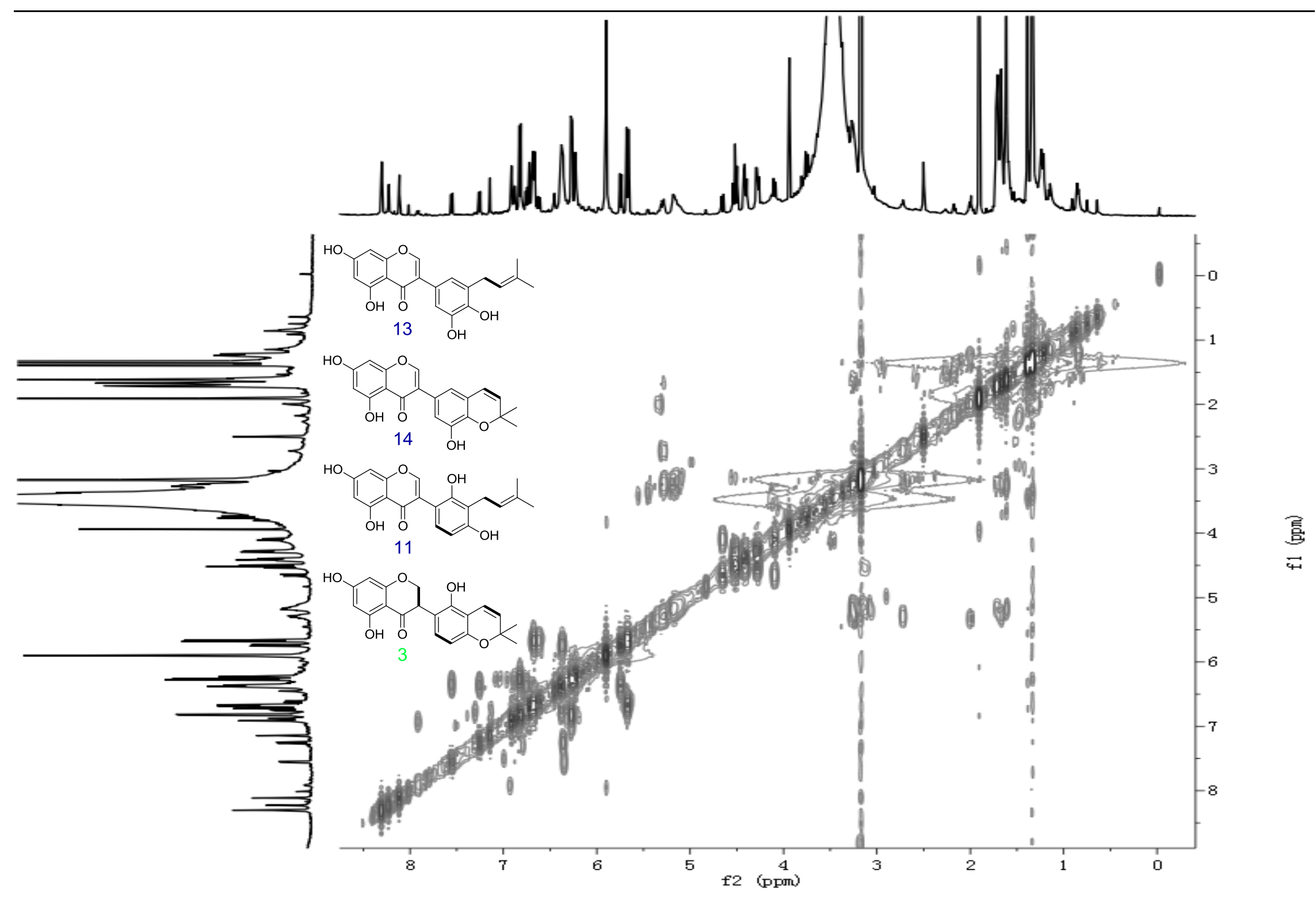

Figure S22. The ${ }^{1} \mathrm{H}-{ }^{1} \mathrm{H}$ COSY spectrum of GU-MF-11 in DMSO- $d_{6}$. 


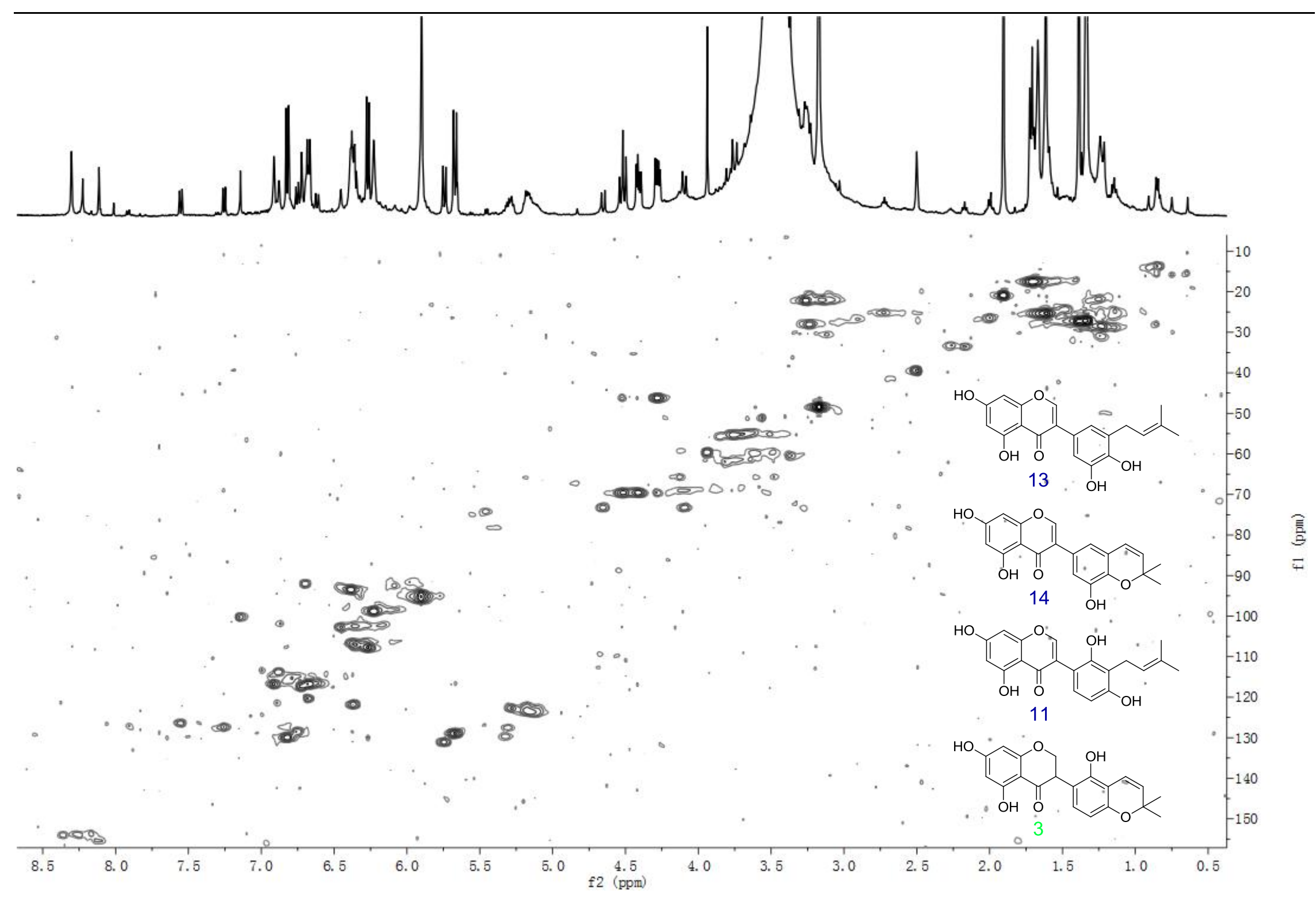

Figure S23. The HSQC spectrum of GU-MF-11 in DMSO- $d_{6}$. 


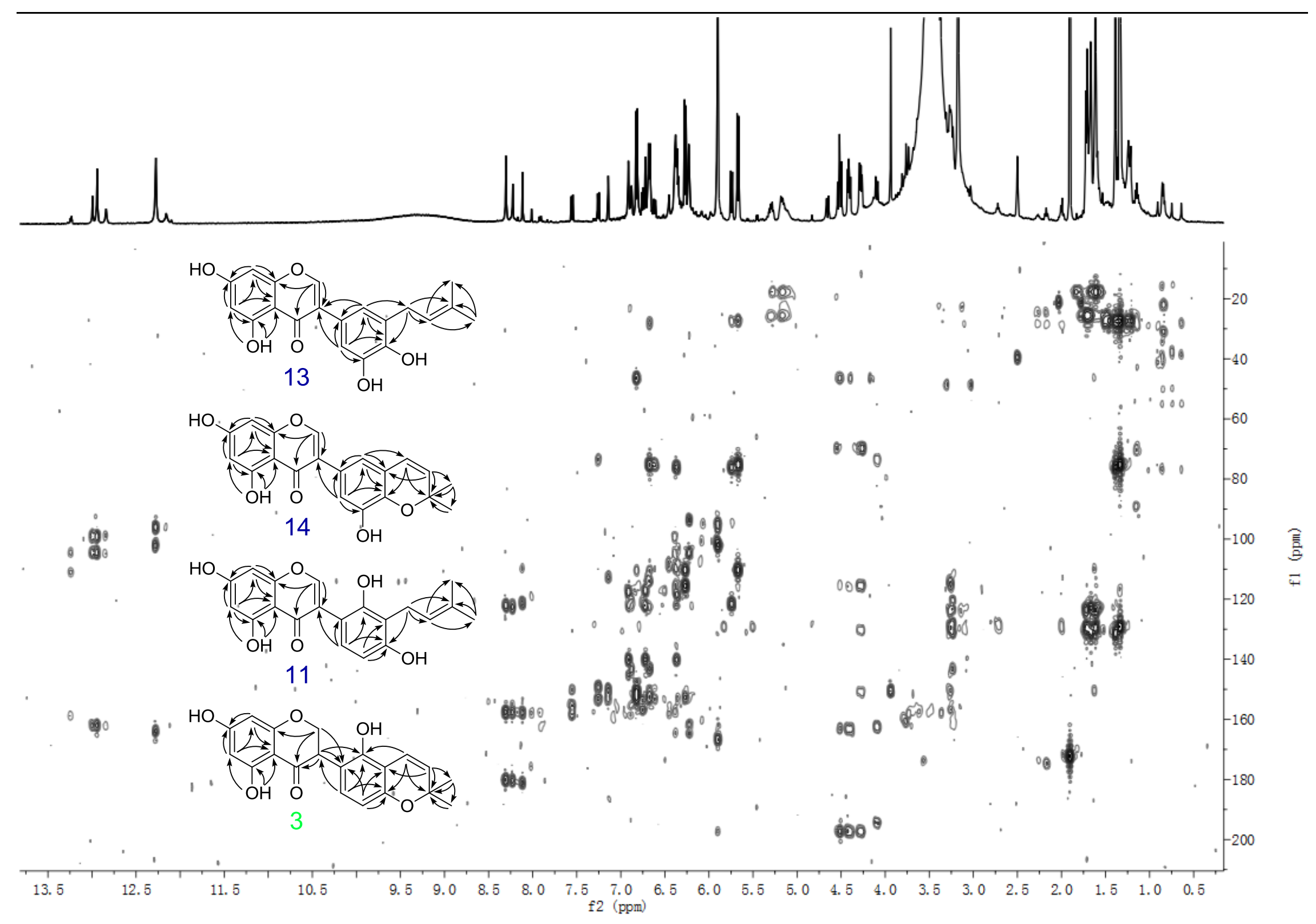

Figure S24. The HMBC spectrum of GU-MF-11 in DMSO- $d_{6}$. 

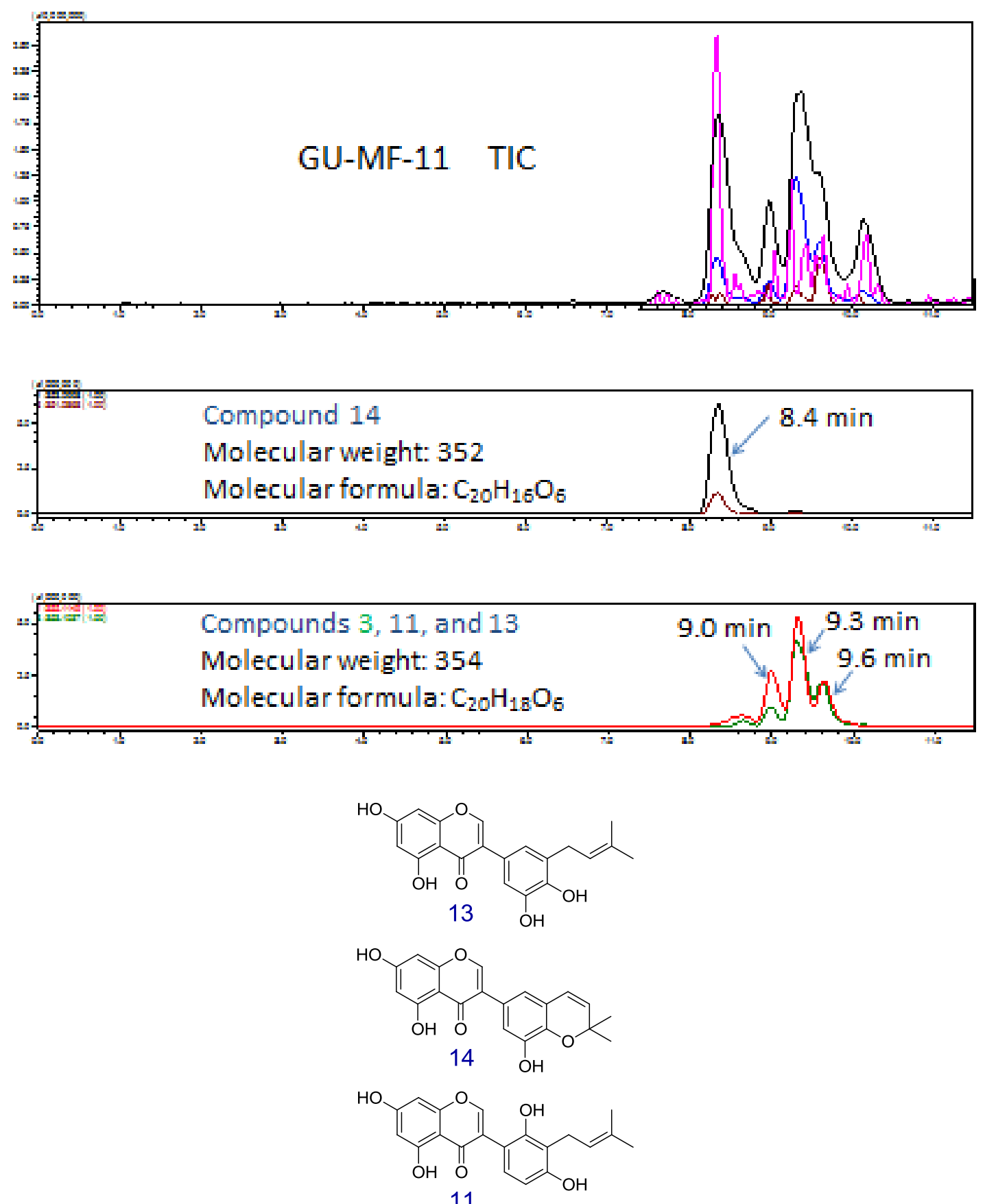

11

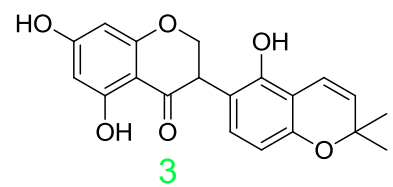

Figure S25. The IT-TOF TIC and mass chromatogram of GU-MF-11. 

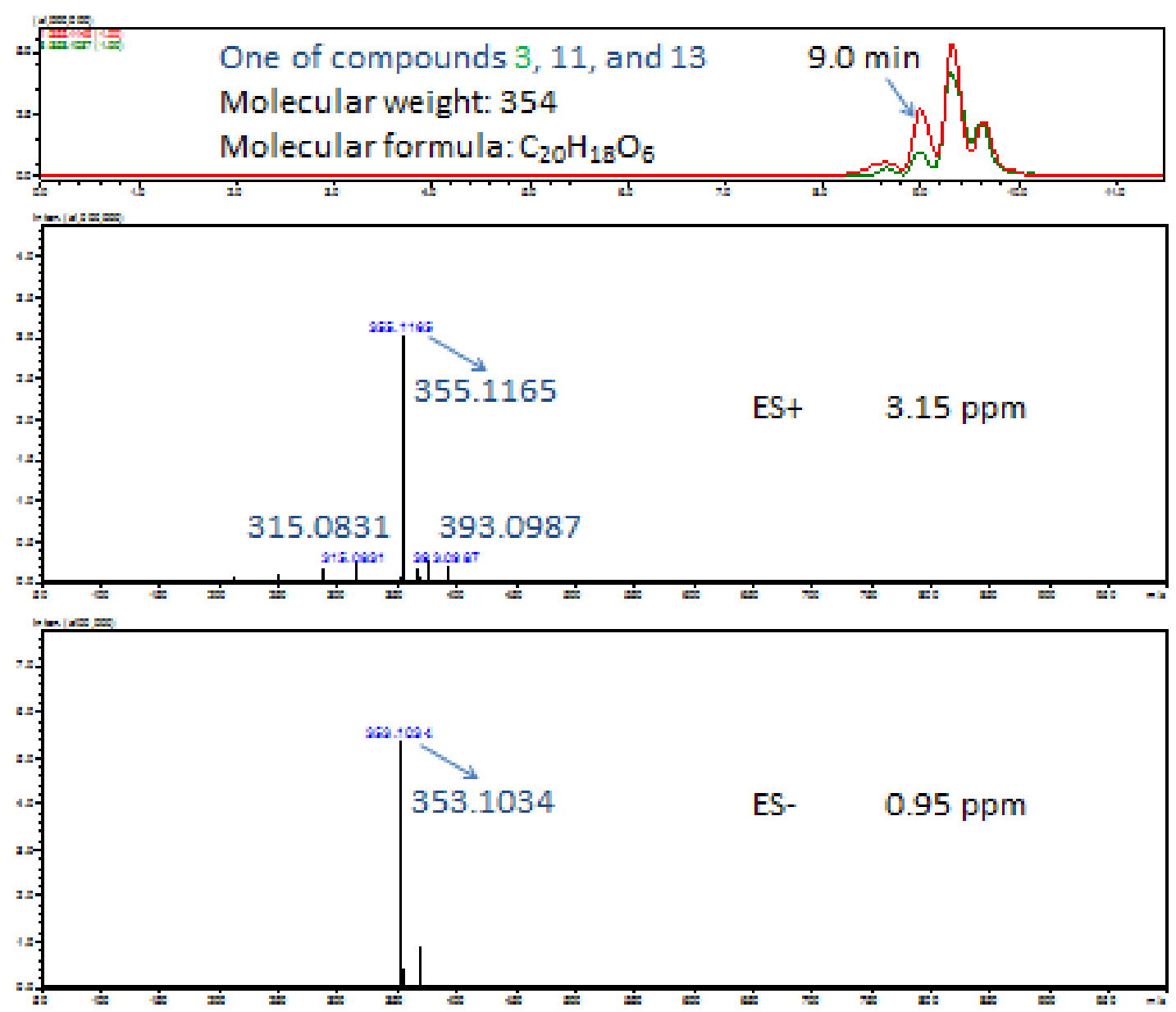<smiles>CC(C)=CCc1cc(-c2coc3cc(O)cc(O)c3c2=O)cc(O)c1O</smiles><smiles>CC(C)=CCc1c(O)ccc(-c2coc3cc(O)cc(O)c3c2=O)c1O</smiles><smiles>CC1(C)C=Cc2c(ccc(C3COc4cc(O)cc(O)c4C3=O)c2O)O1</smiles>

Figure S26. The (+)-HRESIMS and (-)-HRESIMS spectra of one of compounds 3, 11, and 13 in GU-MF-11 with extracted ions (positive and negative) for $m / z, 355$ and 353, respectively. 

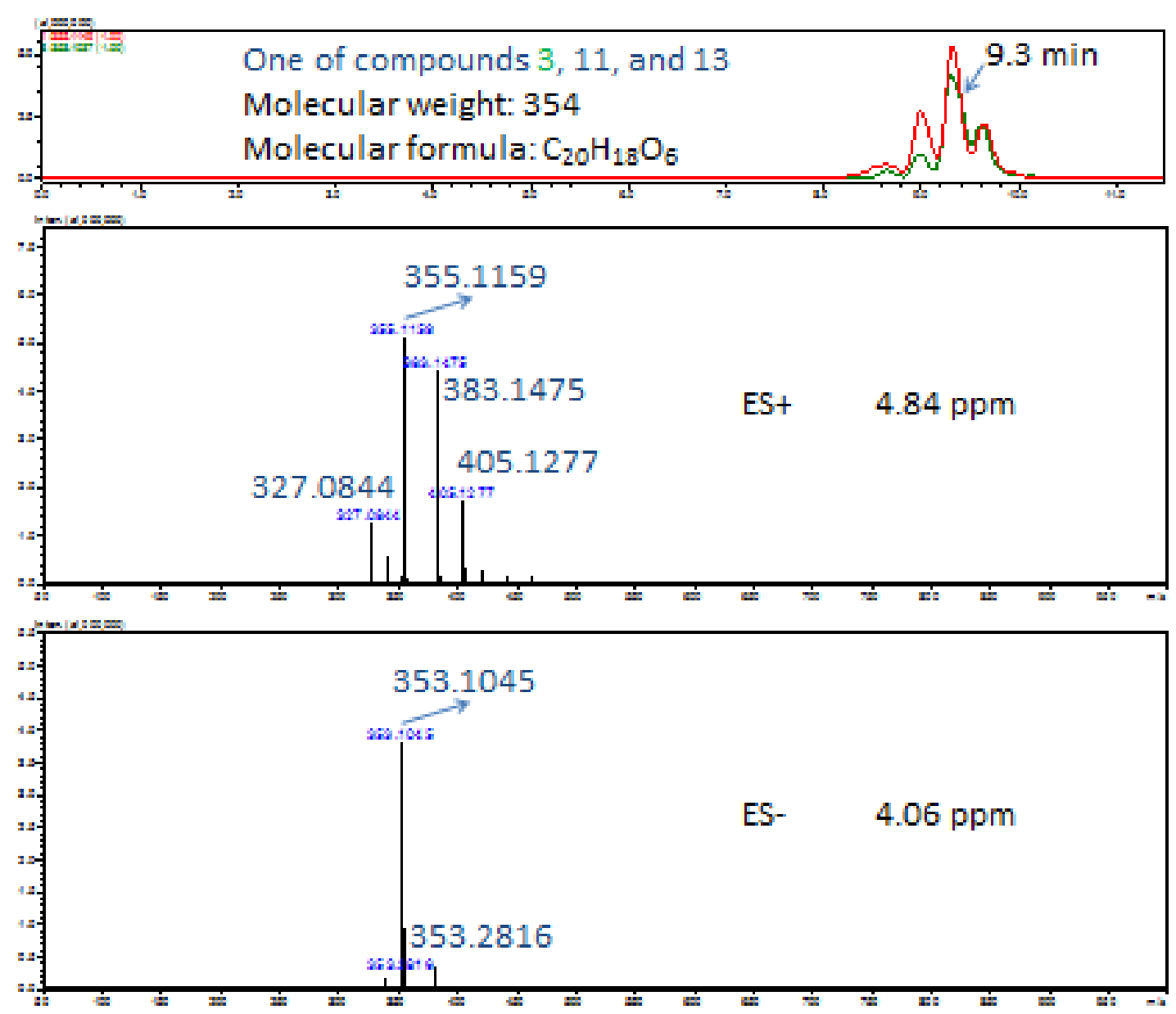<smiles>CC(C)=CCc1cc(-c2coc3cc(O)cc(O)c3c2=O)cc(O)c1O</smiles><smiles>CC(C)=CCc1c(O)ccc(-c2coc3cc(O)cc(O)c3c2=O)c1O</smiles><smiles>CC1(C)C=Cc2c(ccc(C3COc4cc(O)cc(O)c4C3=O)c2O)O1</smiles>

Figure S27. The (+)-HRESIMS and (-)-HRESIMS spectra of one of compounds 3, 11, and $\mathbf{1 3}$ in GU-MF-11 with extracted ions (positive and negative) for $m / z 355$ and 353, respectively. 

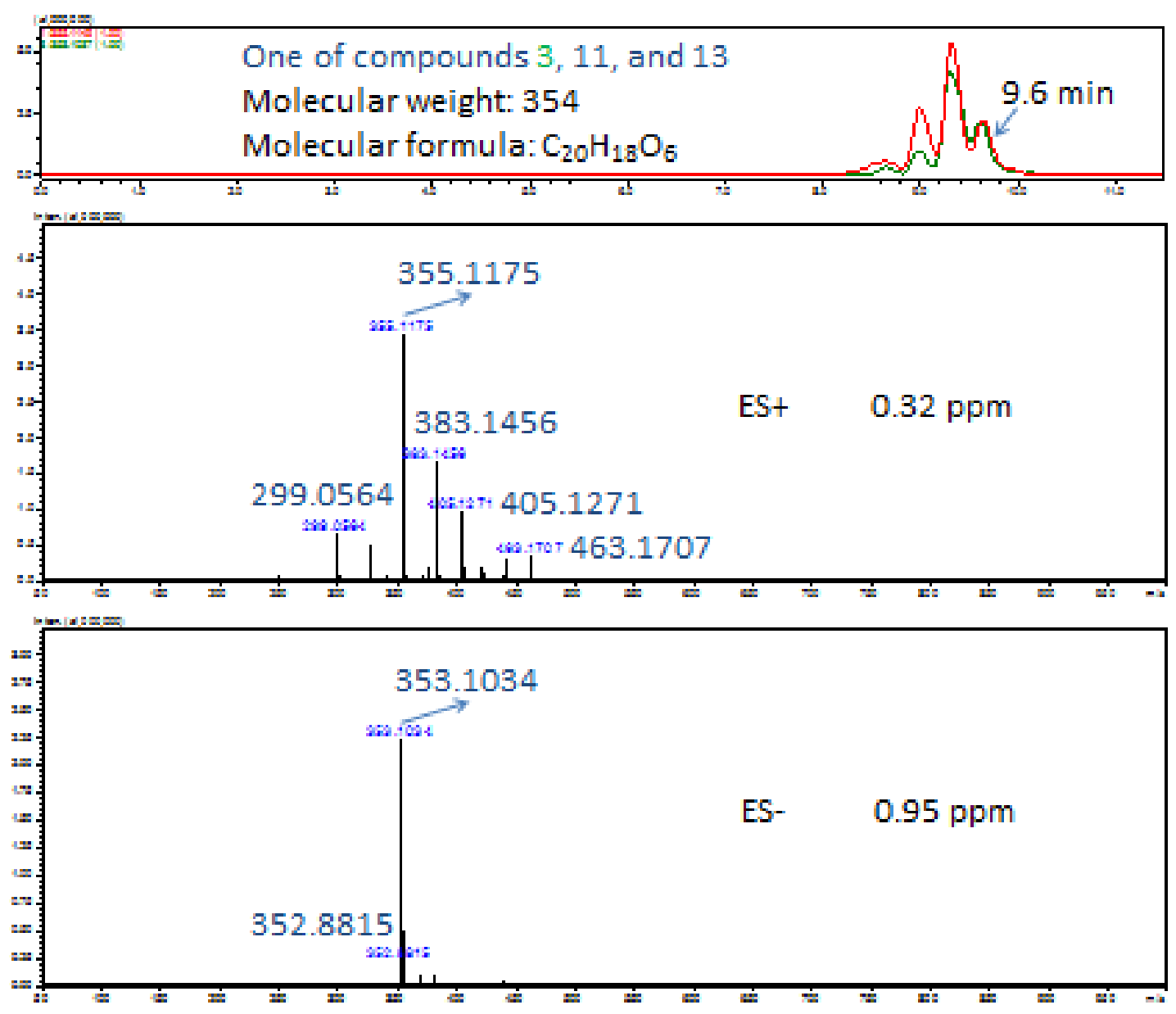<smiles>CC(C)=CCc1cc(-c2coc3cc(O)cc(O)c3c2=O)cc(O)c1O</smiles><smiles>CC(C)=CCc1c(O)ccc(-c2coc3cc(O)cc(O)c3c2=O)c1O</smiles><smiles>CC1(C)C=Cc2c(ccc(C3COc4cc(O)cc(O)c4C3=O)c2O)O1</smiles>

Figure S28. The (+)-HRESIMS and (-)-HRESIMS spectra of one of compounds 3, 11, and 13 in GU-MF-11 with extracted ions (positive and negative) for $m / z, 355$ and 353, respectively. 

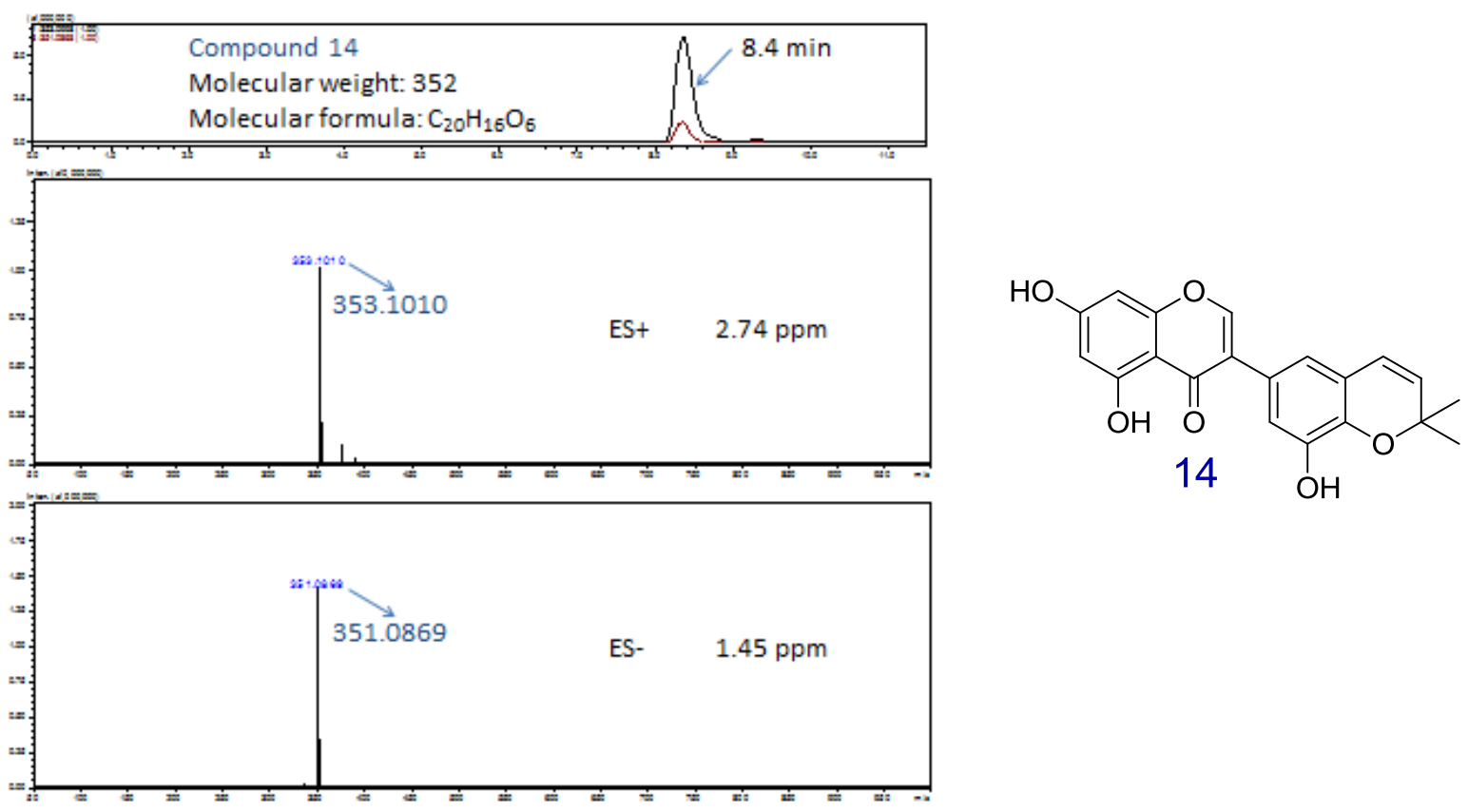

Figure S29. The (+)-HRESIMS and (-)-HRESIMS spectra of compound 14 in GU-MF-11 with extracted ions (positive and negative) for $m / z 353$ and 351, respectively. 
సั

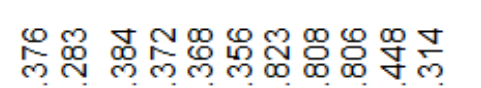

$\infty \infty$ NMN

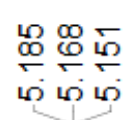

ल⿸户㔾

串

$\stackrel{2}{\leftarrow}$
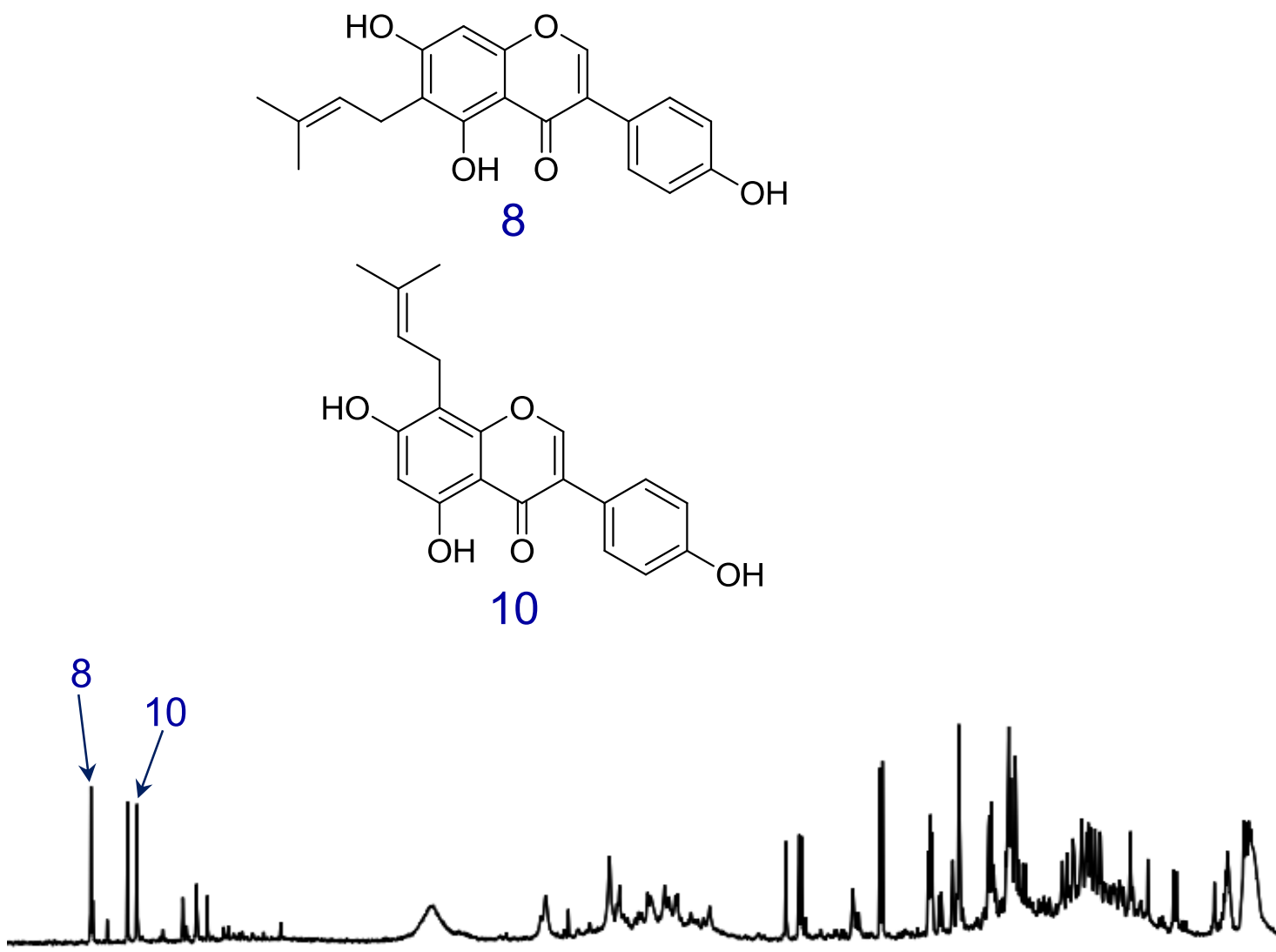

13.0

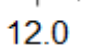

11.0

10.0

9.0

8.0

7.0

6.0

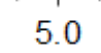

4.0

3.0

2.0

1.0

Figure S30. The ${ }^{1} \mathrm{H}$ NMR spectrum of GU-MF-12 in DMSO- $d_{6}$. 


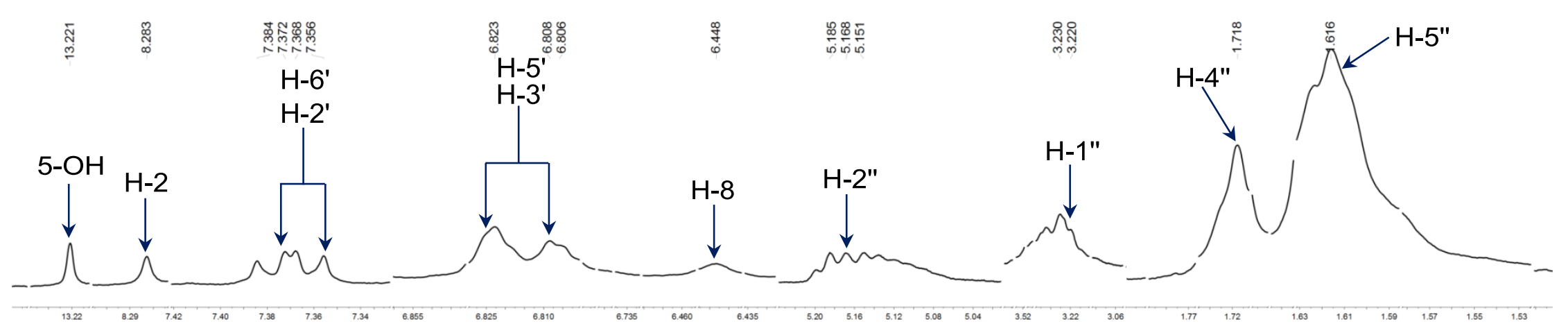

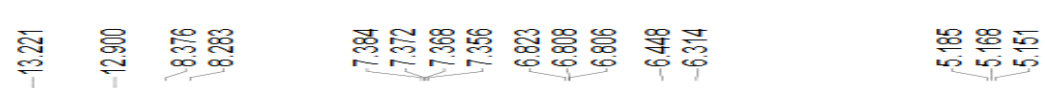

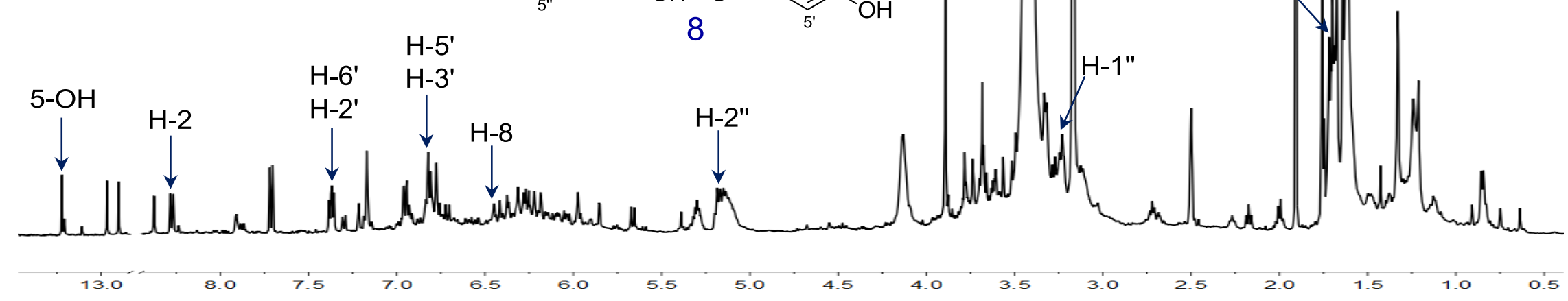

Figure S31. The ${ }^{1} \mathrm{H}$ NMR spectrum of compound 8 in GU-MF-12 in DMSO- $d_{6}$. 

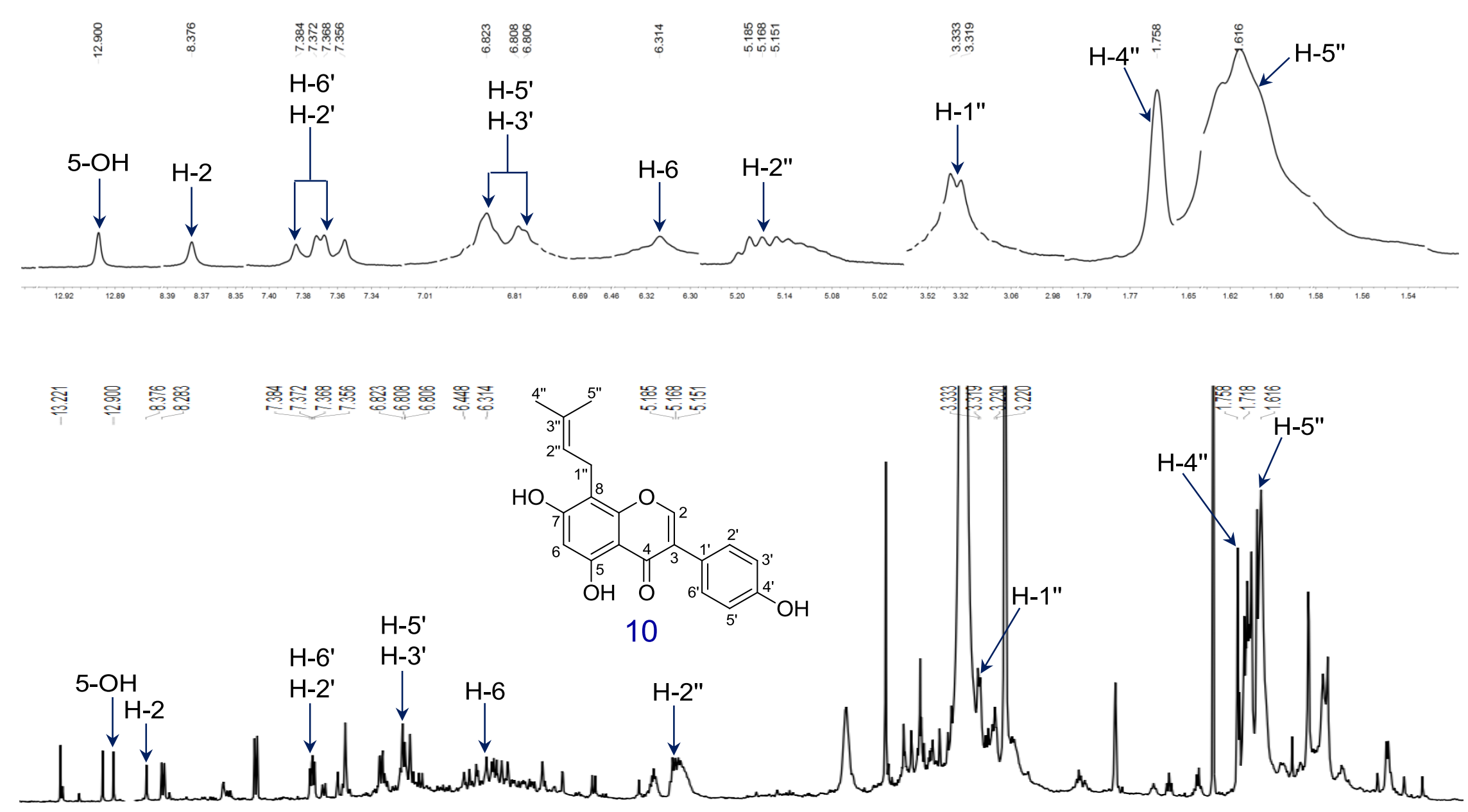

Figure S32. The ${ }^{1} \mathrm{H}$ NMR spectrum of compound 10 in GU-MF-12 in DMSO- $d_{6}$. 


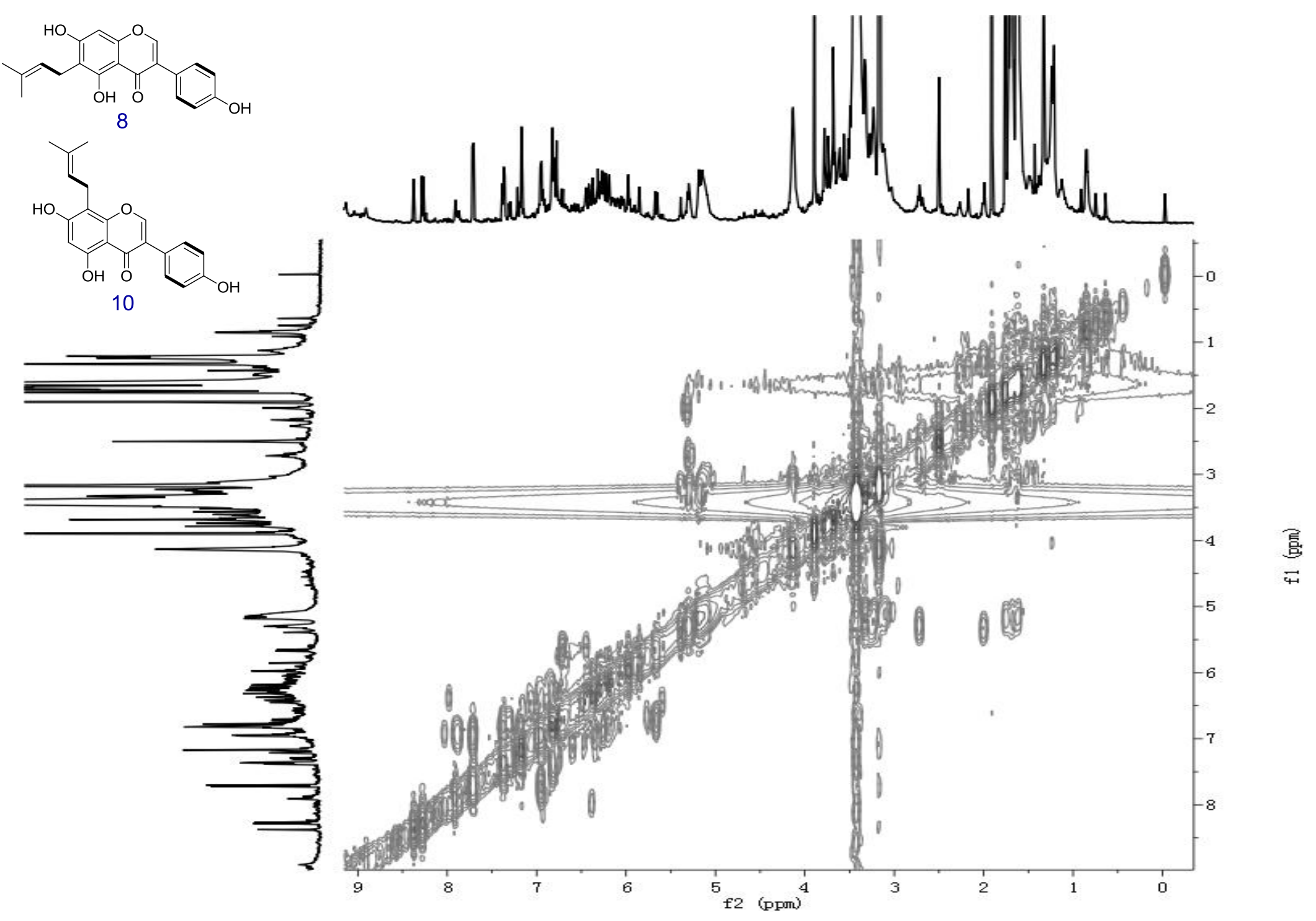

Figure S33. The ${ }^{1} \mathrm{H}-{ }^{1} \mathrm{H}$ COSY spectrum of GU-MF-12 in DMSO- $d_{6}$. 


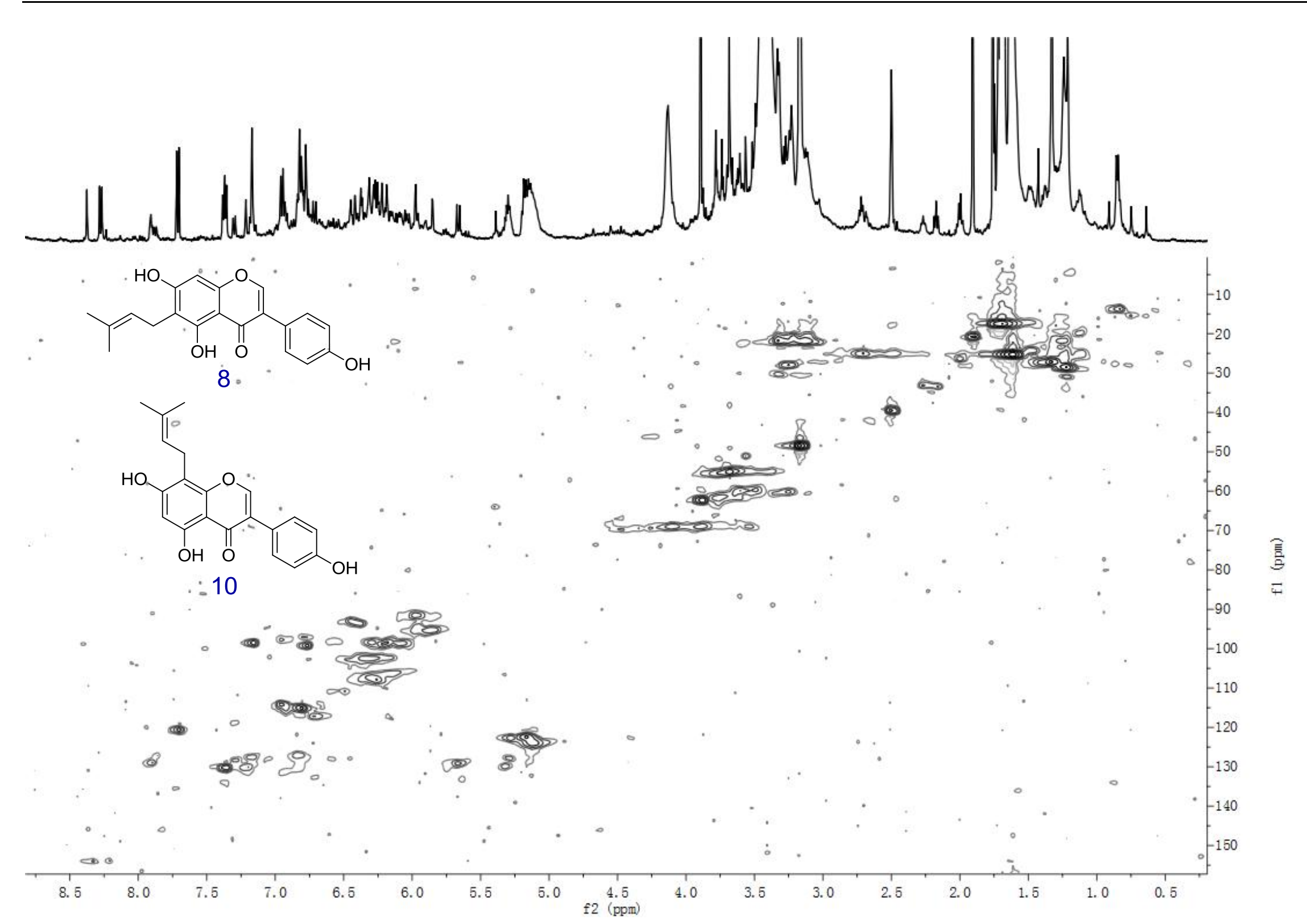

Figure S34. The HSQC spectrum of GU-MF-12 in DMSO- $d_{6}$.

60 


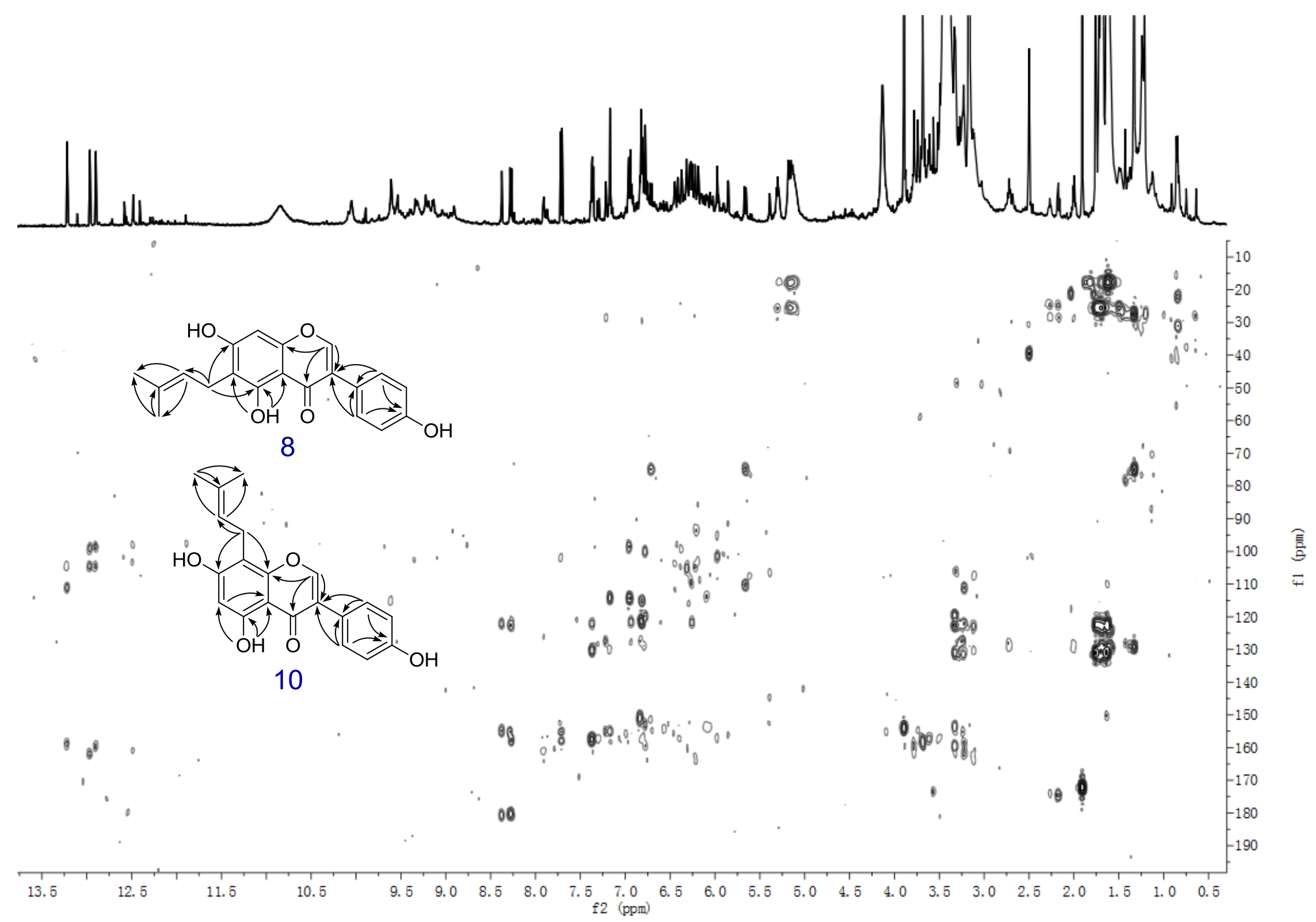

Figure S35. The HMBC spectrum of GU-MF-12 in DMSO- $d_{6}$. 

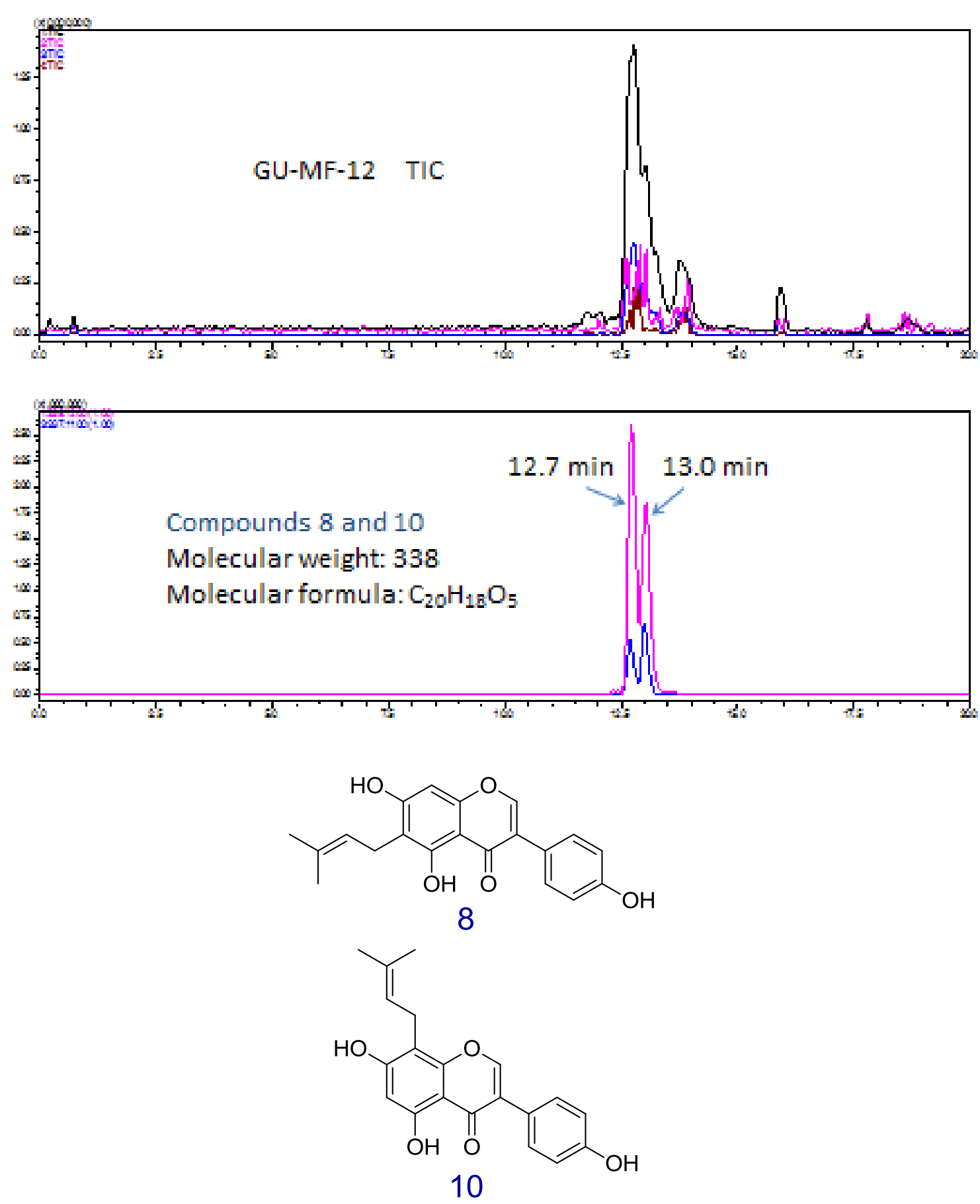

Figure S36. The IT-TOF TIC and mass chromatogram of GU-MF-12. 

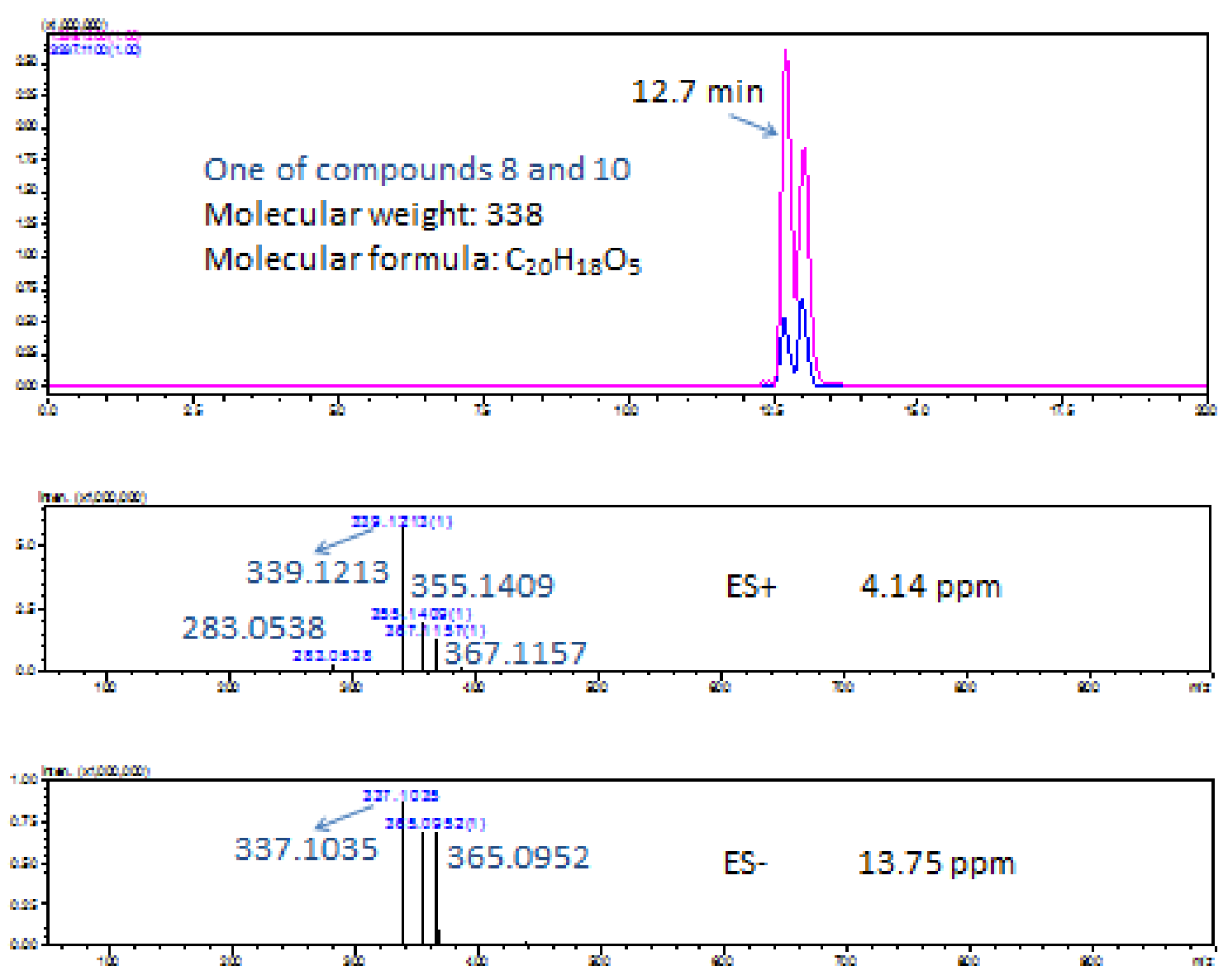<smiles>CC(C)=CCc1c(O)cc2occ(-c3ccc(O)cc3)c(=O)c2c1O</smiles><smiles>CC(C)=CCc1c(O)cc(O)c2c(=O)c(-c3ccc(O)cc3)coc12</smiles>

Figure S37. The (+)-HRESIMS and (-)-HRESIMS spectra of one of compounds 8 and $\mathbf{1 0}$ in GU-MF-12 with extracted ions (positive and negative) for $m / z, 339$ and 337, respectively. 

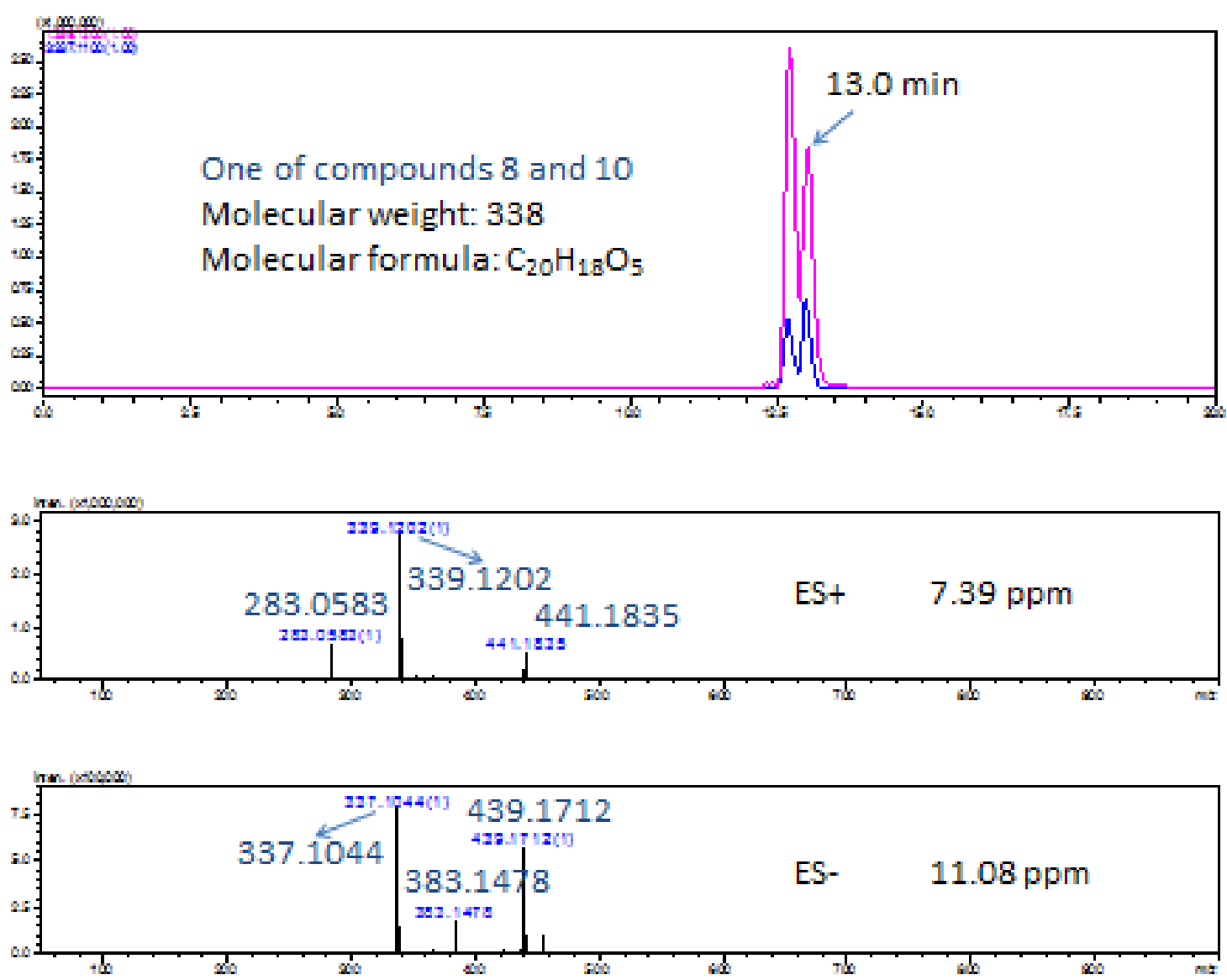<smiles>CC(C)=CCc1c(O)cc2occ(-c3ccc(O)cc3)c(=O)c2c1O</smiles><smiles>CC(C)=CCc1c(O)cc(O)c2c(=O)c(-c3ccc(O)cc3)coc12</smiles>

Figure S38. The (+)-HRESIMS and (-)-HRESIMS spectra of one of the compounds 8 and $\mathbf{1 0}$ in GU-MF-12 with extracted ions (positive and negative) for $m / z 339$ and 337, respectively. 


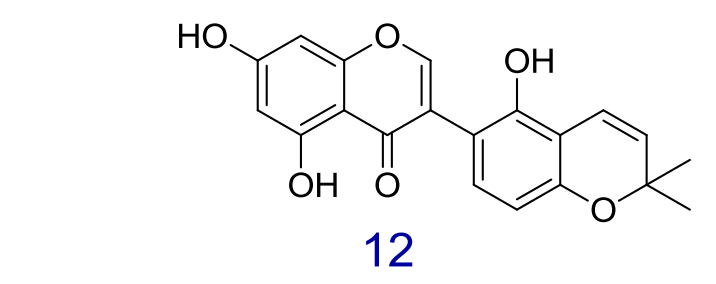

12
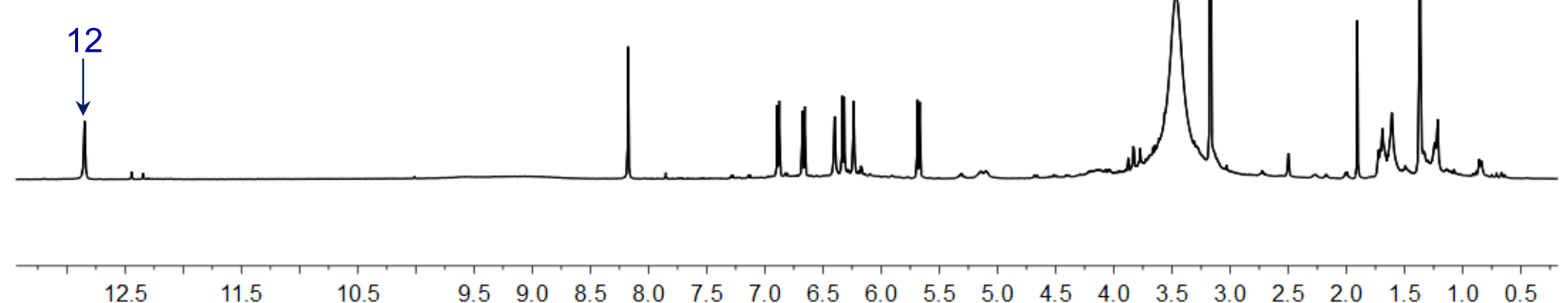

10.5

$\begin{array}{lllllll}9.5 & 9.0 & 8.5 & 8.0 & 7.5 & 7.0 & 6.5\end{array}$

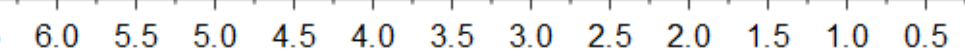

Figure S39. The ${ }^{1} \mathrm{H}$ NMR spectrum of GU-MF-14 in DMSO- $d_{6}$. 


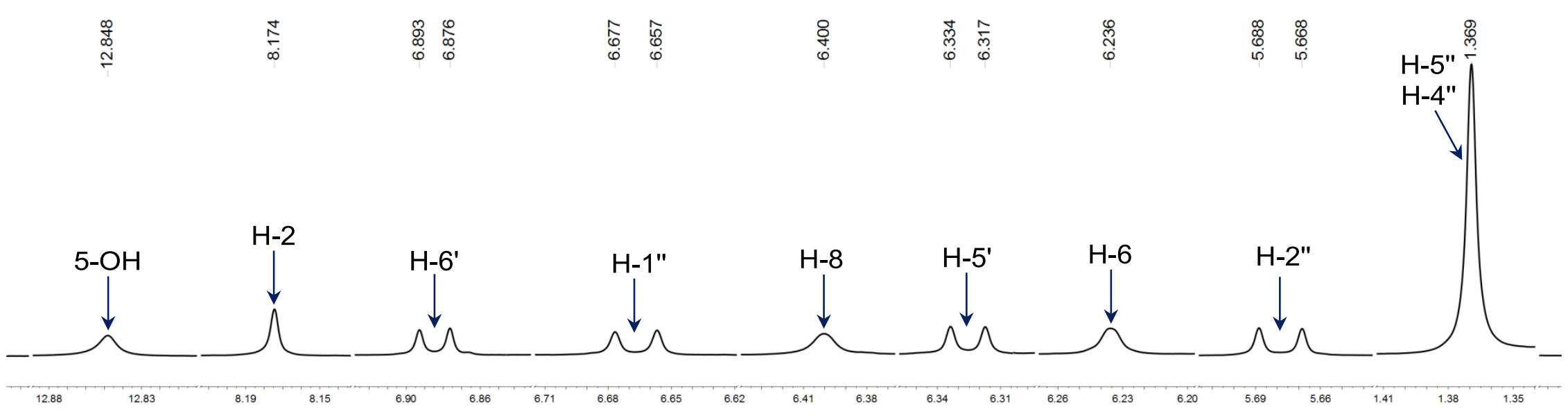

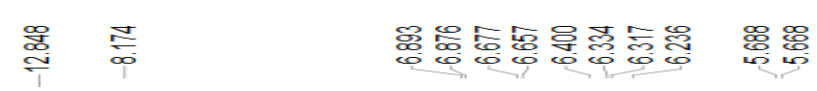
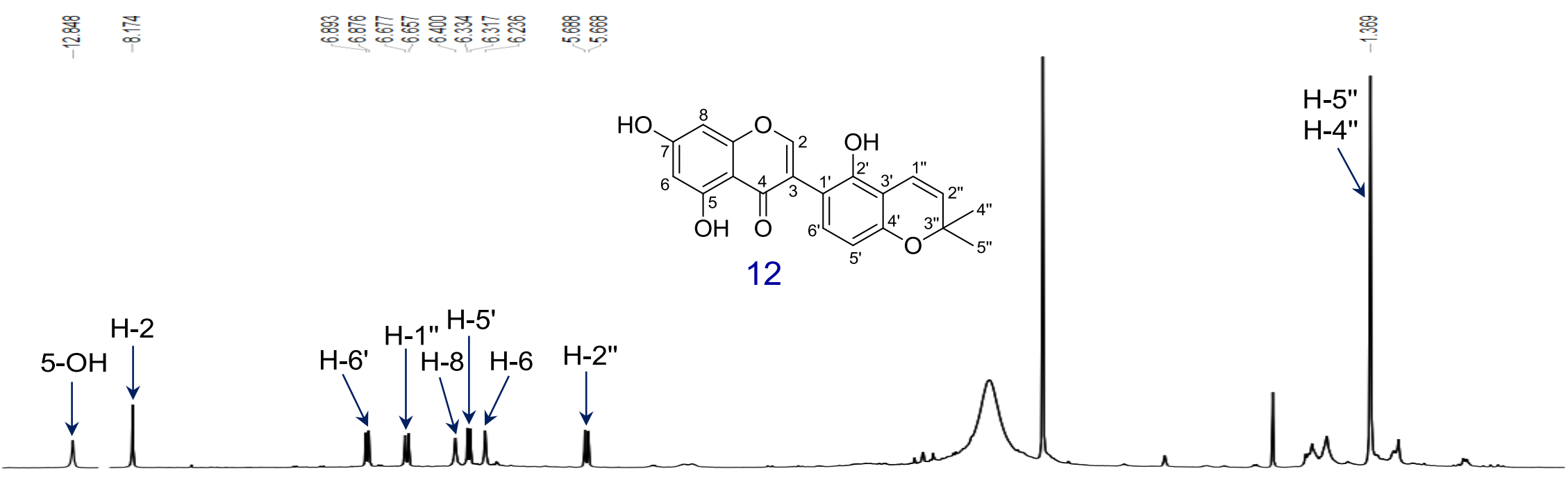

Figure S40. The ${ }^{1} \mathrm{H}^{7.5} \mathrm{NMR}$ spectrum of compound 12 in GU-MF-14 in DMSO- ${ }_{6}$ 


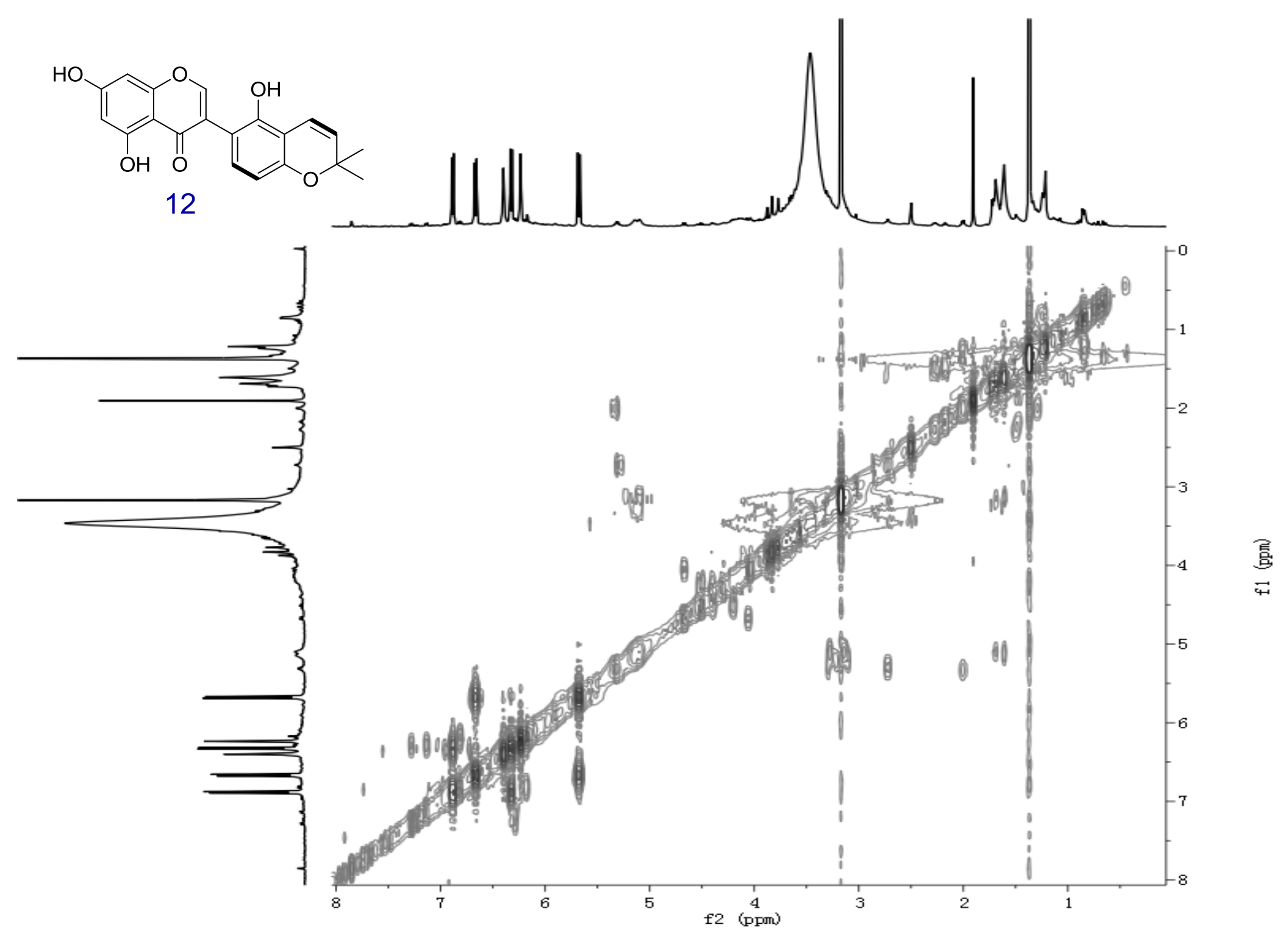

Figure S41. The ${ }^{1} \mathrm{H}-{ }^{1} \mathrm{H}$ COSY spectrum of GU-MF-14 in DMSO- $d_{6}$. 


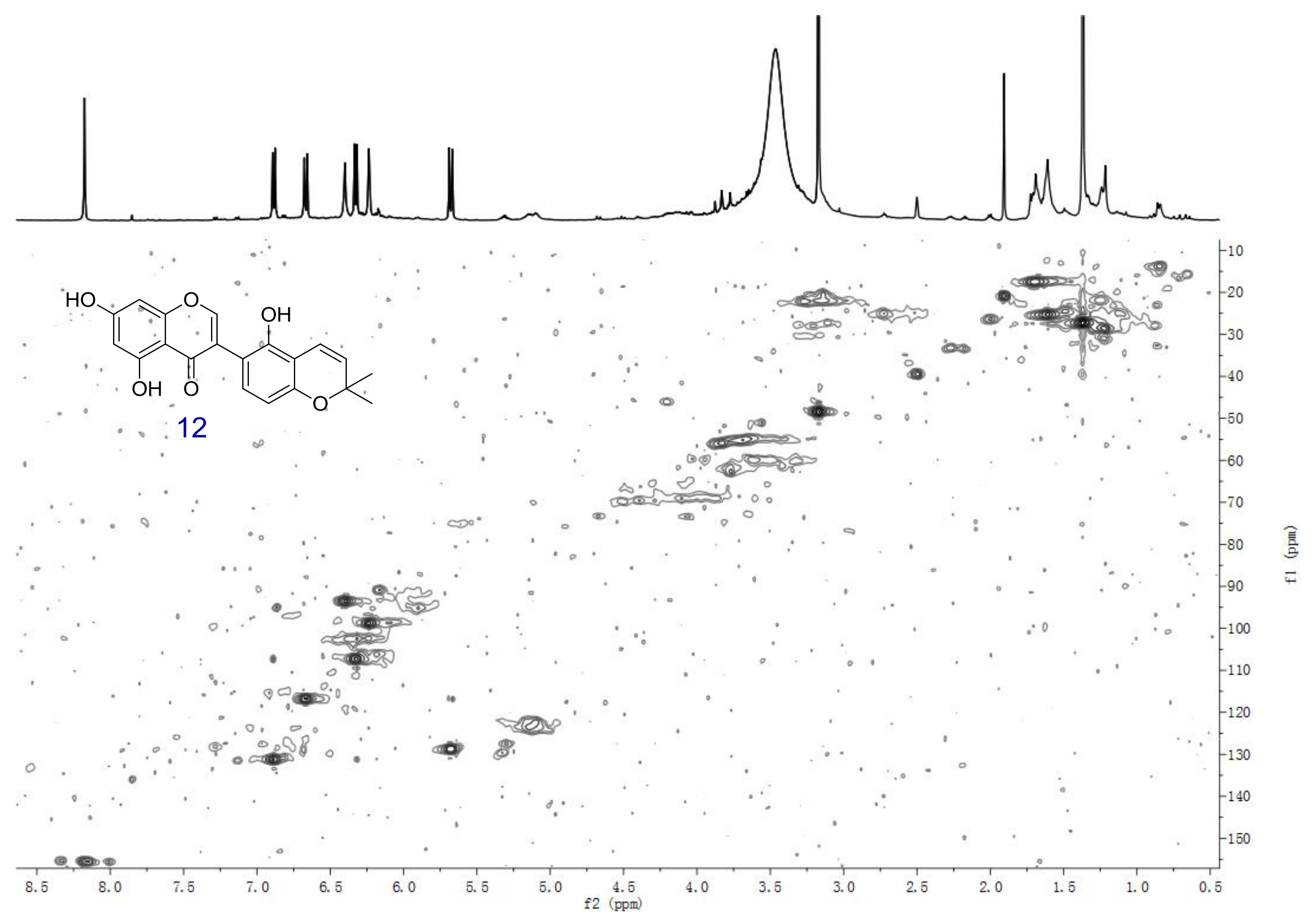

Figure S42. The HSQC spectrum of GU-MF-14 in DMSO- $d_{6}$. 


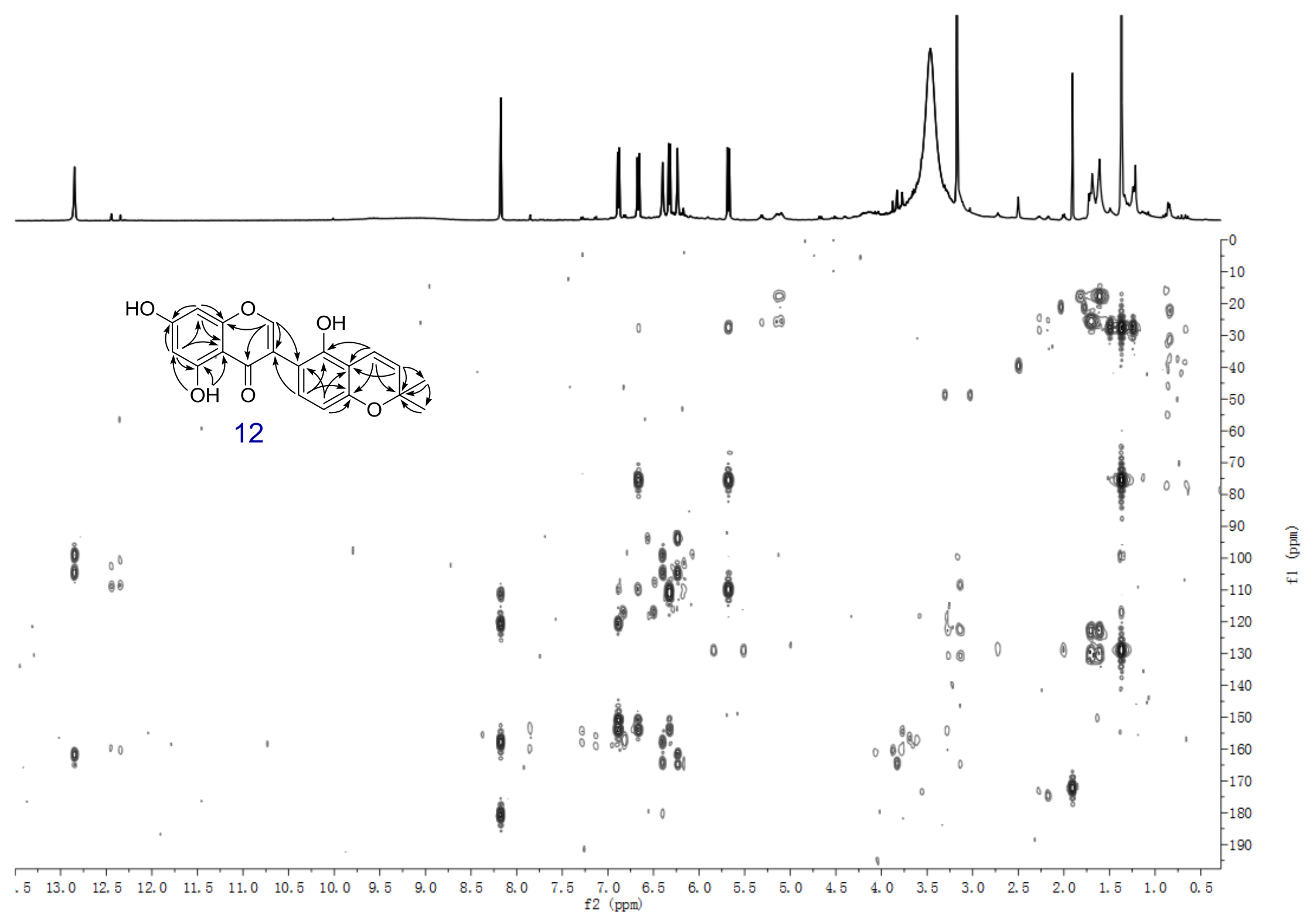

Figure S43. The HMBC spectrum of GU-MF-14 in DMSO- $d_{6}$. 

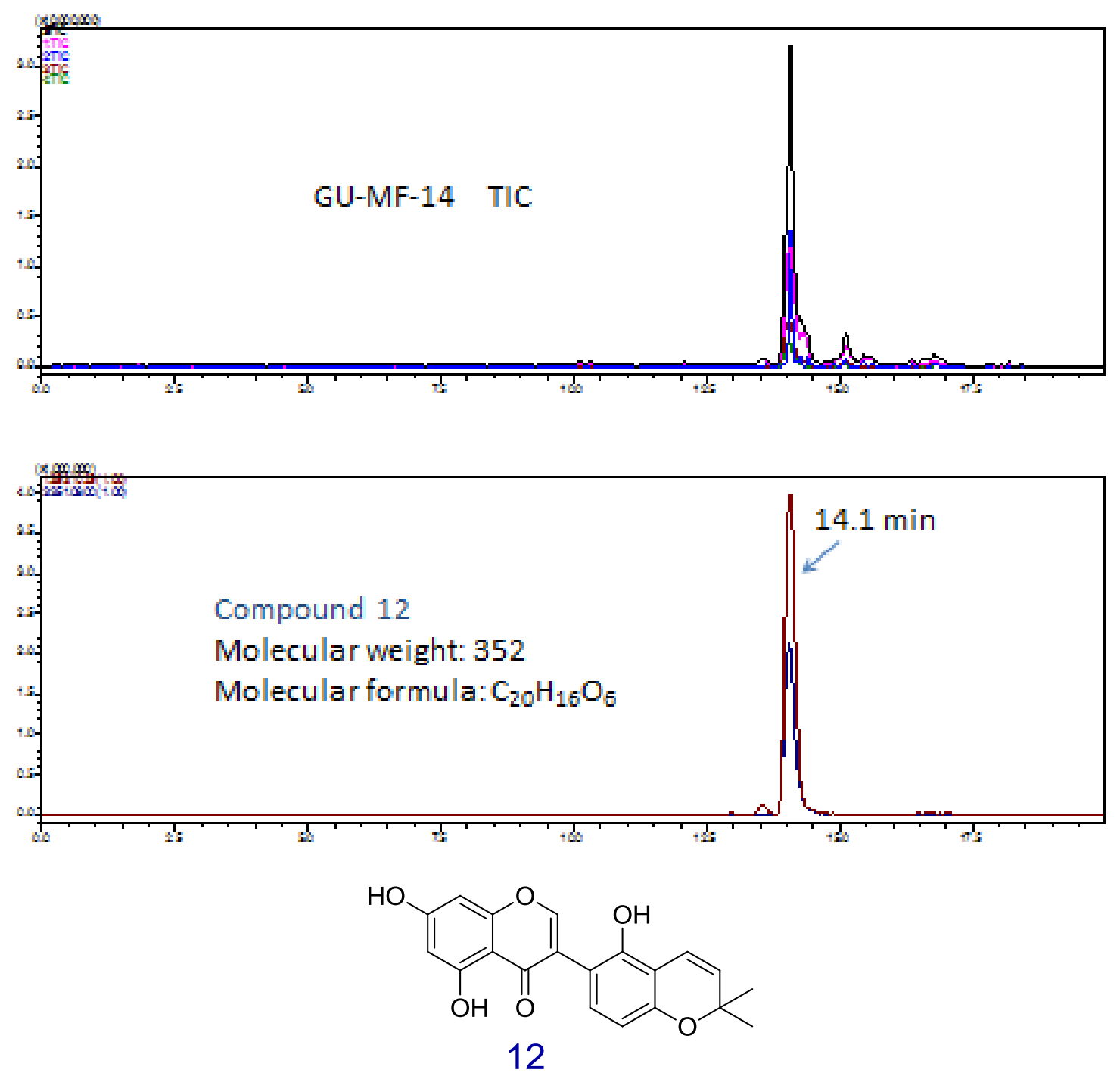

Figure S44. The IT-TOF TIC and mass chromatogram of GU-MF-14. 

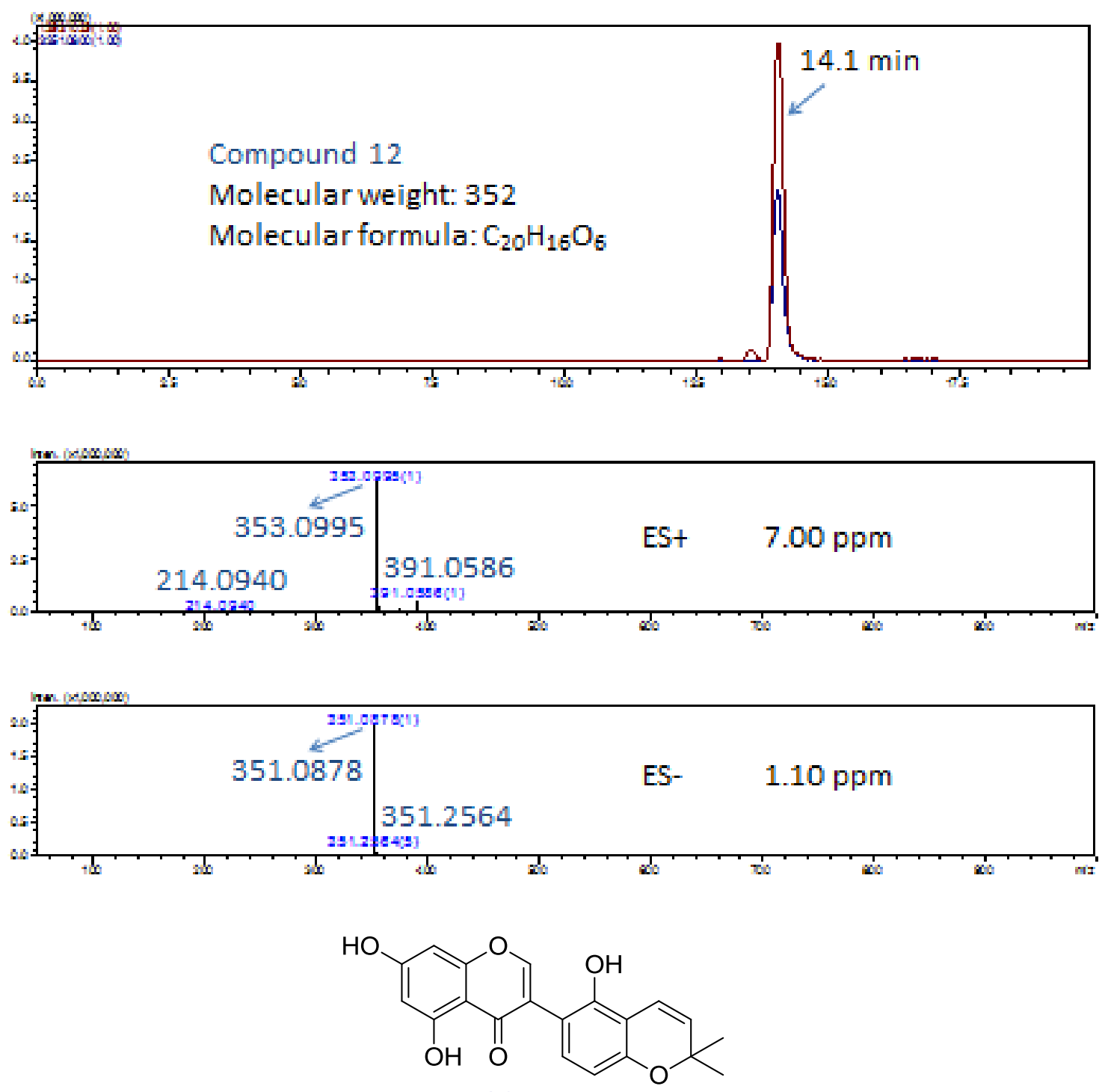

12

Figure S45. The (+)-HRESIMS and (-)-HRESIMS spectra of compound 12 in GU-MF-14 with extracted ions (positive and negative) for $m / z 353$ and 351, respectively. 


\section{彭品品}

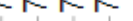

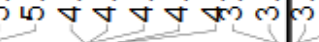

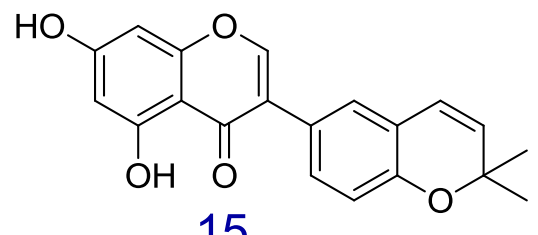

15
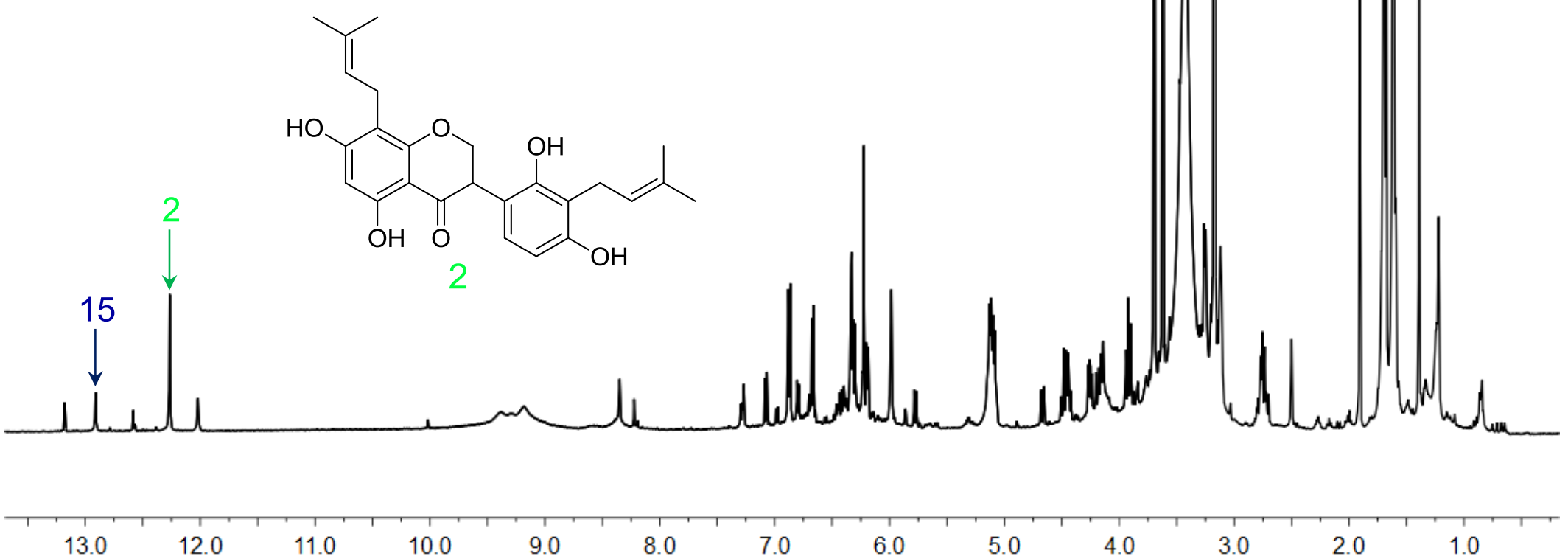

Figure S46. The ${ }^{1} \mathrm{H}$ NMR spectrum of GU-MF-15 in DMSO- $d_{6}$. 


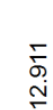

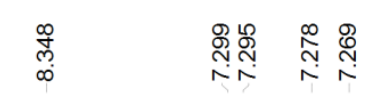

$\underset{\substack{0 \\ 0 \\ 0}}{0}$

$\underset{\substack{\infty \\ \hdashline}}{\stackrel{\infty}{0}}$

$\underset{\substack{\mathscr{g} \\ 6}}{6}$

$\stackrel{\infty}{\frac{0}{6}}$

ब్

$\underset{\substack{\tilde{N} \\ \text { d }}}{ }$

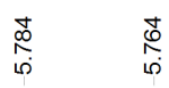

$5-\mathrm{OH}$

H-2
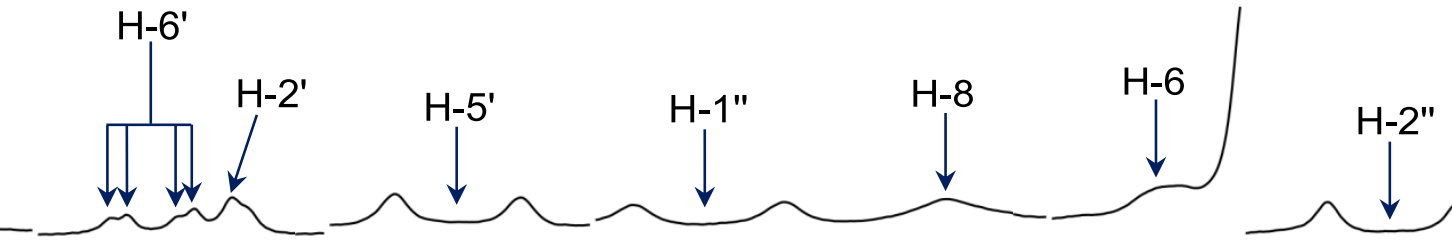

$\mathrm{H}-5 "$
$\mathrm{H}-4 "$

12.93
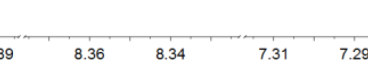

产 $\quad$ 莙

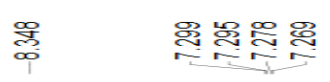

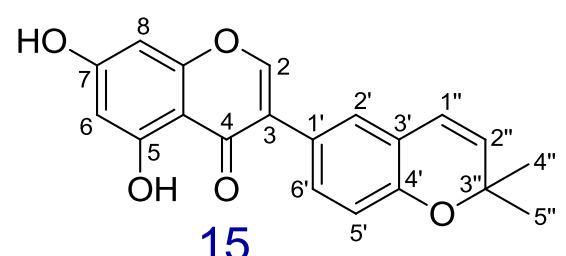

15
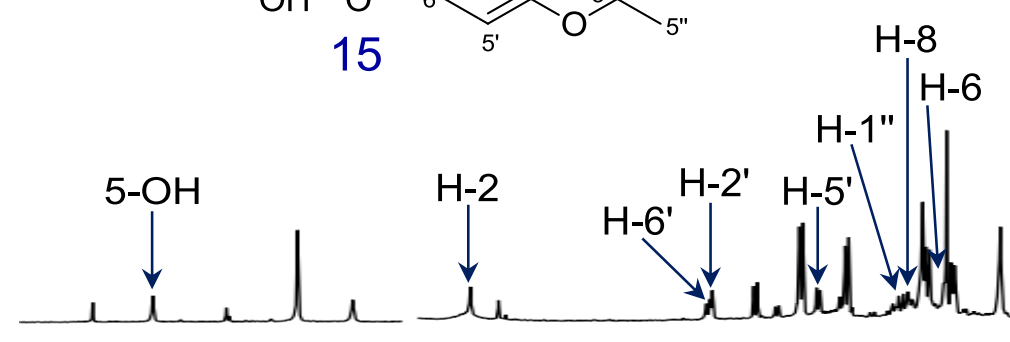

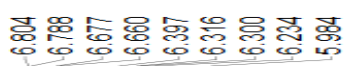

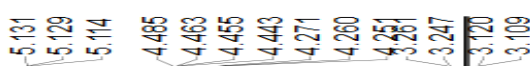

5.790

5.775

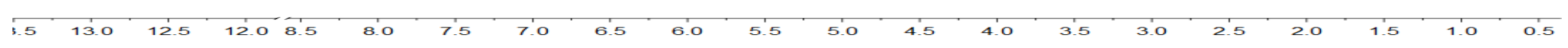

Figure S47. The ${ }^{1} \mathrm{H}$ NMR spectrum of compound 15 in GU-MF-15 in DMSO- $d_{6}$.

73 


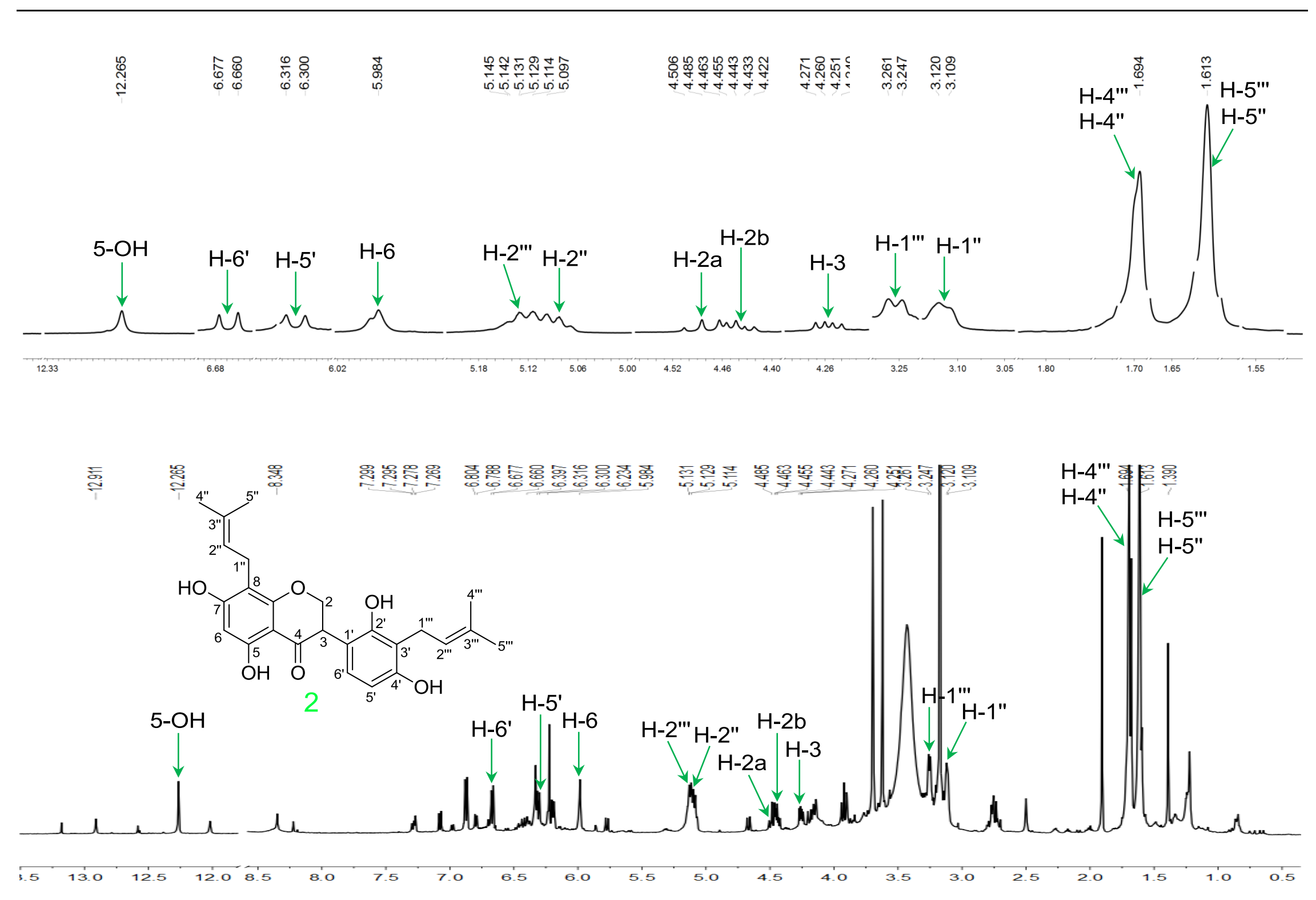

Figure S48. The ${ }^{1} \mathrm{H}$ NMR spectrum of compound 2 in GU-MF-15 in DMSO- $d_{6}$. 


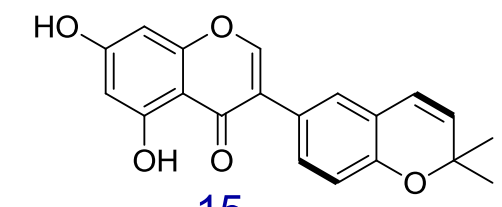

15
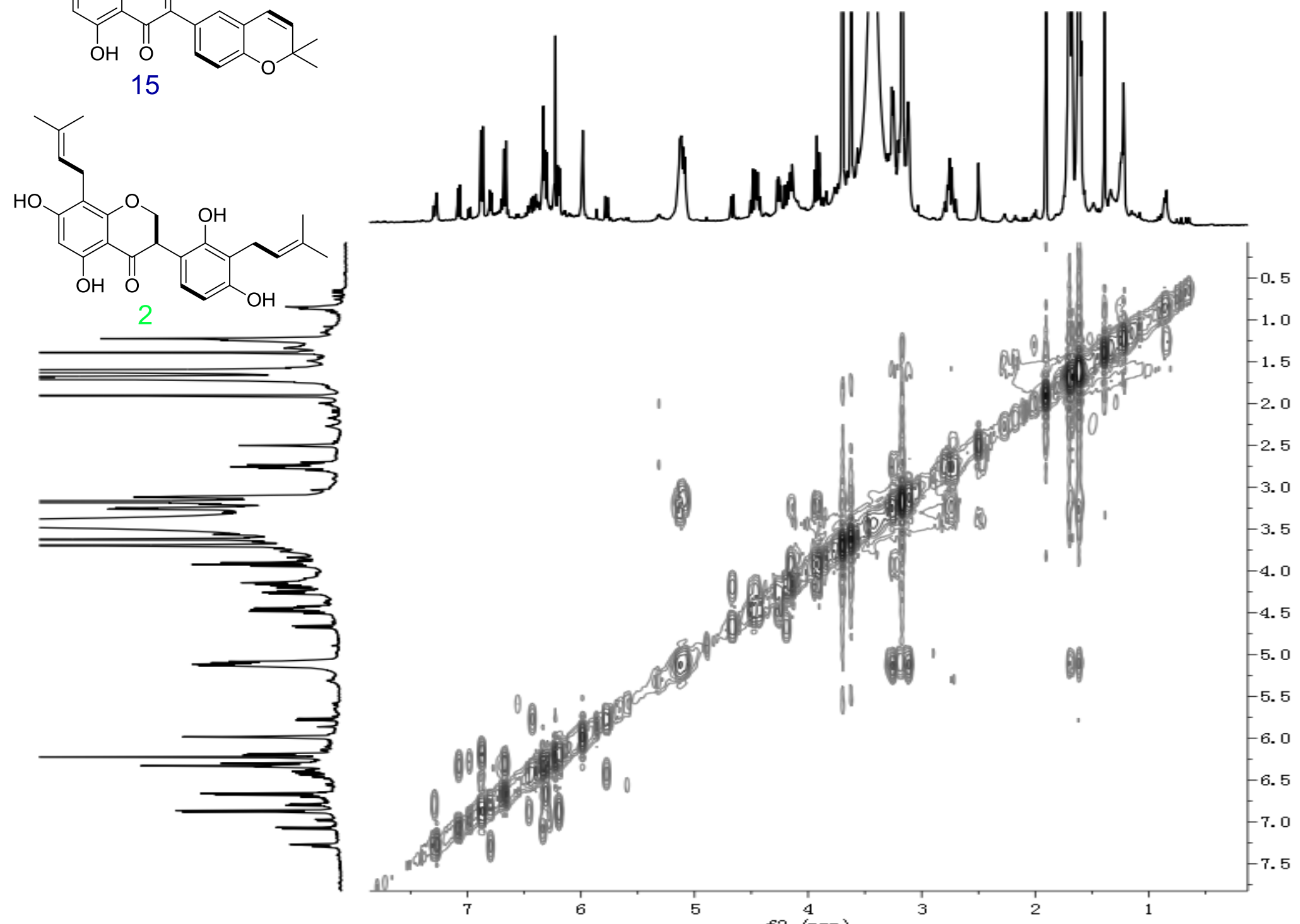

Figure S49. The ${ }^{1} \mathrm{H}-{ }^{1} \mathrm{H}$ COSY spectrum of GU-MF-15 in DMSO- $d_{6}$. 


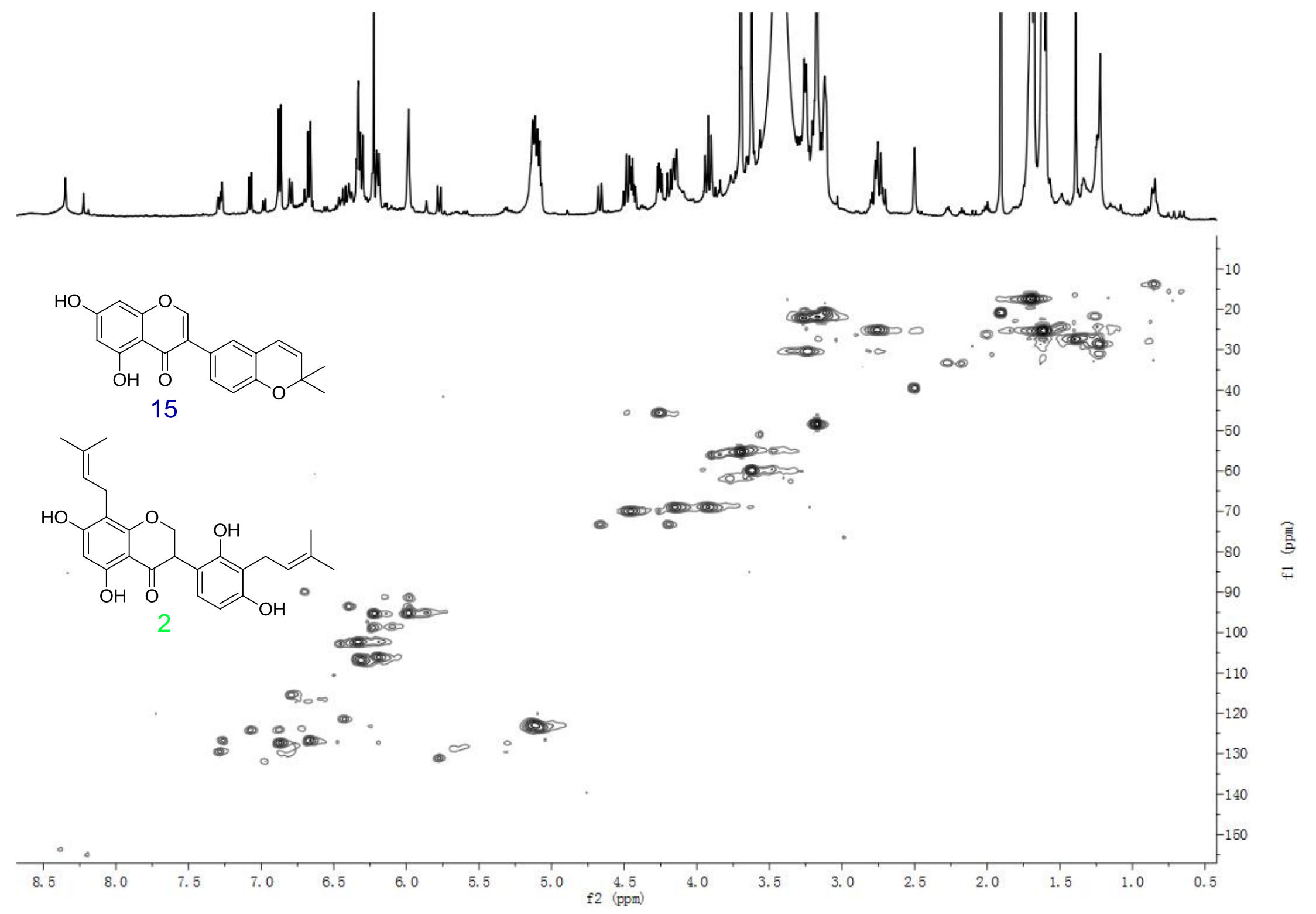

Figure S50. The HSQC spectrum of GU-MF-15 in DMSO- $d_{6}$. 


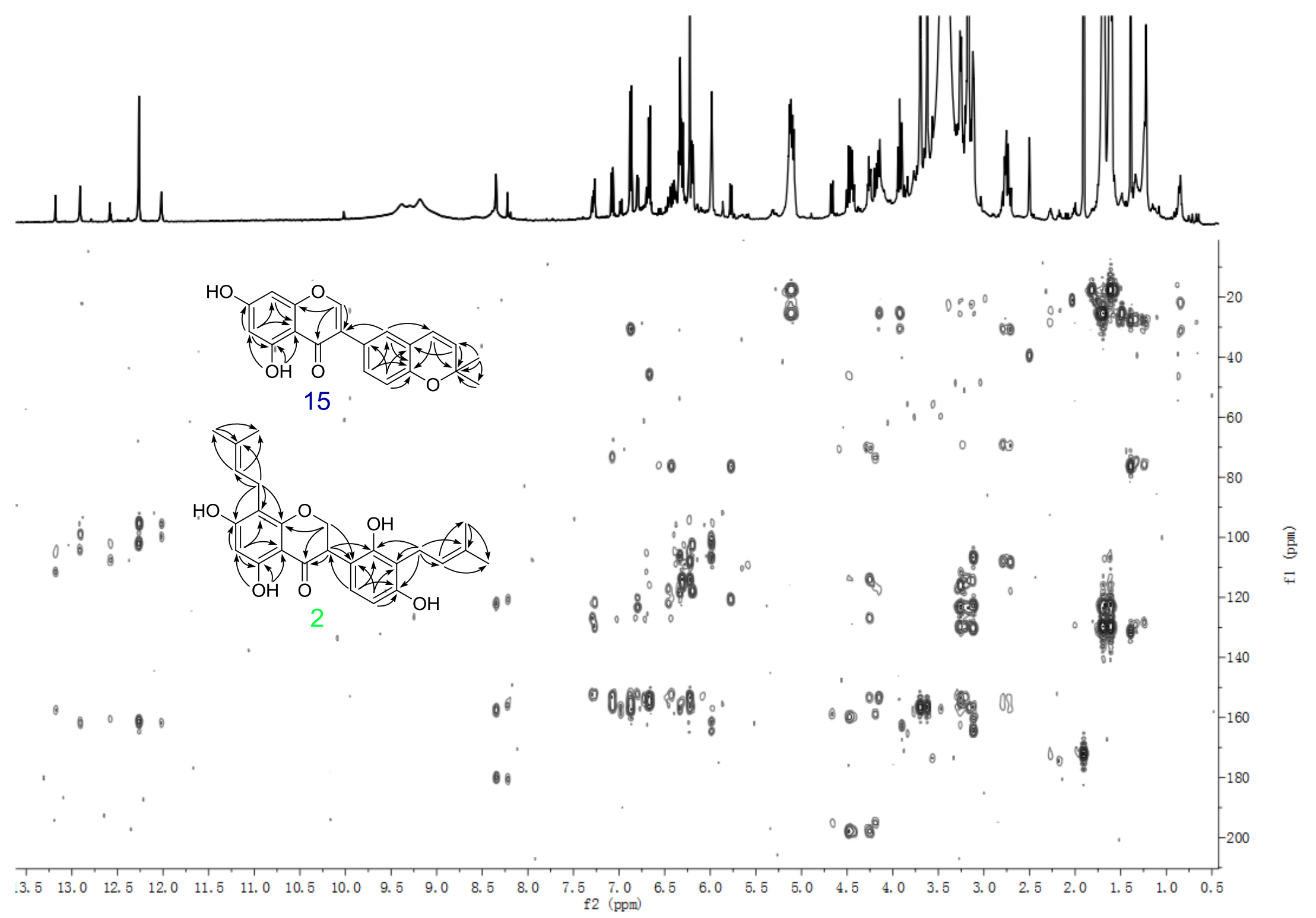

Figure S51. The HMBC spectrum of GU-MF-15 in DMSO- $d_{6}$. 

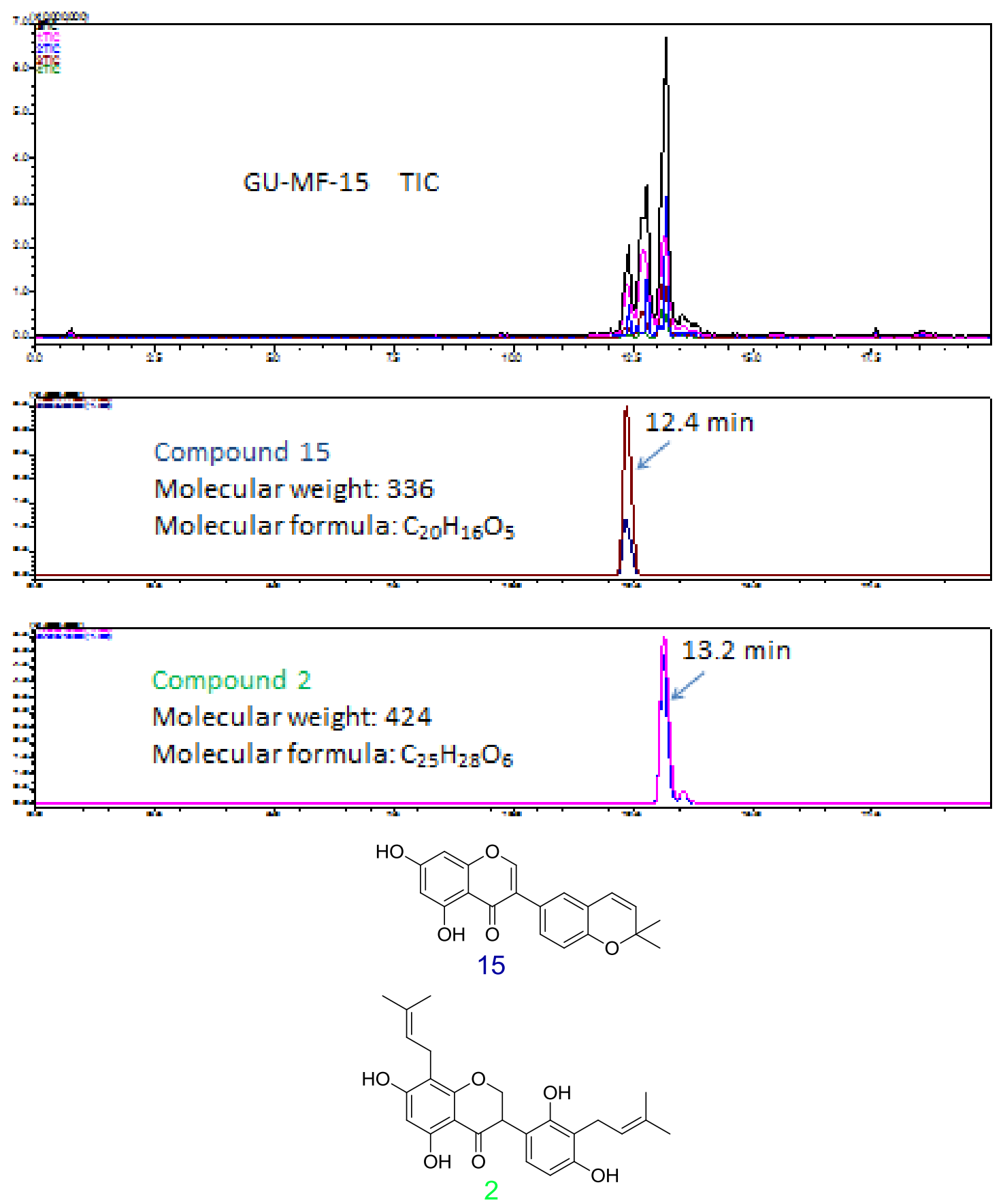

Figure S52. The IT-TOF TIC and mass chromatogram of GU-MF-15. 

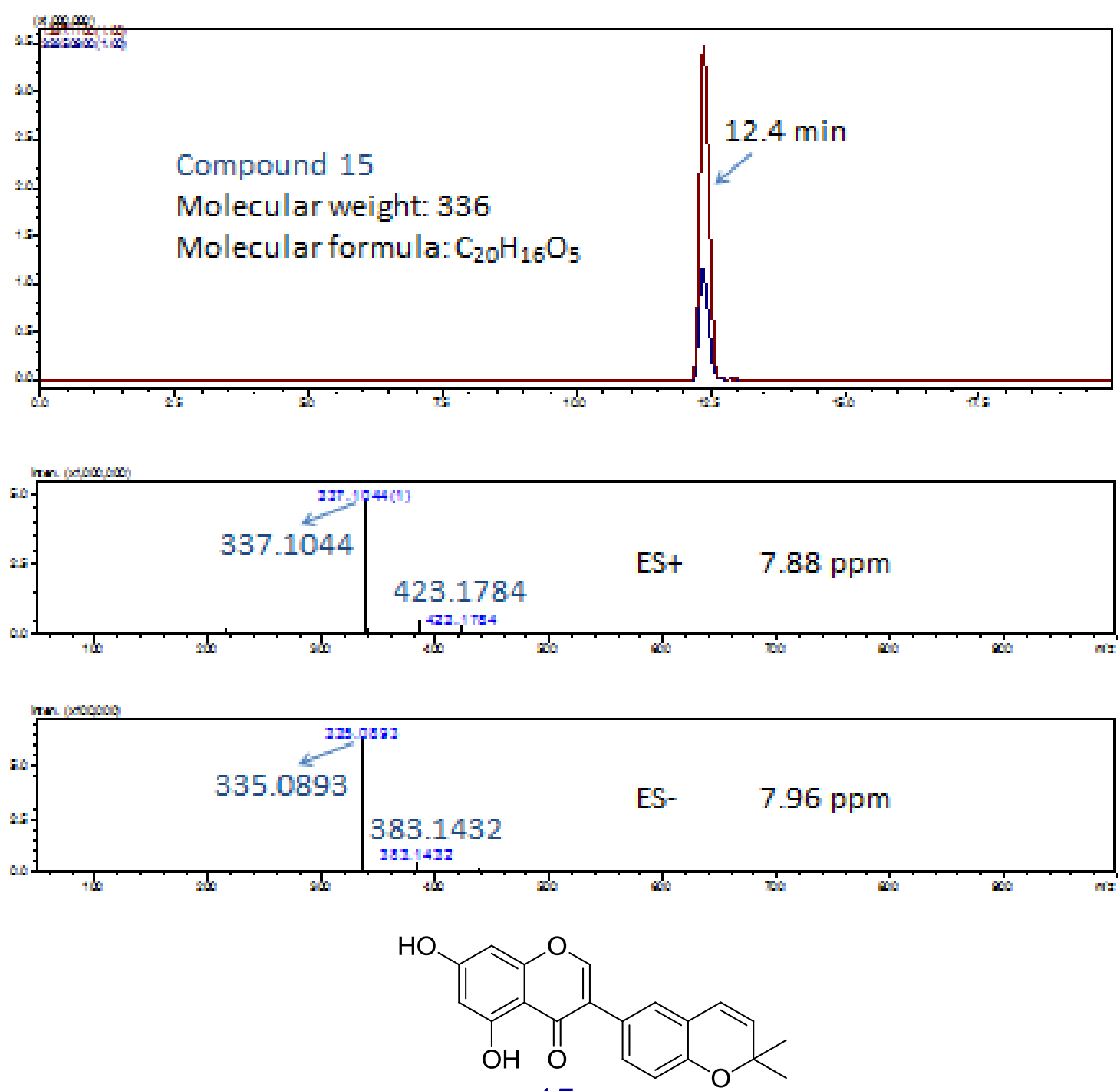

15

Figure S53. The (+)-HRESIMS and (-)-HRESIMS spectra of compound 15 in GU-MF-15 with extracted ions (positive and negative) for $\mathrm{m} / \mathrm{z} 337$ and 335, respectively. 

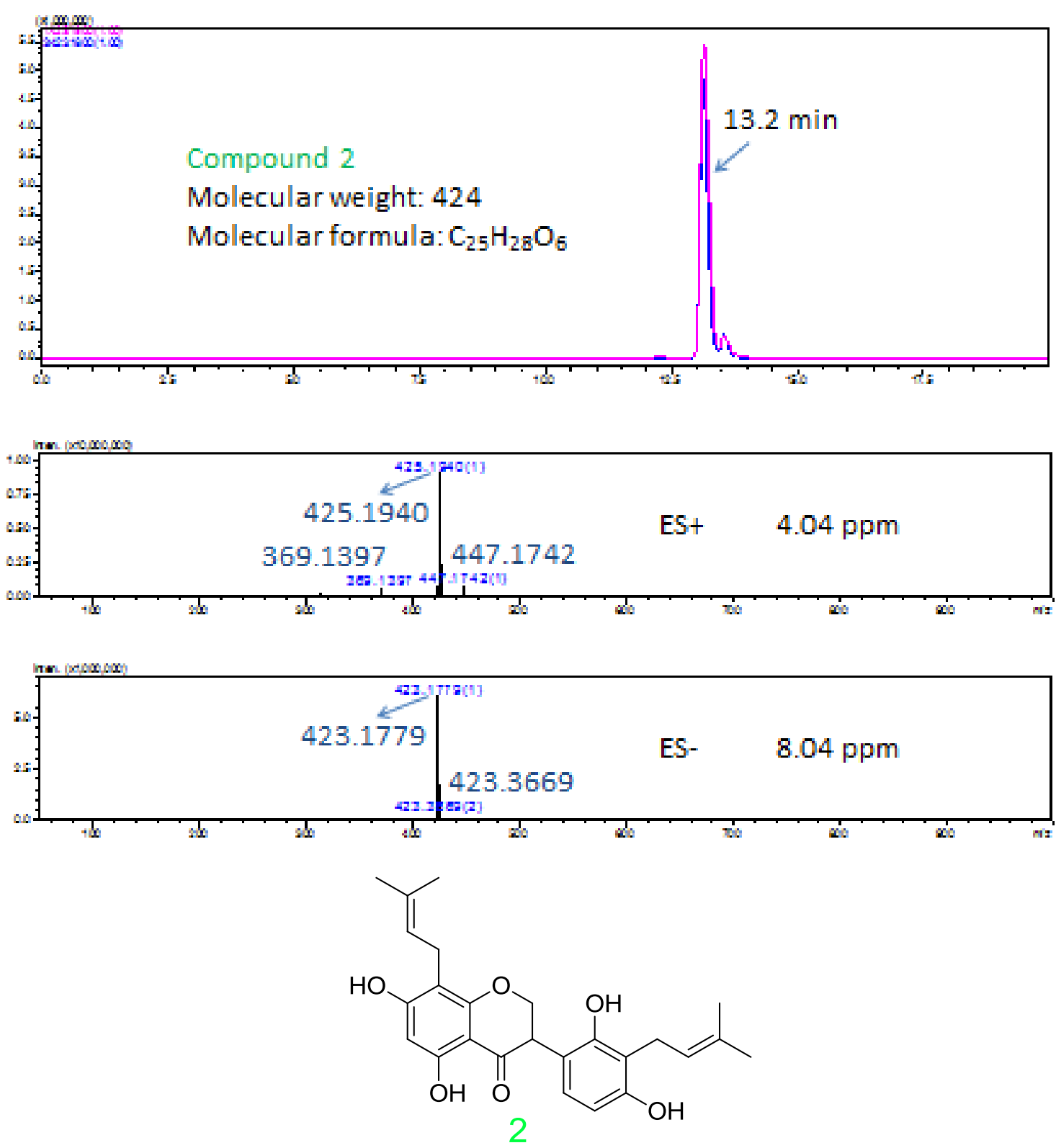

Figure S54. The (+)-HRESIMS and (-)-HRESIMS spectra of compound 2 in GU-MF-15 with extracted ions (positive and negative) for $m / z, 425$ and 423, respectively. 


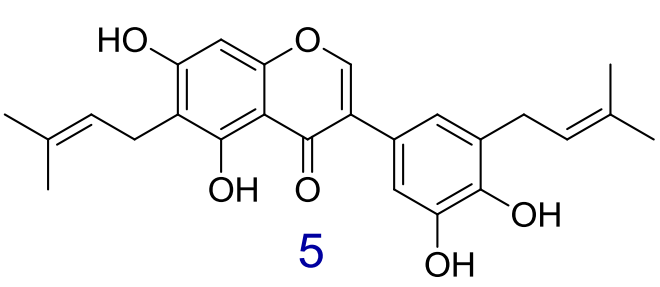

5

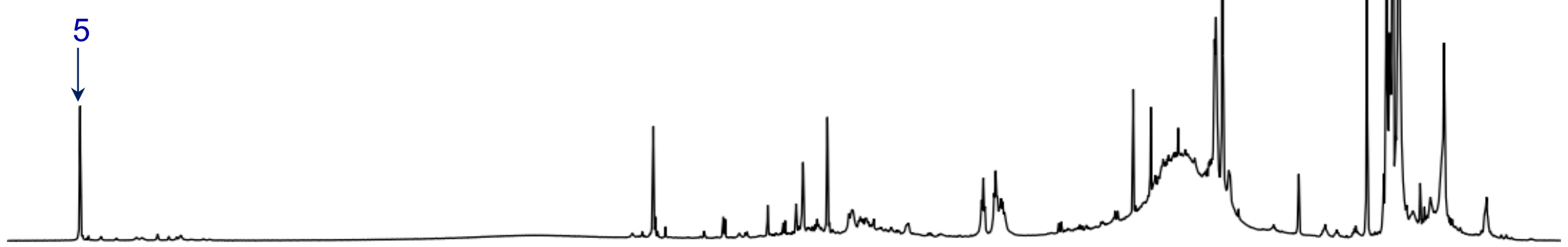

13.0

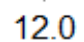

11.0

10.0

9.0

8.0

7.0

$6.0 \quad 5.0$

4.0

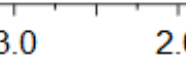

2.0

1.0

Figure S55. The ${ }^{1} \mathrm{H}$ NMR spectrum of GU-MF-16 in DMSO- $d_{6}$. 


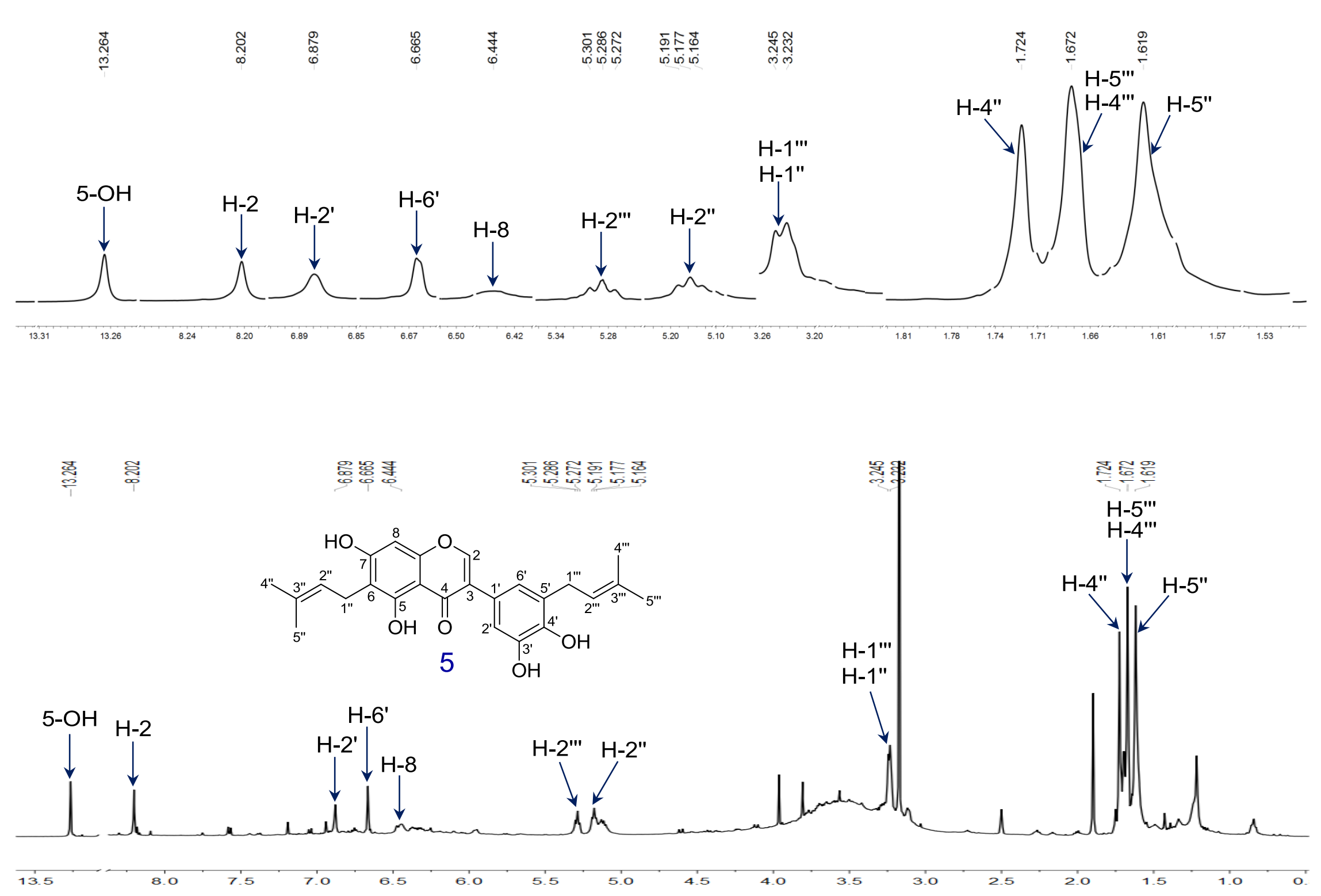

Figure S56. The ${ }^{1} \mathrm{H}$ NMR spectrum of compound 5 in GU-MF-16 in DMSO- $d_{6}$. 


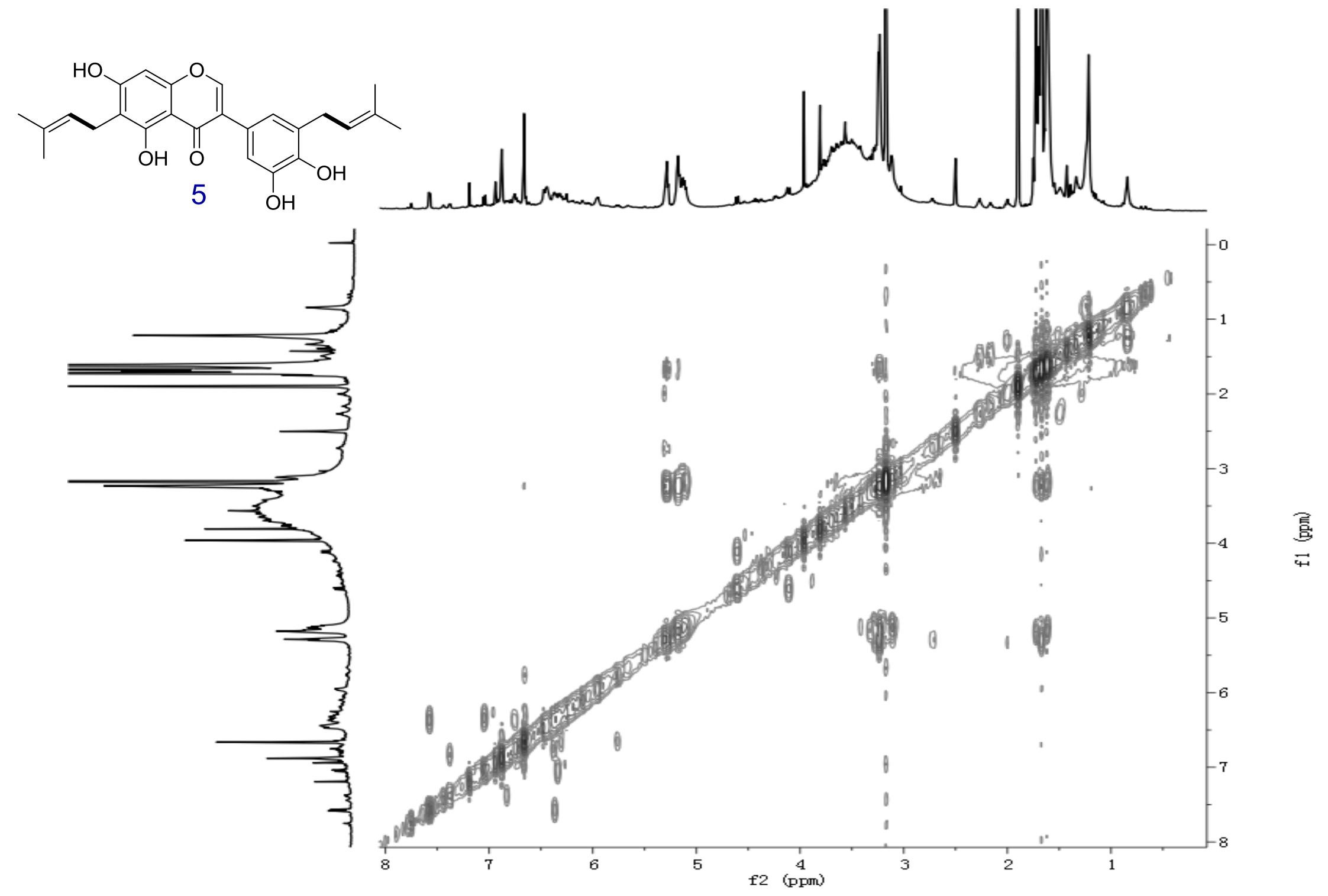

Figure S57. The ${ }^{1} \mathrm{H}-{ }^{1} \mathrm{H}$ COSY spectrum of GU-MF-16 in DMSO- $d_{6}$. 


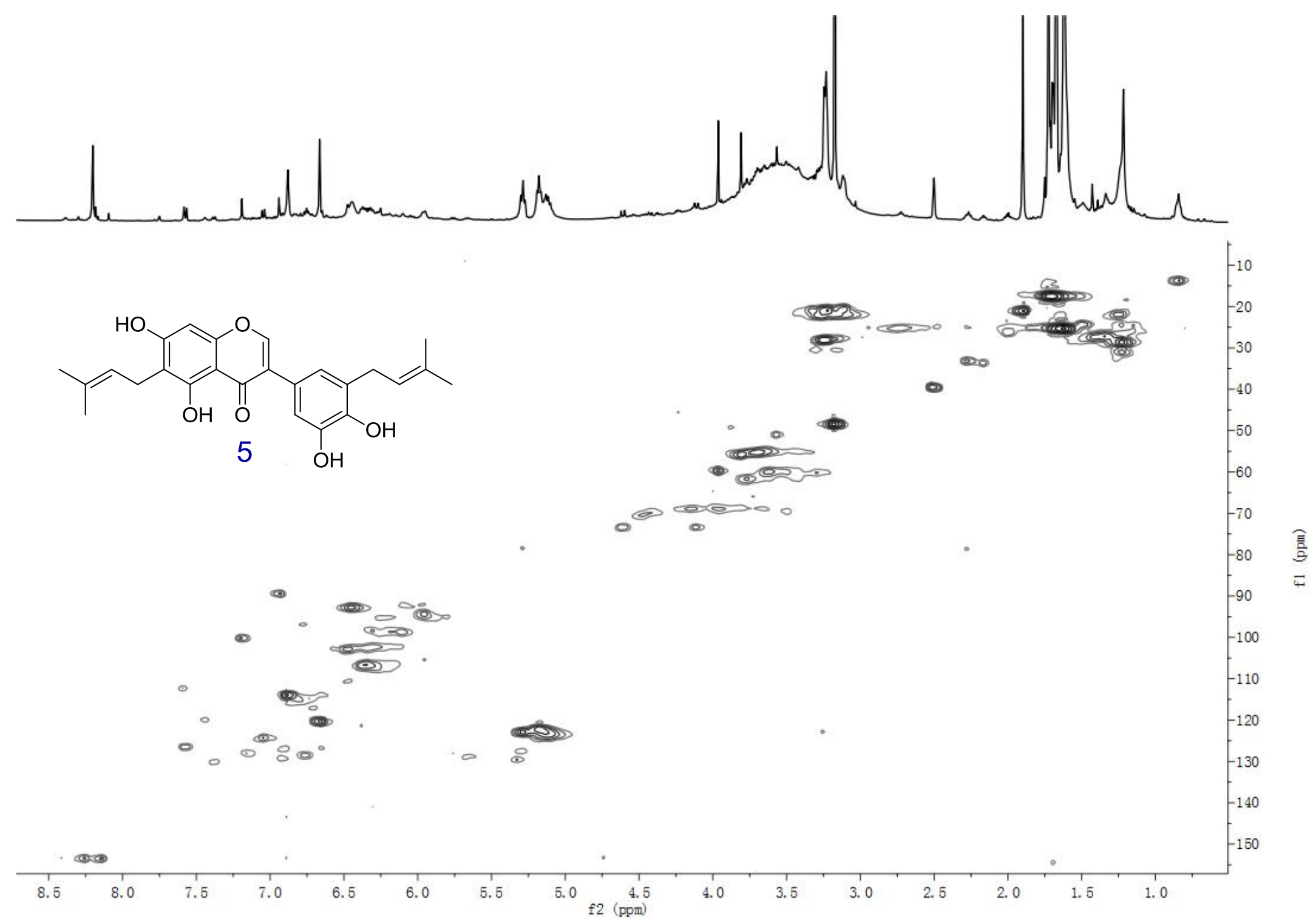

Figure S58. The HSQC spectrum of GU-MF-16 in DMSO- $d_{6}$. 


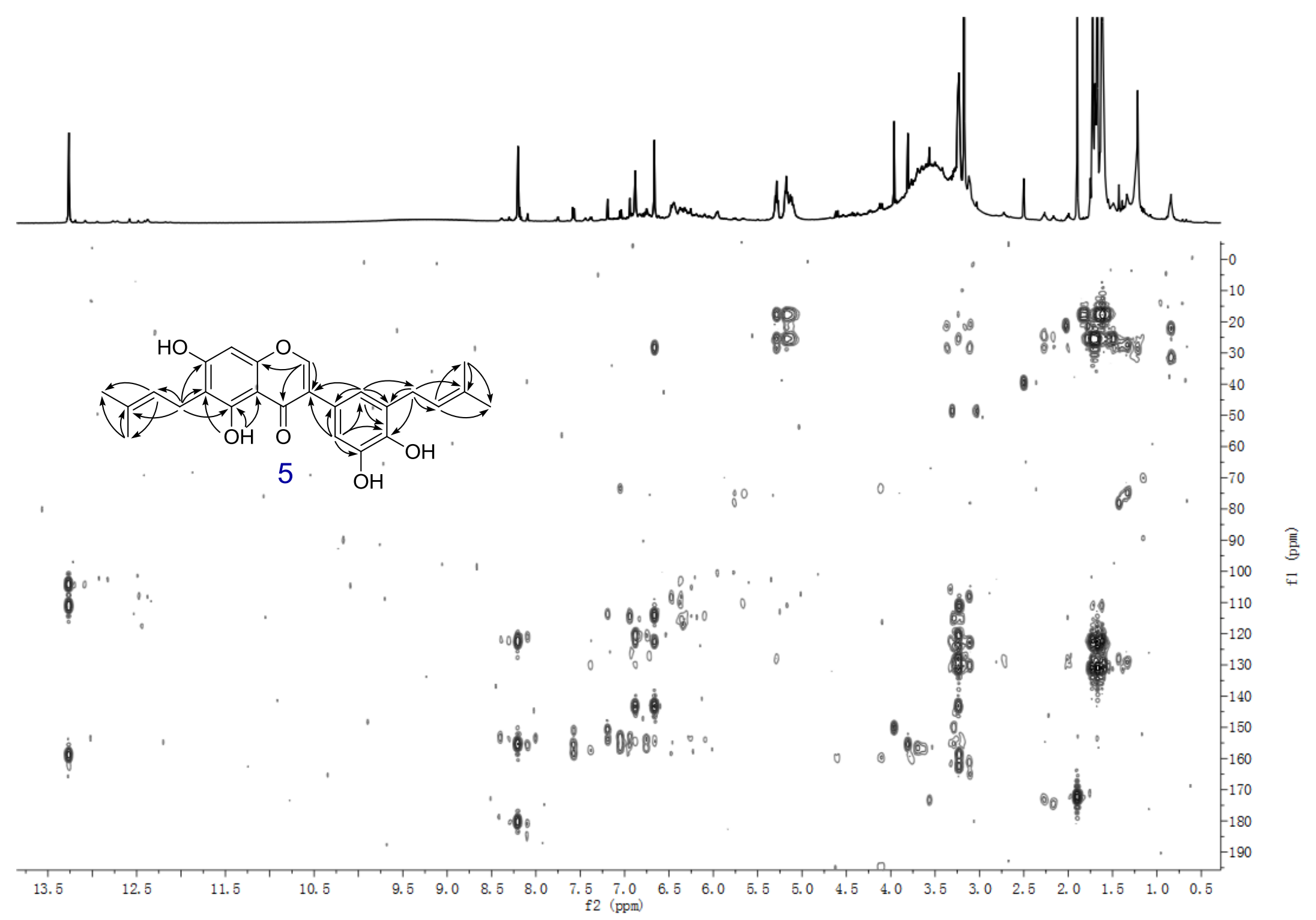

Figure S59. The HMBC spectrum of GU-MF-16 in DMSO- $d_{6}$. 

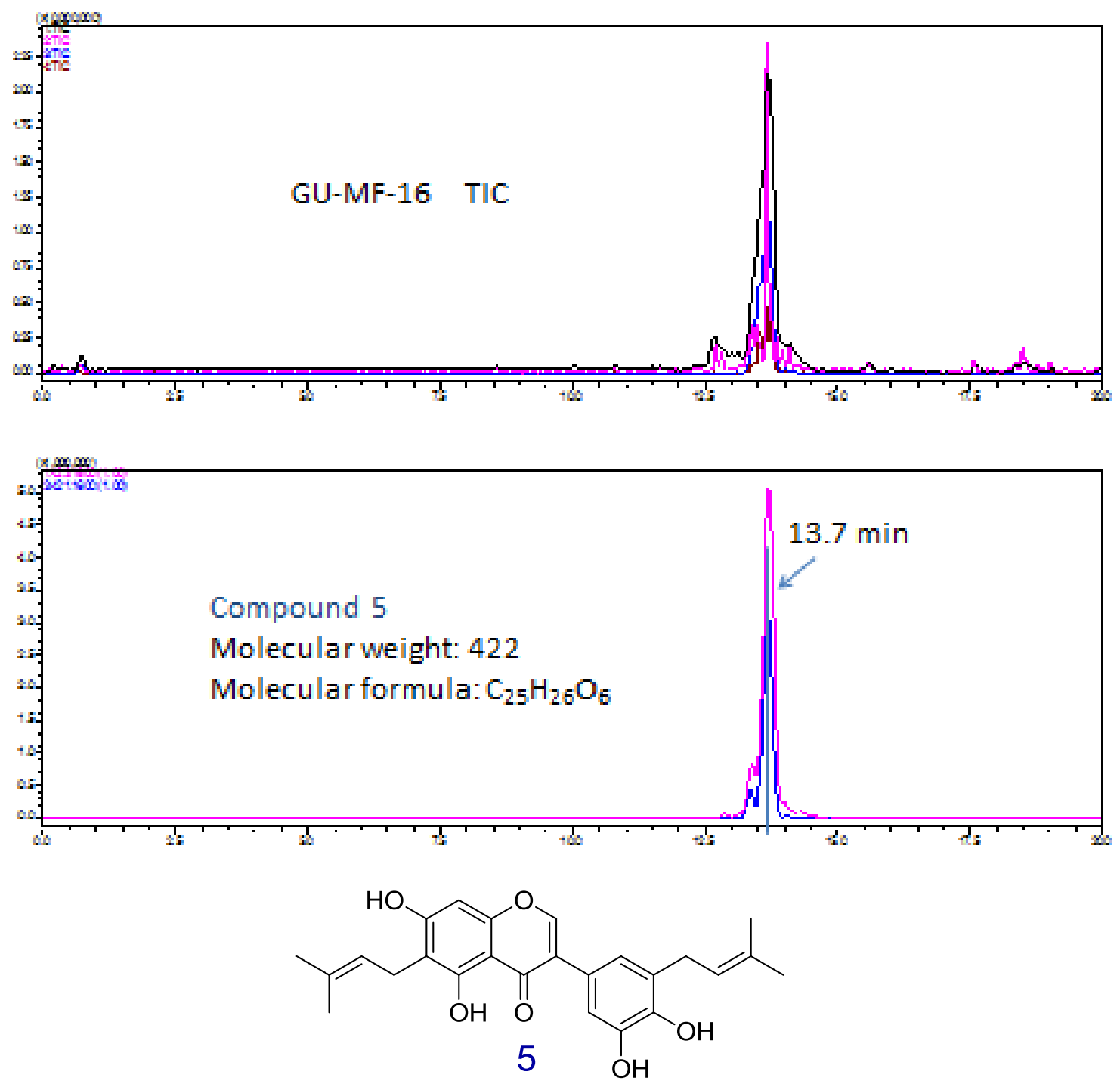

Figure S60. The IT-TOF TIC and mass chromatogram of GU-MF-16. 

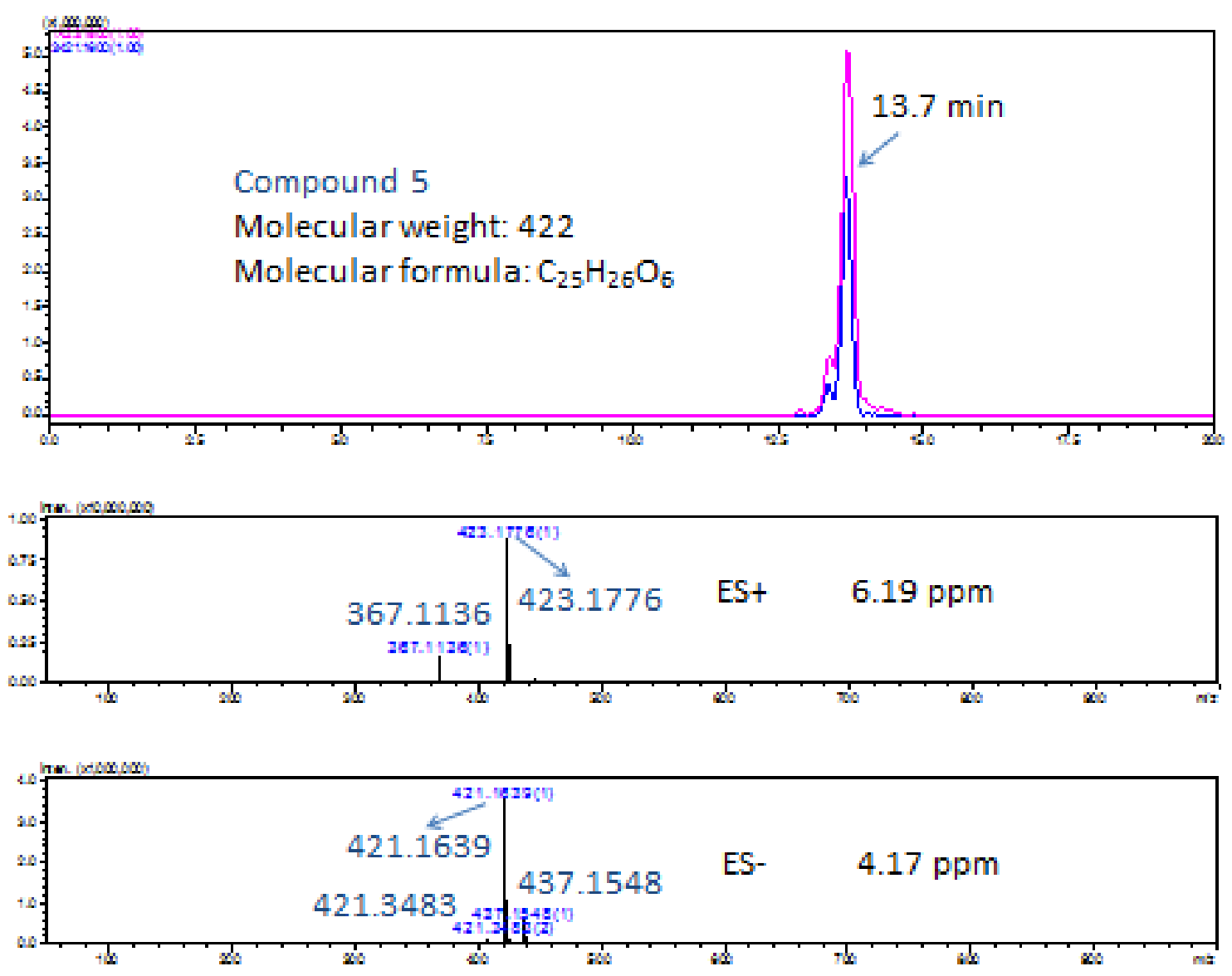<smiles>CC(C)=CCc1cc(-c2coc3cc(O)c(CC=C(C)C)c(O)c3c2=O)cc(O)c1O</smiles>

Figure S61. The (+)-HRESIMS and (-)-HRESIMS spectra of compound 5 in GU-MF-16 with extracted ions (positive and negative) for $m / z, 423$ and 421, respectively. 

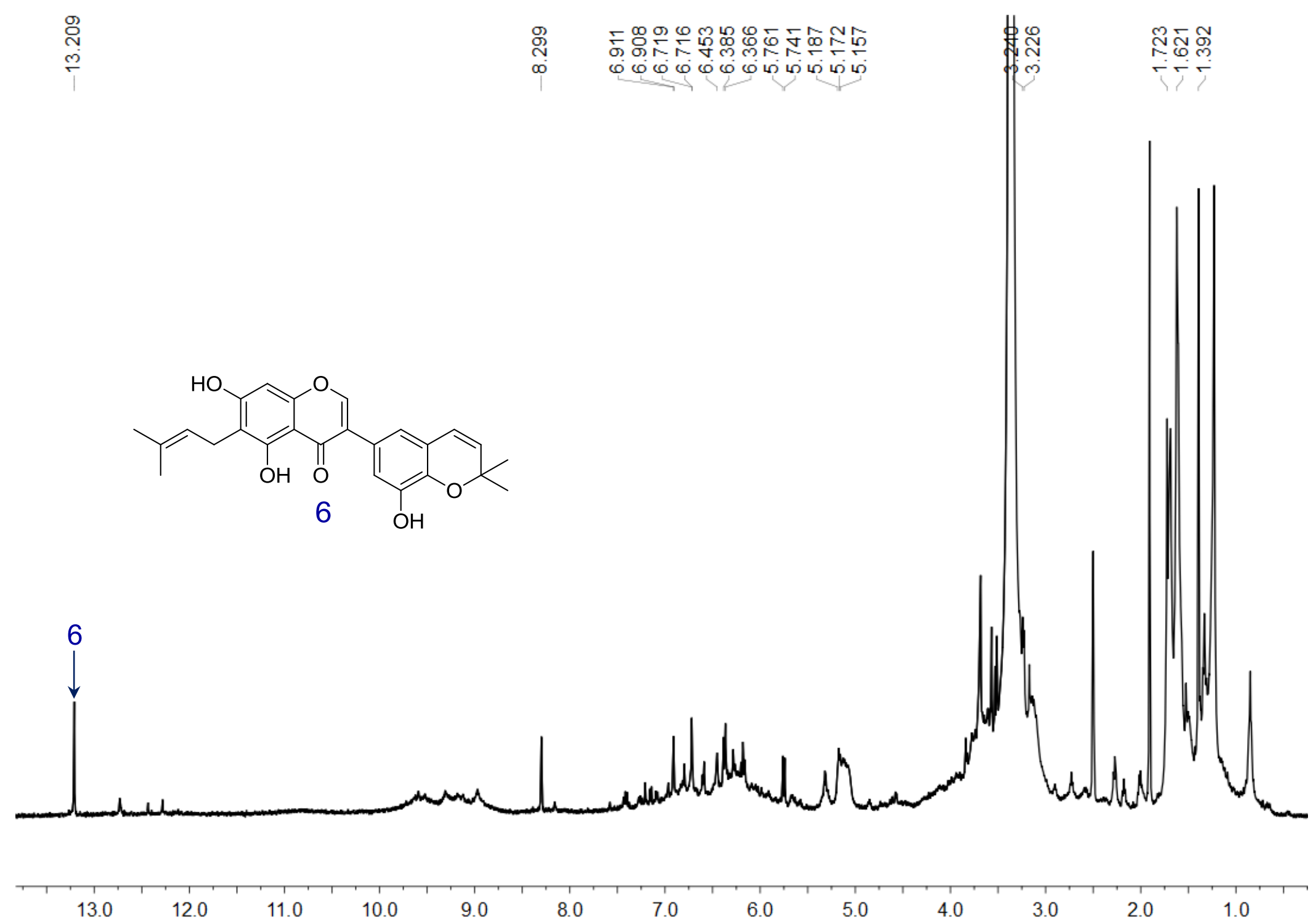

Figure S62. The ${ }^{1} \mathrm{H}$ NMR spectrum of GU-MF-17 in DMSO- $d_{6}$. 

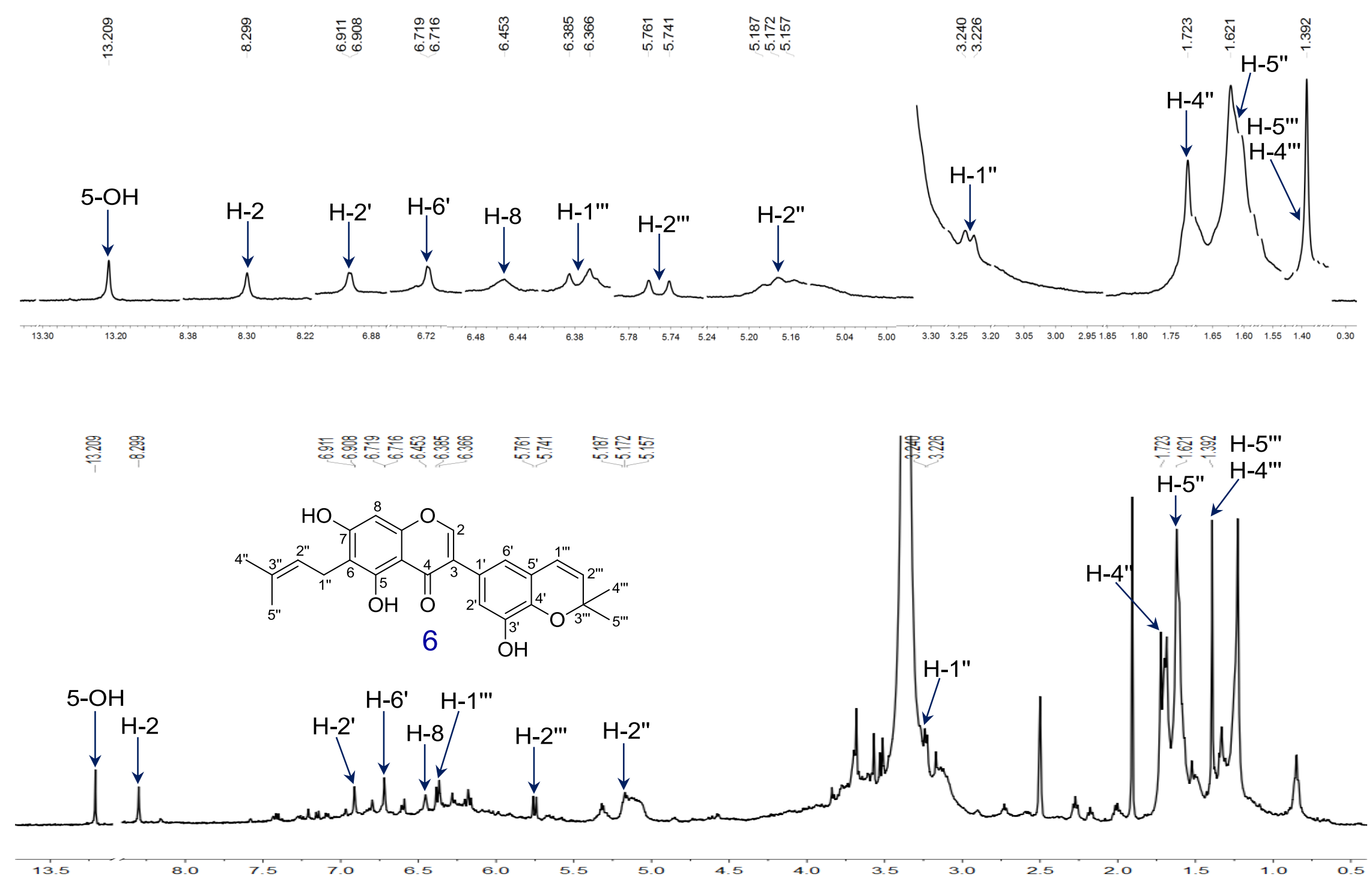

Figure S63. The ${ }^{1} \mathrm{H}$ NMR spectrum of compound 6 in GU-MF-17 in DMSO- $d_{6}$. 


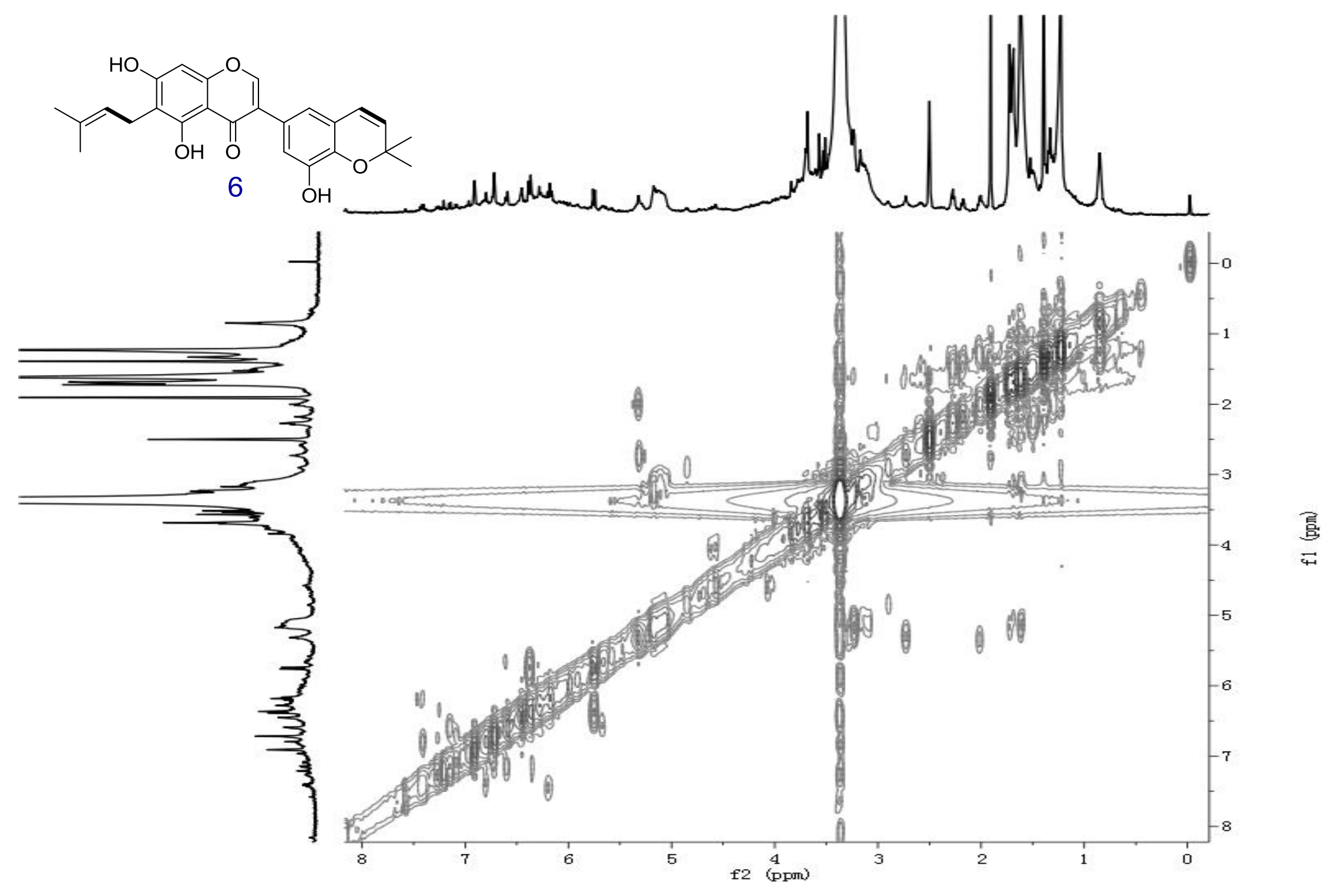

Figure S64. The ${ }^{1} \mathrm{H}-{ }^{1} \mathrm{H}$ COSY spectrum of GU-MF-17 in DMSO- $d_{6}$. 


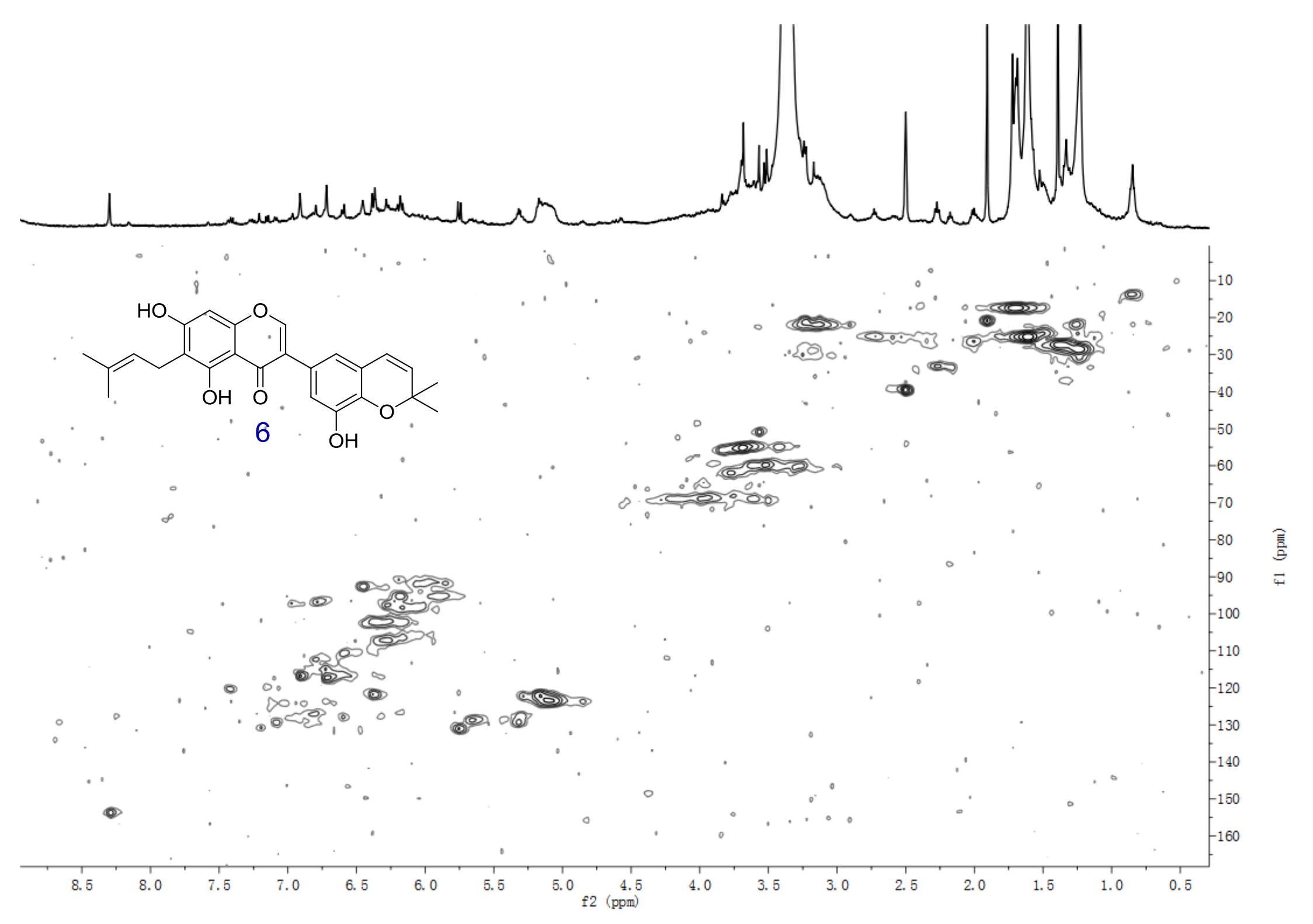

Figure S65. The HSQC spectrum of GU-MF-17 in DMSO- $d_{6}$. 


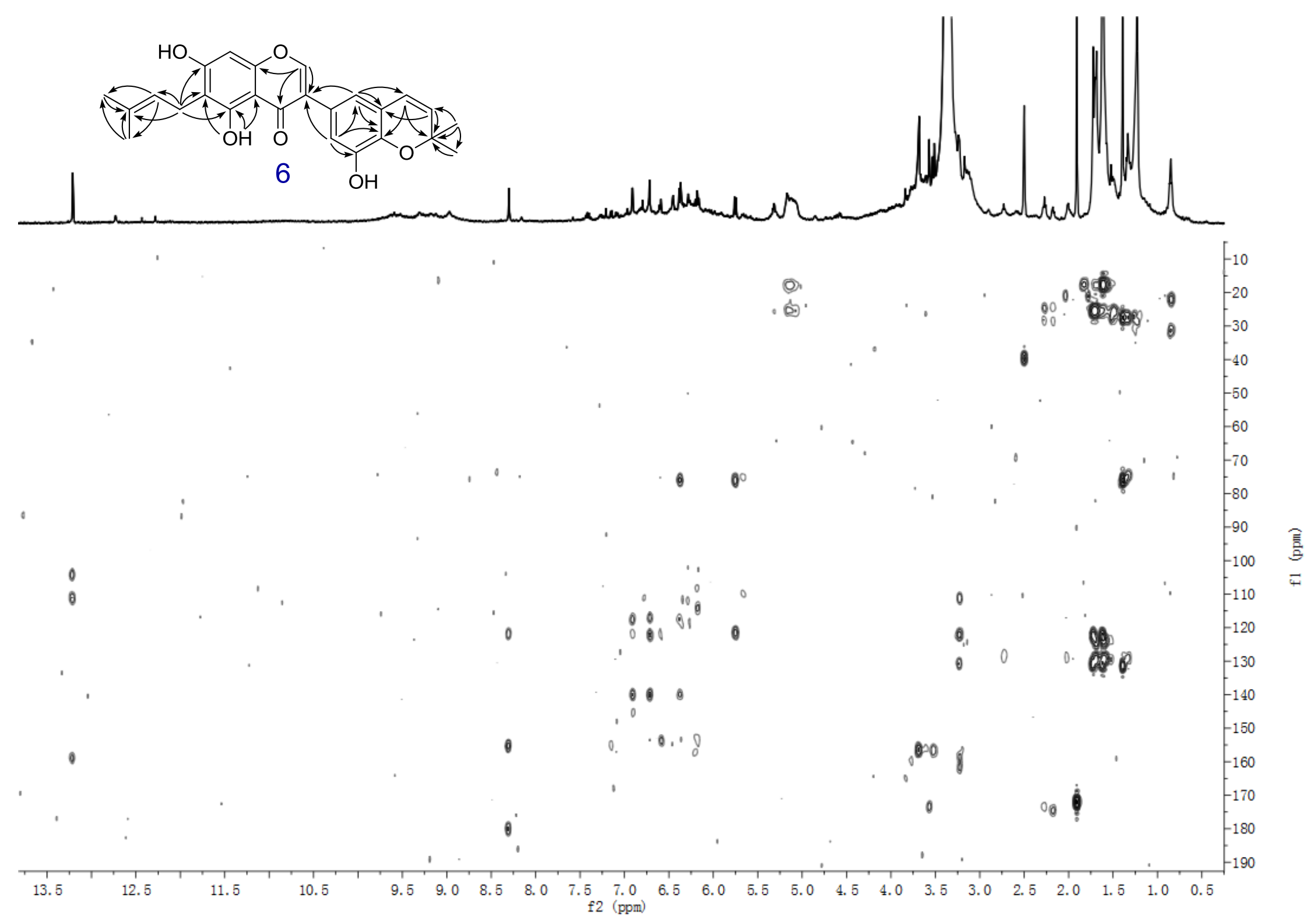

Figure S66. The HMBC spectrum of GU-MF-17 in DMSO- $d_{6}$. 

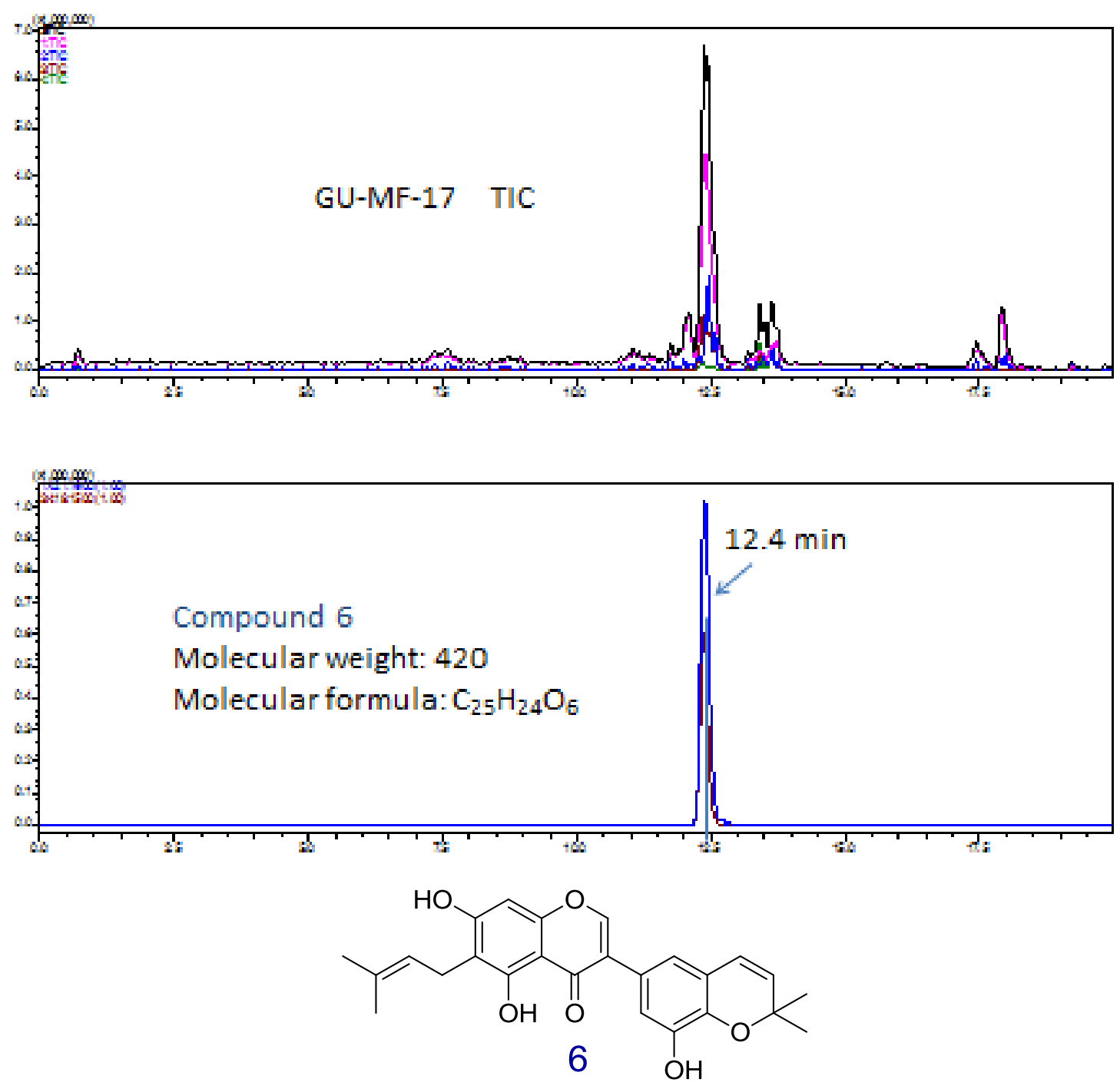

Figure S67. The IT-TOF TIC and mass chromatogram of GU-MF-17. 

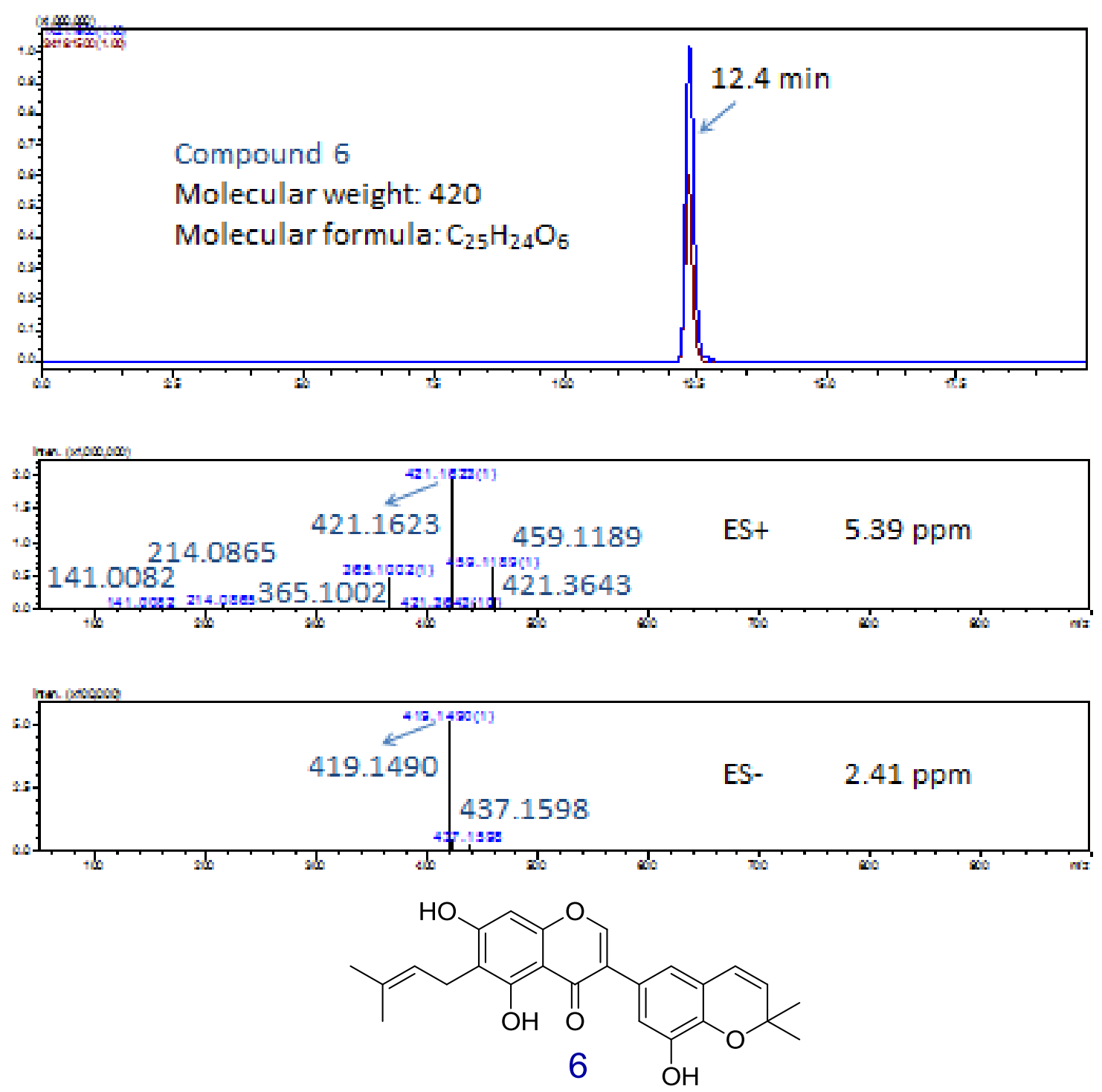

Figure S68. The (+)-HRESIMS and (-)-HRESIMS spectra of compound 6 in GU-MF-17 with extracted ions (positive and negative) for $m / z, 421$ and 419, respectively. 

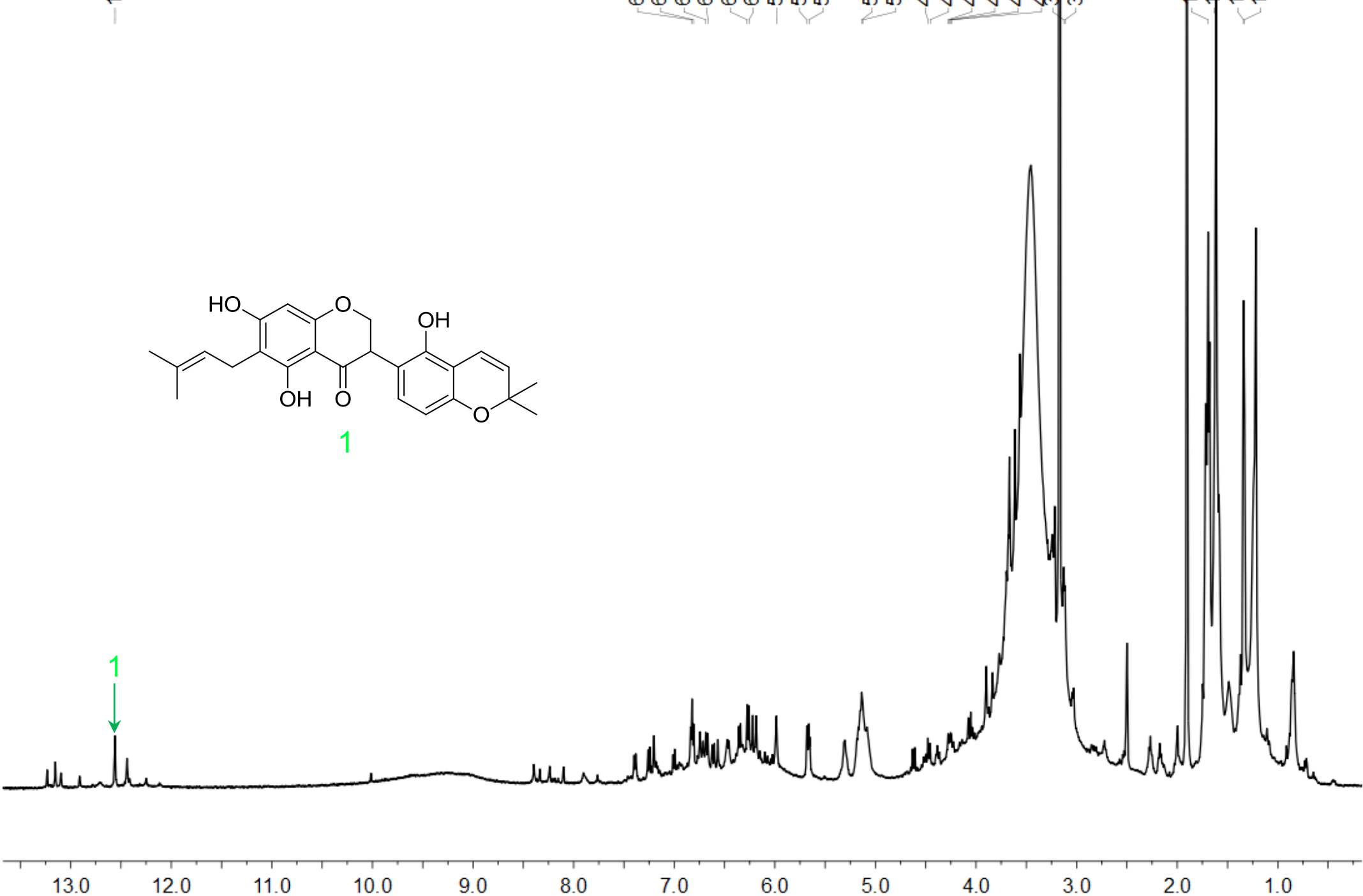

10.0

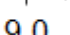

8.0
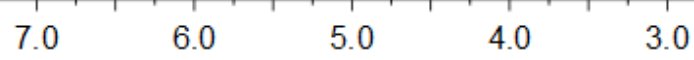

2.0

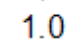

Figure S69. The ${ }^{1} \mathrm{H}$ NMR spectrum of GU-MF-18 in DMSO- $d_{6}$. 

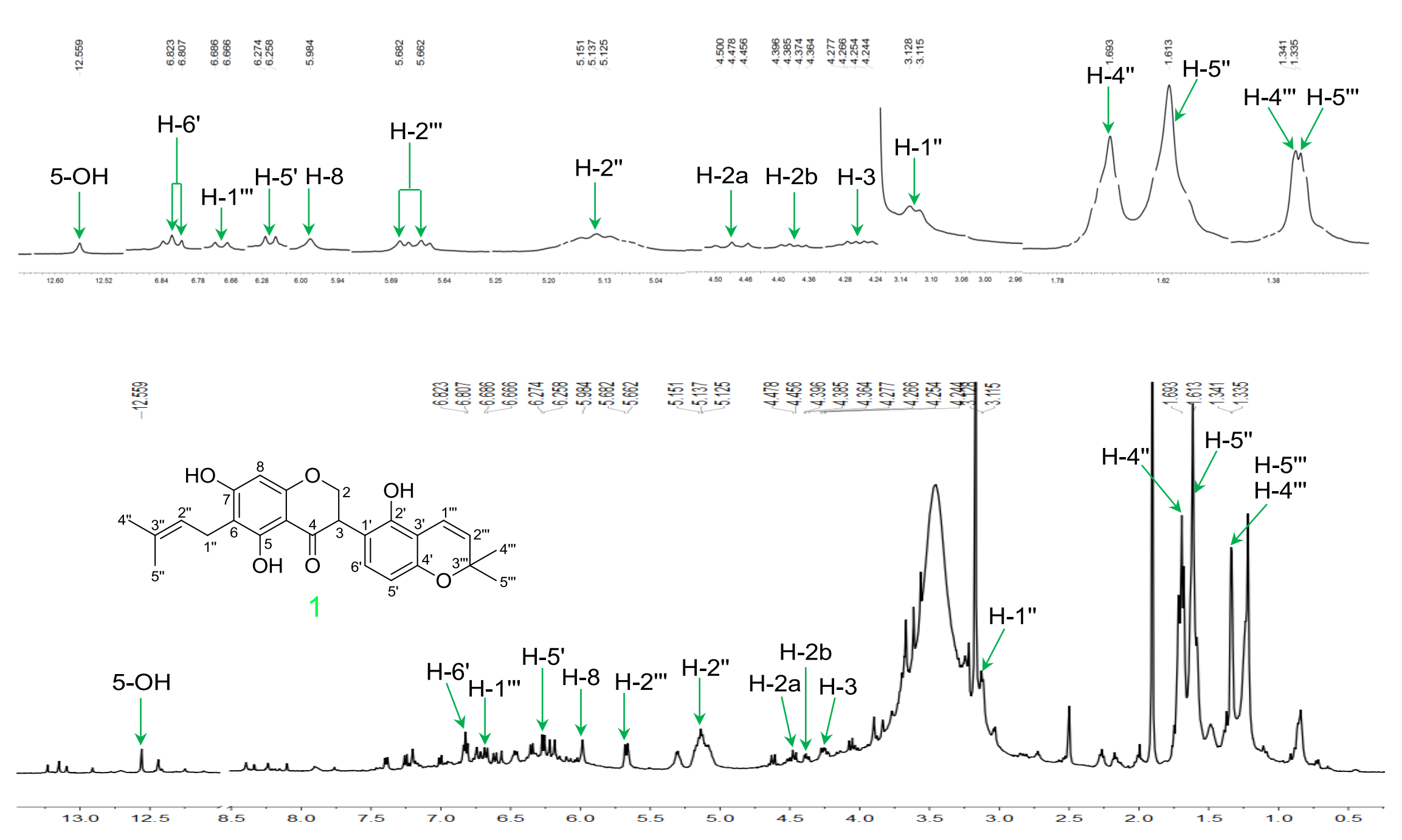

Figure S70. The ${ }^{1} \mathrm{H}$ NMR spectrum of compound $\mathbf{1}$ in GU-MF-18 in DMSO- $d_{6}$. 


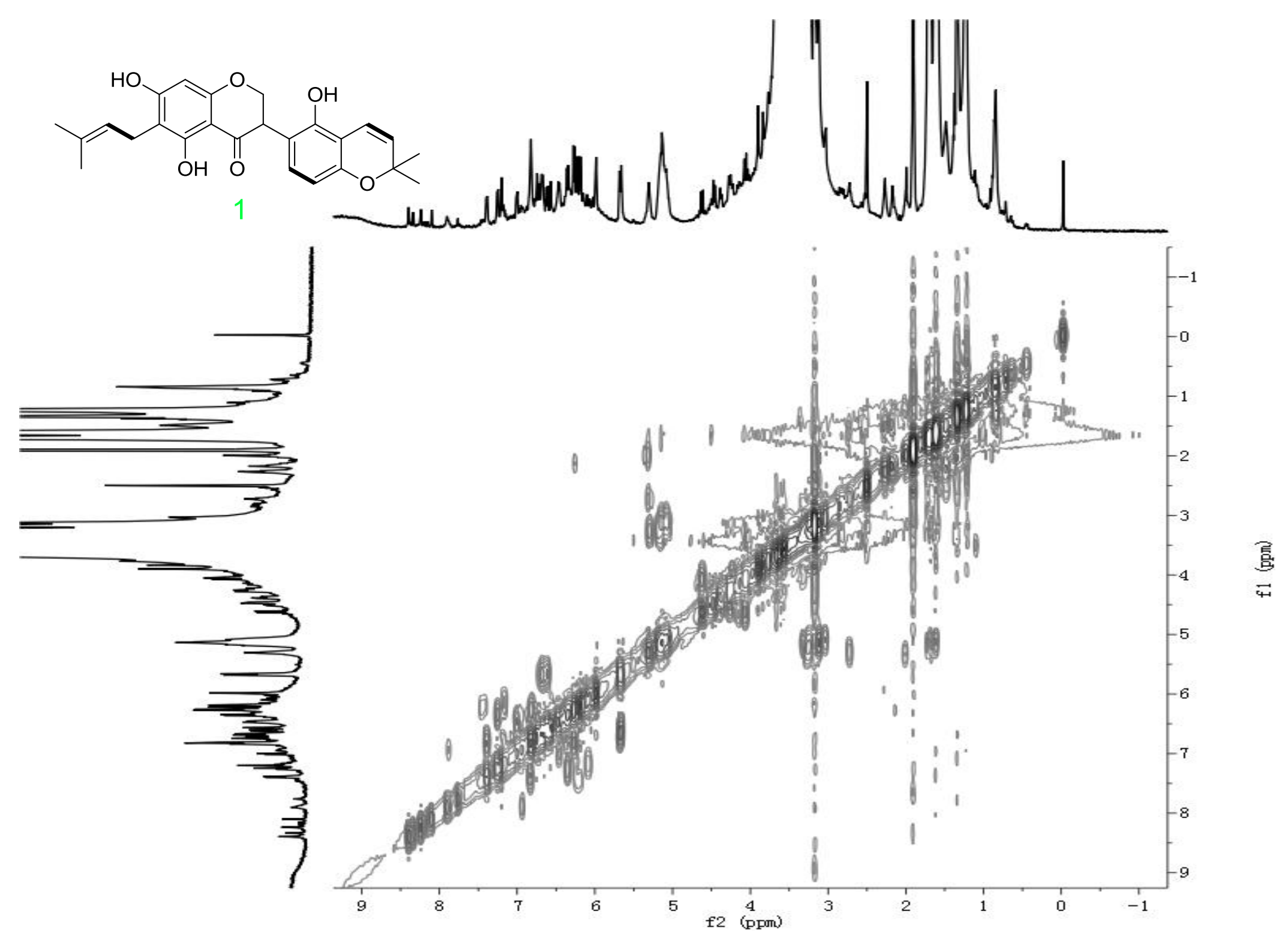

Figure S71. The ${ }^{1} \mathrm{H}^{-1} \mathrm{H}$ COSY spectrum of GU-MF-18 in DMSO- $d_{6}$. 


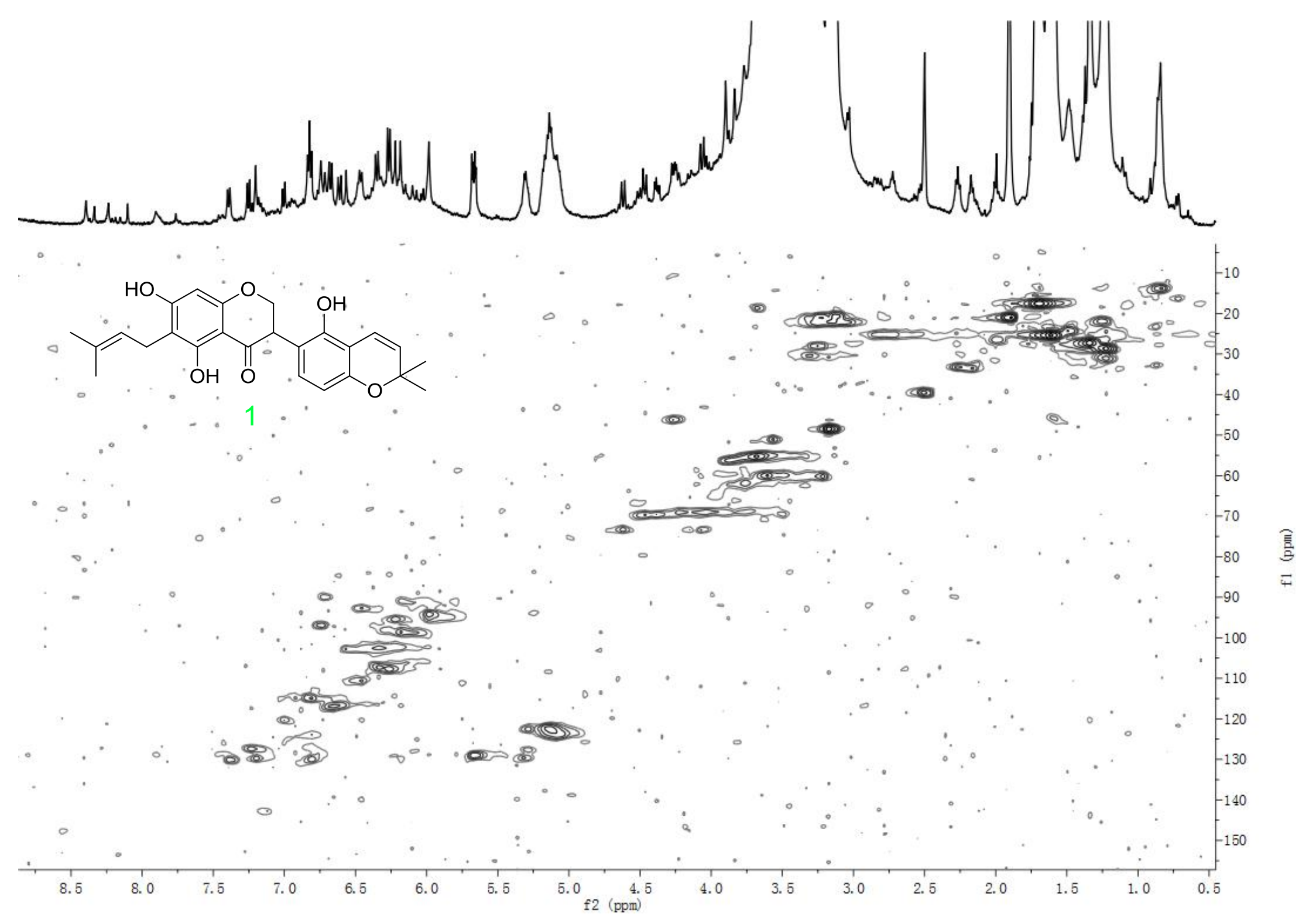

Figure S72. The HSQC spectrum of GU-MF-18 in DMSO- $d_{6}$. 


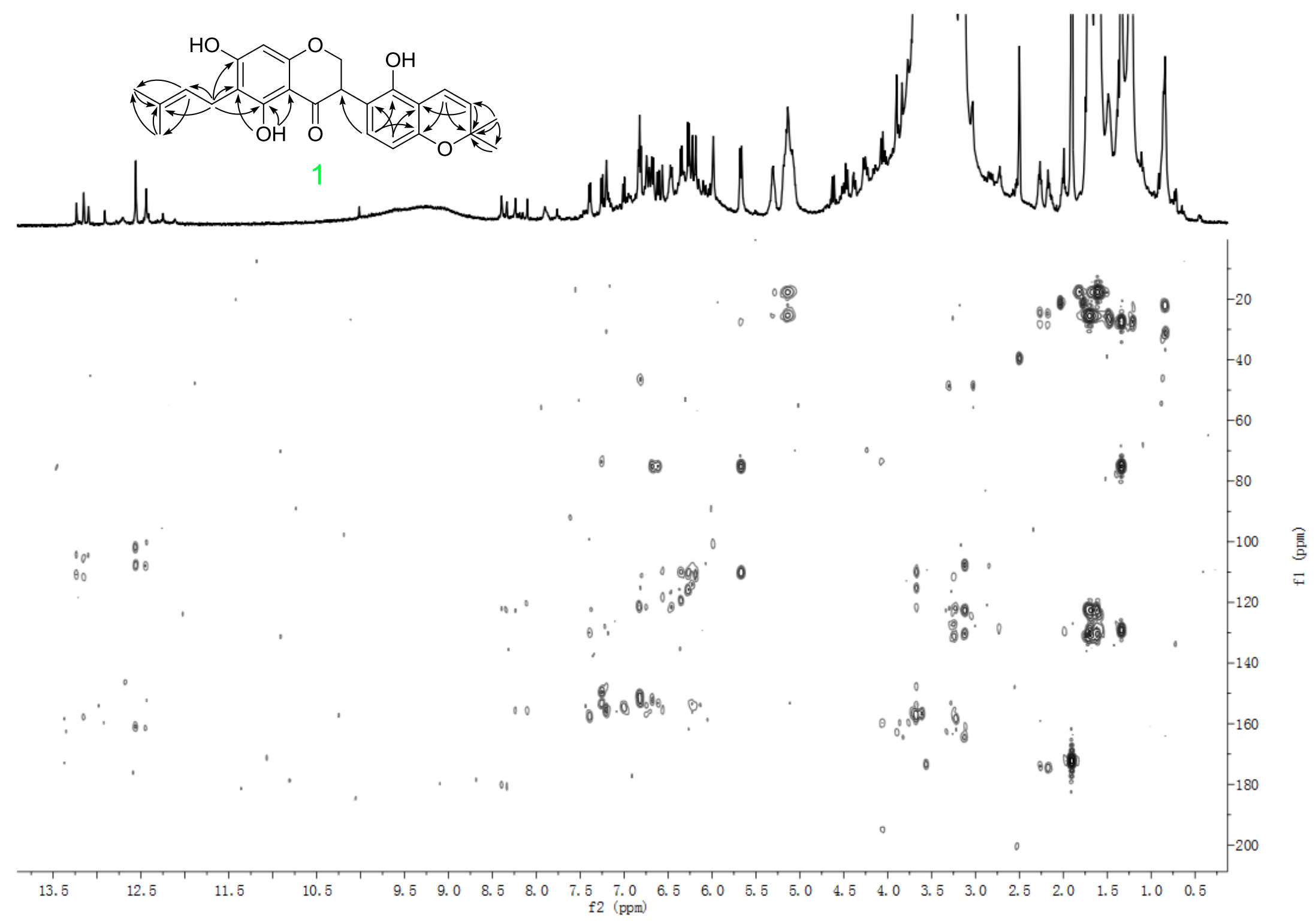

Figure S73. The HMBC spectrum of GU-MF-18 in DMSO- $d_{6}$. 

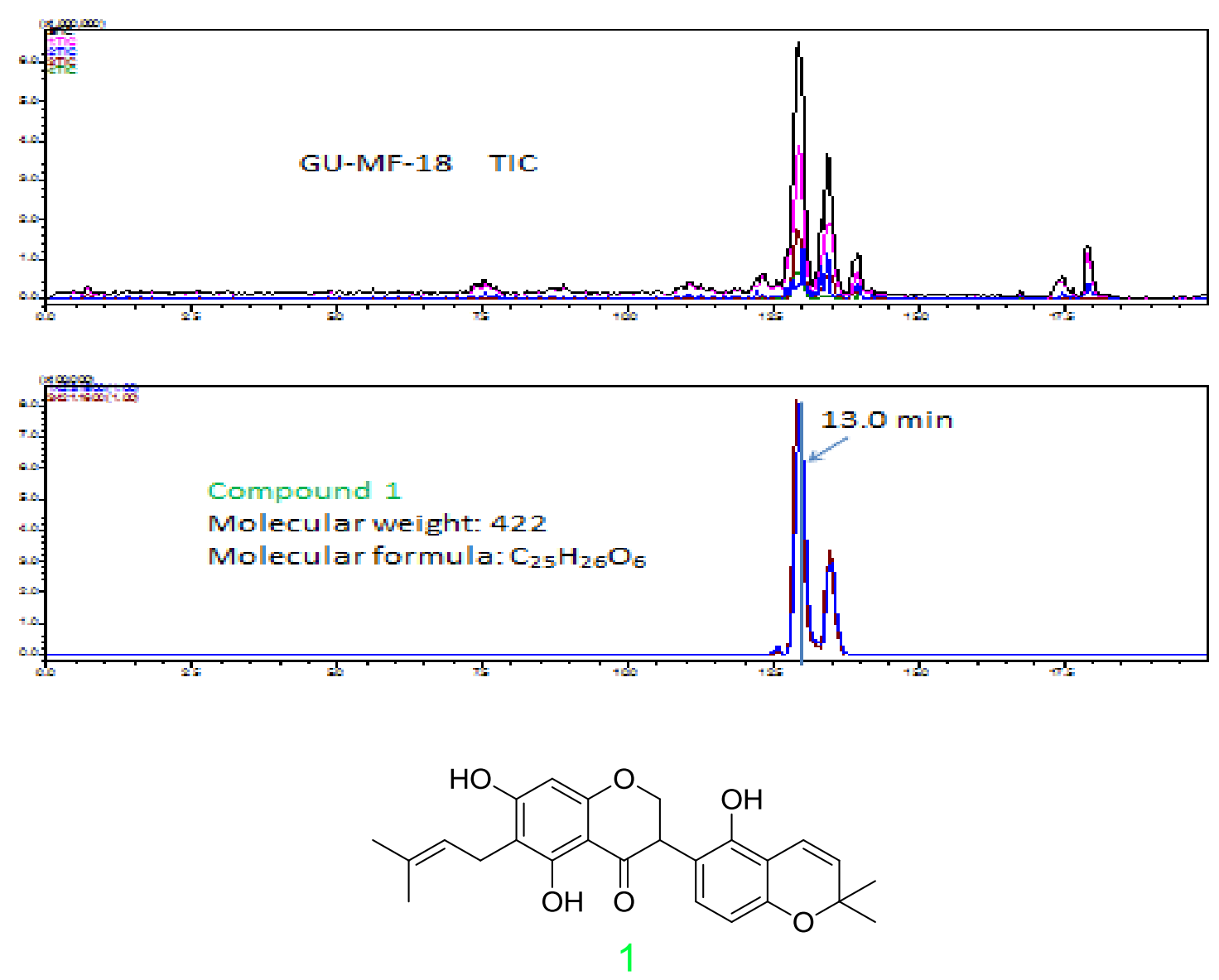

Figure S74. The IT-TOF TIC and mass chromatogram of GU-MF-18. 

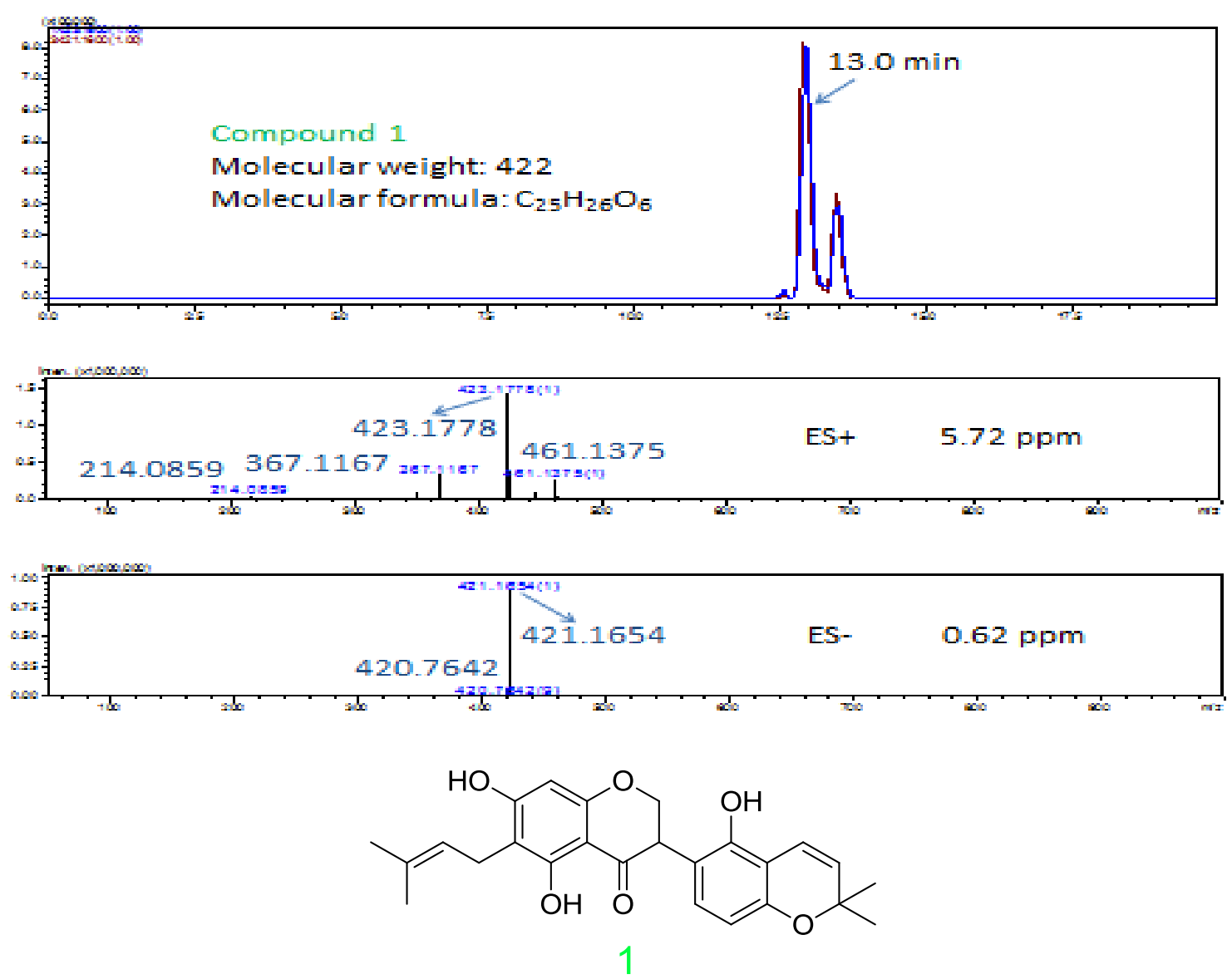

Figure S75. The (+)-HRESIMS and (-)-HRESIMS spectra of compound 1 in GU-MF-18 with extracted ions (positive and negative) for $m / z 423$ and 421 , respectively. 

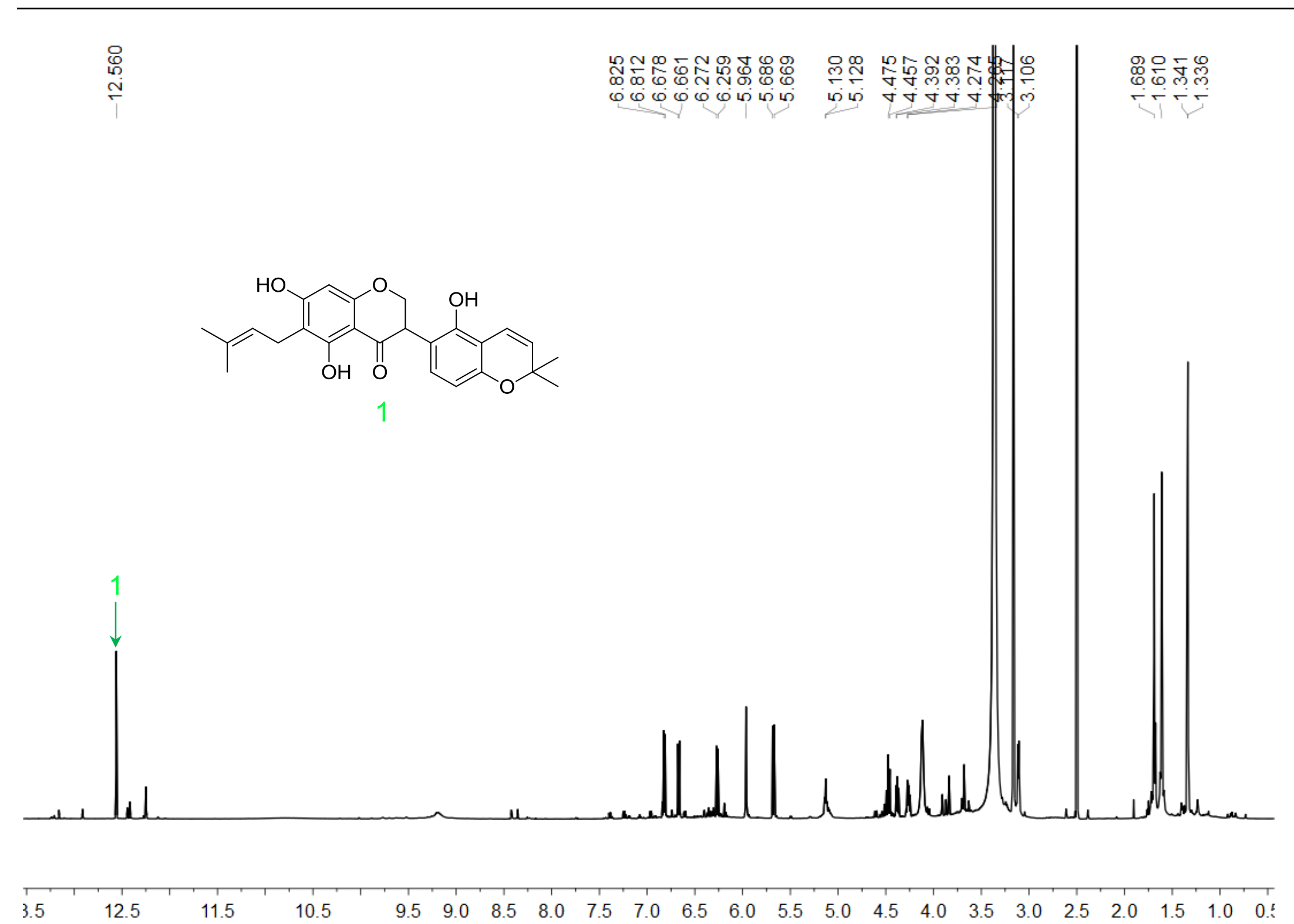

Figure S76. The ${ }^{1} \mathrm{H}$ NMR spectrum of GU-MF-18-1 in DMSO- $d_{6}$. 


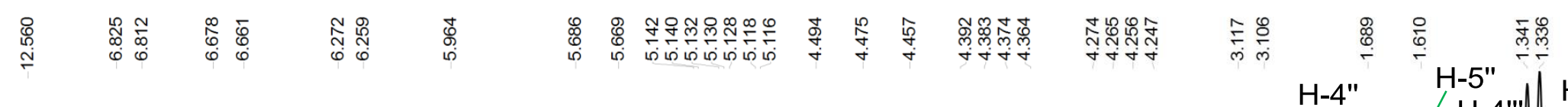

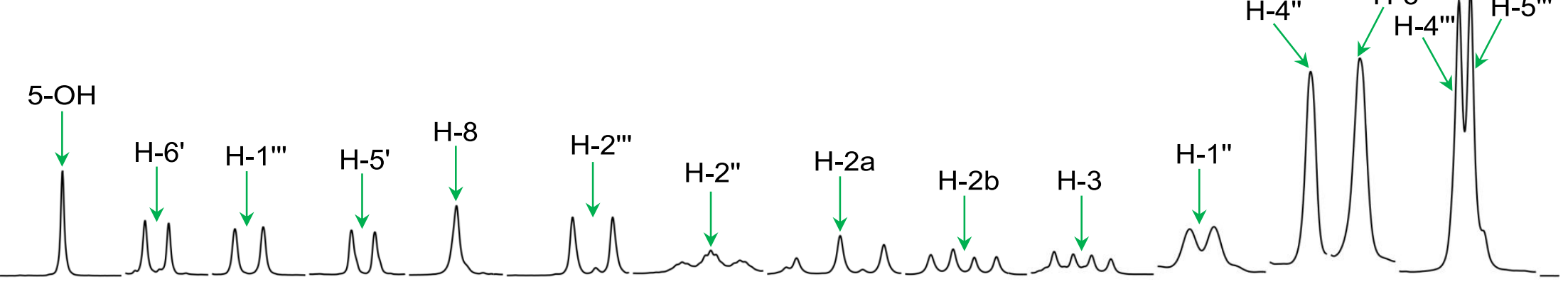

12.55 $6.80 \quad 6.66 \quad 6.27$

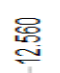
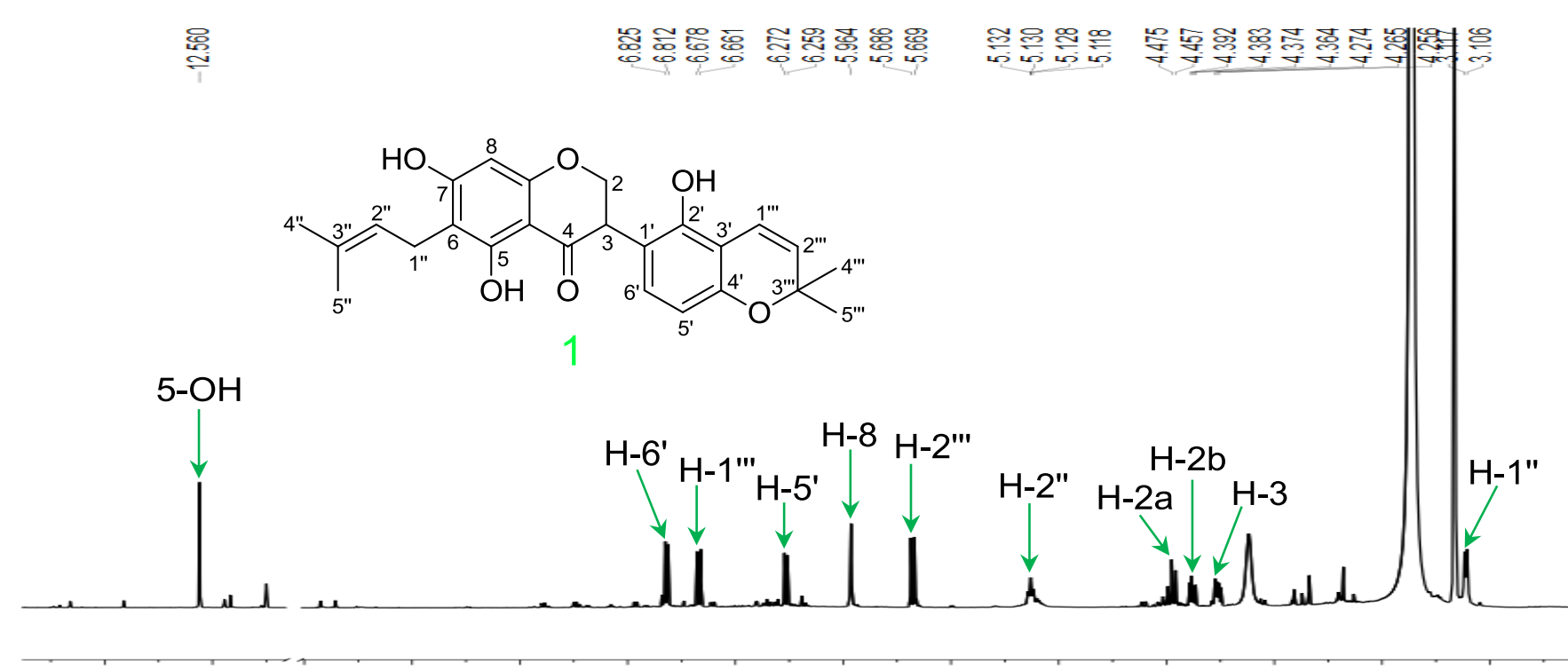

영응 훙

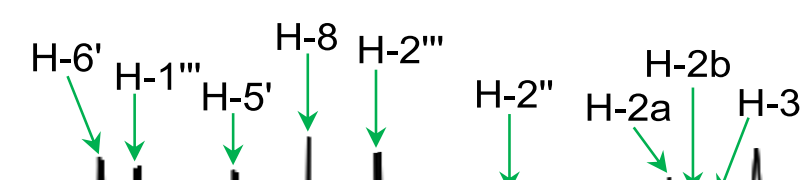

dianduld

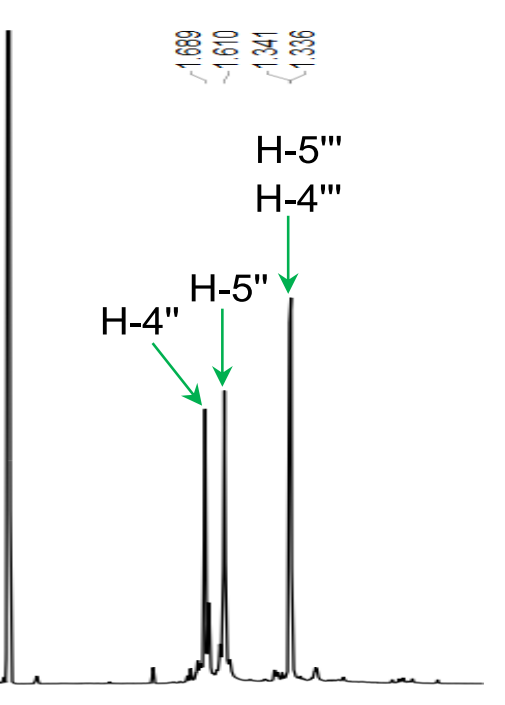

Figure S77. The ${ }^{1} \mathrm{H}$ NMR spectrum of compound 1 in GU-MF-18-1 in DMSO- $d_{6}$. 


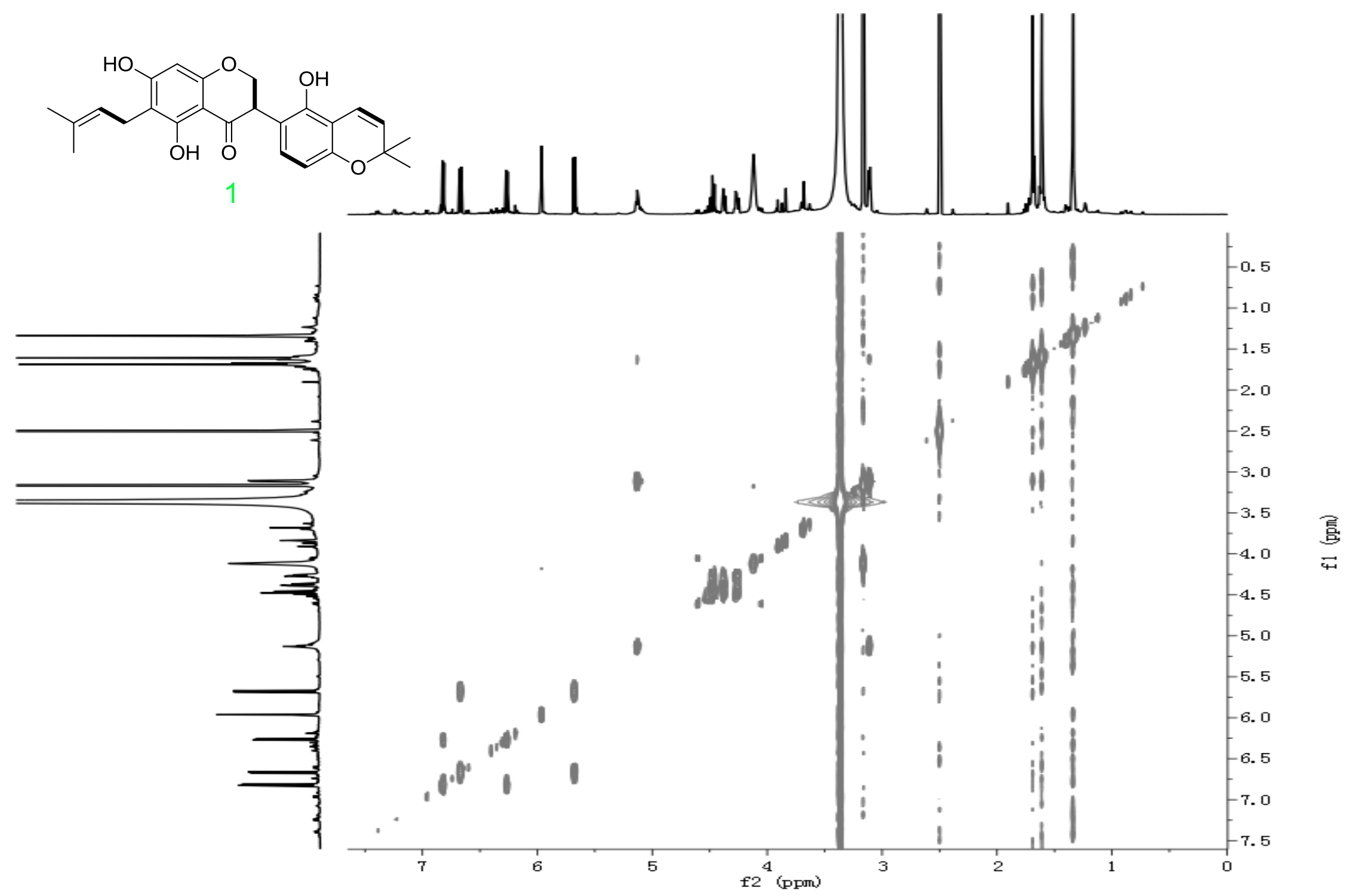

Figure S78. The ${ }^{1} \mathrm{H}-{ }^{1} \mathrm{H}$ COSY spectrum of GU-MF-18-1 in DMSO- $d_{6}$. 


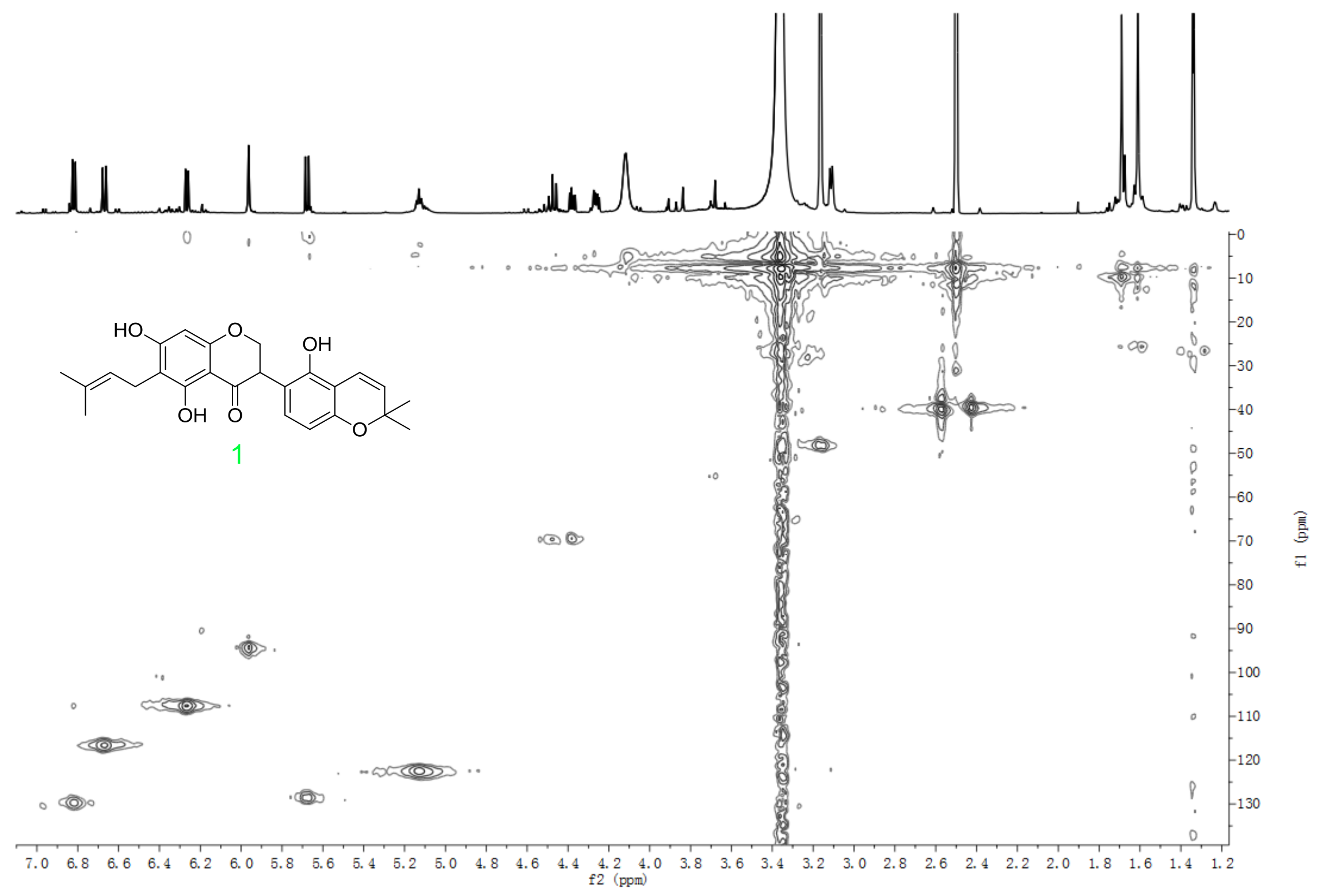

Figure S79. The HSQC spectrum of GU-MF-18-1 in DMSO- $d_{6}$. 


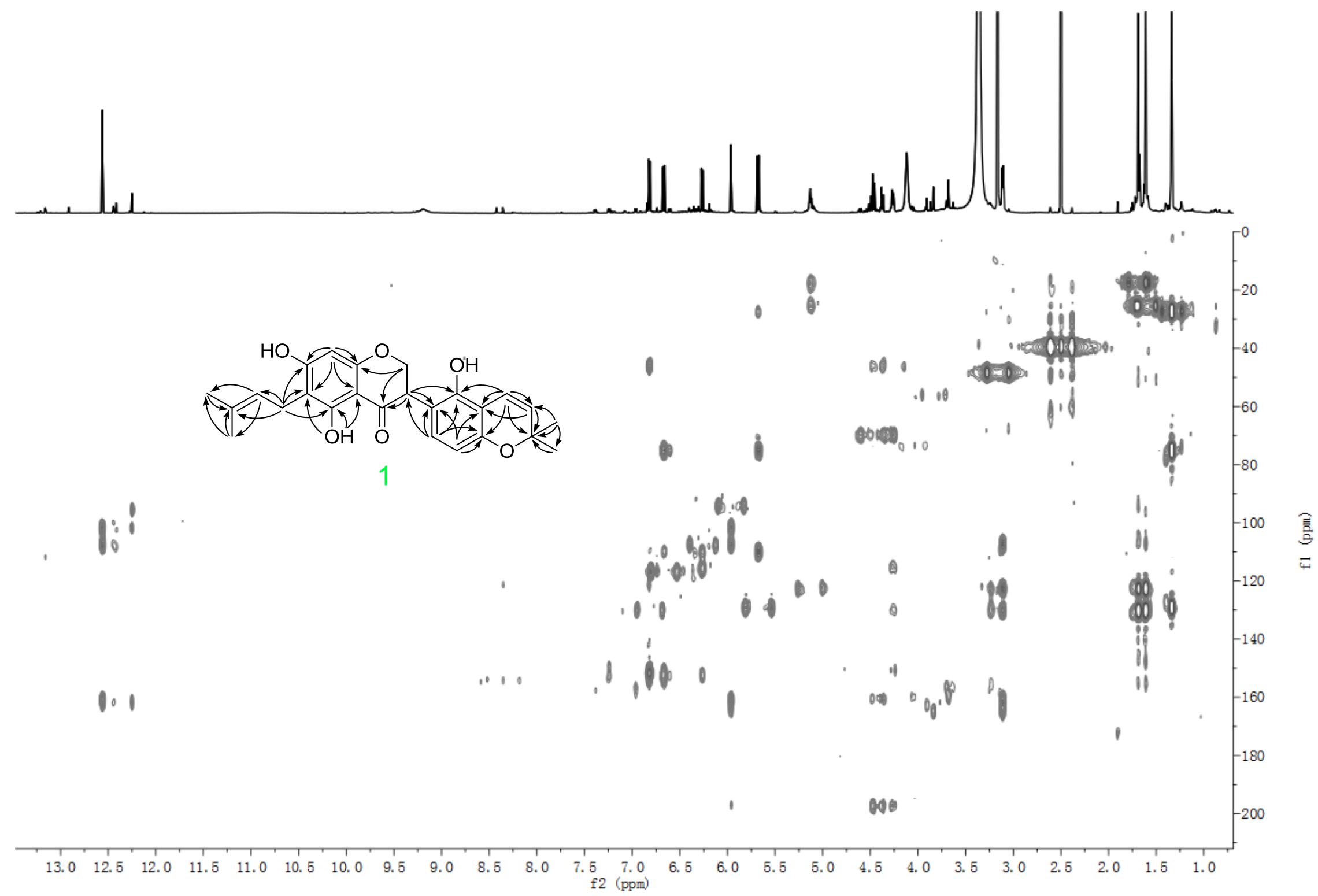

Figure S80. The HMBC spectrum of GU-MF-18-1 in DMSO- $d_{6}$. 


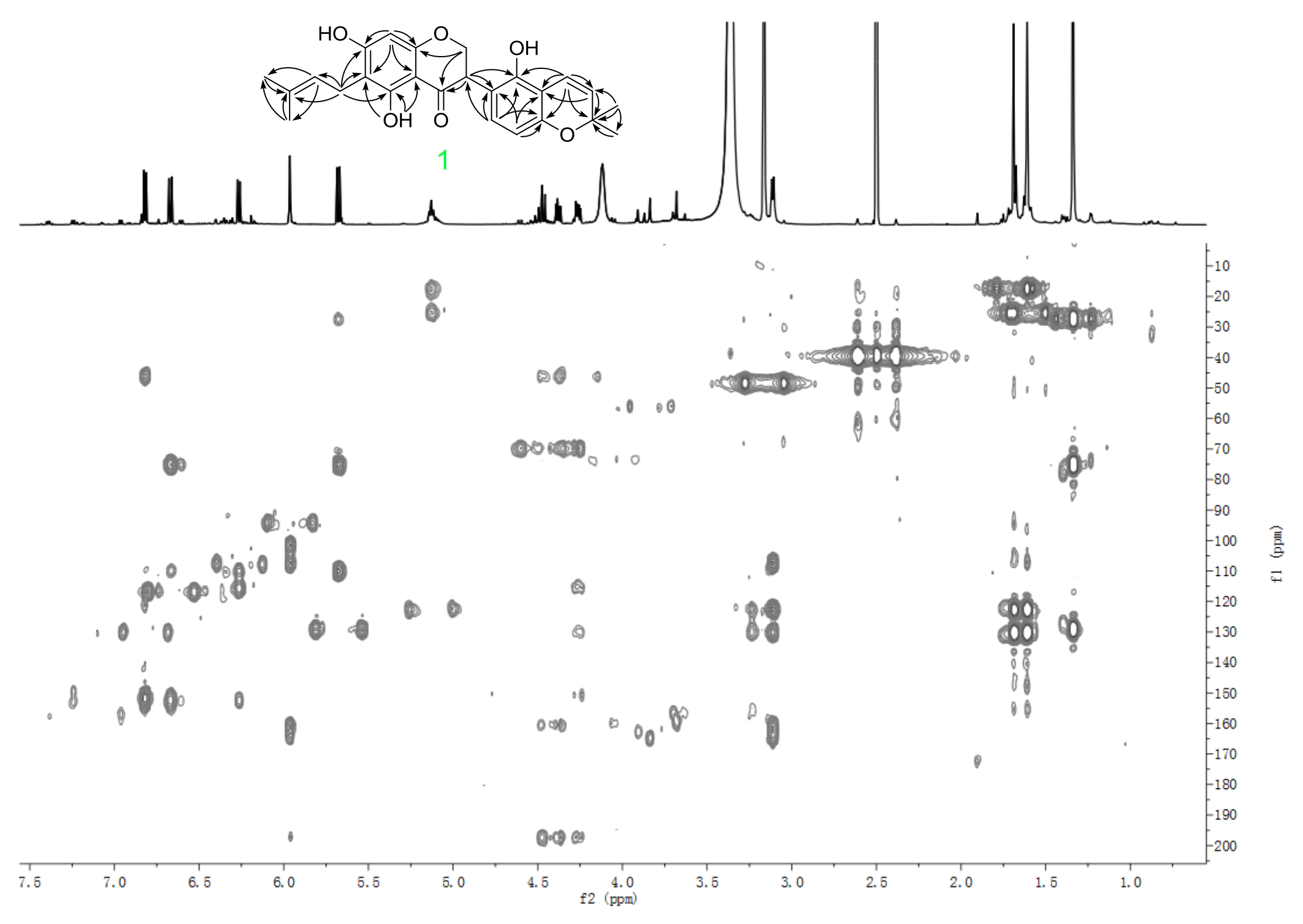

Figure S81. Expansion of the HMBC spectrum of GU-MF-18-1 for the range $\delta_{\mathrm{H}} 0.55-7.56$ and $\delta_{\mathrm{C}} 2.5-206.0$. 

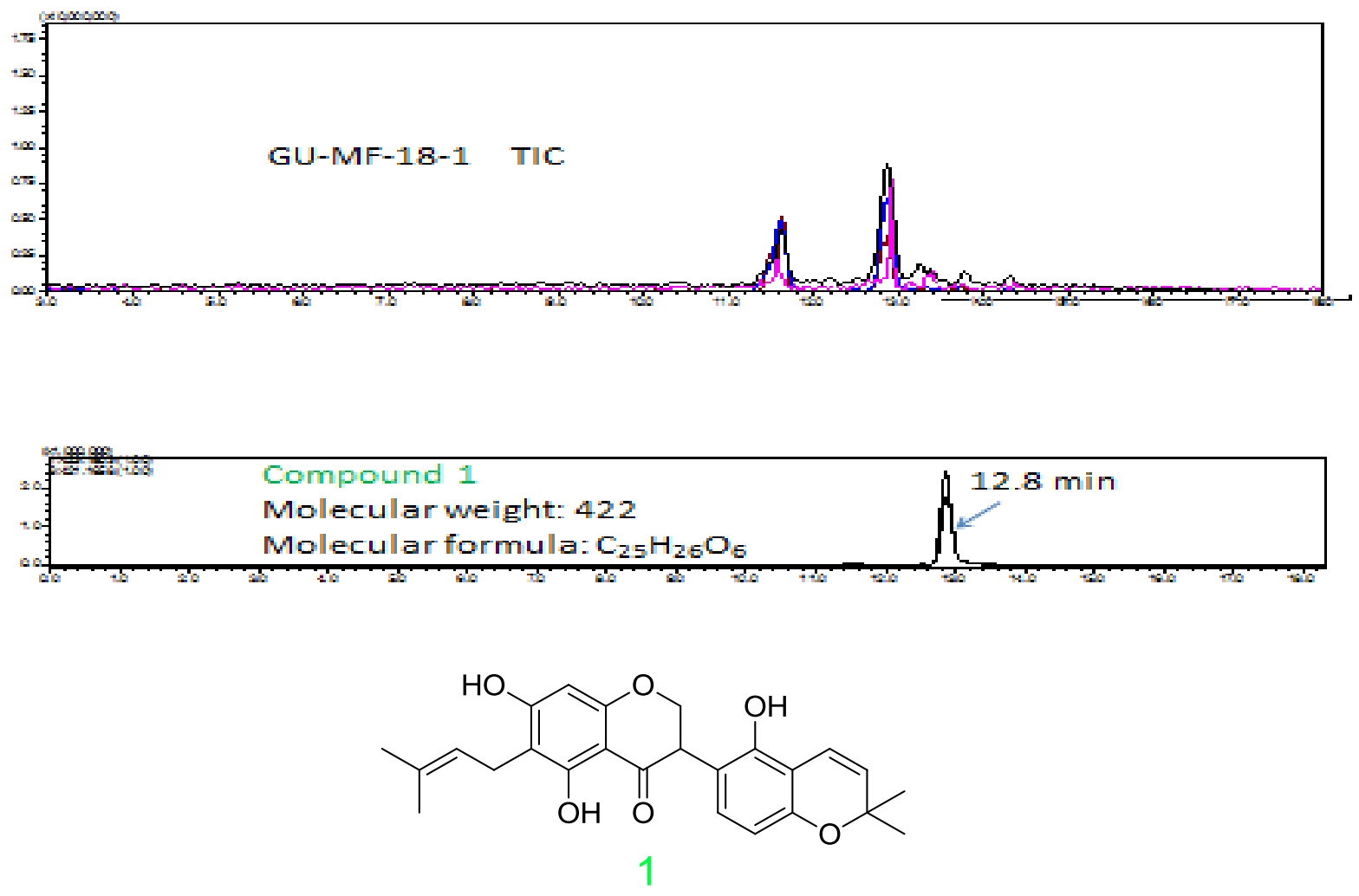

Figure S82. The IT-TOF TIC and mass chromatogram of GU-MF-18-1. 

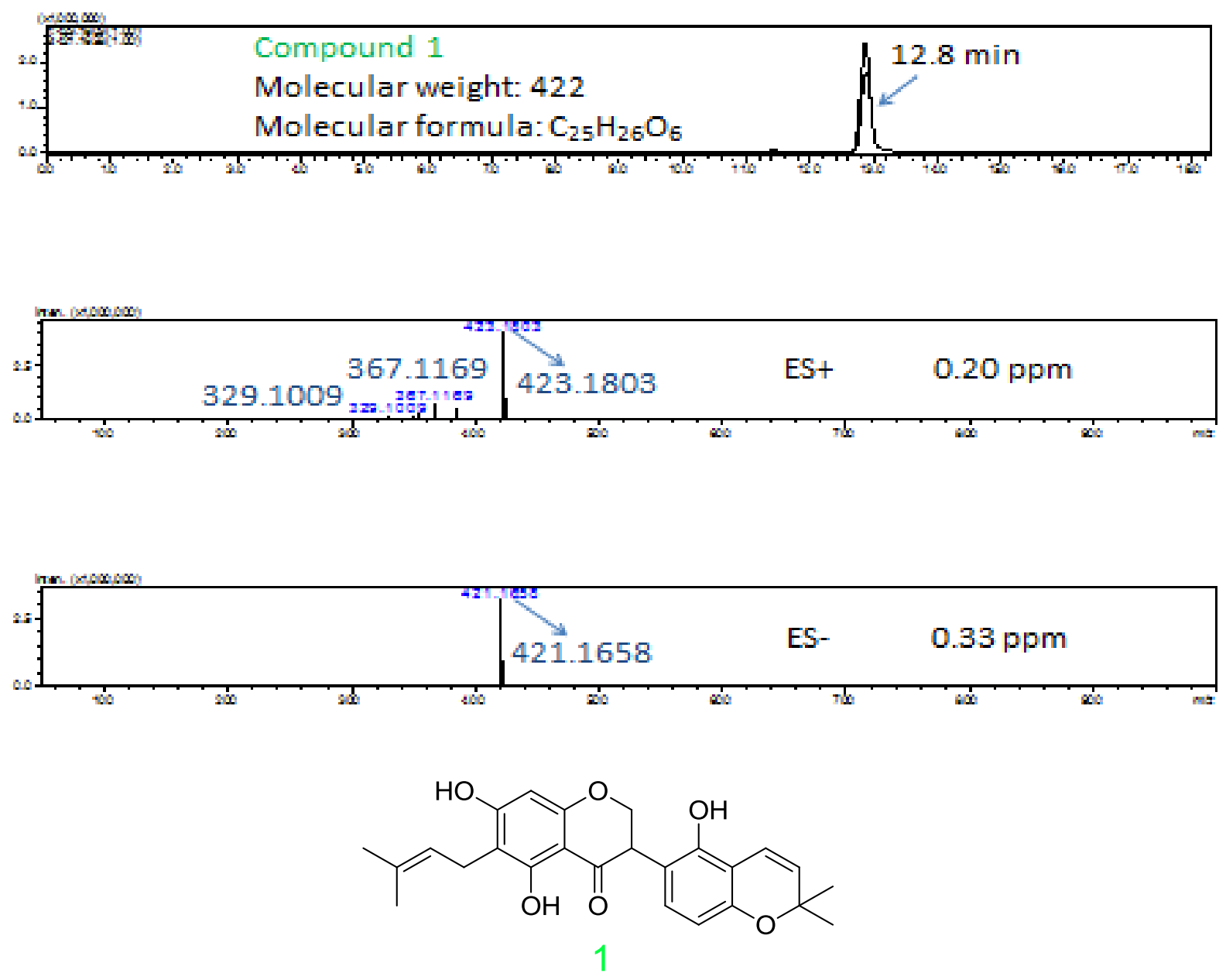

Figure S83. The (+)-HRESIMS and (-)-HRESIMS spectra of compound 1 in GU-MF-18-1 with extracted ions (positive and negative) for $m / z, 423$ and 421, respectively. 

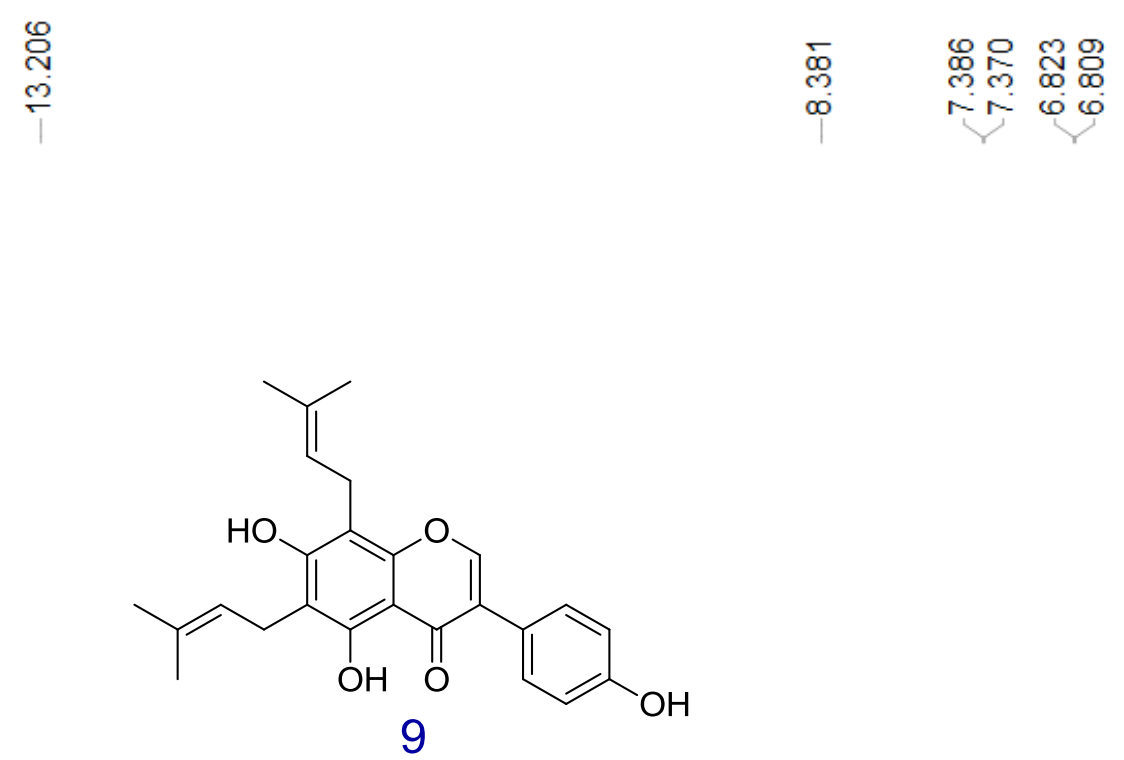

$\frac{\text { 군 }}{5}$
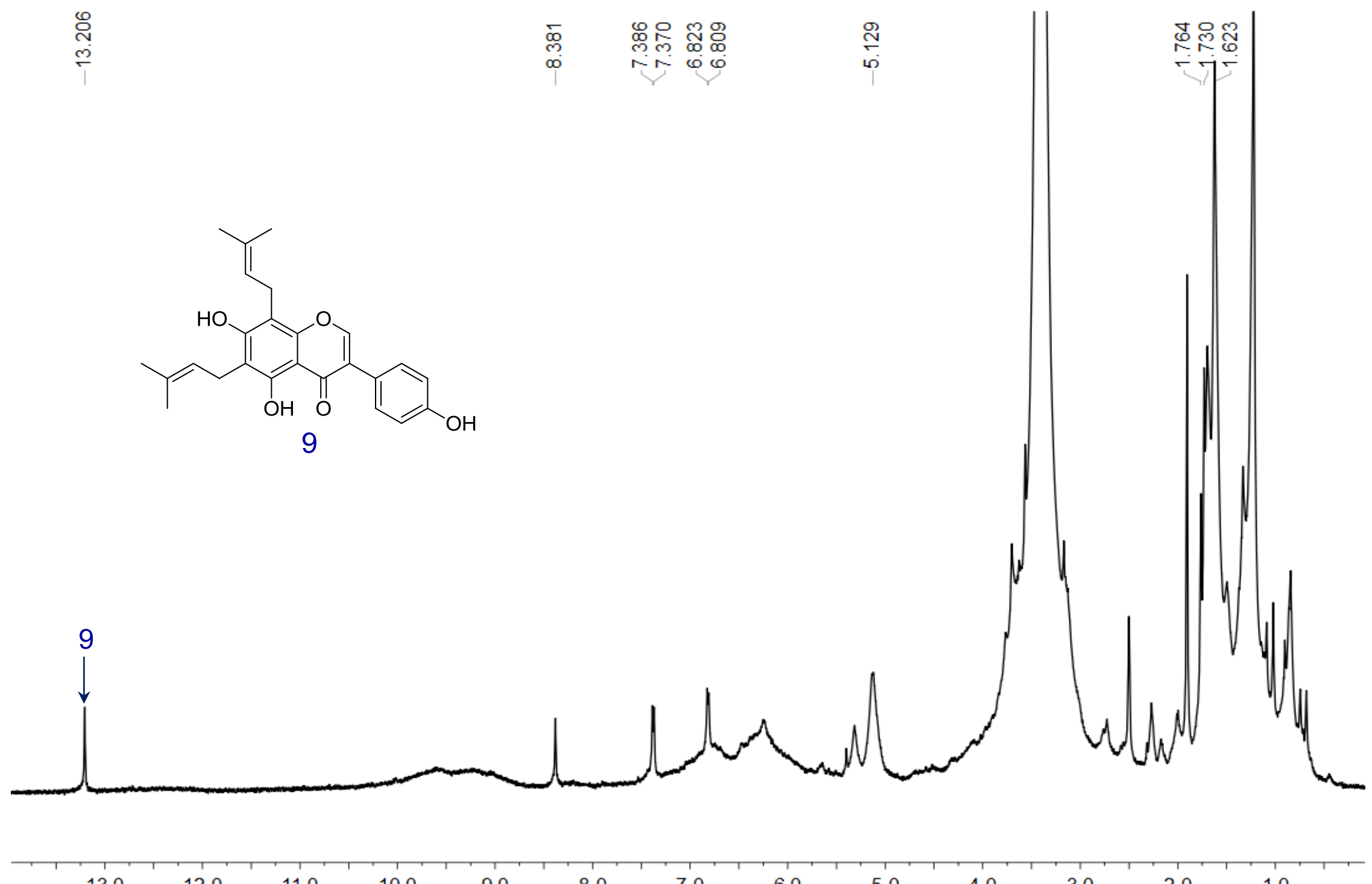

13.0
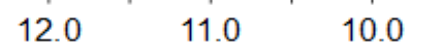

9.0

8.0

7.0

6.0

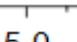

4.0

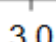

20

1.0

Figure S84. The ${ }^{1} \mathrm{H}$ NMR spectrum of GU-MF-19 in DMSO- $d_{6}$. 


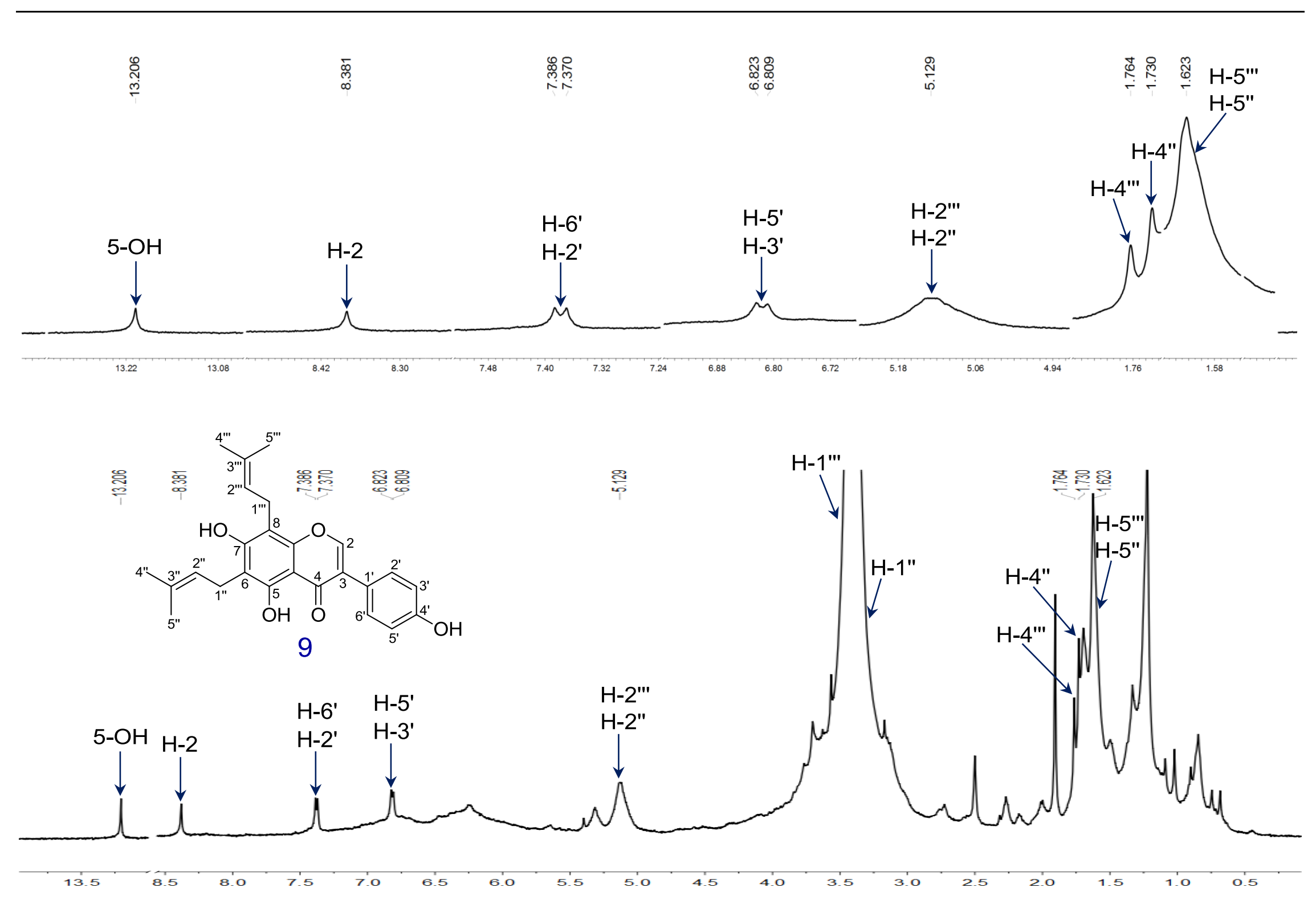

Figure S85. The ${ }^{1} \mathrm{H}$ NMR spectrum of compound 9 in GU-MF-19 in DMSO- $d_{6}$. 


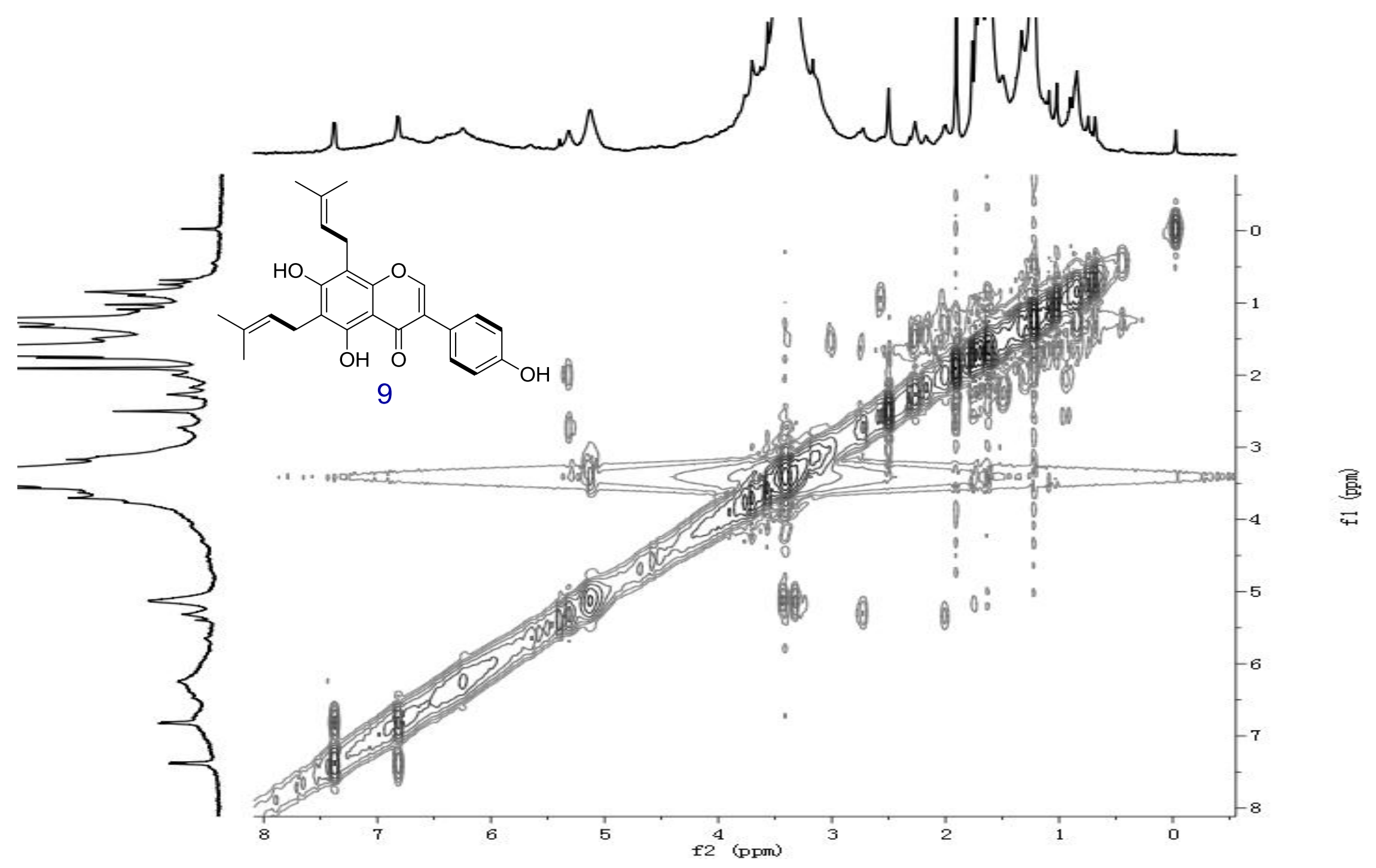

Figure S86. The ${ }^{1} \mathrm{H}-{ }^{1} \mathrm{H}$ COSY spectrum of GU-MF-19 in DMSO- $d_{6}$. 


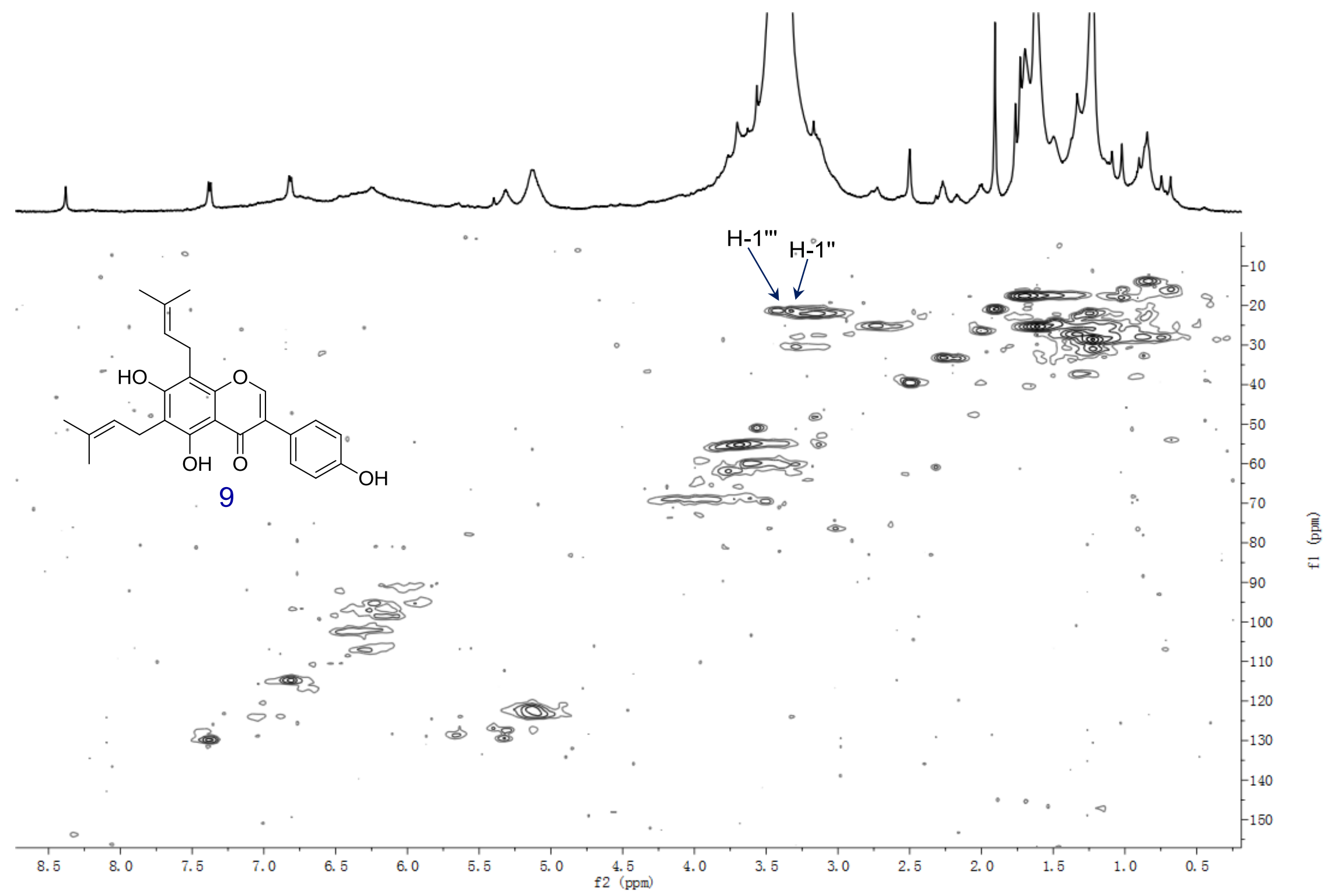

Figure S87. The HSQC spectrum of GU-MF-19 in DMSO- $d_{6}$. 


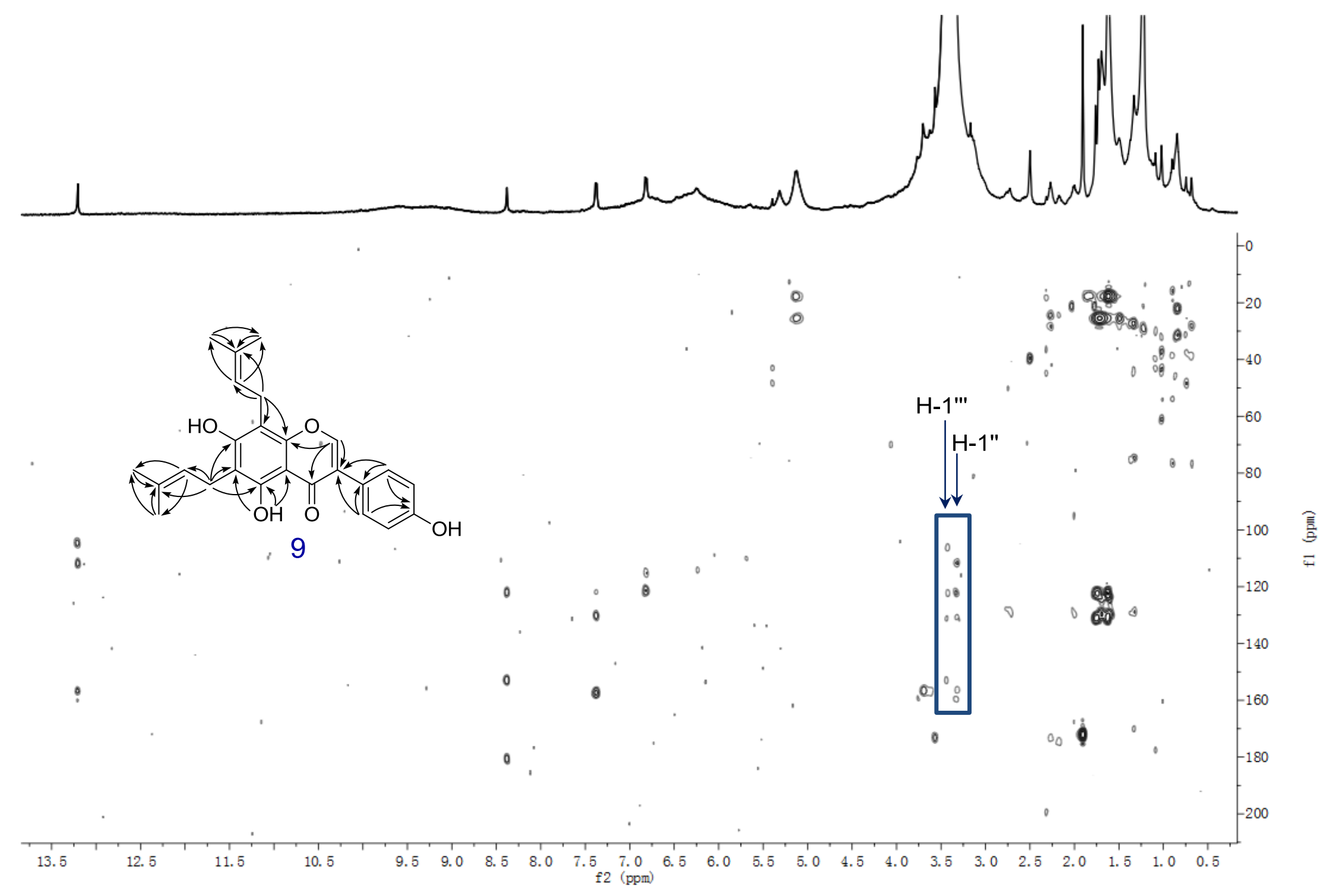

Figure S88. The HMBC spectrum of GU-MF-19 in DMSO- $d_{6}$. 

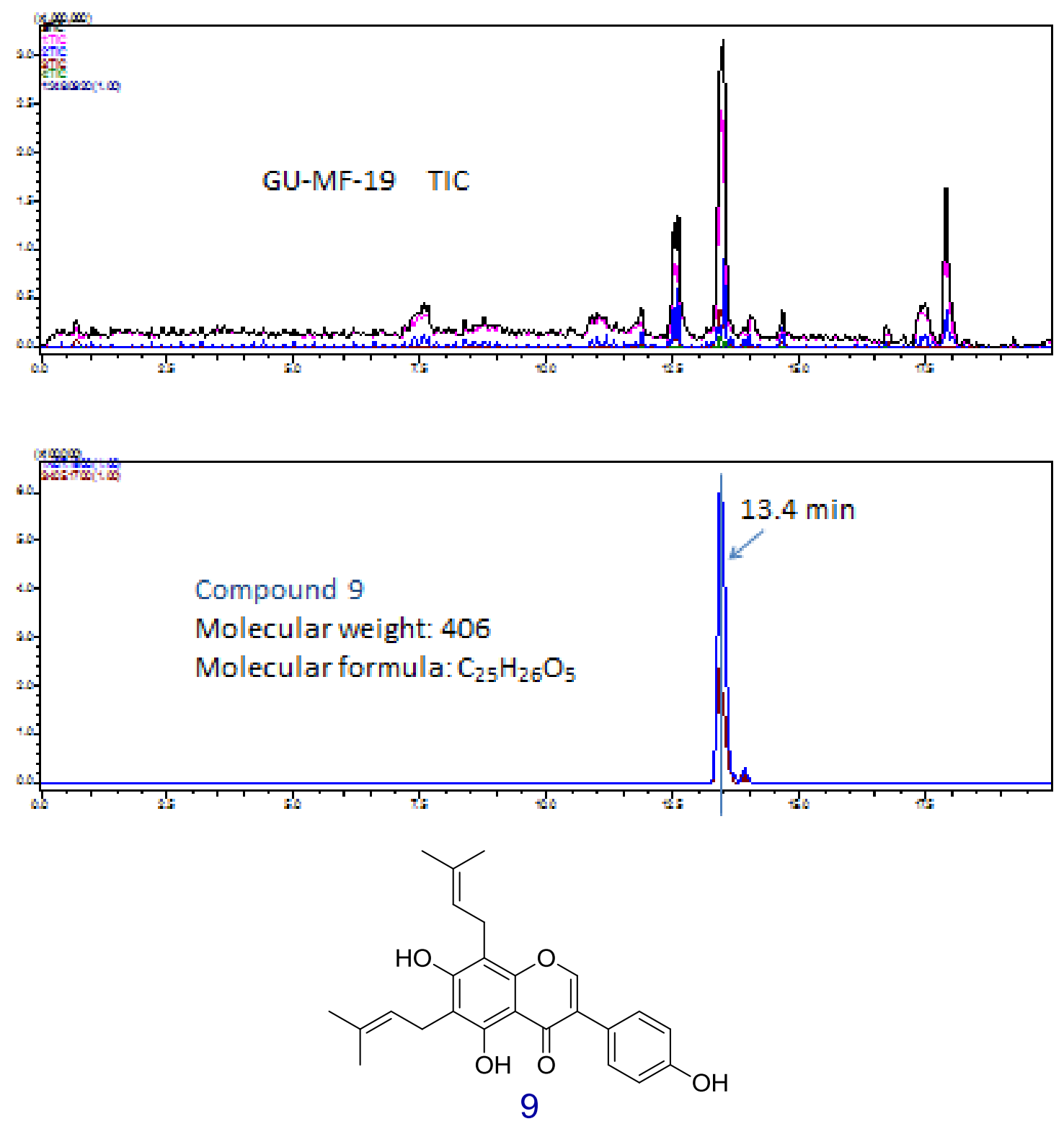

Figure S89. The IT-TOF TIC and mass chromatogram of GU-MF-19. 

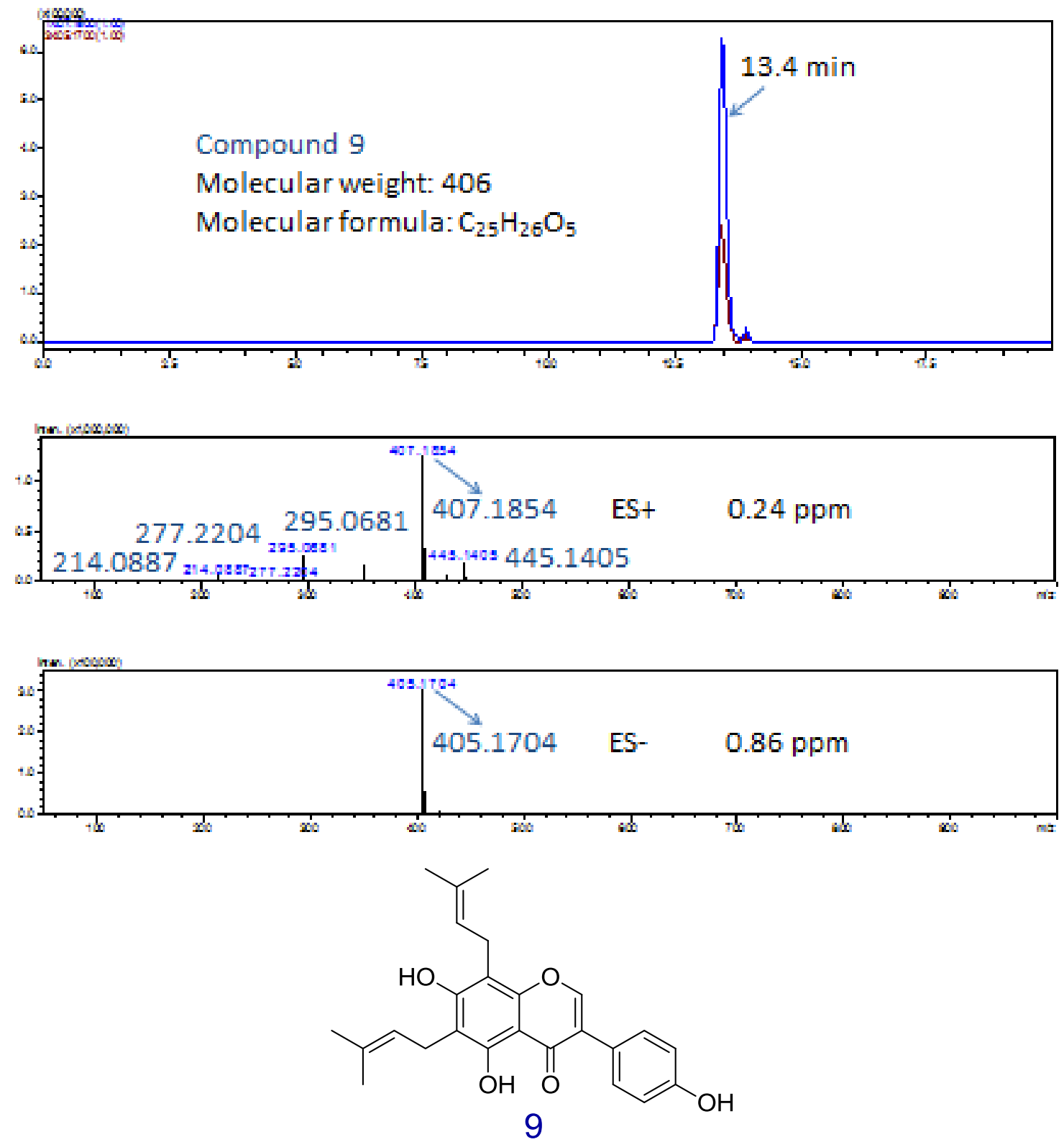

Figure S90. The (+)-HRESIMS and (-)-HRESIMS spectra of compound 9 in GU-MF-19 with extracted ions (positive and negative) for $\mathrm{m} / \mathrm{z}, 407$ and 405, respectively. 

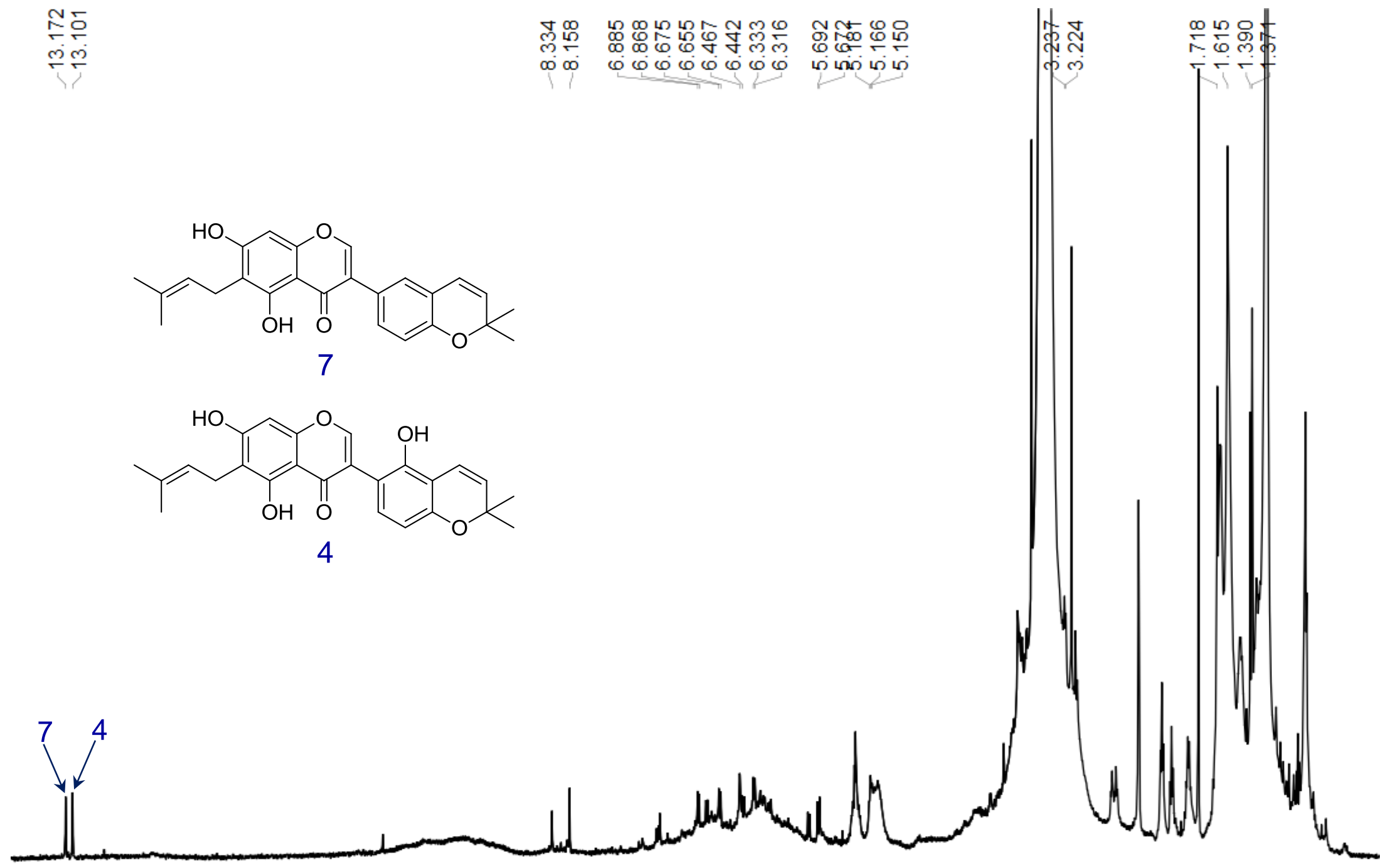

13.0

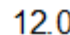

11.0

9.0

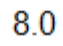

7.0

6.0

5.0

Figure S91. The ${ }^{1} \mathrm{H}$ NMR spectrum of GU-MF-20 in DMSO- $d_{6}$. 


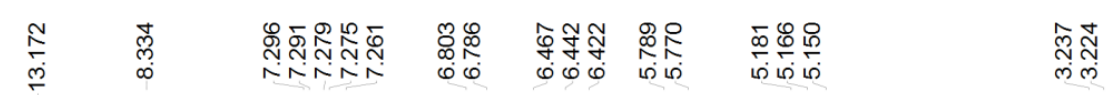

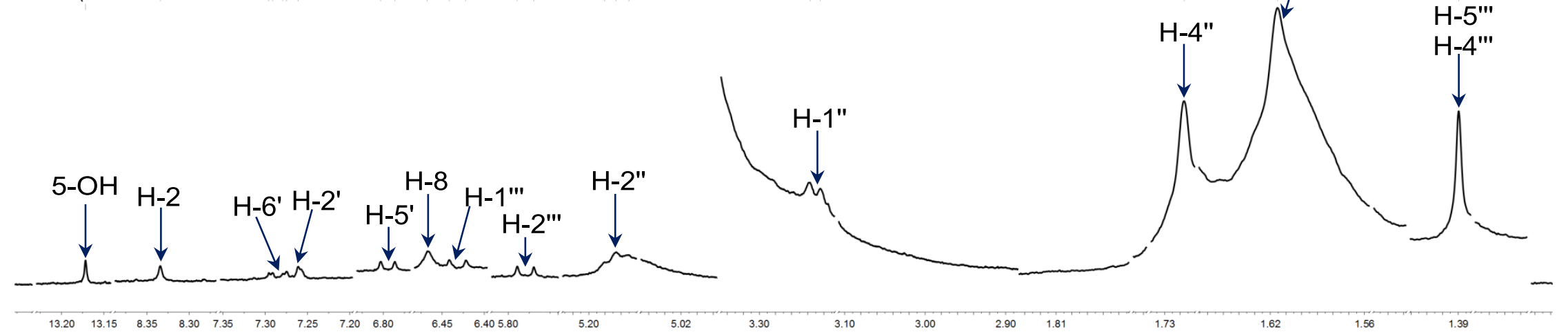

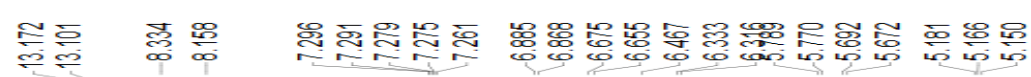
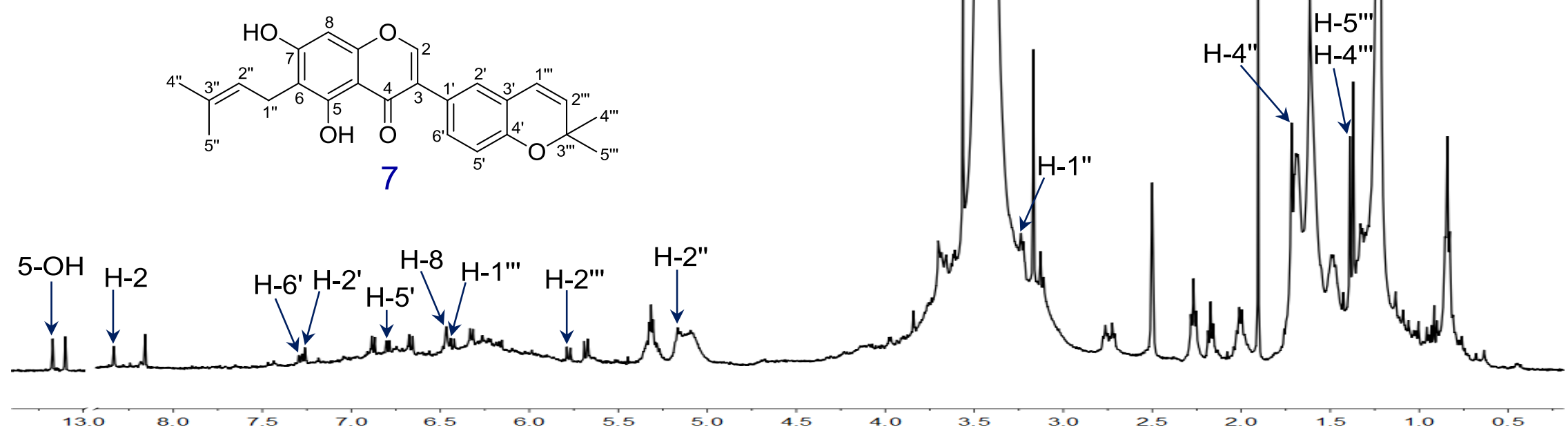

Figure S92. The ${ }^{1} \mathrm{H}$ NMR spectrum of compound 7 in GU-MF-20 in DMSO- $d_{6}$. 

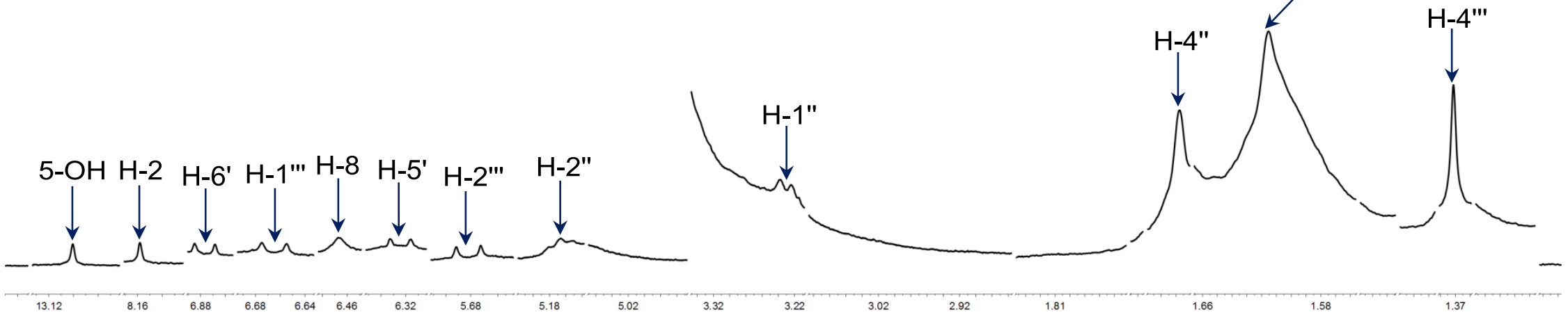
$\begin{array}{lllll}68 & 6.64 & 6.46 & 6.32 & 5.68\end{array}$
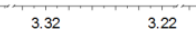

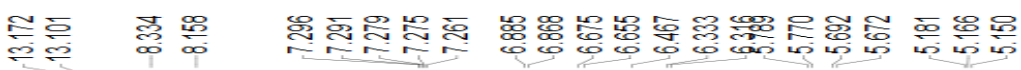
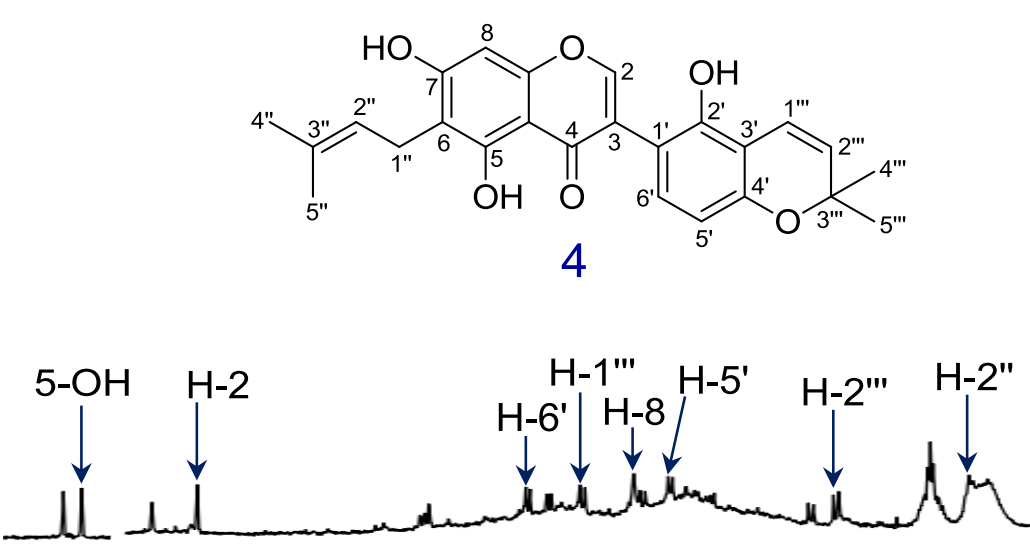

Figure S93. The ${ }^{1} \mathrm{H}$ NMR spectrum of compound 4 in GU-MF-20 in DMSO- $d_{6}$. 


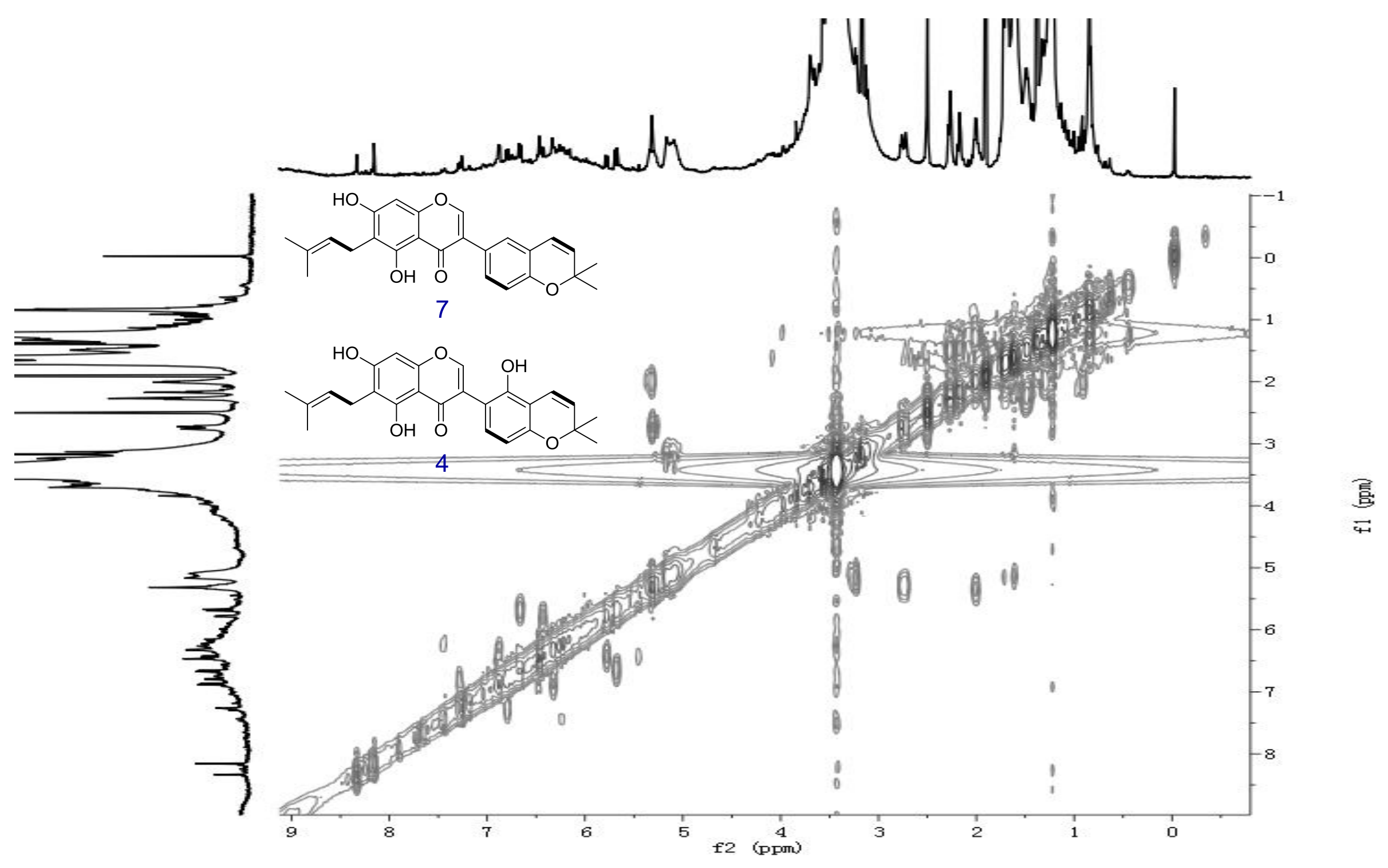

Figure S94. The ${ }^{1} \mathrm{H}^{-1} \mathrm{H}$ COSY spectrum of GU-MF-20 in DMSO- $d_{6}$. 


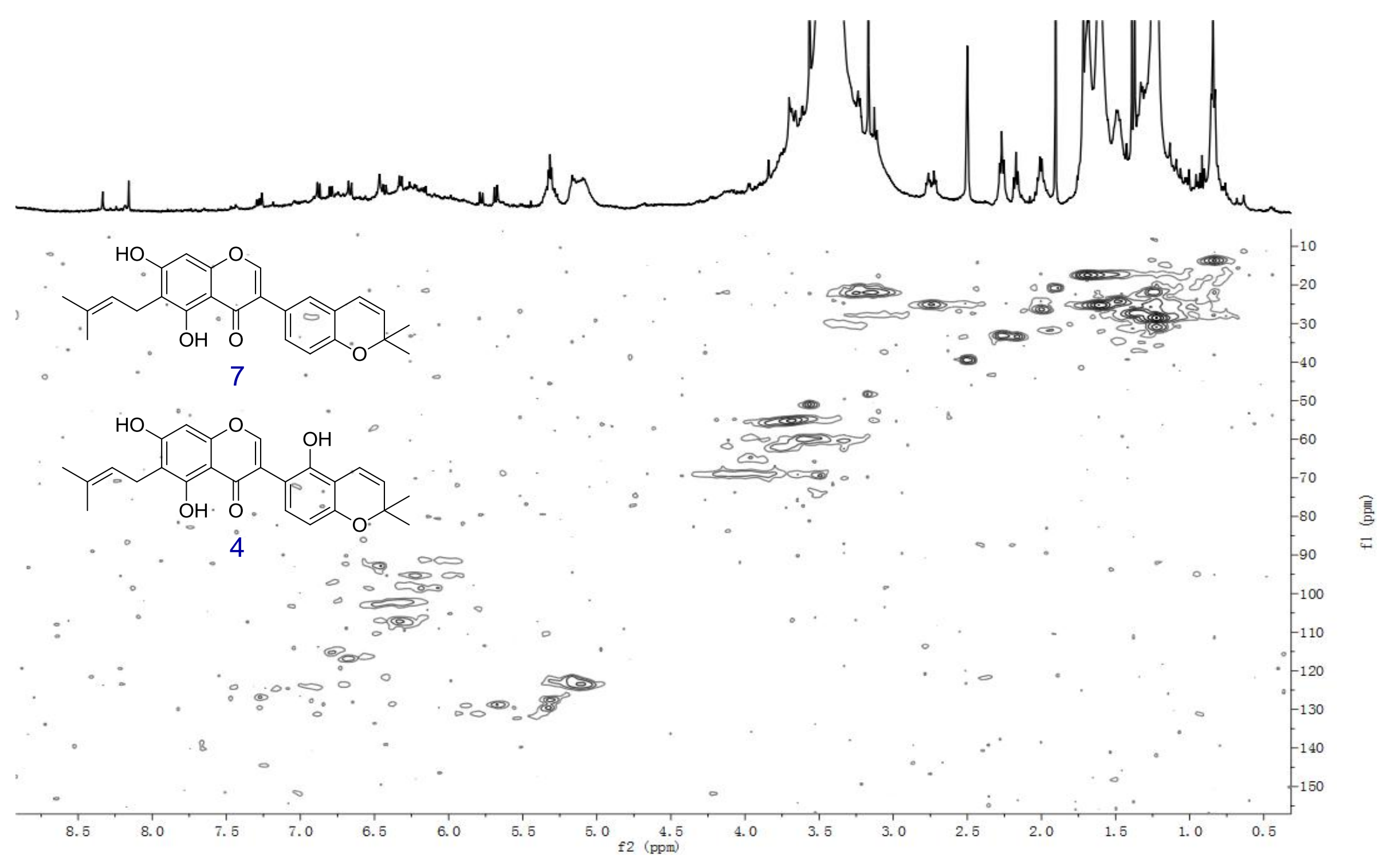

Figure S95. The HSQC spectrum of GU-MF-20 in DMSO- $d_{6}$. 


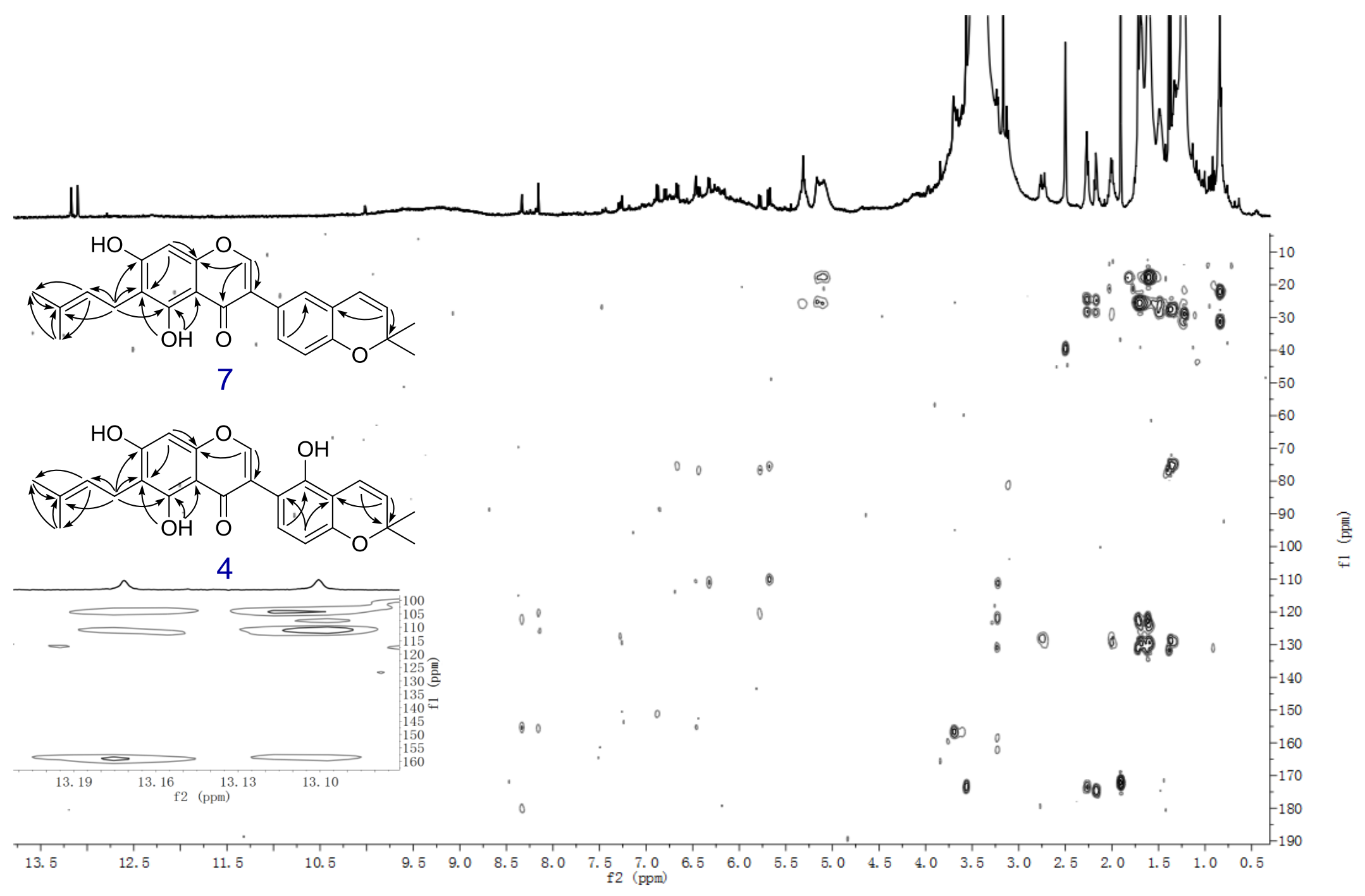

Figure S96. The HMBC spectrum of GU-MF-20 in DMSO- $d_{6}$. 

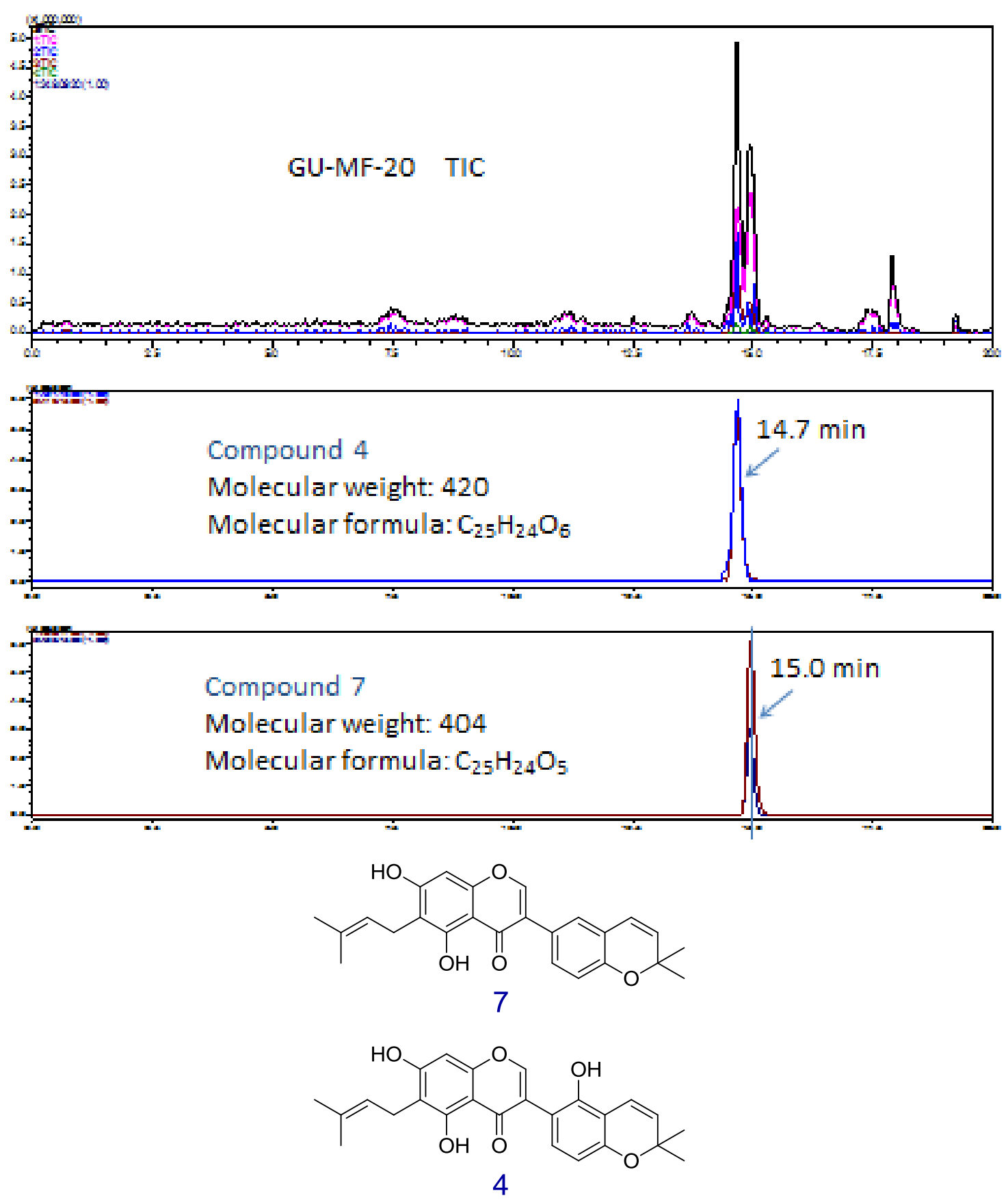

Figure S97. The IT-TOF TIC and mass chromatogram of GU-MF-20. 

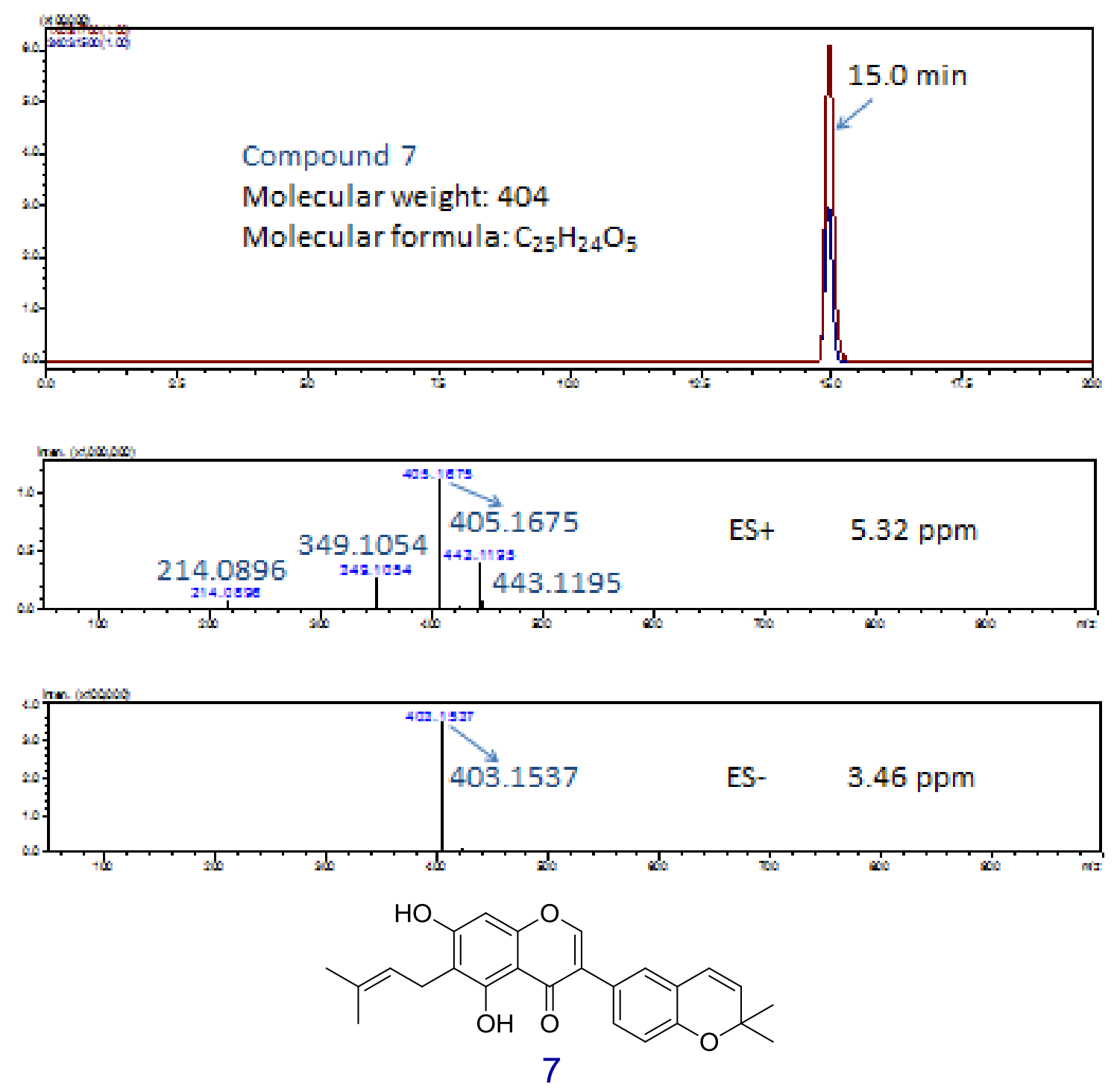

Figure S98. The (+)-HRESIMS and (-)-HRESIMS spectra of compound 7 in GU-MF-20 with extracted ions (positive and negative) for $m / z, 405$ and 403, respectively. 

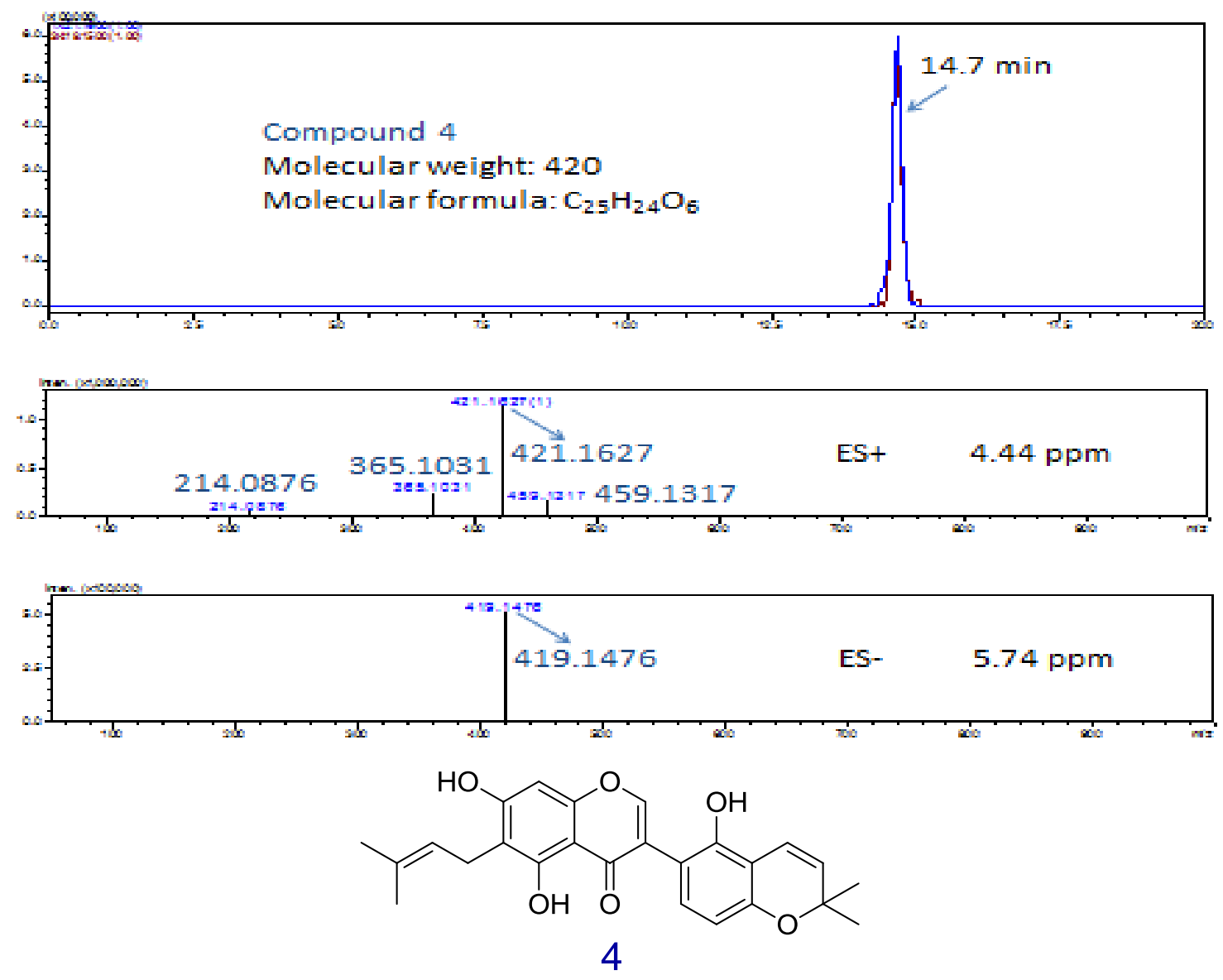

Figure S99. The (+)-HRESIMS and (-)-HRESIMS spectra of compound 4 in GU-MF-20 with extracted ions (positive and negative) for $m / z 421$ and 419, respectively. 


\section{Section 7. Flowchart of Literature Mining}

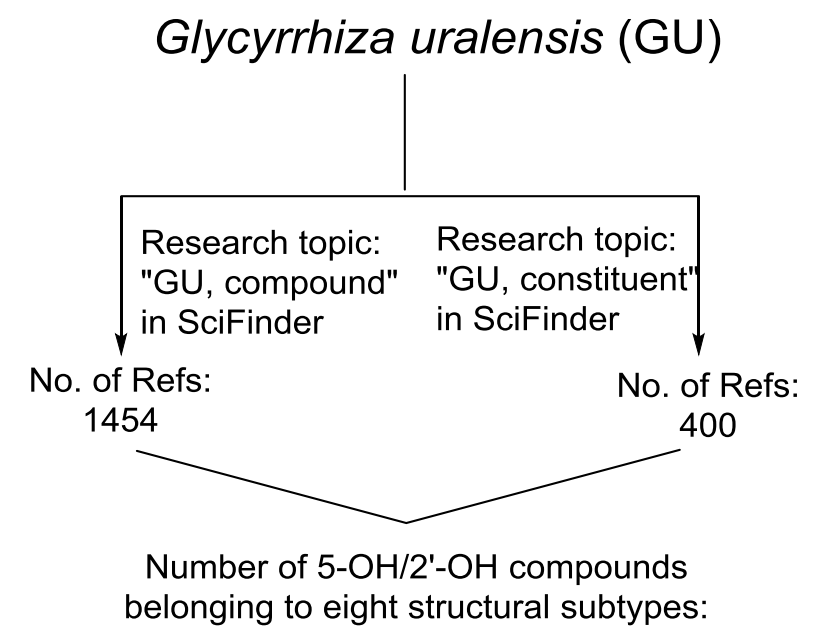

59

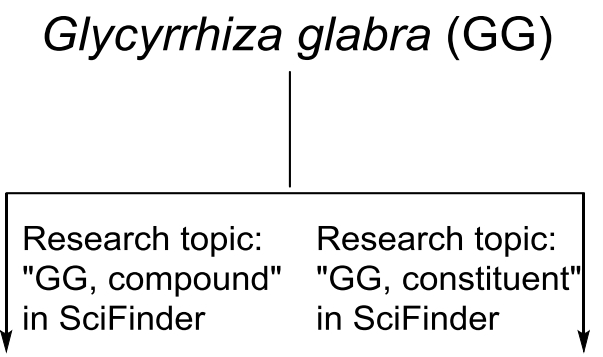

No. of Refs:

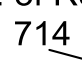

Number of $5-\mathrm{OH} / 2$ '-OH compounds belonging to eight structural subtypes:

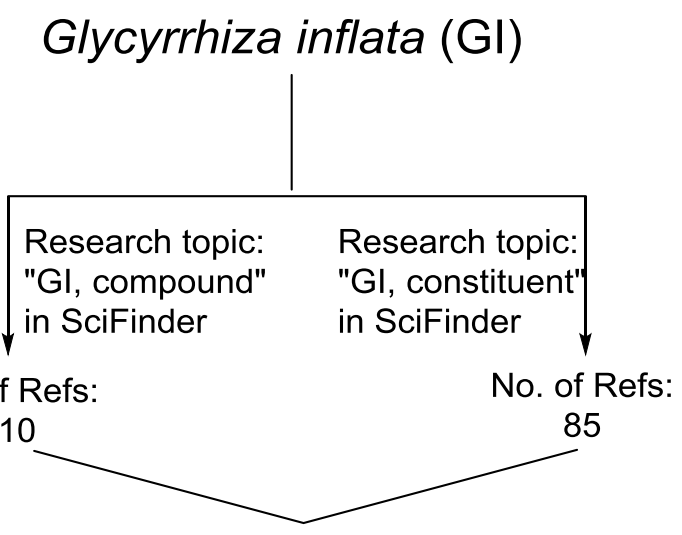

Number of $5-\mathrm{OH} / 2$ '-OH compounds belonging to eight structural subtypes:

30

Total number of 5-OH/2'-OH compounds belonging to eight structural subtypes in GU, GG, and GI (after dereplication):

SciFinder literatures were analyzed by the ended up on Apr 5, 2018.

Two criteria of data mining:

(i) compounds or constituents reported for "licorice"

(ii) structures having 5-OH flavonoids/2'-OH chalcones belonging to eight structural subtypes in "licorice" 
Section 8. SciFinder Literature Mining Results

\begin{tabular}{|c|c|c|c|c|c|c|c|}
\hline $\begin{array}{l}\text { No. of } \\
\text { Compounds } \\
\end{array}$ & Compound Name & $\begin{array}{l}\text { Hits with: } \\
\text { compound name }\end{array}$ & $\begin{array}{r}\text { Hits with: } \\
\text { cpd name + isolated }\end{array}$ & CAS No. & $\begin{array}{r}\text { Hits with: } \\
\text { CAS No. }\end{array}$ & $\begin{array}{r}\text { Hits with keywords: } \\
\text { CAS No. + isolated } \\
\end{array}$ & $\begin{array}{r}\text { Both of }{ }^{1} \mathrm{H} \text { and }{ }^{13} \mathrm{C} \text { NMR data } \\
\text { reported in DMSO- } d_{6} \text { ? }\end{array}$ \\
\hline 1 & sophoraflavanone B & 399 & 85 & $53846-50-7$ & 391 & 78 & Yes \\
\hline 2 & pinocembrin & 1900 & 648 & $480-39-7$ & 1492 & 453 & Yes \\
\hline 3 & sigmoidin B & 47 & 25 & $87746-47-2$ & 41 & 22 & Yes \\
\hline 4 & naringenin & 10722 & 1916 & $480-41-1$ & 7968 & 1199 & Yes \\
\hline 5 & licoflavanone & 63 & 24 & $119240-82-3$ & 54 & 15 & Yes \\
\hline 6 & licoisoflavanone & 27 & 9 & $66067-26-3$ & 23 & 8 & Yes \\
\hline 7 & 6-prenylnaringenin & 208 & 54 & $68236-13-5$ & 169 & 42 & Yes \\
\hline 8 & 2'-hydroxyisolupalbigenin & 7 & 6 & $121747-94-2$ & 6 & 5 & Yes \\
\hline 9 & licoflavone C & 94 & 45 & $72357-31-4$ & 83 & 37 & Yes \\
\hline 10 & lupiwighteone & 80 & 41 & $104691-86-3$ & 73 & 36 & Yes \\
\hline 11 & isolupalbigenin & 32 & 28 & $162616-70-8$ & 23 & 19 & Yes \\
\hline 12 & 2,3-dehydrokievitone & 31 & 14 & $74161-25-4$ & 30 & 13 & Yes \\
\hline 13 & licoisoflavone C & 1 & 0 & $91433-18-0$ & 1 & 0 & Yes \\
\hline 14 & gancaonin L & 20 & 5 & $129145-50-2$ & 17 & 4 & Yes \\
\hline 15 & licoisoflavone B & 78 & 35 & $66056-30-2$ & 69 & 28 & Yes \\
\hline 16 & licoisoflavone A & 74 & 27 & $66056-19-7$ & 88 & 28 & Yes \\
\hline 17 & abiochanin A & 2909 & 546 & $491-80-5$ & 2652 & 382 & Yes \\
\hline 18 & isoderrone & 28 & 24 & $121747-89-5$ & 23 & 20 & Yes \\
\hline 19 & semilicoisoflavone B & 43 & 19 & $129280-33-7$ & 38 & 16 & Yes \\
\hline 20 & apigenin & 18289 & 4987 & $520-36-5$ & 12894 & 2953 & Yes \\
\hline 21 & genistein & 30858 & 3586 & $446-72-0$ & 20846 & 2278 & Yes \\
\hline 22 & 3'-isoprenylgenistein & 5 & 5 & $68436-47-5$ & 40 & 22 & Yes \\
\hline 23 & chrysoeriol & 1742 & 868 & 491-71-4 & 1166 & 525 & Yes \\
\hline 24 & pratensein & 275 & 98 & $2284-31-3$ & 223 & 78 & Yes \\
\hline 25 & luteolin & 18213 & 5047 & $491-70-3$ & 12639 & 2917 & Yes \\
\hline 26 & epimedokoreanin D & 8 & 3 & $139163-17-0$ & 8 & 3 & Yes \\
\hline 27 & allolicoisoflavone B & 26 & 13 & $117204-81-6$ & 25 & 12 & Yes \\
\hline 28 & glycyrrhisoflavone & 52 & 22 & $116709-70-7$ & 43 & 15 & Yes \\
\hline 29 & angustone $B$ & 12 & 6 & $100462-54-2$ & 12 & 6 & Yes \\
\hline 30 & angustone $\mathrm{A}$ & 13 & 7 & $90686-13-8$ & 31 & 12 & Yes \\
\hline 31 & gancaonin A & 23 & 7 & $27762-99-8$ & 29 & 7 & Yes \\
\hline 32 & gancaonin B & 20 & 6 & $124596-86-7$ & 20 & 6 & Yes \\
\hline 33 & gancaonin $\mathrm{O}$ & 14 & 3 & $129145-53-5$ & 14 & 3 & Yes \\
\hline 34 & gancaonin $\mathrm{H}$ & 17 & 6 & $126716-35-6$ & 17 & 6 & Yes \\
\hline 35 & wighteone & 184 & 110 & $51225-30-0$ & 153 & 88 & Yes \\
\hline 36 & 6-C-prenylorobol & 11 & 7 & $66777-71-7$ & 10 & 6 & Yes \\
\hline 37 & 2',4'-dihydroxy-chalcone & 24 & 0 & 25515-43-9 & 113 & 26 & Yes \\
\hline 38 & isoliquiritigenin & 2108 & 557 & $961-29-5$ & 1762 & 442 & Yes \\
\hline 39 & licoagrochalcone A & 21 & 14 & 202815-28-9 & 27 & 13 & Yes \\
\hline 40 & homobutein & 50 & 11 & $34000-39-0$ & 46 & 10 & Yes \\
\hline 41 & isobavachalcone & 324 & 134 & $20784-50-3$ & 273 & 109 & Yes \\
\hline 42 & glabranin & 90 & 42 & 41983-91-9 & 70 & 26 & No \\
\hline 43 & 8-prenyleriodictyol & 25 & 16 & 80931-11-9 & 23 & 14 & No \\
\hline 44 & gancaonin E & 22 & 3 & $124596-89-0$ & 22 & 3 & No \\
\hline 45 & euchestraflavanone A & 3 & 2 & $80510-05-0$ & 42 & 21 & No \\
\hline 46 & 3'-( $\gamma, \gamma$-dimethylallyl)-kievitone & 5 & 2 & $141846-47-1$ & 7 & 3 & No \\
\hline 47 & dihydrolicoisoflavone & 4 & 4 & $164163-92-2$ & 3 & 3 & No \\
\hline 48 & $\begin{array}{l}\text { (2S)-3'-(2-hydroxy-3-methylbut-3- } \\
\text { enyl)-4',5,7-trihydroxy- } \\
\text { dihydroflavanone }\end{array}$ & 1 & 1 & none & 0 & 0 & No \\
\hline 49 & glyasperin $\mathrm{F}$ & 20 & 13 & $145382-61-2$ & 16 & 10 & No \\
\hline 50 & isoglabranin & 42 & 24 & $55051-77-9$ & 41 & 23 & No \\
\hline 51 & 6-prenyleriodictyol & 10 & 8 & $80931-12-0$ & 19 & 11 & No \\
\hline 52 & paratocarpin $\mathrm{L}$ & 14 & 6 & $97134-79-7$ & 14 & 6 & No \\
\hline 53 & glisoflavanone & 17 & 11 & $314734-02-6$ & 14 & 8 & No \\
\hline 54 & gancaonin M & 15 & 5 & $129145-51-3$ & 15 & 5 & No \\
\hline 55 & vogelin C & 8 & 6 & $423163-93-3$ & 5 & 4 & No \\
\hline 56 & glyurallin B & 18 & 7 & $199331-53-8$ & 17 & 6 & No \\
\hline 57 & orobol & 338 & 132 & $480-23-9$ & 264 & 94 & No \\
\hline 58 & allolicoisoflavone A & 5 & 2 & $82448-67-7$ & 7 & 2 & No \\
\hline 59 & gancaonin Q & 17 & 10 & $134958-52-4$ & 13 & 7 & No \\
\hline 60 & 6,5'-diprenylluteolin & 1 & 1 & $782503-66-6$ & 2 & 1 & No \\
\hline 61 & luteone & 108 & 35 & $41743-56-0$ & 86 & 28 & No \\
\hline 62 & gancaonin $\mathrm{N}$ & 7 & 4 & $129145-52-4$ & 7 & 4 & No \\
\hline 63 & isoangustone $\mathrm{A}$ & 48 & 24 & $129280-34-8$ & 37 & 15 & No \\
\hline 64 & isochandalone & 10 & 9 & $121747-90-8$ & 9 & 8 & No \\
\hline 65 & 5-prenylbutein & 9 & 5 & $813463-19-3$ & 7 & 3 & No \\
\hline 66 & kanzonol B & 19 & 11 & $155233-19-5$ & 17 & 10 & No \\
\hline 67 & licochalcone $\mathrm{G}$ & 8 & 2 & $1261240-29-2$ & 6 & 1 & No \\
\hline 68 & isoliquiritin & 564 & 103 & $5041-81-6$ & 444 & 70 & No \\
\hline 69 & isoliquiritin apioside & 171 & 36 & $120926-46-7$ & 146 & 28 & No \\
\hline 70 & 6"-0-acetylisoliquiritin & 4 & 2 & 1640131-17-4 & 1 & 1 & No \\
\hline 71 & $\begin{array}{l}\text { 1-(2,4-dihydroxyphenyl)-3-[4- } \\
\text { methoxy-3-(3-methylbut-2-enyl)- } \\
\text { phenyl]prop-2-en-1-one }\end{array}$ & 5 & 0 & $943860-39-7$ & 5 & 0 & No \\
\hline 72 & morachalcone A & 51 & 41 & $76472-88-3$ & 48 & 32 & No \\
\hline 73 & corylifol B & 16 & 8 & 775351-90-1 & 16 & 8 & No \\
\hline 74 & kanzonol C & 39 & 20 & $151135-82-9$ & 36 & 17 & No \\
\hline 75 & $\begin{array}{l}\text { 2,3',4,4'-tetrahydroxy-3,5'- } \\
\text { diprenylchalcone }\end{array}$ & 0 & 0 & $938190-29-5$ & 2 & 2 & No \\
\hline 76 & $\begin{array}{l}\text { 6",6"-dimethylpyrano }[2 ", 3 ": 4,5]- \\
3^{\prime}-\gamma, y \text {-dimethylally-2',3, 4'- } \\
\text { trihydroxychalcone }\end{array}$ & 0 & 0 & $151135-83-0$ & 11 & 8 & No \\
\hline
\end{tabular}

Note: The literature mining was achieved up to Apr 5, 2018 


\section{Section 9. Spreadsheet*}

*Raw NMR data (FIDs) and qNMR calculation spreadsheets are available at DOI: 10.7910/DVN/NFQNJQ.

\section{Determination of residual hydrogen in deuterated NMR solvent*}

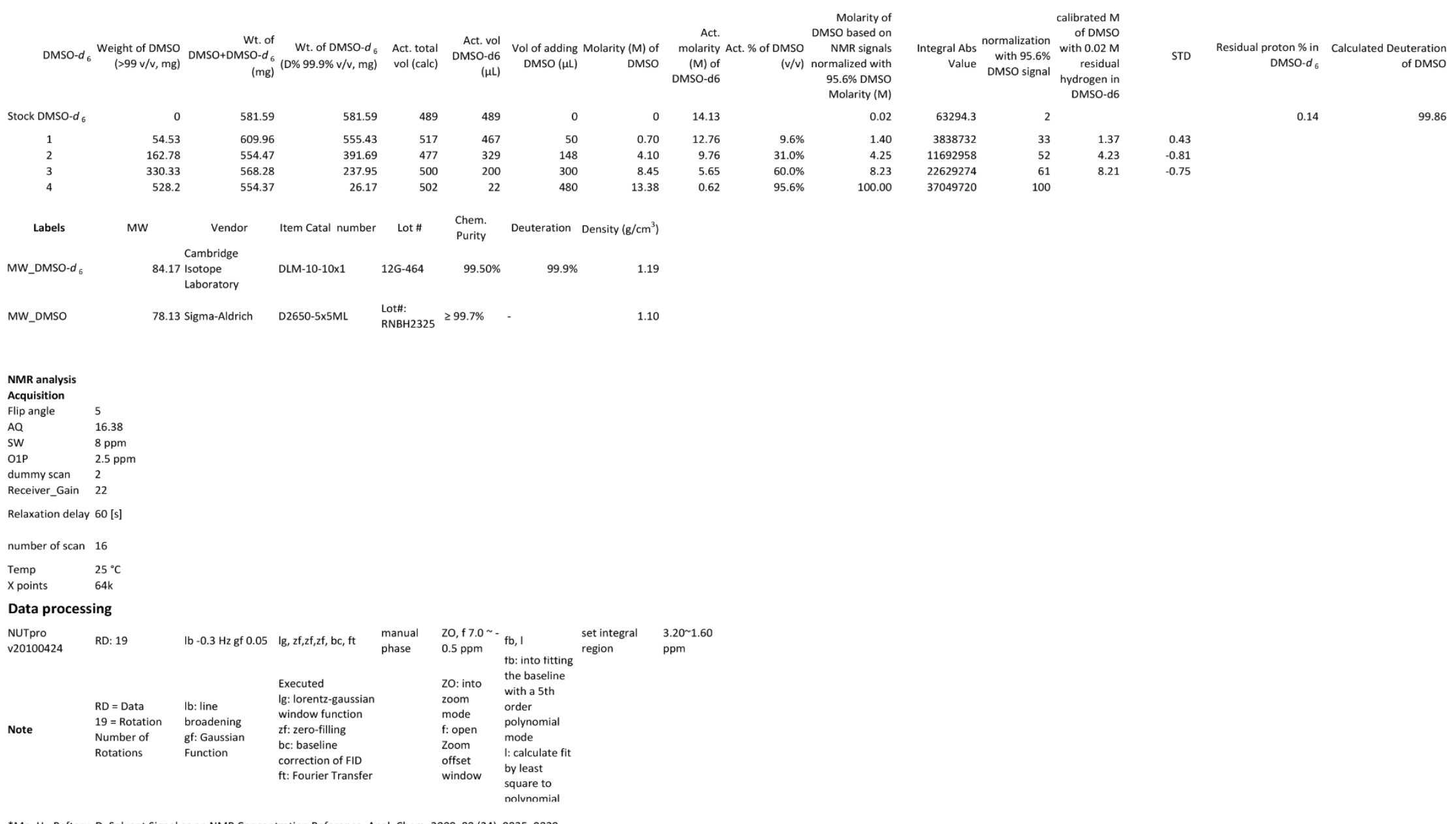

"Mo, H.; Raftery, D. Solvent Signal as an NMR Concentration Reference, Anal. Chem. 2008, 80 (24), 9835-9839. 


\section{Section 10. Absolution Quantitation}

*Raw NMR data (FIDs) and qNMR calculation spreadsheets are available at DOI: 10.7910/DVN/NFQNJQ.

\begin{tabular}{|c|c|c|c|c|c|c|c|c|}
\hline $\begin{array}{l}\text { NMR Tube } \\
5 \mathrm{~mm}\end{array}$ & $\begin{array}{l}\text { Solvent } \\
\text { Volume }\end{array}$ & $\begin{array}{l}\text { Total integral of } \\
\text { residual DMSO- } d_{5}\end{array}$ & \multicolumn{5}{|c|}{ Quantity of 15 identified metabolites } & \multirow[t]{2}{*}{ Identified compounds } \\
\hline $\begin{array}{l}\text { Absolute } \\
\text { Integral }\end{array}$ & $\begin{array}{l}\text { Chemical } \\
\text { shift }\end{array}$ & $\begin{array}{l}\text { Integral (relative to } \\
\text { residual DMSO- } d_{5} \text { ) }\end{array}$ & $\begin{array}{l}\text { Identified } \\
\text { Compound } \\
\text { No. }\end{array}$ & M.W. & $\begin{array}{l}\text { Position in total } \\
\text { quant. Metab. }\end{array}$ & $\begin{array}{c}\mu \mathrm{M} \\
\left(5-\mathrm{OH} / 2^{\prime}-\mathrm{OH}\right)\end{array}$ & $\begin{array}{c}\text { Amount } \\
\text { in GU-MF } \\
(\mu \mathrm{g})\end{array}$ & \\
\hline $30,637,212$ & 13.538 & 2102.605063 & & & 158 & \multicolumn{2}{|c|}{199.75 n/a } & tentatively identified as 4'-O-glucoside chalcone \\
\hline $25,602,020$ & 12.177 & 1757.04424 & & & 157 & \multicolumn{2}{|c|}{$166.92 \mathrm{n} / \mathrm{a}$} & not belong to any classified groups \\
\hline $25,156,598$ & 12.861 & 1726.475318 & 12 & 352.34 & 156 & 164.02 & 34.7 & licoisoflavone B \\
\hline $24,633,712$ & 13.275 & 1690.590109 & 5 & 422.48 & 155 & 160.61 & 40.7 & isoangustone $A$ \\
\hline $23,820,336$ & 12.955 & 1634.768825 & 14 & 352.34 & 154 & 155.30 & 32.8 & semilicoisoflavone B \\
\hline $21,554,500$ & 12.288 & 1479.266482 & 3 & 354.36 & 153 & 140.53 & 29.9 & licoisoflavanone \\
\hline $17,429,514$ & 12.269 & 1196.172301 & 2 & 424.50 & 152 & 113.64 & 28.9 & 3'-( $\gamma, \gamma$-dimethylallyl)-kievitone \\
\hline $13,168,966$ & 12.864 & 903.7746184 & 11 & 354.36 & 149 & 85.86 & 18.3 & licoisoflavone A \\
\hline $12,065,097$ & 13.008 & 828.0170544 & 13 & 354.36 & 148 & 78.66 & 16.7 & glycyrrhisoflavone \\
\hline $7,746,451$ & 12.915 & 531.6321567 & 15 & 336.34 & 144 & 50.51 & 10.2 & isoderrone \\
\hline $5,132,023$ & 12.567 & 352.2062498 & 1 & 422.48 & 140 & 33.46 & 8.5 & 6-prenyl-licoisoflavanone \\
\hline $4,028,154$ & 13.214 & 276.4486858 & 6 & 420.46 & 136 & 26.26 & 6.6 & gancaonin $\mathrm{H}$ \\
\hline $3,892,592$ & 13.231 & 267.1451843 & 8 & 338.36 & 135 & 25.38 & 5.2 & wighteone \\
\hline $3,524,635$ & 13.186 & 241.8926172 & 7 & 404.46 & 130 & 22.98 & 5.6 & isochandalone \\
\hline $3,292,242$ & 12.908 & 225.9436889 & 10 & 338.36 & 129 & 21.46 & 4.4 & lupiwighteone \\
\hline $2,440,132$ & 13.222 & 167.4641249 & 9 & 406.48 & 122 & 15.91 & 3.9 & 6,8-diprenylgenistein \\
\hline \multirow[t]{5}{*}{$2,246,471$} & 13.117 & 154.1733399 & 4 & 420.46 & 118 & 14.65 & 3.7 & angustone $\mathrm{B}$ \\
\hline & & & Average & 380.14 & & 73.95 & 16.7 & \\
\hline & & & Sum & & & 1109.23 & 250.0 & \\
\hline & & Isolates average wt. ir & n NMR tube & & & & 16.9 & \\
\hline & Total per & rcentage of isolates in & flavonome & & & 38.52 & & \\
\hline
\end{tabular}

$\mathrm{n} / \mathrm{a}$ : Not be detected by NMR in enriched HPLC fractions 